\title{
Characteristics of turbulence and design of solid removal system in the quiescent zone of an aquaculture raceway
}

\author{
Eric G. Fizer \\ West Virginia University
}

Follow this and additional works at: https://researchrepository.wvu.edu/etd

\section{Recommended Citation}

Fizer, Eric G., "Characteristics of turbulence and design of solid removal system in the quiescent zone of an aquaculture raceway" (2009). Graduate Theses, Dissertations, and Problem Reports. 4463.

https://researchrepository.wvu.edu/etd/4463

This Thesis is protected by copyright and/or related rights. It has been brought to you by the The Research Repository @ WVU with permission from the rights-holder(s). You are free to use this Thesis in any way that is permitted by the copyright and related rights legislation that applies to your use. For other uses you must obtain permission from the rights-holder(s) directly, unless additional rights are indicated by a Creative Commons license in the record and/ or on the work itself. This Thesis has been accepted for inclusion in WVU Graduate Theses, Dissertations, and Problem Reports collection by an authorized administrator of The Research Repository @ WVU. For more information, please contact researchrepository@mail.wvu.edu. 


\title{
Characteristics of Turbulence and Design of Solid Removal System in the Quiescent Zone of an Aquaculture Raceway
}

\author{
Eric G. Fizer \\ Thesis submitted to the \\ College of Engineering and Mineral Resources \\ at West Virginia University \\ in partial fulfillment of the requirements \\ for the degree of: \\ Master of Science \\ in \\ Mechanical Engineering
}

Donald D. Gray, Ph.D., Chair

John M. Kuhlman, Ph.D.

Gary J. Morris, Ph.D.

Wade W. Huebsch, Ph.D.

Kenneth Semmens, Ph.D.

Department of Mechanical and Aerospace Engineering

Morgantown, West Virginia

2009

Keywords: Turbulence Measurements, Aquaculture Raceway, Quiescent Zone, Dogwood Lake, Acoustic Doppler Velocimeter 


\title{
ABSTRACT \\ Characteristics of Turbulence and Design of Solid Removal System in the Quiescent Zone of an Aquaculture Raceway
}

\author{
Eric G. Fizer
}

An aquaculture raceway is a water carrying, open channel with a rectangular crosssection used to raise fish. The first objective of this research is to measure the flow characteristics within a small section of an aquaculture raceway system called the quiescent zone, and determine if the flow through the quiescent zone contains two-dimensional, fully developed, turbulent open channel flow characteristics. If any discrepancies exist, the cause or causes of the discrepancies are to be analyzed. The measured flow characteristics are intended to improve previous measurements taken in the quiescent zone of a rectangular aquaculture raceway system. The second objective is to design an efficient non-labor intensive system for removing solid particles from the bottom of a quiescent zone. The flow through the raceway at Dogwood Lake, West Virginia, contains subcritical flow and has a Reynolds number of approximately $2 \times 10^{4}$, which indicates that the flow is mildly turbulent.

The first objective is accomplished by using an Acoustic Doppler Velocimeter (ADV) to measure velocities in three dimensions at a point. The velocities measured by the ADV are sampled at $10 \mathrm{~Hz}$ and averaged over a 360 second time interval in order to calculate a mean velocity and other statistical parameters in each direction. ADV measurements allow for flow characteristics such as mean velocity profiles, turbulence intensity (TI) profiles, and Reynolds stress profiles to be measured within the quiescent zone. The mean velocity, turbulence intensity, and Reynolds stress profiles seem to contain two-dimensional, fully developed, turbulent characteristics in certain respects, but vary dramatically in others. The large eddy range, inertial subrange, and dissipation range are difficult to segregate in several measurements of the energy spectrum. Discrepancies between these measurements and two-dimensional, fully developed, turbulent open channel flow are due to disturbances and geometry limitations of the raceway. It was found that a disturbance caused by a small meshed screen separating the fish from the quiescent zone may reduce turbulence intensity values in the quiescent zone.

The second objective is accomplished by determining several possibilities for a cleaning mechanism and choosing the mechanism that best fits the constraints and limitations at Dogwood Lake. Two mechanisms were constructed and tested in one quiescent zone at Dogwood Lake. The first constructed cleaning mechanism was the sloped false bottom (SFB). The SFB is a false bottom with sloped walls used to collect solids into a concentrated area. A pre-existing standpipe system is used to remove the solids from the concentrated area. The second constructed mechanism is the siphon system (SS). The SS is powered by a siphon connected to a false bottom that evenly distributes suction throughout the false bottom. Both mechanisms failed to clean the entire bottom thoroughly. 


\section{Contents}

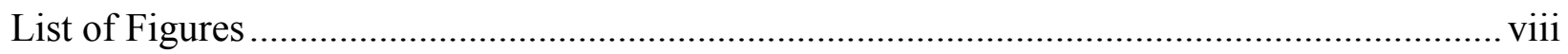

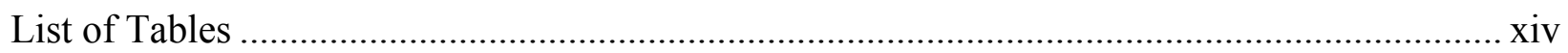

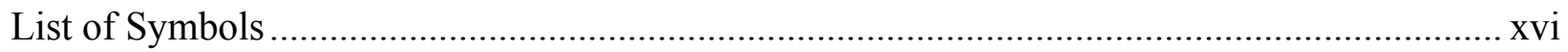

Part 1: Characteristics of Turbulence in the Quiescent Zone ........................................................ 1

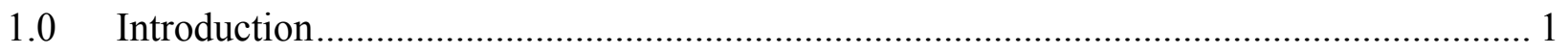

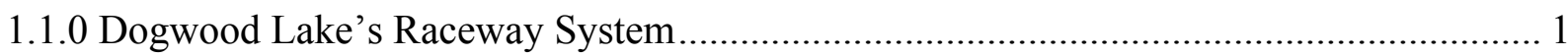

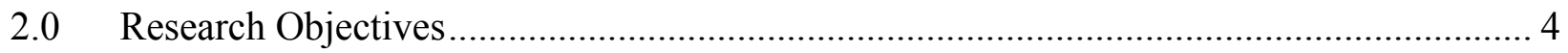

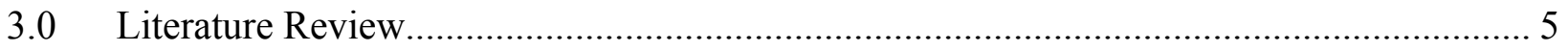

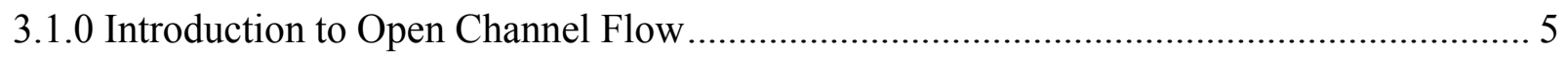

3.2.0 Introduction to Measuring Open Channel Flow........................................................... 5

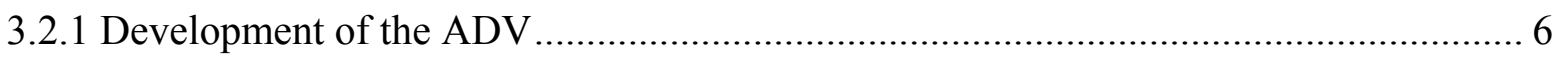

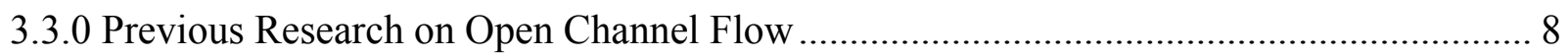

3.3.1 LDV Velocity Measurements in a 17 Meter Flume ……................................................ 8

3.3.2 Open Channel Flow Measurements with a Laser Doppler Anemometer ...................... 11

3.3.3 Turbulent Open Channel Flow Over Smooth and Rough Boundaries ........................... 11

3.3.4 ADV Measurements in Aquaculture Raceway Systems ………................................ 11

3.4.0 Previous Research on Evaluating the Capability of ADVs............................................. 13

3.4.1 Direct Measurements of Reynolds Stress with an ADV ............................................. 13

3.4.2 Evaluation of the ADV for Turbulence Measurements............................................. 14

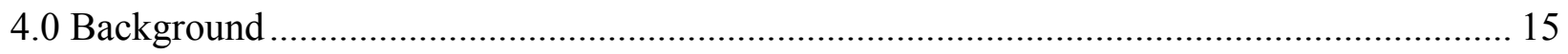

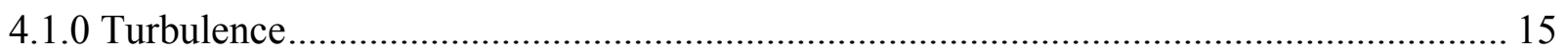

4.1.1 Calculating Theoretical/Empirical RMS .................................................................. 20

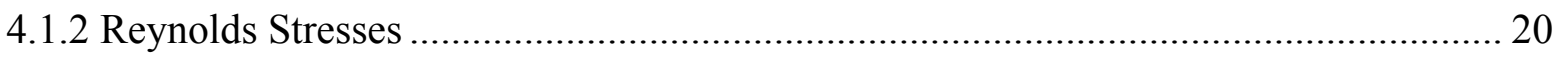

4.2.0 Two-Dimensional Fully Developed Turbulent Open Channel Flow ............................... 21 


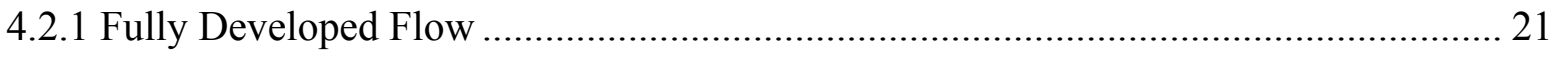

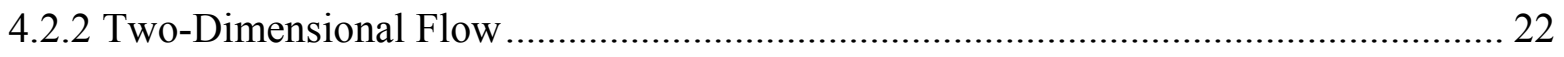

4.3.0 Dimensionless Parameters................................................................................ 22

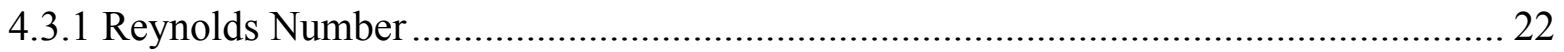

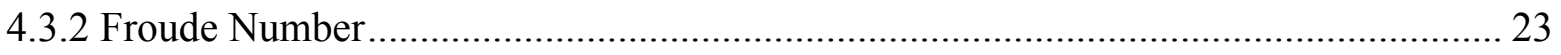

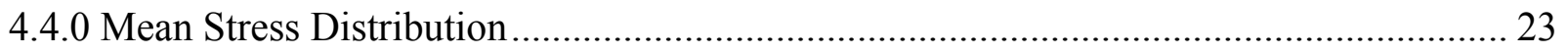

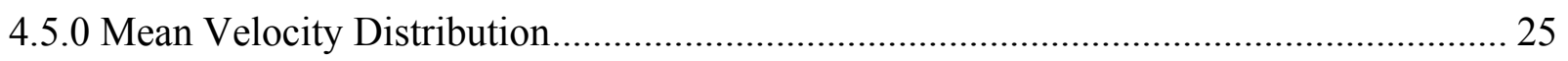

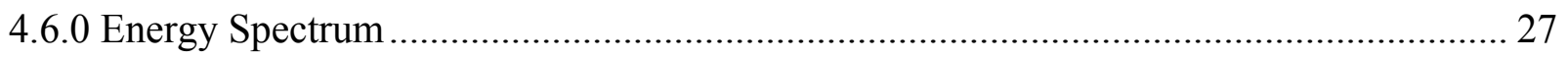

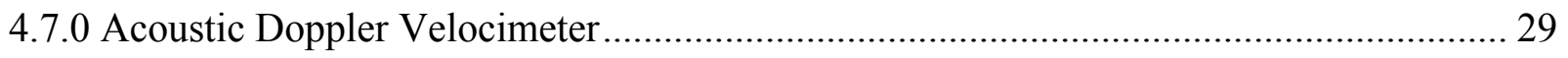

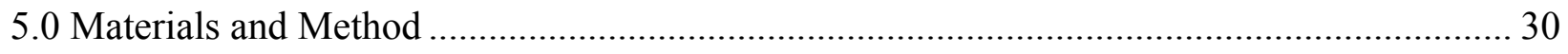

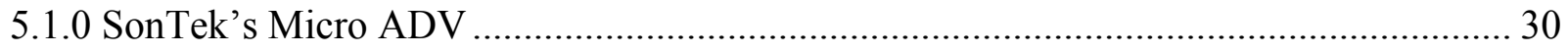

5.1.1 Receiving Accurate Measurements from the Micro ADV ..................................... 32

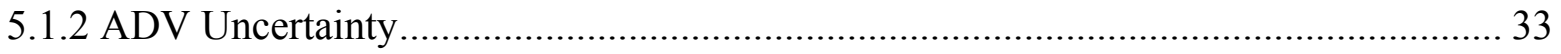

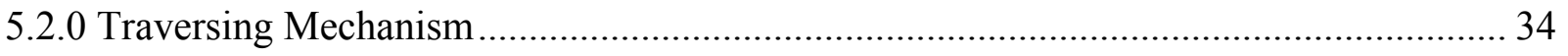

5.2.1 Traversing Mechanism Alignment Uncertainty .................................................. 38

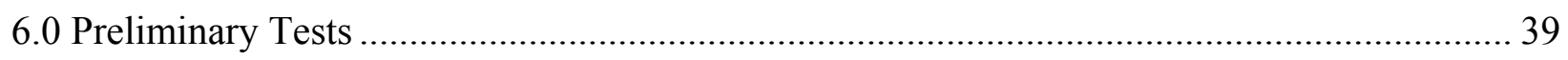

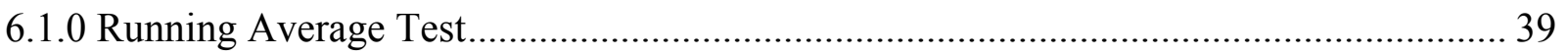

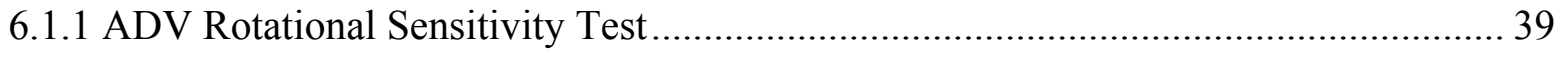

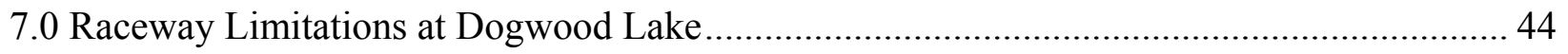

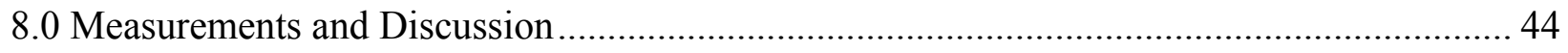

8.1.0 Description of Quiescent Zones and Collected Measurements................................... 44

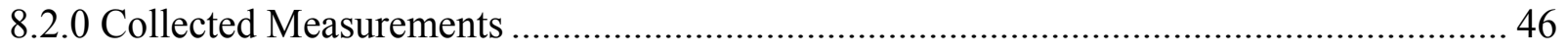

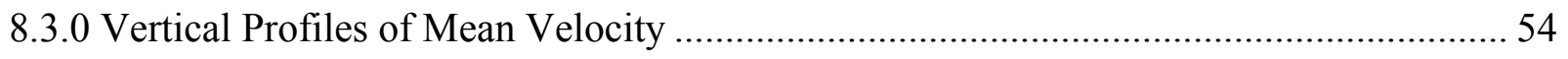

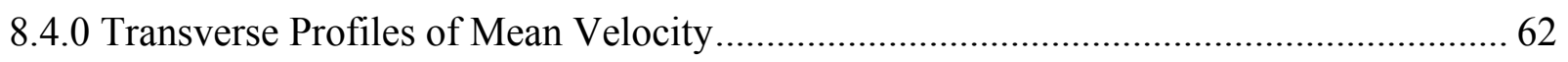

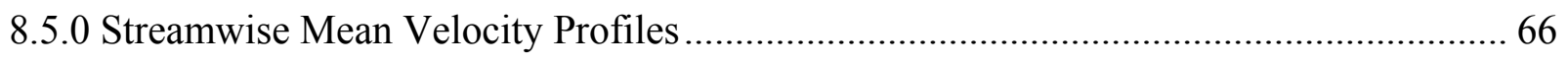


8.6.0 Vertical Profiles of Root Mean Square (RMS) Velocities ................................................ 69

8.7.0 Turbulence Intensity Profiles ....................................................................................... 73

8.8.0 Vertical Profiles of Reynolds Stresses ........................................................................ 76

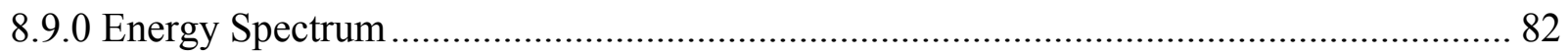

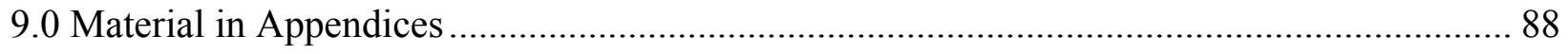

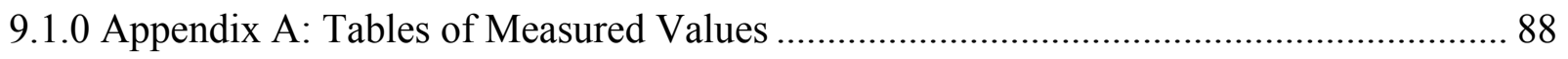

9.2.0 Appendix B: Mean Velocity Profiles with Standard Deviation ....................................... 88

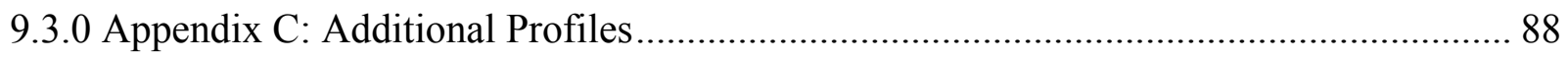

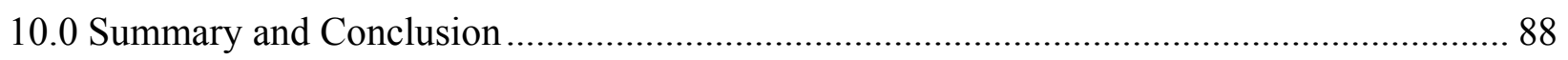

10.1.0 Disturbances in the Raceway System at Dogwood Lake ............................................... 89

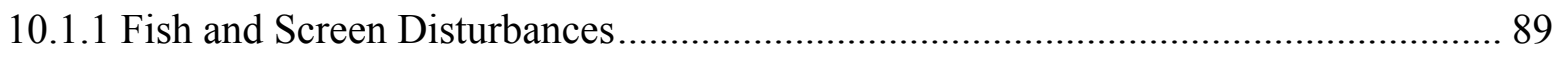

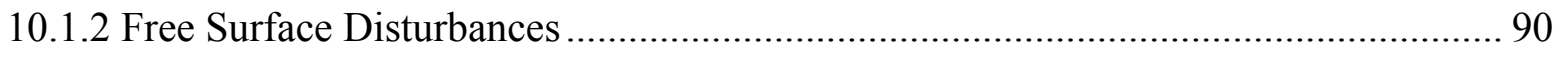

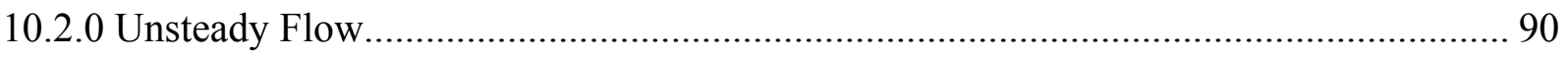

10.3.0 Low Reynolds Number ....................................................................................... 90

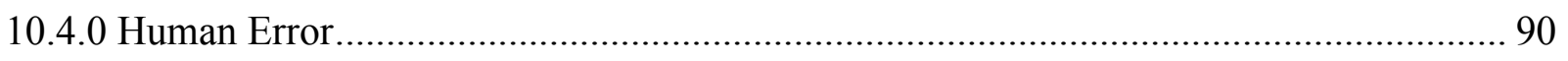

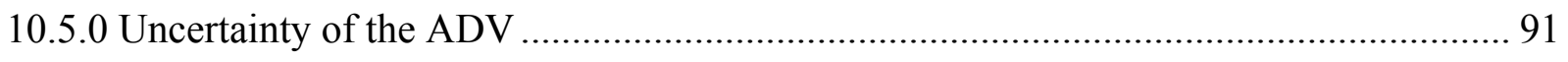

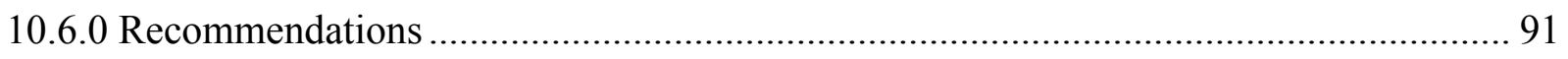

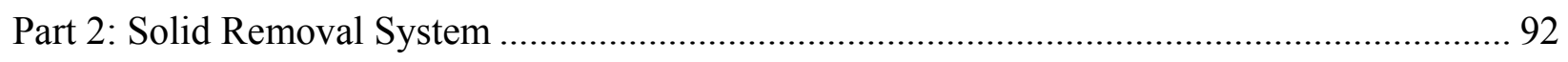

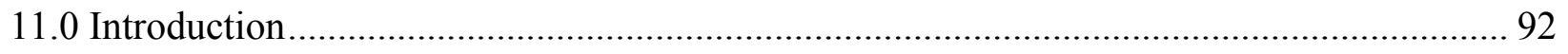

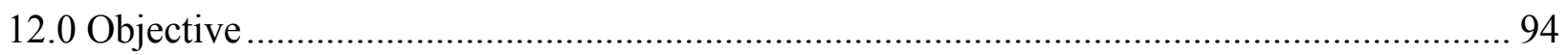

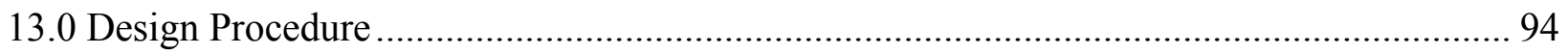

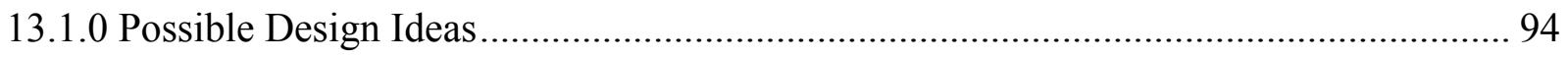

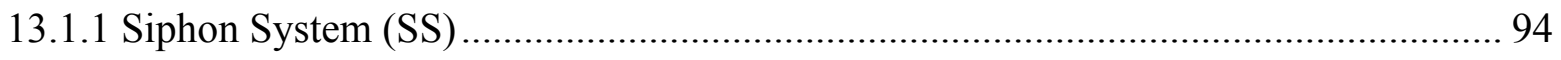

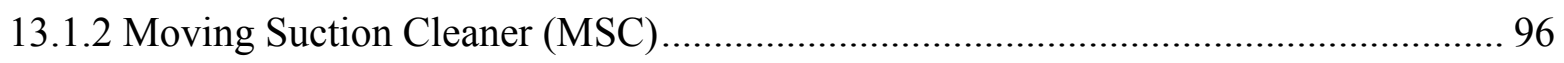

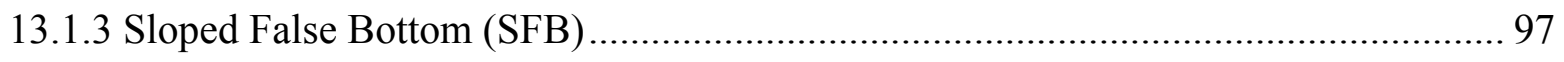




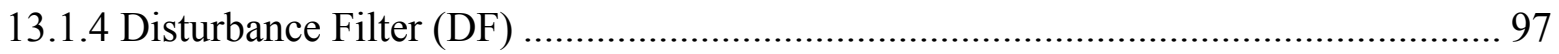

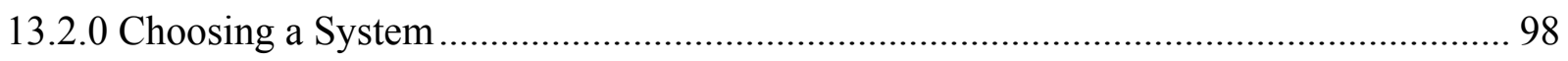

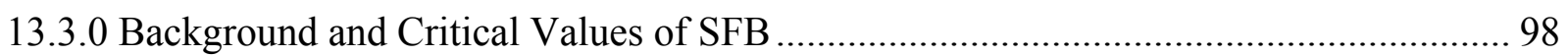

13.3.1 Determining Required Force to Move a Particle.......................................................... 99

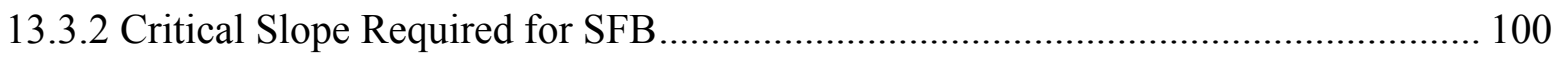

13.3.3 Critical Slope for Removing Particles with the Aid of Moving Water ................... 100

13.3.4 Critical Slope for Removing Particles with Resisting Water ................................. 102

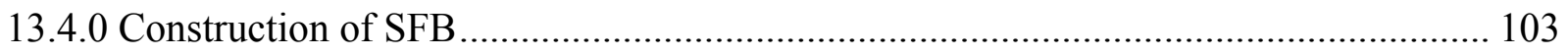

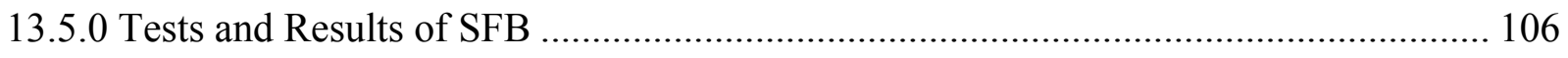

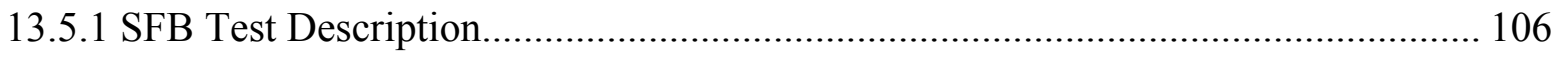

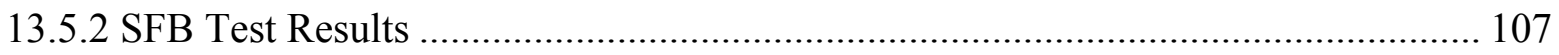

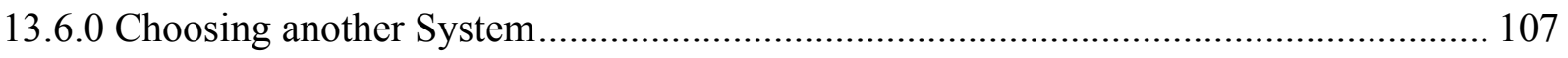

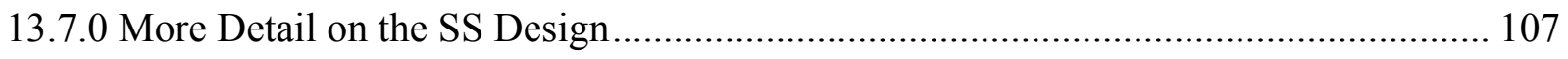

13.8.0 Background Required for Siphon System Design..................................................... 110

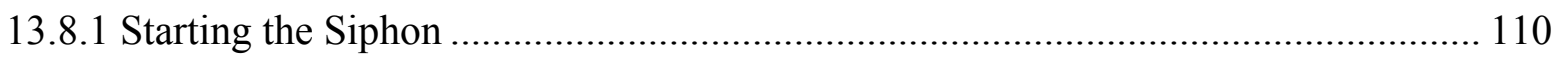

13.8.2 Minimum Required Volume of Siphon Tub........................................................... 111

13.8.3 Finding Flow Rate Required from SS to Cause Critical Shear Stress on Solids...... 111

13.8.4 Estimating Flow Rate that the Siphon System can Provide .................................... 112

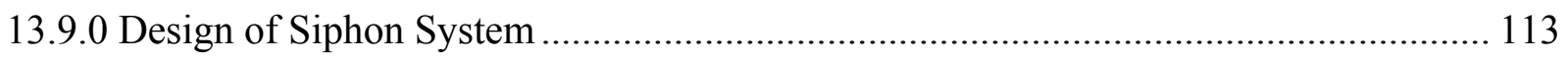

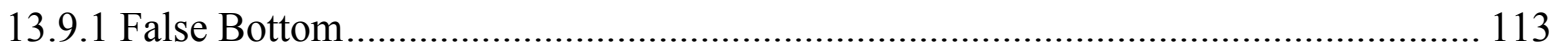

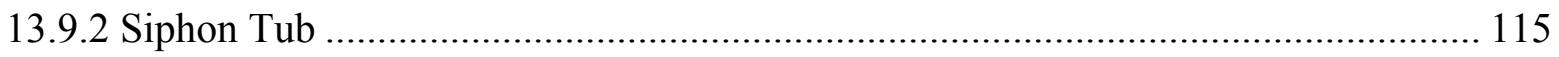

13.10.0 Construction of Siphon System.......................................................................... 115

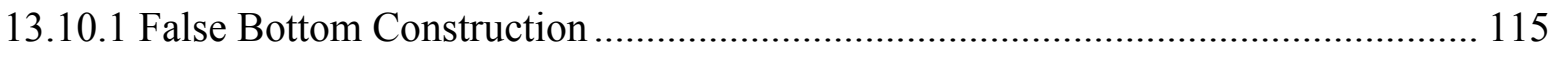

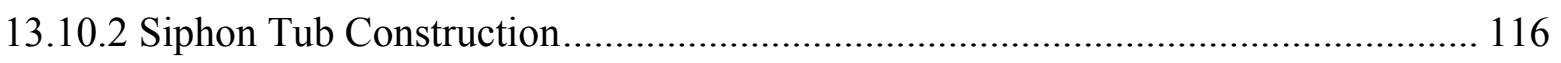

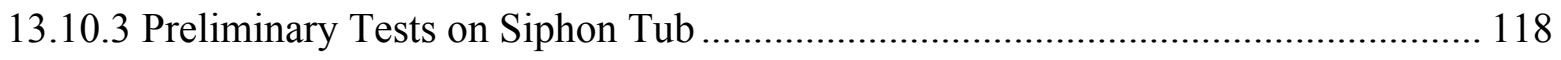




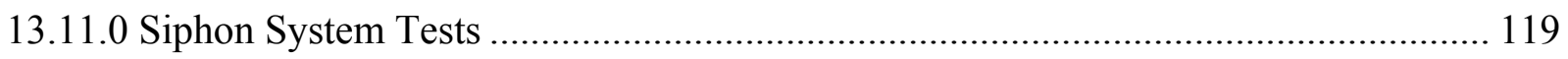

13.11.1 Siphon System Test 1 Results .............................................................................. 119

13.11.2 New False Bottom ................................................................................................ 120

13.11.3 Siphon System Test 2 Results ....................................................................... 120

14.0 Cleaning Mechanism Conclusions and Suggestions …….................................................. 123

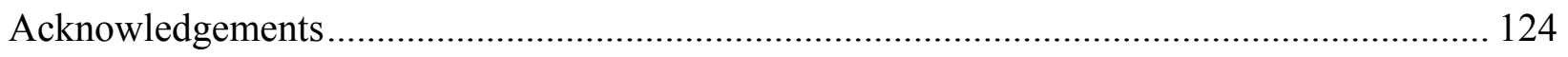

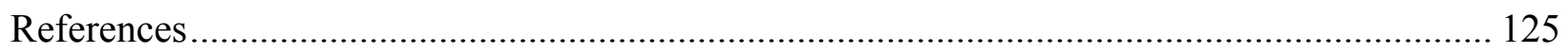

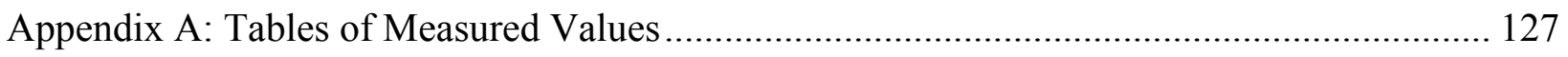

Appendix B: Mean Velocity Profiles with Standard Deviation................................................... 154

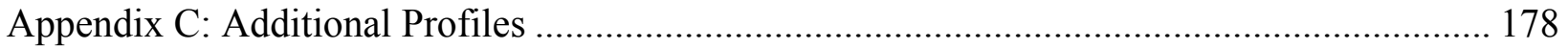




\section{List of Figures}

Figure 1: Dimensions of Raceway at Dogwood Lake ……….................................................. 2

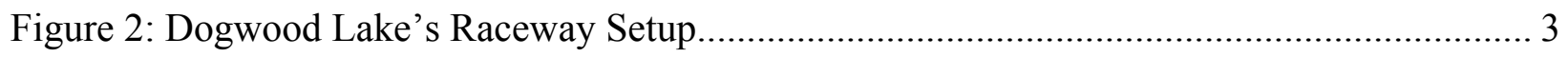

Figure 3: Downstream View of the Raceway System at Dogwood Lake..................................... 3

Figure 4: ADV Probe Tip Developed by Kraus et al. (1994) ........................................................ 7

Figure 5: Horizontal Velocity Measurement Comparison between ADV and LDV (Kraus et al.,

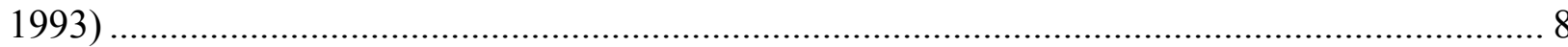

Figure 6: Dimensionless Semi-Log Vertical Profile of Mean Streamwise Velocity (Trowbridge et

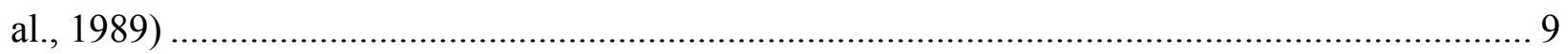

Figure 7: Vertical Profile of Reynolds Stresses (Trowbridge et al., 1989)................................. 10

Figure 8: Dimensionless Vertical Profile of RMS Vertical Velocity (Trowbridge et al., 1989).. 10

Figure 9: 3-D Plot of Resultant Velocity at Dogwood Lake (Rumberg, 2004) ............................. 12

Figure 10: Turbulent Fluctuation Comparison between ADV and LDV (Lohrmann et al, 1995) 14

Figure 11: Mean Streamwise Velocity Comparison between ADV and LDV (Voulgaris and

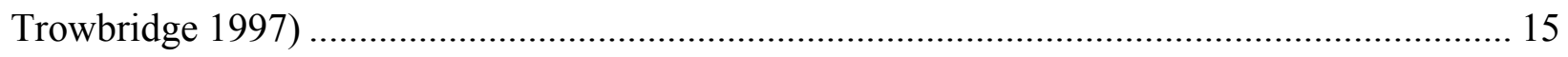

Figure 12: Running Average of Streamwise Velocity ........................................................... 17

Figure 13: Streamwise Velocity Fluctuation …………........................................................ 18

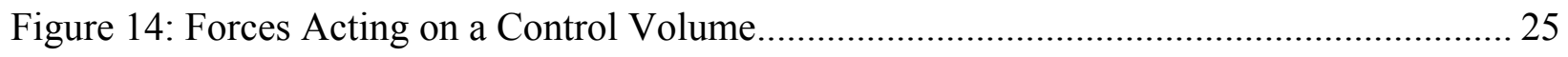

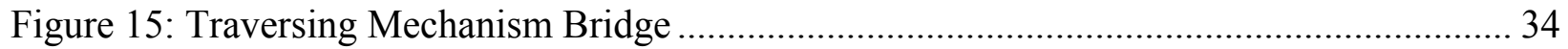

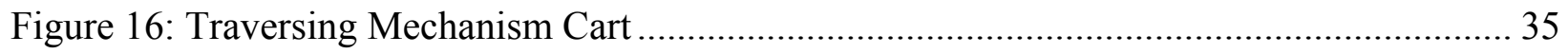

Figure 17: Traversing Mechanism Aluminum Beam …………................................................. 35

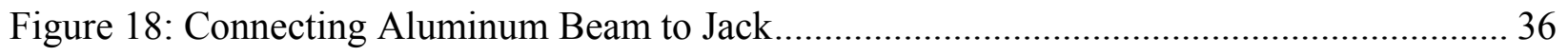

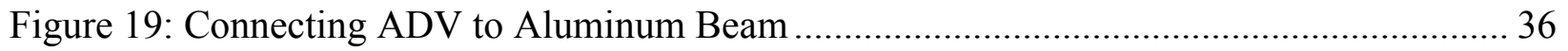

Figure 20: Complete Setup of Traversing Mechanism ............................................................ 37

Figure 21: Mean Streamwise Velocity Angular Sensitivity .......................................................... 41

Figure 22: Mean Transverse Velocity Angular Sensitivity ........................................................ 42

Figure 23: Mean Vertical Velocity Angular Sensitivity ……….................................................. 43

Figure 24: Large Screen Used to Separate Fish Zone from Quiescent Zone 1 and Corresponding

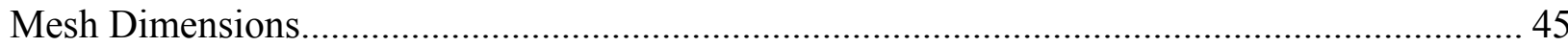

Figure 25: Small Screen Used to Separate the Fish Zone from Quiescent Zone 2 and

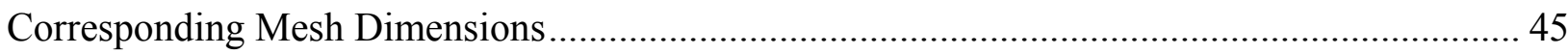


Figure 26: Location of Vertical Profile Measurements taken on August 30, 2008 ................... 47

Figure 27: Location of Vertical Profile Measurements taken on September 4, 2008................ 48

Figure 28: Location of Vertical Profile Measurements taken on September 30, 2008............... 49

Figure 29: Location of Vertical Profile Measurements taken on October 9, 2008.................... 50

Figure 30: Location of Transverse Profile Measurements taken on November 4, 2008 ............. 51

Figure 31: Location of Transverse Profile Measurements taken on November 6, 2008 ............ 52

Figure 32: Location of Streamwise Profile Measurements taken on November 26, 2008 .......... 53

Figure 33: Location of Streamwise Profile Measurements taken on November 29, 2008 .......... 54

Figure 34: Vertical Profiles of Mean Streamwise Velocity .................................................. 55

Figure 35: Huggins' Vertical Profile of Mean Streamwise Velocity (Huggins, 2003)............... 56

Figure 36: Vertical Profile of Mean Streamwise Velocity (Rumberg, 2004)............................ 57

Figure 37: Running Average all Three Velocity Components (Rumberg, 2004)...................... 58

Figure 38: Vertical Profiles of Mean Transverse Velocity .................................................... 59

Figure 39: Vertical Profiles of Mean Vertical Velocity....................................................... 61

Figure 40: Transverse Profiles of Mean Streamwise Velocity ............................................. 63

Figure 41: Transverse Profiles of Mean Transverse Velocity ............................................... 64

Figure 42: Transverse Profiles of Mean Vertical Velocity .................................................. 65

Figure 43: Streamwise Profiles of Mean Streamwise Velocity ............................................... 66

Figure 44: Streamwise Profiles of Mean Transverse Velocity ............................................... 67

Figure 45: Streamwise Profiles of Mean Vertical Velocity .................................................... 68

Figure 46: Root Mean Square Streamwise Velocity Profiles ............................................... 69

Figure 47: Root Mean Square Streamwise Velocity Profiles (Nezu and Rodi, 1986) ............... 70

Figure 48: Root Mean Square Transverse Velocity Profiles ................................................... 71

Figure 49: Root Mean Square Vertical Velocity Profiles .................................................. 72

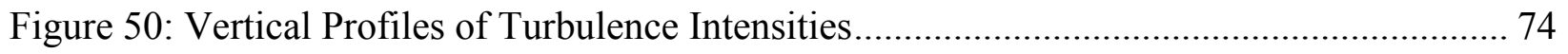

Figure 51: Transverse Profile of Turbulence Intensities..................................................... 75

Figure 52: Streamwise Profiles of Turbulence Intensities..................................................... 76

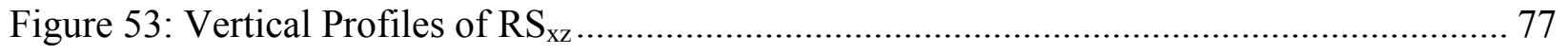

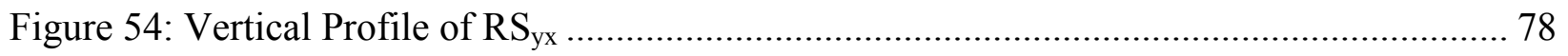

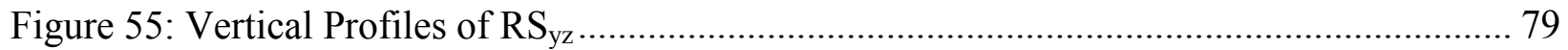

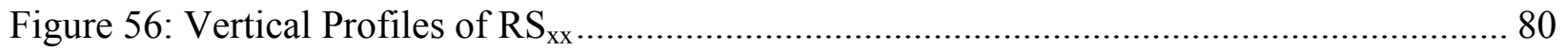




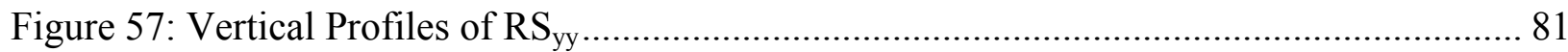

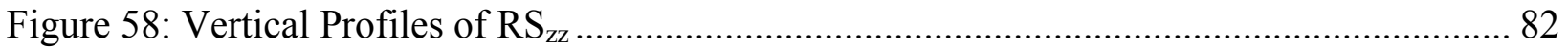

Figure 59: Energy Spectrum at $10 \mathrm{~cm}$ from Bottom of Quiescent Zone from Measurements on

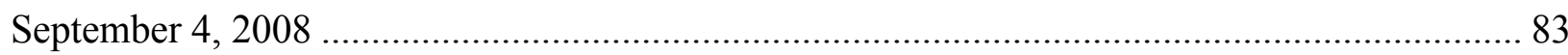

Figure 60: Energy Spectrum at $43 \mathrm{~cm}$ from Bottom of Quiescent Zone from Measurements on

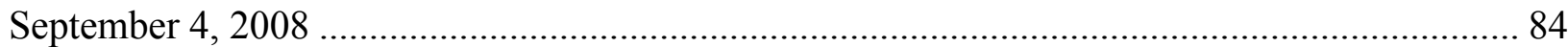

Figure 61: Energy Spectrum at $61 \mathrm{~cm}$ from Bottom of Quiescent Zone from Measurements on

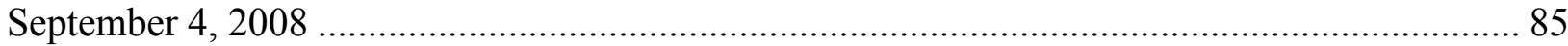

Figure 62: Energy Spectrum at $79 \mathrm{~cm}$ from Bottom of Quiescent Zone from Measurements on

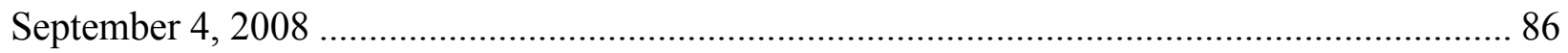

Figure 63: Energy Spectrum Measured in a Flume (Throwbidge et al., 1989) ............................ 87

Figure 64: Standpipe in Dry Quiescent Zone at Dogwood Lake................................................. 93

Figure 65: Piping from the Current Standpipe System at Dogwood Lake ................................... 93

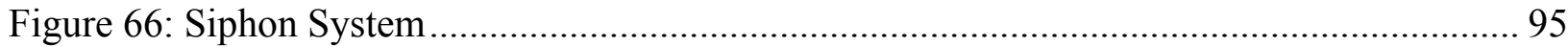

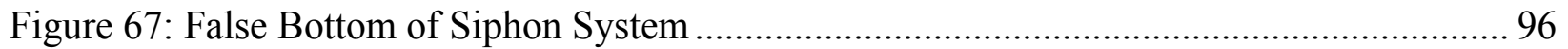

Figure 68: Lil Shark Automatic Pool Cleaner (1800Pools.com) …………………….................. 96

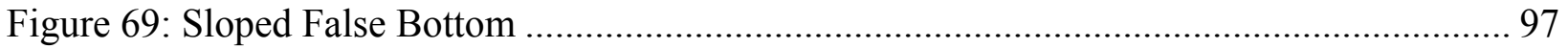

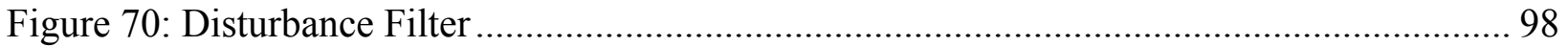

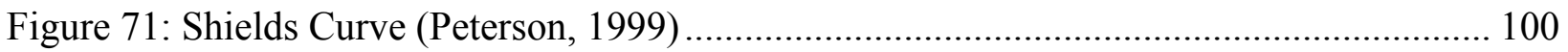

Figure 72: Free Body Diagram of a Particle on a Sloped Surface without Resistance of Water 102

Figure 73: Free Body Diagram of Particle on a Sloped Surface with Resistance of Water ....... 103

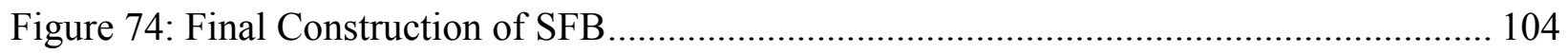

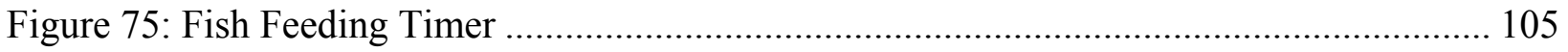

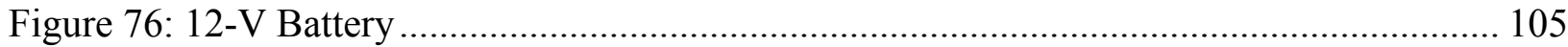

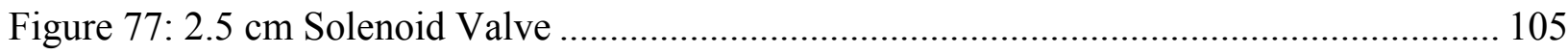

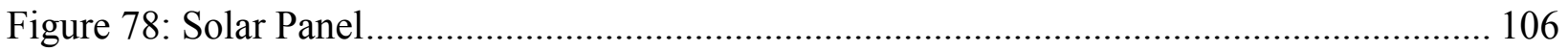

Figure 79: Waterproof Cases for Timing System and Solar Panel .............................................. 106

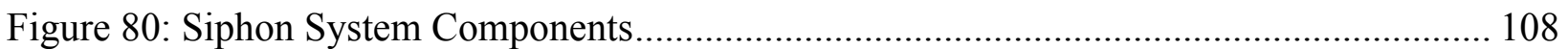

Figure 81: Piping from Siphon Tub to False Bottom ........................................................... 108

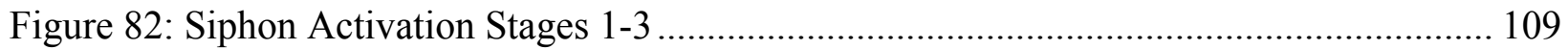

Figure 83: Side View of Siphon Activation (Stage 3) .......................................................... 109 
Figure 84: Side View of Siphon Deactivation (Stage 4)........................................................ 110

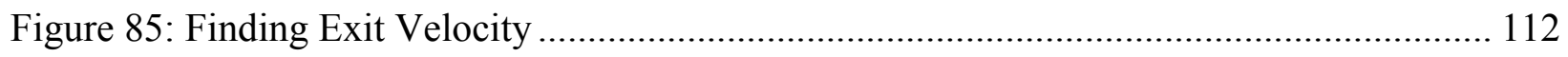

Figure 86: Determining Coverage Radius and Number of Holes Required for False Bottom ... 115

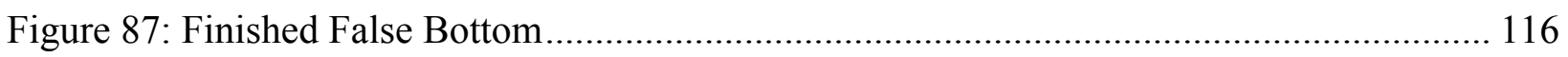

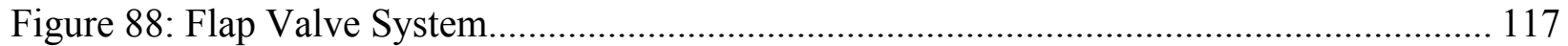

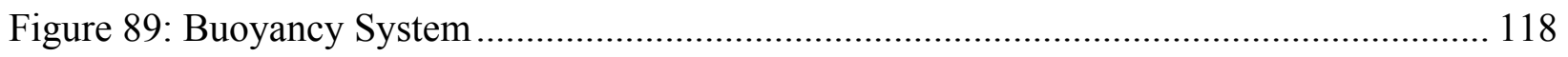

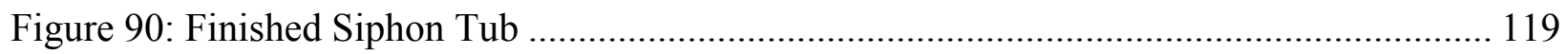

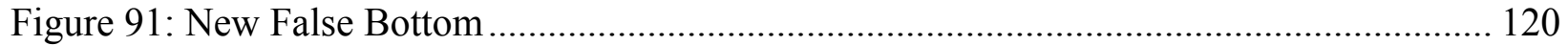

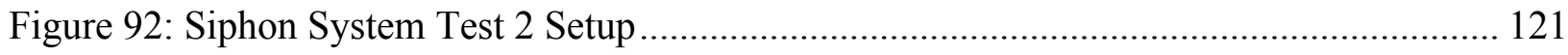

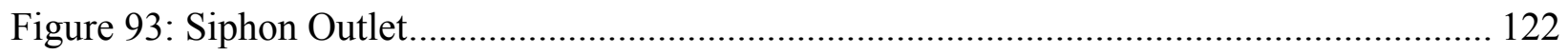

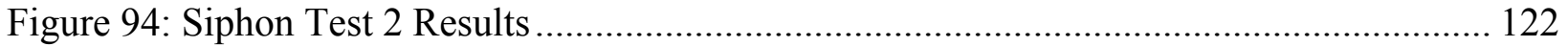

Figure 95: August 30 Vertical Profile of Mean Streamwise Velocity +/- One Standard Deviation

Figure 96: August 30 Vertical Profile of Mean Transverse Velocity +/- One Standard Deviation

Figure 97: August 30 Vertical Profile of Mean Vertical Velocity +/- One Standard Deviation 156 Figure 98: September 4 Vertical Profile of Mean Streamwise Velocity +/- One Standard Deviation.

Figure 99: September 4 Vertical Profile of Mean Transverse Velocity +/- One Standard

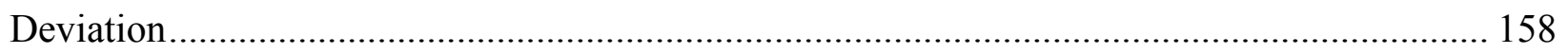

Figure 100: September 4 Vertical Profile of Mean Vertical Velocity +/- One Standard Deviation

Figure 101: September 30 Vertical Profile of Mean Streamwise Velocity +/- One Standard

Deviation 160

Figure 102: September 30 Vertical Profile of Mean Transverse Velocity +/- One Standard

Deviation... 161

Figure 103: September 30 Vertical Profile of Mean Vertical Velocity +/- One Standard Deviation 162

Figure 104: October 9 Vertical Profile of Mean Streamwise Velocity +/- One Standard Deviation 163 
Figure 105: October 9 Vertical Profile of Mean Transverse Velocity +/- One Standard Deviation 164

Figure 106: October 9 Vertical Profile of Mean Vertical Velocity +/- One Standard Deviation 165 Figure 107: November 4 Transverse Profile of Mean Streamwise Velocity +/- One Standard

Deviation 166

Figure 108: November 4 Transverse Profile of Mean Transverse Velocity +/- One Standard

Deviation. 167

Figure 109: November 4 Transverse Profile of Mean Vertical Velocity +/- One Standard Deviation 168

Figure 110: November 6 Transverse Profile of Mean Streamwise Velocity +/- One Standard Deviation 169

Figure 111: November 6 Transverse Profile of Mean Transverse Velocity +/- One Standard Deviation

Figure 112: November 6 Transverse Profile of Mean Vertical Velocity +/- One Standard Deviation

Figure 113: November 26 Streamwise Profile of Mean Streamwise Velocity +/- One Standard

Deviation... 172

Figure 114: November 26 Streamwise Profile of Mean Transverse Velocity +/- One Standard Deviation.

Figure 115: November 26 Streamwise Profile of Mean Vertical Velocity +/- One Standard

Deviation

Figure 116: November 29 Streamwise Profile of Mean Streamwise Velocity +/- One Standard

Deviation. 175

Figure 117: November 29 Streamwise Profile of Mean Transverse Velocity +/- One Standard Deviation 176

Figure 118: November 29 Streamwise Profile of Mean Vertical Velocity +/- One Standard Deviation. 177

Figure 119: Transverse Profiles of Root Mean Square Streamwise Velocity ......................... 178

Figure 120: Transverse Profiles of Root Mean Square Transverse Velocity .......................... 179

Figure 121: Transverse Profiles of Root Mean Square Vertical Velocity ............................... 180

Figure 122: Streamwise Profiles of Root Mean Square Streamwise Velocity 181 
Figure 123: Streamwise Profiles of Root Mean Square Transverse Velocity .......................... 182

Figure 124: Streamwise Profiles of Root Mean Square Vertical Velocity .............................. 183

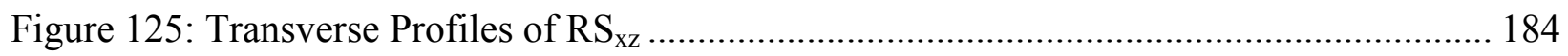

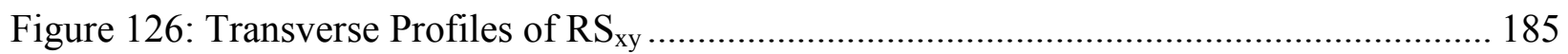

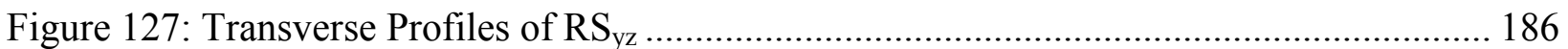

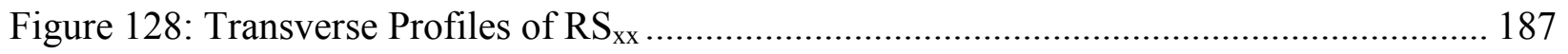

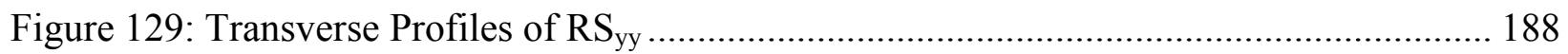

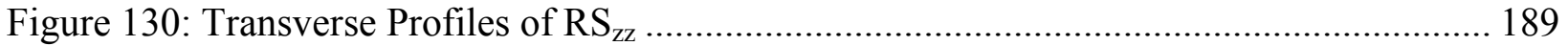

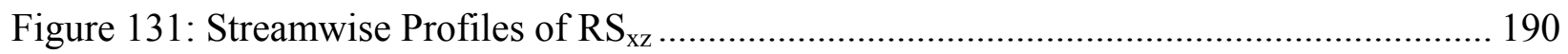

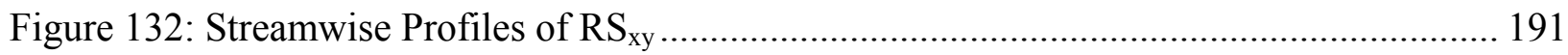

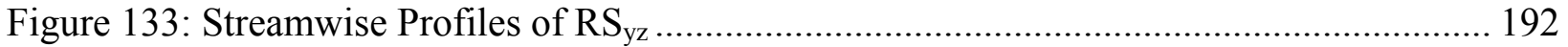

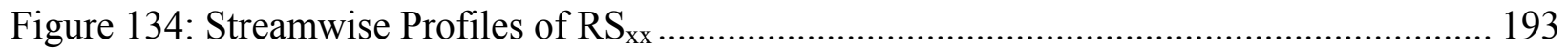

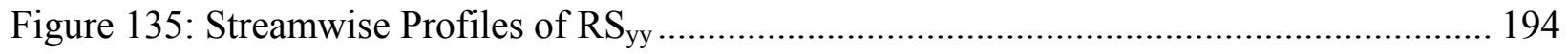

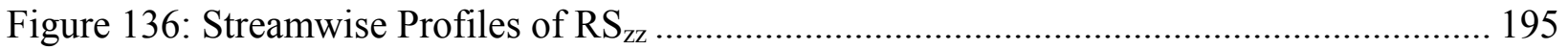




\section{List of Tables}

Table 1: Dogwood Lake Raceway Dimensions ................................................................. 2

Table 2: Micro ADV Velocity Ranges ................................................................................. 31

Table 3: Error in Measured Characteristics Due to ADV Uncertainty ..................................... 34

Table 4: Streamwise Traversing Mechanism Uncertainty ....................................................... 38

Table 5: Transverse Traversing Mechanism Uncertainty ........................................................ 38

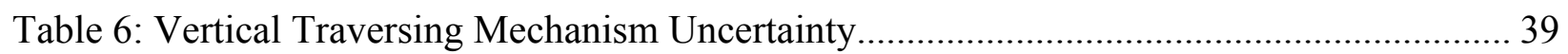

Table 7: Angular Sensitivity with Respect to Small Rotations around the Z-Axis ................... 43

Table 8: Description of Collected Measurements................................................................. 46

Table 9: Rating of Basic Requirements for Design Ideas...................................................... 98

Table 10: Velocity Measurements Taken on August 30, 2008........................................... 127

Table 11: Mean Velocity Values Measured on August 30, 2008 ......................................... 128

Table 12: Standard Deviation and SNR Measured on August 30, 2008 ................................. 129

Table 13: Reynolds Stress, TI, and RMS Measured on August 30, 3009 ............................. 130

Table 14: Velocity Measurements Taken on September 4, 2008 ........................................... 131

Table 15: Mean Velocity Values Measured on September 4, 2008 ...................................... 132

Table 16: Standard Deviation and SNR Measured on September 4, 2008 ............................. 133

Table 17: Reynolds Stress, TI, and RMS Measured on September 4, 2008 ........................... 134

Table 18: Velocity Measurements Taken on September 30, 2008 ...................................... 135

Table 19: Mean Velocity Values Measured on September 30, 2008 .................................... 135

Table 20: Standard Deviation and SNR Measured on September 30, 2008 .......................... 136

Table 21: Reynolds Stress, TI, and RMS Measured on September 30, 2008 ........................ 136

Table 22: Velocity Measurements Take on October 9, 2008 ............................................. 137

Table 23: Mean Velocity Values Measured on October 9, 2008 .......................................... 137

Table 24: Standard Deviation and SNR Measured on October 9, 2008 ................................ 138

Table 25: Reynolds Stress, TI, and RMS Measured on October 9, 2008 .............................. 139

Table 26: Velocity Measurements Taken on November 4, 2008 ......................................... 140

Table 27: Mean Velocity Values Measured on November 4, 2008...................................... 141

Table 28: Standard Deviation and SNR Measured on November 4, 2008 ............................. 142

Table 29: Reynolds Stress, TI, and RMS Measured on November 4, 2008 ........................... 143

Table 30: Velocity Measurements Taken on November 6, 2008 ......................................... 144 
Table 31: Mean Velocity Values Measured on November 6, 2008..................................... 145

Table 32: Standard Deviation and SNR Measured on November 6, 2008 ............................ 146

Table 33: Reynolds Stress, TI, and RMS Measured on November 6, 2008 .......................... 147

Table 34: Velocity Measurements Taken on November 26, 2008 ........................................ 148

Table 35: Mean Velocity Values Measured on November 26, 2008..................................... 148

Table 36: Standard Deviation and SNR Measured on November 26, 2008 .......................... 149

Table 37: Reynolds Stress, TI, and RMS Measured on November 26, 2008 ........................ 150

Table 38: Velocity Measurements Taken on November 29, 2008 ...................................... 151

Table 39: Mean Velocity Values Measured on November 29, 2008 .................................... 151

Table 40: Standard Deviation and SNR Measured on November 29, 2008 .......................... 152

Table 41: Reynolds Stress, TI, and RMS Measured on November 29, 2008 ......................... 153 


\section{List of Symbols}

Symbol

$\alpha$

$\beta$

$\Delta$

$\delta$

$\varepsilon$

$\theta$

$\Theta_{\text {critical }}$

$\theta$

$\theta_{\text {Critical }}$

$\lambda_{u}$

$\lambda_{w}$

$\mu_{w}$

$v_{w}$

$\rho_{w}$

$\bar{\tau}$

$\overline{\tau_{w}}$

$\Phi_{S_{11}}$

$\Phi S_{22}$

$\Phi S_{33}$

$\Phi t_{11}$

\section{Description}

Empirical Constant in Equation 47

Empirical Constant in Equation 33

Change in

Relating to Partial Differential

Turbulent Kinetic Energy Dissipation

Mobility Number

Critical Mobility Number

Angle of Slopes

Critical Angle of Slopes

Empirical Constant in Equation 16

Empirical Constant in Equation 17

Dynamic Viscosity of Water

Kinematic Viscosity of Water

Density of Water

Total Mean Shear Stress

Mean Wall Shear Stress

Wave-number Spectrum Relating to Streamwise Fluctuations

Wave-number Spectrum Relating to Transverse Fluctuations

Wave-number Spectrum Relating to Vertical Fluctuations

Frequency Spectrum Relating to Streamwise Fluctuations 
Symbol

\begin{tabular}{|c|c|}
\hline$\Phi t_{22}$ & Frequency Spectrum Relating to Transverse Fluctuations \\
\hline$\Phi t_{33}$ & Frequency Spectrum Relating to Vertical Fluctuations \\
\hline$A$ & Cross Sectional Area of Open Channel \\
\hline$A_{p}$ & Cross Sectional Area of Spherical Particle \\
\hline$a$ & Acceleration \\
\hline$C$ & Speed of Sound Wave \\
\hline$D_{u}$ & Empirical Constant in Equation 16 \\
\hline$D_{w}$ & Empirical Constant in Equation 17 \\
\hline$d$ & Diameter of Spherical Particle \\
\hline$d_{R}$ & Diameter of Sampling Volume \\
\hline$F$ & ADV Performance Factor \\
\hline$F 1$ & Hydrostatic Force \\
\hline$F 2$ & Hydrostatic Force \\
\hline$F_{g x}$ & Force of Gravity Acting in $x$-direction \\
\hline$F_{v x}$ & Total Shear Force Acting in $x$-direction \\
\hline$F_{w}$ & Force of Moving Water on Spherical Particle \\
\hline$F_{x}$ & Force Acting in $x$-direction \\
\hline$f$ & Frequency \\
\hline$g$ & Acceleration due to Gravity \\
\hline$H_{s p}$ & Vertical Length of Siphon Pipe \\
\hline
\end{tabular}

\section{Description}




\section{Description}

\begin{tabular}{|c|c|}
\hline$h$ & Depth of Water in Quiescent Zone \\
\hline$k$ & Wave-number \\
\hline$k_{1}$ & Wave-number Relating to Streamwise Fluctuations \\
\hline$k_{2}$ & Wave-number Relating to Transverse Fluctuations \\
\hline$k_{3}$ & Wave-number Relating to Vertical Fluctuations \\
\hline$m$ & Mass \\
\hline$n$ & Number of Samples Measured by ADV \\
\hline $\bar{P}$ & Mean Pressure \\
\hline$P_{t}$ & Time Between Fluctuations \\
\hline$P_{w}$ & Wetted Perimeter of Channel \\
\hline$Q$ & Volumetric Flow Rate \\
\hline$Q_{\text {critical }}$ & Critical Flow Rate \\
\hline$R D_{11}$ & Spatial Correlation Function Relating to Streamwise Fluctuations \\
\hline$R D_{22}$ & Spatial Correlation Function Relating to Transverse Fluctuations \\
\hline$R D_{33}$ & Spatial Correlation Function Relating to Vertical Fluctuations \\
\hline$R T_{11}$ & Temporal Correlation Function Relating to Streamwise Fluctuations \\
\hline$R T_{22}$ & Temporal Correlation Function Relating to Transverse Fluctuations \\
\hline$R T_{33}$ & Temporal Correlation Function Relating to Vertical Fluctuations \\
\hline$R S_{x x}$ & Reynolds Stress \\
\hline$R S_{x y}$ & Reynolds Stress \\
\hline$R S_{x z}$ & Reynolds Stress \\
\hline
\end{tabular}




\begin{tabular}{|c|c|}
\hline$R S_{y y}$ & Reynolds Stress \\
\hline$R S_{y z}$ & Reynolds Stress \\
\hline$R S_{z z}$ & Reynolds Stress \\
\hline $\boldsymbol{r}$ & Spatial Vector \\
\hline$r_{1}$ & Distance in $x$-direction Relating to Spatial Correlation Function \\
\hline$r_{2}$ & Distance in $y$-direction Relating to Spatial Correlation Function \\
\hline$r_{3}$ & Distance in $z$-direction Relating to Spatial Correlation Function \\
\hline$r$ & Radial Distance \\
\hline$t$ & Time \\
\hline $\mathrm{U}$ & Magnitude of Velocity Vector Resultant \\
\hline$u$ & Streamwise Velocity \\
\hline $\bar{u}$ & Mean Streamwise Velocity \\
\hline$u^{\prime}$ & Fluctuations in Streamwise Velocities \\
\hline$u_{R M S}$ & Root Mean Square Streamwise Velocity \\
\hline$u_{*}$ & Friction Velocity \\
\hline$v$ & Transverse Velocity \\
\hline $\bar{v}$ & Mean Transverse Velocity \\
\hline$v^{\prime}$ & Fluctuations in Transverse Velocities \\
\hline$v_{R M S}$ & Root Mean Square Streamwise Velocity \\
\hline$w$ & Vertical Velocity \\
\hline
\end{tabular}


Symbol

$\bar{W}$

$w^{\prime}$

$w_{R M S}$

\section{Description}

Mean Vertical Velocity

Fluctuations in Vertical Velocities

Root Mean Square Vertical Velocity 


\section{Part 1: Characteristics of Turbulence in the Quiescent Zone}

\subsection{Introduction}

An aquaculture raceway is a water carrying open channel with a rectangular cross-section used to raise fish. The quiescent zone of an aquaculture raceway is the most downstream section of the raceway that allows solids such as fish waste and organic matter to settle. The quiescent zone is separated from the fish to reduce the level of turbulence which in turn provides adequate flow characteristics for solid settling to occur.

\subsubsection{Dogwood Lake’s Raceway System}

West Virginia is known for many things, but most famous are its coal mines. Over time, these underground mines collect gravity flow sources of water, often with ideal conditions for raising fish (Miller, 2008). Dogwood Lake located in Monongahela County West Virginia was formed as a polishing pond for the mine water from Consol Energy to settle. This water contains high levels of contaminants such as iron, aluminum, and sulfate which must first be treated to meet U.S. pollution standards before entering the lake (Slagle, 2006). A subsequent raceway system was built in 2002 by West Virginia University utilizing the effluence from the lake. Due to the $\mathrm{pH}$, temperature, and absence of harmful bacteria in the water, it was deemed ideal for raising certain types of fish such as trout, catfish, and bass (Miller, 2008). This multi-purpose aquaculture raceway not only serves as an ideal environment for raising fish, but also as a source of reclamation for mine water that would otherwise be discharged into the natural environment.

The rectangular aquaculture raceways studied in this research are located at Dogwood Lake. The flow through Dogwood Lake's raceway system has a Reynolds number of approximately $2 \times 10^{4}$ and contains subcritical flow. The system is comprised of four levels each consisting of two raceways in parallel. Each raceway has an inlet, fish, and quiescent zone as shown in Figure 1 and each section is separated by a screen. Starting at the beginning of the system, water from an upstream reservoir (Dogwood Lake) enters a head box where it is distributed to two parallel raceways. The water travels through an inlet zone and a screen. This screen prevents fish from entering the inlet zone. Next, the water enters the fish zone (where the 
fish are located). A screen is located at the end of this zone to prevent fish from entering the quiescent zone. Finally, the water enters the quiescent zone and then flows over a weir and drops approximately $119 \mathrm{~cm}$ to the next two parallel raceways. Figure 2 shows a top and side view of the four levels at Dogwood Lake. Each raceway is labeled with a letter (which distinguishes each parallel raceway) followed by a number (representing the level).

Dimension ranges of the raceway system at Dogwood Lake can be seen in Figure 1 and Table 1. These ranges are due to slight variations from one raceway to the next. The coordinate system in Figure 1 illustrates the orientation of the system where the positive streamwise direction corresponds to $+\mathrm{X}$, the positive transverse direction corresponds to $+\mathrm{Y}$, and the positive vertical direction corresponds to $+Z$. The mean velocities in each direction (streamwise, transverse, and vertical) are denoted using $\bar{u}, \bar{v}$, and $\bar{w}$ respectively. It should be noted that the bottom slope (Theta) at Dogwood Lake is very small.

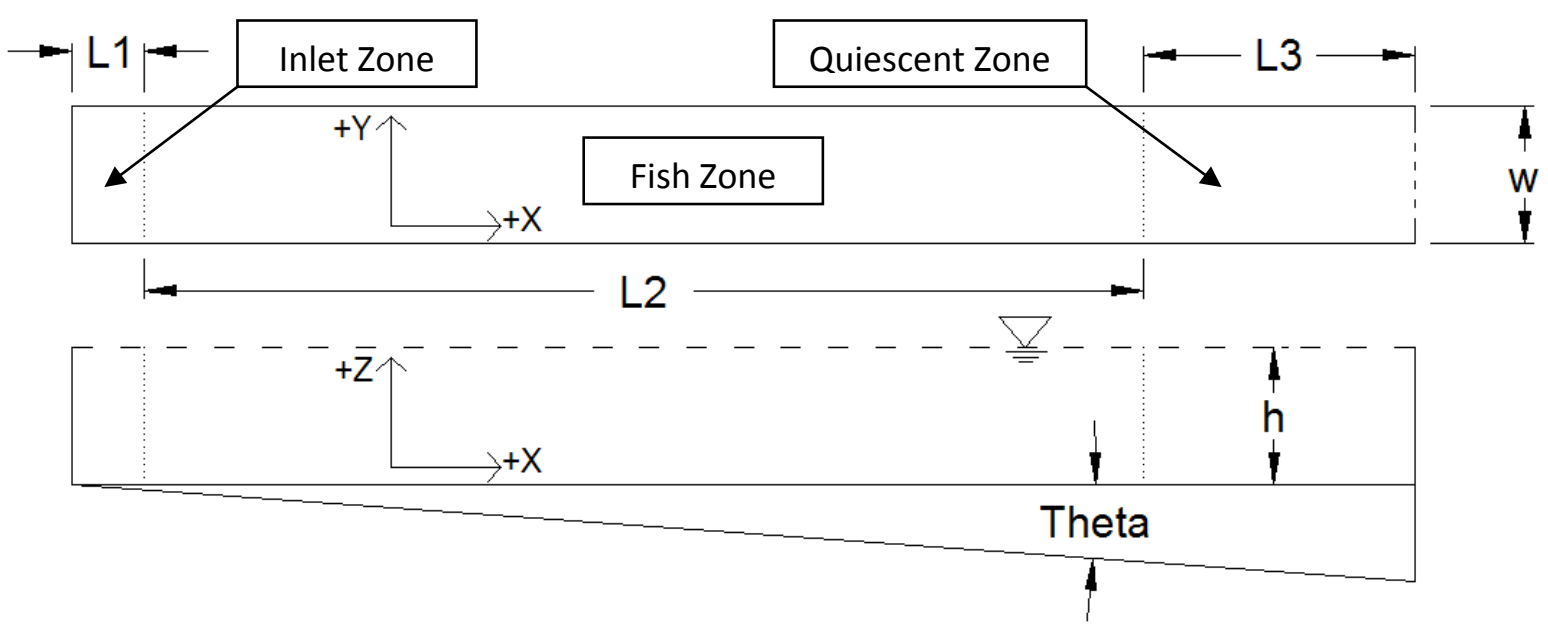

Figure 1: Dimensions of Raceway at Dogwood Lake

Table 1: Dogwood Lake Raceway Dimensions

\begin{tabular}{|c|c|c|c|c|c|}
\hline L1(cm) & L2(cm) & L3(cm) & W(cm) & h(cm) & Theta(deg) \\
\hline $46-61$ & $630-670$ & $170-180$ & $86-96$ & $86-91$ & $0-1.8 \times 10^{-4}$ \\
\hline
\end{tabular}




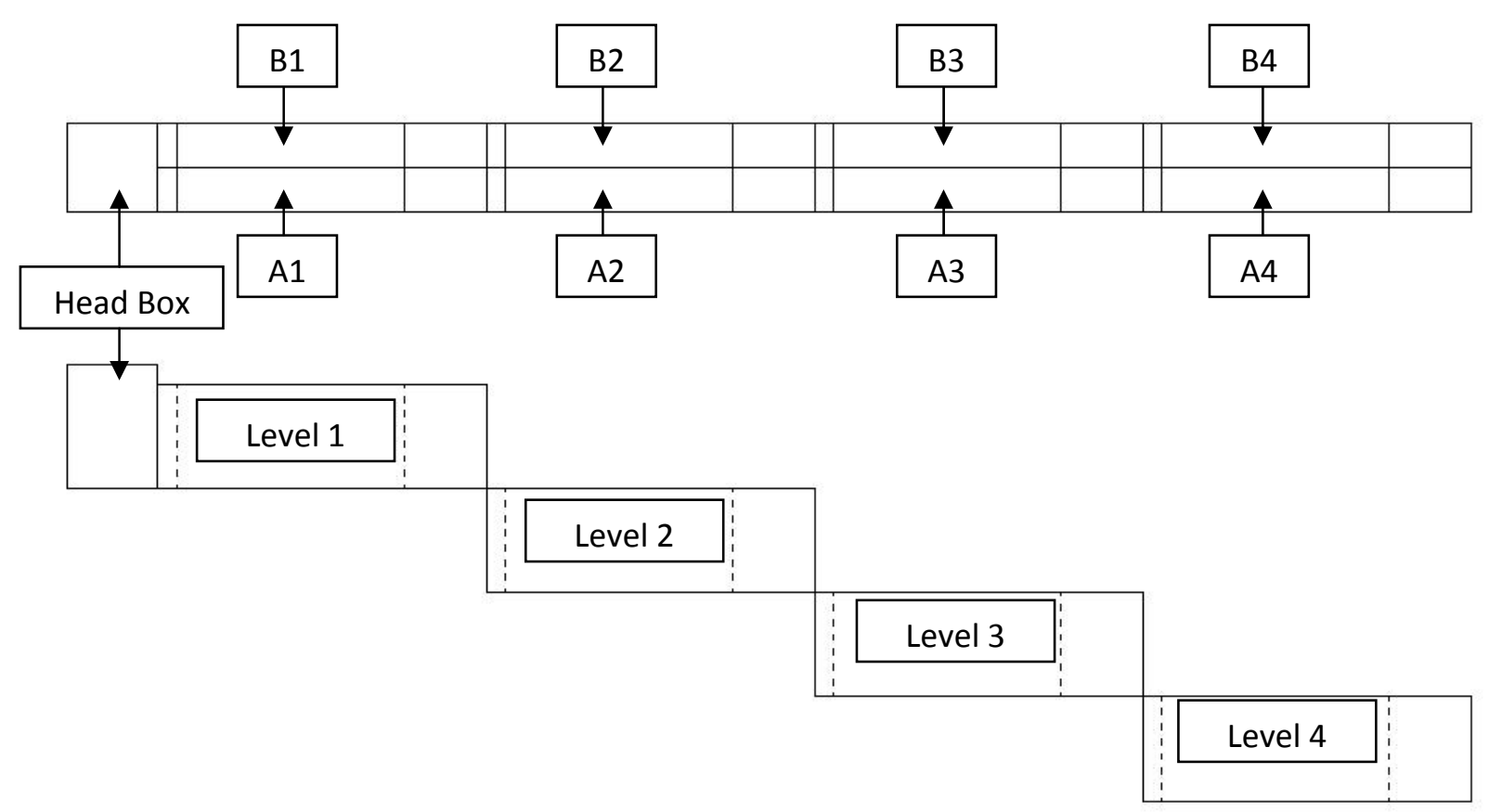

Figure 2: Dogwood Lake’s Raceway Setup

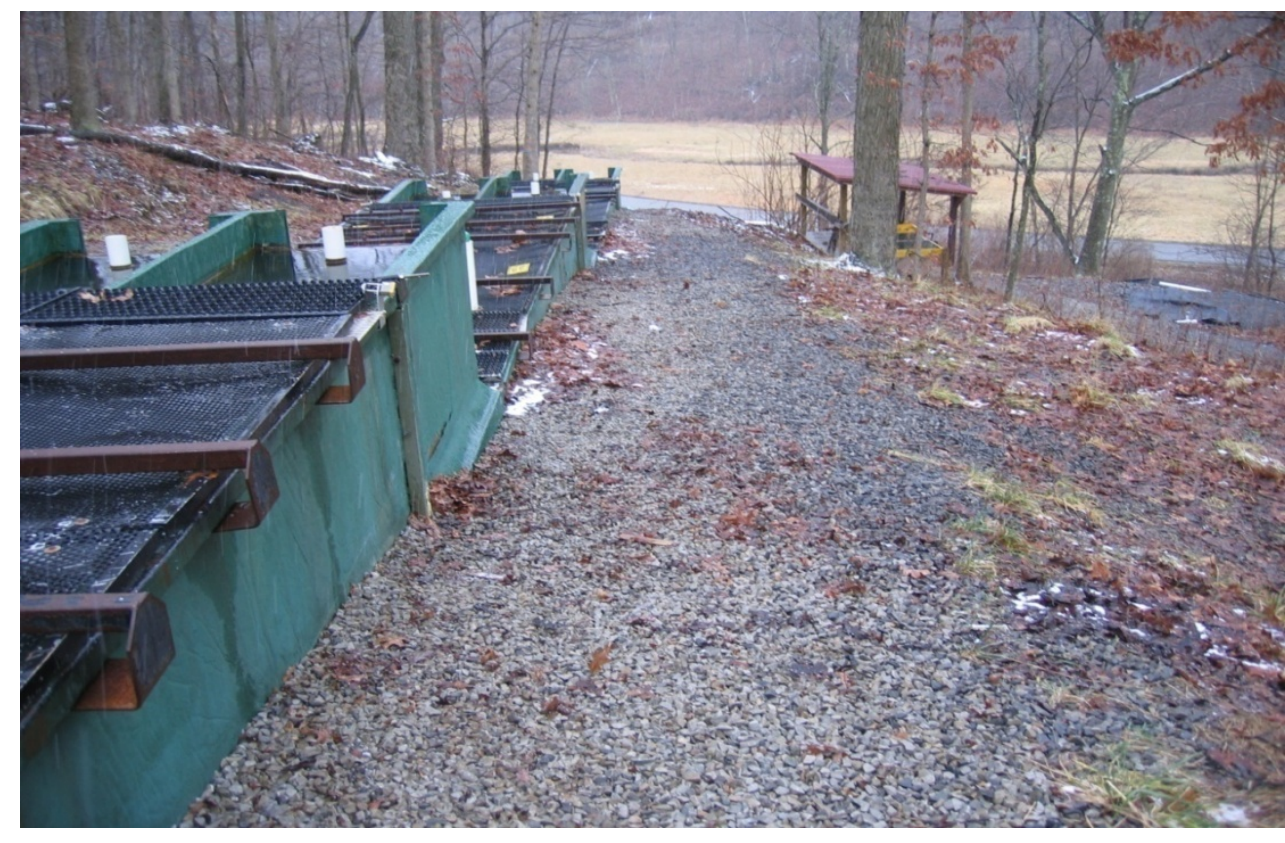

Figure 3: Downstream View of the Raceway System at Dogwood Lake 


\subsection{Research Objectives}

The first main objective of this research is to provide accurate velocity measurements that will describe the flow characteristics in different quiescent zones at Dogwood Lake, and determine if the flow through the quiescent zones contains two-dimensional, fully developed, turbulent open channel flow characteristics. Any discrepancies between the measured characteristics and two-dimensional, full developed, turbulent open channel characteristics will be analyzed.

Few velocity measurements have been taken in rectangular aquaculture raceway systems. Huggins (2003) and Rumberg (2004) have previously taken velocity measurements in a rectangular aquaculture raceway system; however, measurements were limited due to factors such as equipment used, averaging time, and large distances between measurements. Additionally, neither Huggins nor Rumberg described turbulence characteristics within the quiescent zone. This research intends to improve upon the velocity measurements taken by Huggins and Rumberg, and quantify different turbulence characteristics within the quiescent zone. This is accomplished by using a more sophisticated device for measuring velocities, averaging mean velocities over a longer sample period, collecting more measurements within a given distance, and deriving turbulence characteristics from the measured velocities.

To achieve this, velocity measurements are taken in different quiescent zones with slightly different parameters. The velocity measurements are collected using SonTek's Micro Acoustic Doppler Velocimeter (ADV). Using these measurements, the following profiles are created: mean velocity profiles, turbulence intensity profiles, Reynolds stress profiles, and root mean square (RMS) profiles. These profiles are taken in the vertical, transverse, and streamwise directions. They are compared to theoretical/empirical calculations for two-dimensional, fully developed, open channel, turbulent flow over smooth surfaces. Comparison of the theoretical/empirical profiles to the measured profiles will reveal the effect of disturbances such as the presence of fish and screens that are located upstream of the quiescent zone. When comparing measurements in different quiescent zones, the effect of the different parameters on the flow characteristics may become visible. 


\subsection{Literature Review}

\subsubsection{Introduction to Open Channel Flow}

Open channel flow has been studied in detail for many years. From these studies several theoretical/empirical equations have been developed to characterize the flow through open channels. Most of this research has been done using laboratory flumes where the flow properties are controllable. In addition, disturbances such as wind shear and obstacles in the flow are limited in laboratory environments to provide results that are dependent on the least amount of variables. Studies by Trowbridge et al. (1989) show that theoretical/empirical equations can be used to closely match the results of measurements taken in a laboratory flume. These results are important when determining the specific requirements of the open channel (i.e. length, width) that create good agreement with the theoretical/empirical equations.

Measurements have also been taken in rectangular aquaculture raceway systems. These measurements are limited and will be used for this study to show improvements that should be made to obtain a better description of the flow characteristics within the quiescent zone of an aquaculture raceway.

\subsubsection{Introduction to Measuring Open Channel Flow}

Characteristics of open channel flow are determined by measuring the velocities at different points in the channel. Measuring open channel flow is a difficult task considering that most flows through open channels are turbulent (containing random fluctuations in all three components of velocity). Turbulent flows require instruments that can measure velocities at a fast rate in order to capture the fluctuations in the velocities. Laser Doppler Velocimeters (LDV), Electromagnetic Current Meters (ECM), Hot-wire Anemometers (HWA), and Particle Tracking Velocimeters (PTV) are commonly used to measure flow velocities in open channels. However, these instruments are not practical for measuring velocities in field environments. Limitations such as ruggedness, required calibration, range limitation due to turbid waters (LDV), and complex setup create problems when using these instruments in the field. In addition, the cost of these instruments is relatively high. Considering the previous complications, the Acoustic Doppler Velocimeter (ADV) was chosen as the most suitable instrument to measure velocities in the quiescent zones at Dogwood Lake. The ADV is rugged 
enough to handle field environments, samples at relatively high frequencies, requires a simple setup to measure velocities, requires no calibration, and is cost effective. Research has been conducted to determine the ability of the ADV to measure turbulence. This research is vital to validate the accuracy of the ADV for measuring open channel flow.

\subsubsection{Development of the ADV}

The ADV was developed by the U.S. Army at the U.S. Army Engineer Waterways Experiment Station (WES) in Vicksburg, Mississippi (Kraus et al, 1994). WES is comprised of several facilities used for basic hydraulic and wave research studies. The motivation for the development of the ADV was to create an accurate velocity measuring system that is rugged enough to withstand atmospheric conditions. The U.S. Army required the ADV to meet the following basic requirements: relatively inexpensive (less than $\$ 10,000$ ), easily transportable, have mechanical ruggedness, have environmental ruggedness (in order to endure changes in temperature and humidity), relatively small size so the flow would not be disturbed, eliminate frequent calibration, use normal power while limiting safety hazards, and be capable of interfacing with a wide-range of personal computers (Kraus et al, 1994). The final result was an ADV probe with a $7 \mathrm{~mm}$ diameter and $40 \mathrm{~cm}$ long stem. At the end of the stem are three receive transducers placed at $120^{\circ}$ azimuth angles and angled $30^{\circ}$ from the transmitter (Figure 4). 


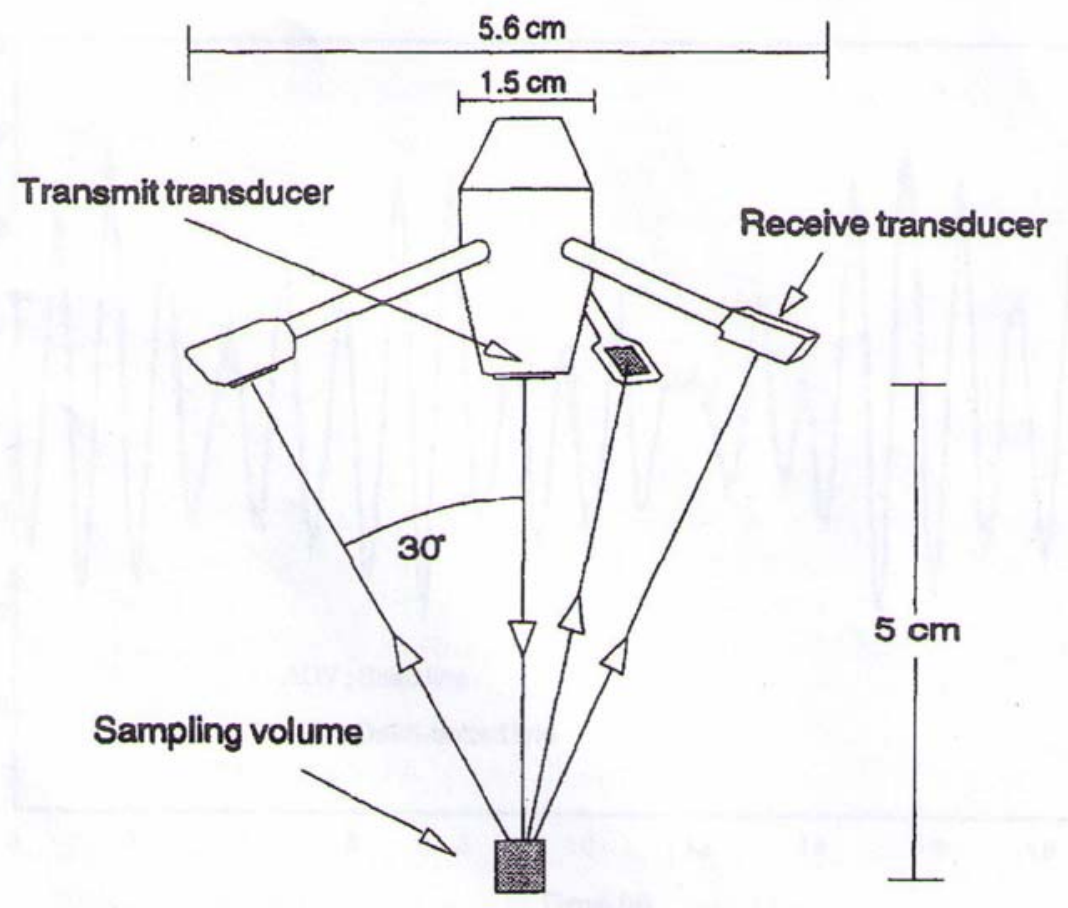

Figure 4: ADV Probe Tip Developed by Kraus et al. (1994)

This ADV has a controllable sampling rate $(0-25 \mathrm{~Hz}), 0-40^{\circ} \mathrm{C}$ operating temperature range, an accuracy of $\pm 0.25 \mathrm{~cm} / \mathrm{s}$, a resolution of $0.01 \mathrm{~cm} / \mathrm{s}$, a velocity range of $0-2.5 \mathrm{~m} / \mathrm{s}$, and transmits a narrow sound wave beam at $10 \mathrm{MHz}$ to a sampling volume located $5 \mathrm{~cm}$ from the transmitter (Kraus et al., 1994). Several tests were conducted to determine the ability of the ADV. One of the tests compared horizontal velocities measured by the three-dimensional ADV and a twodimensional LDV under generated surface waves with a period of 1.0 second. Figure 5 shows the results of this test. These results show good agreement between the two devices. Results from linear regression showed an offset of $0.11 \mathrm{~cm} / \mathrm{s}$. 


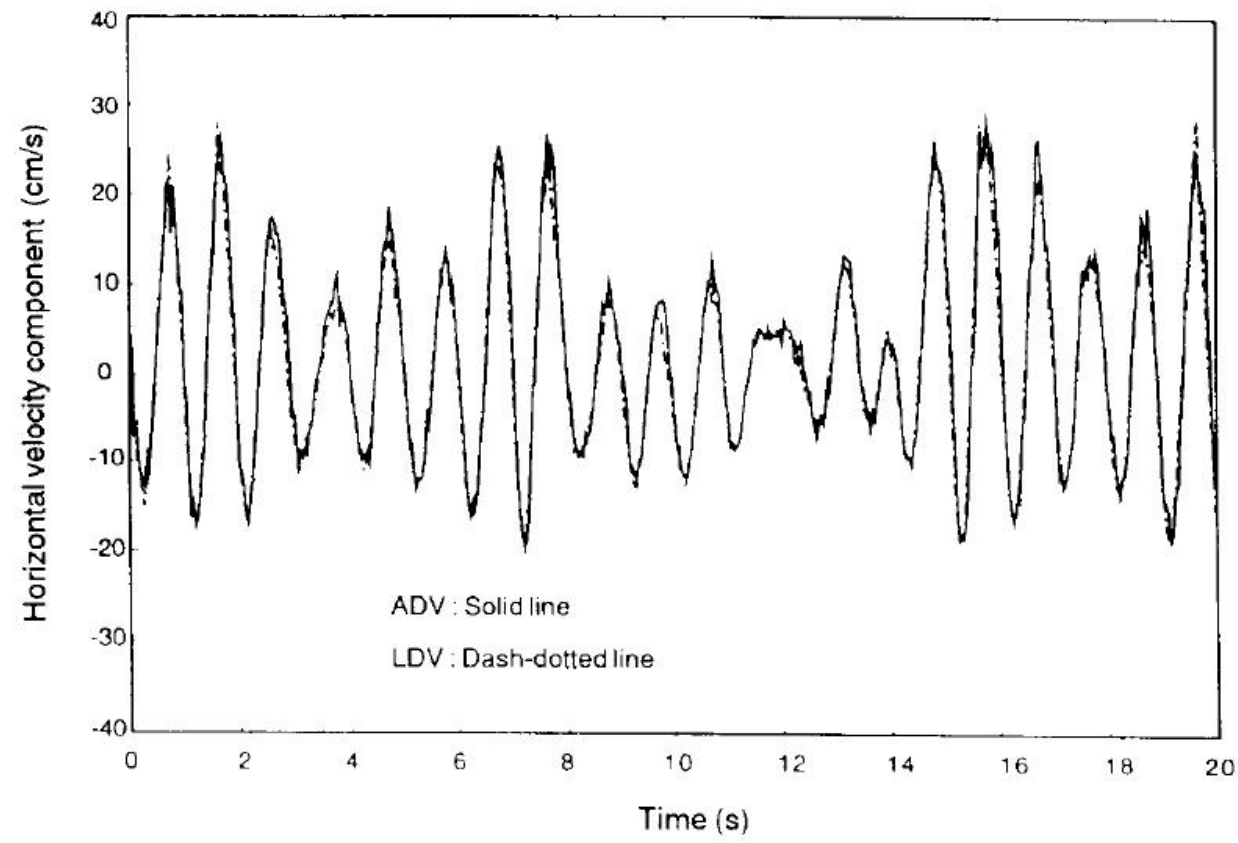

Figure 5: Horizontal Velocity Measurement Comparison between ADV and LDV (Kraus et al., 1993)

\subsubsection{Previous Research on Open Channel Flow}

\subsubsection{LDV Velocity Measurements in a 17 Meter Flume}

Trowbridge et al. (1989) used a Laser Doppler Velocimeter to measure flow characteristics in a $17 \mathrm{~m}$ long, $60 \mathrm{~cm}$ wide, and $30 \mathrm{~cm}$ deep flume. The objective of this research was to determine if the flume is capable of producing two-dimensional, fully developed, open channel, turbulent flow characteristics near the center of the flume. Two-dimensional, fully developed, open channel flow is considered to have mean properties that are independent of time, transverse position near the center line, and streamwise position. Using velocity measurements from the LDV, Trowbridge et al. calculated vertical profiles of transverse velocities, Reynolds stresses (discussed in Section 4.1.2), and Root Mean Square (RMS) velocities (discussed in Section 4.1.0). Figures 6-8 show measured vertical profiles of the previously mentioned flow parameters and their values calculated using theoretical/empirical equations describing two-dimensional, fully developed flow. In each of Figures 6-8 the crosses represent the measured values, and the smooth curve represents the theoretical/empirical values 
used to describe the corresponding flow parameter. Figures 6-8 show that the $17 \mathrm{~m}$ flume is close to producing two-dimensional, fully developed, open channel, turbulent flow characteristics. Discrepancies exist in Figure 6 as the free surface is approached, and in Figures 7-8 due to the scatter in measured values. This research shows that theoretical/empirical equations can be used to describe turbulent flow in open channels with relatively good agreement. This research also demonstrates that discrepancies between the theoretical/empirical values and measured values are inevitable, even in laboratory environments.

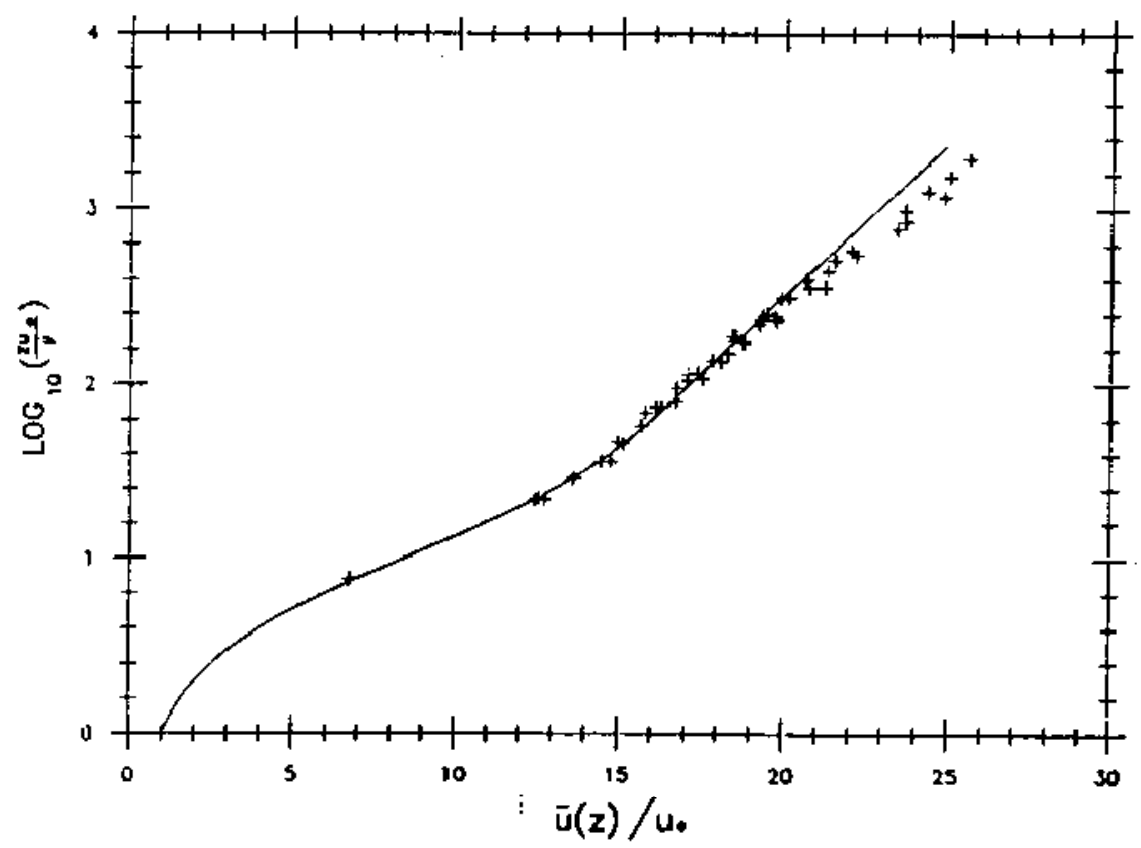

Figure 6: Dimensionless Semi-Log Vertical Profile of Mean Streamwise Velocity (Trowbridge et al., 1989) 


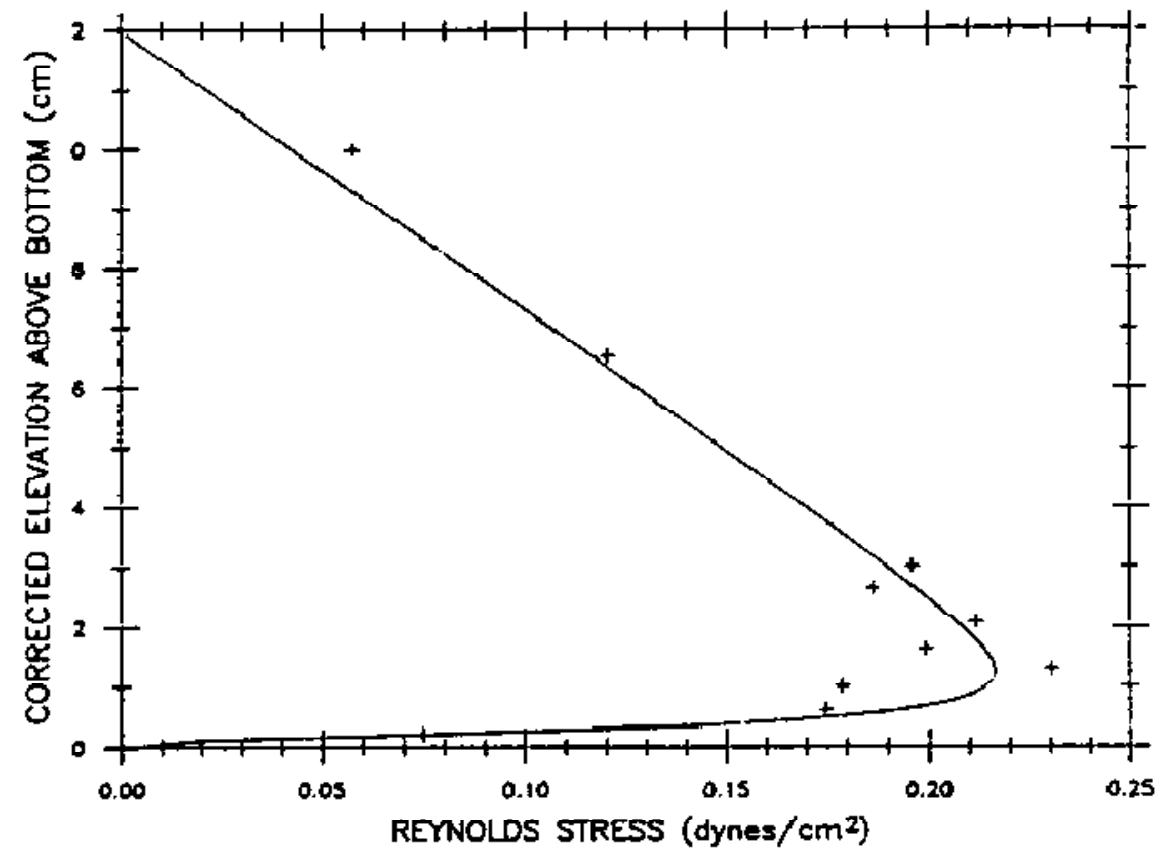

Figure 7: Vertical Profile of Reynolds Stresses (Trowbridge et al., 1989)

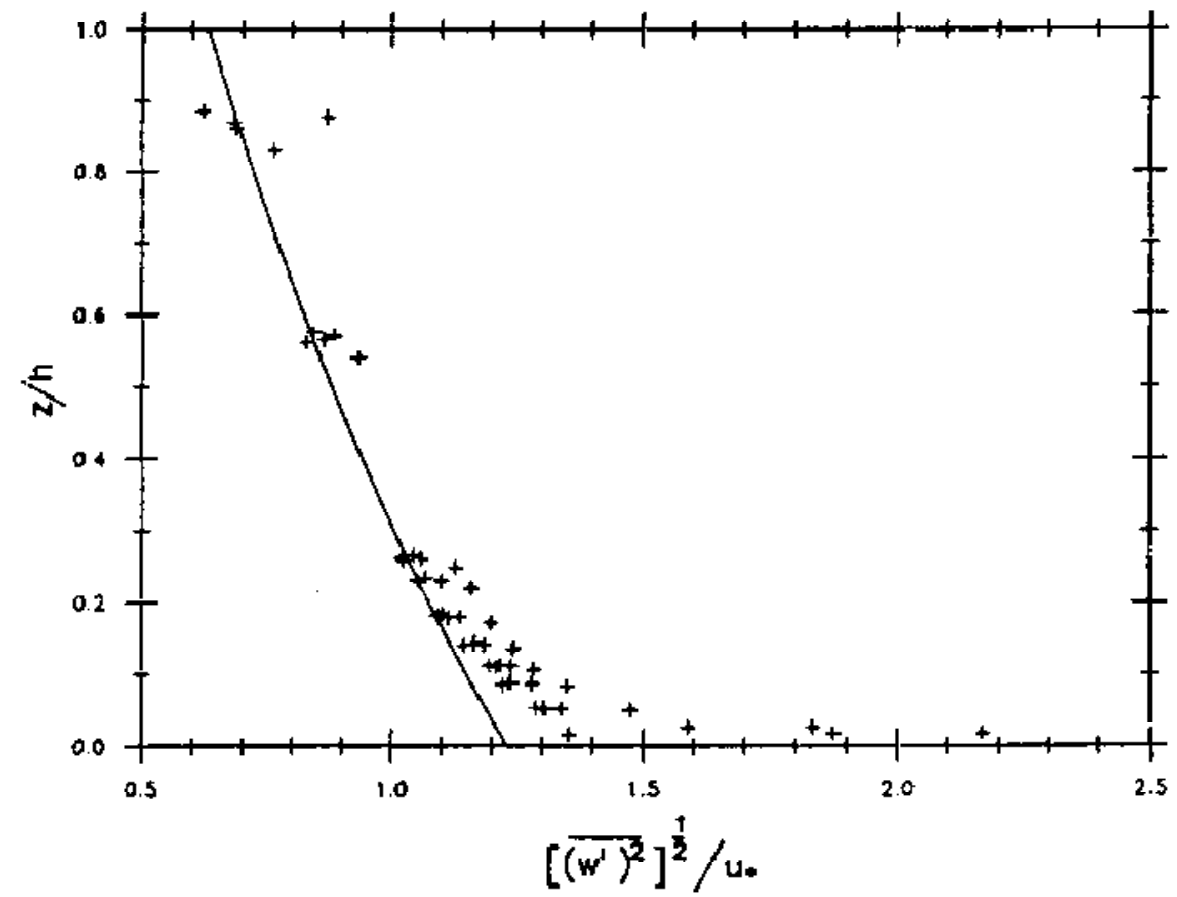

Figure 8: Dimensionless Vertical Profile of RMS Vertical Velocity (Trowbridge et al., 1989) 


\subsubsection{Open Channel Flow Measurements with a Laser Doppler Anemometer}

Nezu and Rodi (1986) used a laser Doppler anemometer to measure velocities in a $20 \mathrm{~m}$ long, $60 \mathrm{~cm}$ wide, and $65 \mathrm{~cm}$ deep flume. Their main objective was to re-examine the law of the wall and velocity defect log law. They arrived at several conclusions. First, when the log-law is restricted to a near-wall region, a von Karman constant of 0.412 and an integration constant of 5.29 can be used without considering the Froude or Reynolds number. Second, the friction velocity can be found accurately from velocity measurements using the previously stated values for the von Karman and integration constants. Third, the deviation from the log-law in the turbulent region should not be ignored. Coles' wake function can be used for deviations from the log-law. Finally, they found that deviations from the log-law in the turbulent region are vital for open channel flow at high Reynolds numbers and that turbulence intensities do not depend on the Reynolds number.

\subsubsection{Turbulent Open Channel Flow Over Smooth and Rough Boundaries}

Grass (1971) collected open channel flow characteristics within a $10 \mathrm{~m}$ long, $25 \mathrm{~cm}$ wide, and $5 \mathrm{~cm}$ deep glass channel. Grass used the hydrogen bubble technique along with a high speed camera in order to visualize and quantify turbulent characteristics in the flow. His objective was to determine the effects of surface roughness on the turbulence characteristics. Grass collected data in a channel with smooth, transitional, and rough bottom surfaces. He found that turbulence intensities resulting from fluctuations in the vertical direction are increased with surface roughness and turbulence intensities from fluctuations in the streamwise direction decrease with surface roughness. He also found that minimum streamwise velocities are related to regions of positive vertical velocities. Similarly, he found a relationship between local maximum streamwise velocities and regions of negative vertical velocities. He states that both of the

previous relationships contribute to positive Reynolds stresses within and near the boundary layer.

\subsubsection{ADV Measurements in Aquaculture Raceway Systems}

Rumberg (2004) took velocity measurements in rectangular aquaculture raceways at Dogwood Lake using an ADV. Rumberg's objectives were to describe the hydrodynamic 
properties and properties of the solids in the raceways at Dogwood Lake, characterize solid settling properties, and use the results of these characterizations to design an enhanced solid removal method to increase solid settling in the quiescent zones. Velocity measurements were taken using SonTek's FlowTracker ADV in order to characterize the hydrodynamic properties of the flow through the raceway system. She took several velocity measurements throughout the entire fish zone, and quiescent zone at Dogwood Lake. These velocity measurements were sampled at a rate of $1 \mathrm{~Hz}$ and averaged over 10 second periods to derive a mean velocity for each directional component. She created three-dimensional sigma plots of the resultant velocity to describe the flow in the fish and quiescent zones (Figure 9). Figure 9 provides a great visualization of the flow in raceway B4 at Dogwood Lake, but lacks in its ability to describe the flow in detail. Rumberg did not give any detailed description of the turbulent characteristics of the flow. Also, the instrument used is sampling at are relatively slow rate, and the mean values are averaged over short time intervals. Rumberg showed that a 10 second averaging time was not adequate for capturing the accurate mean velocities in a running average plot (Figure 37).

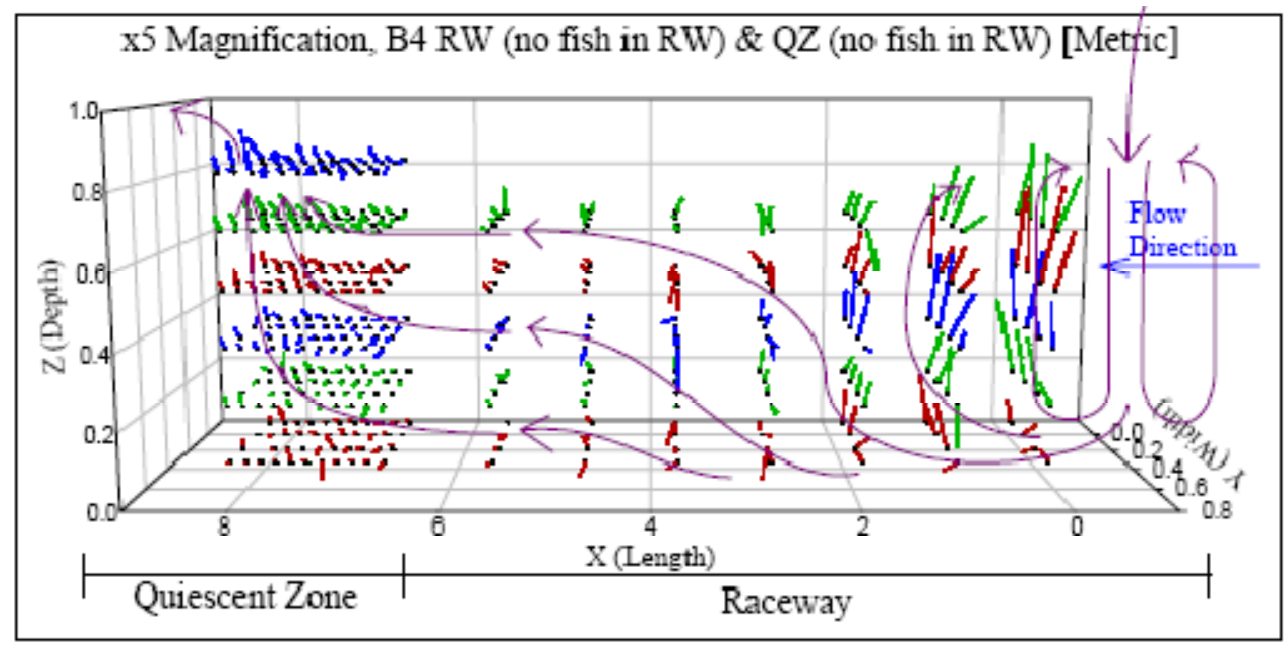

Figure 9: 3-D Plot of Resultant Velocity at Dogwood Lake (Rumberg, 2004)

Huggins (2003) used SonTek's FlowTracker ADV to measure vertical profiles of mean velocity in order to compare these to the results from a sediment transport computational fluid dynamic (CFD) model. Huggins measured velocities at a rate of $1 \mathrm{~Hz}$ and averaged the measured velocities over 30 second intervals for less turbulent areas, and 40 second intervals for 
more turbulent areas throughout the entire raceway. The raceway Huggins used to measure mean velocities has a length of $30.0 \mathrm{~m}$, a width of $3.05 \mathrm{~m}$, and quiescent zone depth of approximately $0.910 \mathrm{~m}$. The quiescent zone is partitioned by a screen and has a length of 5.33 m. Huggins was able to validate the CFD model in the quiescent zone of the raceway system. Huggins' research did not provide a detailed analysis of the flow, and its turbulent characteristics. His vertical profiles lack in detail due to the limited number of measurements and large distances between each measurement. Also, the instruments Huggins used sampled at relatively slow rates and the averaging time used to capture mean velocities is relatively low considering the Reynolds number $\left(4 \times 10^{4}\right)$ of the flow. Huggins showed that a $30-40$ second averaging time is inadequate to accurately measure mean velocities.

\subsubsection{Previous Research on Evaluating the Capability of ADVs}

\subsubsection{Direct Measurements of Reynolds Stress with an ADV}

Lohrmann et al. (1995) investigated the ability of a $10 \mathrm{Mhz}$ ADV (manufactured by SonTek) to measure Reynolds stresses (discussed in Section 4.1.2). Lormann et al. conducted an experiment in a laboratory flume comparing the turbulent fluctuations derived from the ADV to turbulent fluctuations derived from an LDV (Figure 10). They sampled at a rate of $25 \mathrm{~Hz}$ with the ADV and a rate of $50 \mathrm{~Hz}$ with the LDV over a velocity range of 5 to $80 \mathrm{~cm} / \mathrm{s}$. The result of this experiment showed a good overall agreement between the data from the two devices (Lohrmann et al., 1995). This research aids in validating the accuracy of the ADV for measuring turbulence characteristics. 


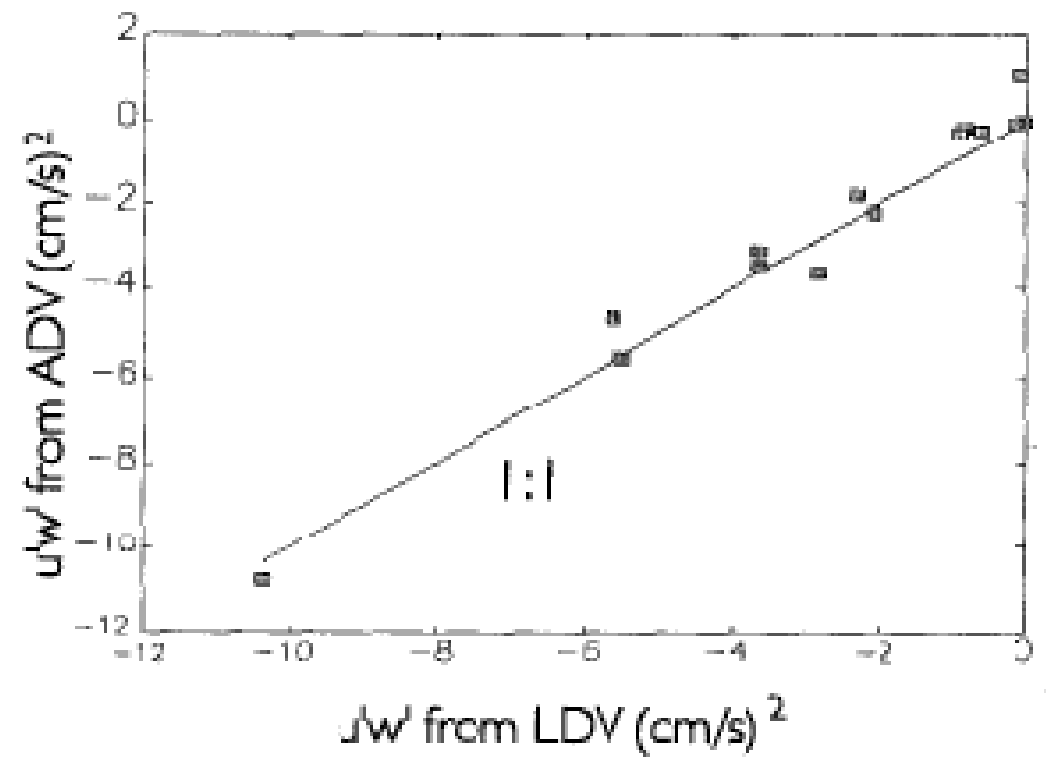

Figure 10: Turbulent Fluctuation Comparison between ADV and LDV (Lohrmann et al, 1995)

\subsubsection{Evaluation of the ADV for Turbulence Measurements}

Voulgaris and Trowbridge (1997) compared open channel turbulent flow characteristics between a Laser Doppler Velocimeter (LDV) and a $10 \mathrm{MHz}$ ADV (manufactured by SonTek). Their objective was to evaluate the ability of the ADV to measure open channel, turbulent flow characteristics. Voulgaris and Trowbridge used "ground truthing" to evaluate the performance of the ADV for measuring turbulence characteristics. "Ground truthing" involves two independent measuring methods (ADV and LDV) with known relationships between the noise and "true" flow values (Voulgaris and Trowbridge, 1997). Knowing the relationship between the noise and "true" values allows the "true" values to be determined. This method is required since an error-free device for measuring velocities does not exist (Voulgaris and Trowbridge, 1997). They conducted several experiments in a $17 \mathrm{~m}$ long, $60 \mathrm{~cm}$ wide, and $30 \mathrm{~cm}$ deep tilting flume. They conducted an experiment to determine the error of the ADV by measuring velocities in still water. They found variations in the measurements and determined an error of $\pm 0.97 \mathrm{~mm} / \mathrm{s}$ for measuring velocities at a user defined velocity range of $\pm 10 \mathrm{~cm} / \mathrm{s}$. They compared mean streamwise velocities measured at different heights and different user defined velocity ranges between the ADV and LDV. The mean streamwise velocities were derived by measuring instantaneous streamwise velocities with the LDV and ADV at a sampling rate of 25 $\mathrm{Hz}$ and an averaging time of 360 seconds (Figure 11). They also compared Reynolds stresses 
(discussed in Section 4.1.2) and squared fluctuations of vertical and transverse velocities (discussed in Section 4.1.0) derived by measurements from the ADV and LDV to the corresponding "true" values. Energy spectra were also compared by Voulgaris and Trowbridge between measurements from the ADV and LDV. Their conclusions include the following: uncertainty increases as the velocity range of the ADV increases and the ADV is capable of accurately measuring mean flow velocities, vertical turbulence intensity components, and energy spectra for both the horizontal and vertical flow components. Further, the streamwise component of the turbulence intensity is skewed from high noise levels (due to the geometry of the probe).

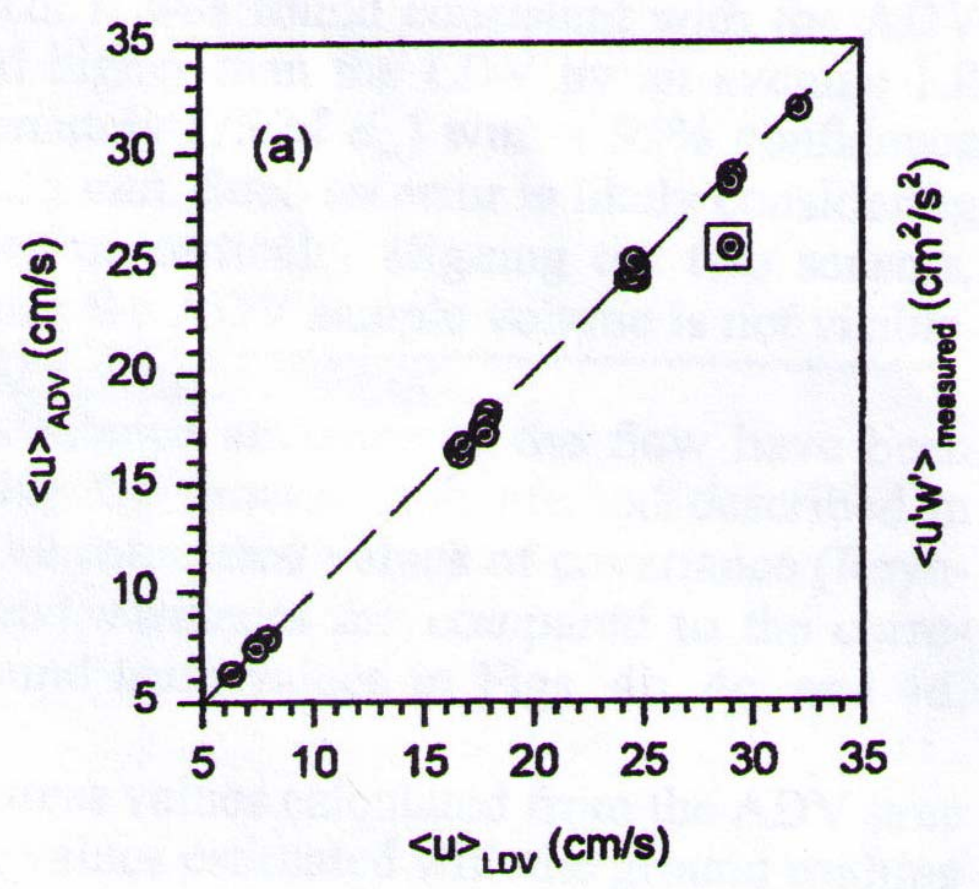

Figure 11: Mean Streamwise Velocity Comparison between ADV and LDV (Voulgaris and Trowbridge 1997)

\subsection{Background}

\subsubsection{Turbulence}

Turbulent flows consist of fluctuations in each directional component of velocity. These fluctuations are the main characteristic of turbulence. In order to obtain a single value of velocity at a point, an average of all measured values is taken over a designated time interval. This average of the velocities over a time interval is the mean velocity. The mean velocity is 
denoted by an overbar $(\bar{V})$. The velocities in each direction must be averaged separately in order to measure a value of mean velocity at a point for each directional component of velocity. Equations 1-3 show how the mean value for each component of velocity is determined,

$\bar{u}=\frac{1}{t} \int_{0}^{t} u(t) d t$

$\bar{v}=\frac{1}{t} \int_{0}^{t} v(t) d t$

$\bar{w}=\frac{1}{t} \int_{0}^{t} w(t) d t$

where $\bar{u}$ is the mean streamwise velocity, $\bar{v}$ is the mean transverse velocity, $\bar{w}$ is the mean vertical velocity, and $t$ is the averaging time.

The time interval necessary to measure an accurate mean velocity is determined by taking a running average at a point. Figure 12 shows a running average of streamwise velocities measured with the Micro ADV at $10 \mathrm{~Hz}$ at approximately $43 \mathrm{~cm}$ from the bottom of a quiescent zone at Dogwood Lake. An appropriate averaging time can be determined by finding the averaging time where the plot in Figure 12 becomes flat. From Figure 12, 200 seconds is determined as the minimum averaging time to receive an accurate mean streamwise velocity while measuring at $10 \mathrm{~Hz}$. To be conservative and allow for a possible increase of turbulence, a 360 second averaging time is used to determine mean velocities. 


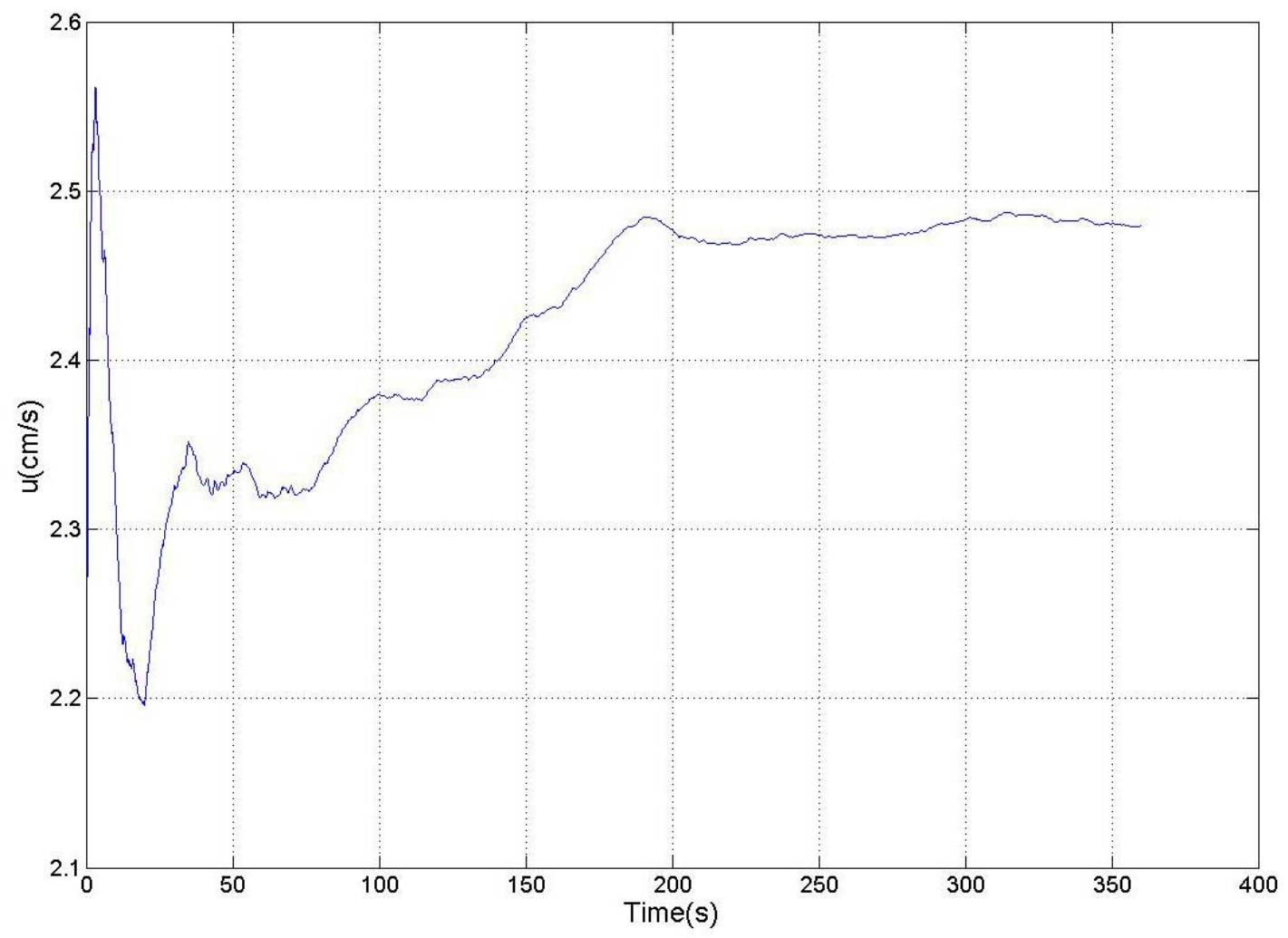

Figure 12: Running Average of Streamwise Velocity

Figure 13 shows an example of streamwise velocity fluctuations measured at Dogwood Lake. The red values are the velocities sampled at $10 \mathrm{~Hz}$ and the blue line is the mean streamwise velocity $(\bar{u})$ averaged over a 360 second time interval. 


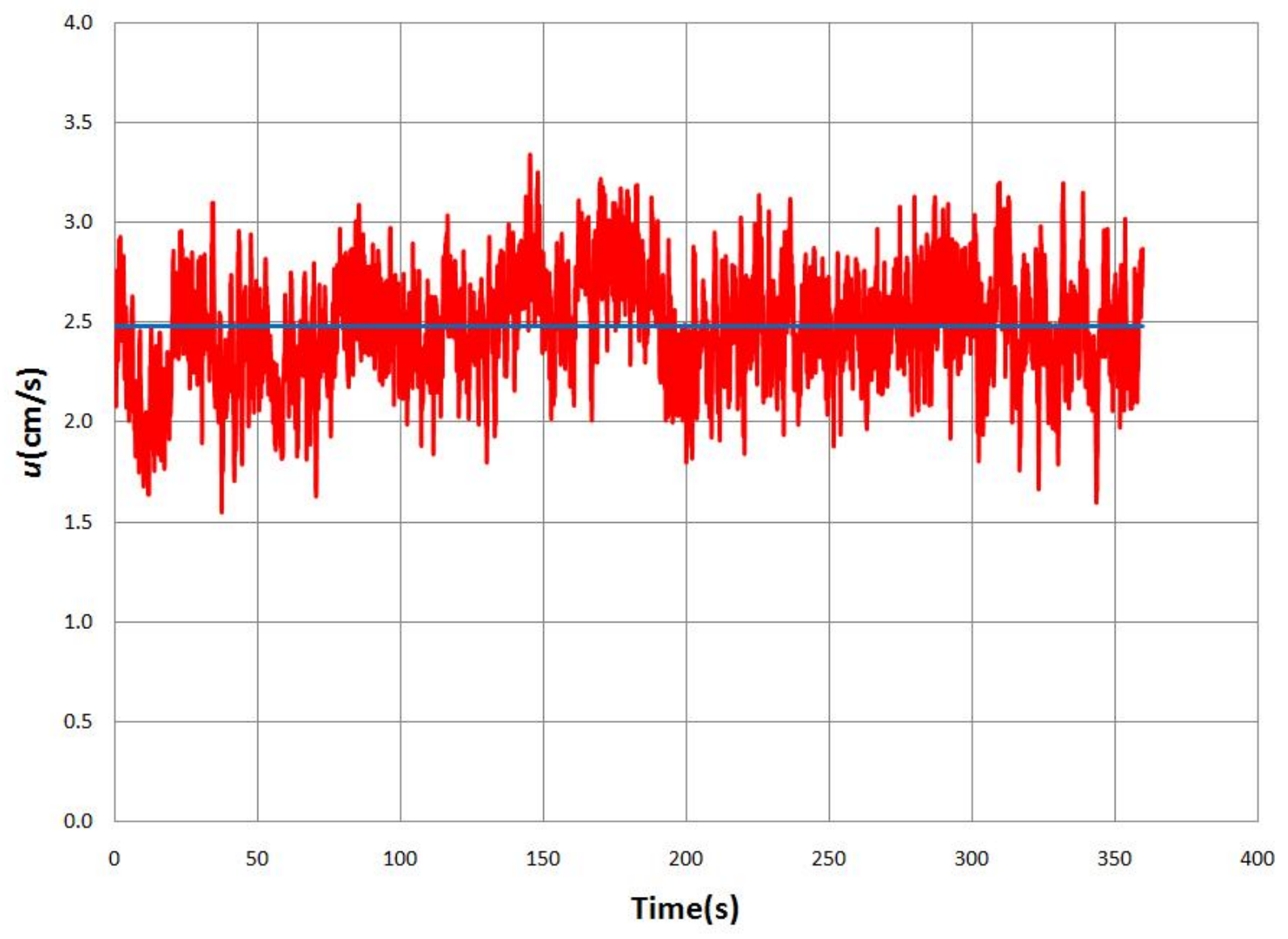

Figure 13: Streamwise Velocity Fluctuation

The intensity of the turbulence can be measured by the magnitude of the fluctuations. Equations 4-6 represent instantaneous fluctuations in each velocity component.

$$
\begin{aligned}
& u^{\prime}=u(t)-\bar{u} \\
& v^{\prime}=v(t)-\bar{v} \\
& w^{\prime}=w(t)-\bar{w}
\end{aligned}
$$

To represent the average intensity and the kinetic energy of the turbulence, the sum of the squared fluctuations must be averaged for each component of velocity (Equations 7-9).

$$
\overline{u^{\prime 2}}=\frac{1}{t} \int_{0}^{t} u^{\prime 2} d t
$$


$\overline{v^{\prime 2}}=\frac{1}{t} \int_{0}^{t} v^{\prime 2} d t$

$\overline{w^{\prime 2}}=\frac{1}{t} \int_{0}^{t} w^{\prime 2} d t$

Taking the square root of Equations 7-9 results in the root mean square (RMS) velocity for each component (Equations 10-12). The RMS is a good method for determining the average magnitude of turbulence fluctuations for each component.

$$
\begin{aligned}
& u_{R M S}=\sqrt{\overline{u^{\prime 2}}} \\
& v_{R M S}=\sqrt{\overline{v^{\prime 2}}} \\
& w_{R M S}=\sqrt{\overline{w^{\prime 2}}}
\end{aligned}
$$

The total turbulent kinetic energy (TKE) per unit mass can be found by using Equation 13 .

$$
\frac{T K E}{M a S S}=\frac{1}{2}\left(\overline{u^{\prime 2}}+\overline{v^{\prime 2}}+\overline{w^{\prime 2}}\right)
$$

The total mean turbulence intensity (TI) can be calculated using Equations 14-15 (Schlichting, 1979). The total TI and total TKE are both used to determine the total magnitude of the turbulence at a specified point in the flow.

$$
\begin{aligned}
& T I=\frac{\sqrt{\frac{1}{3}\left(\overline{u^{\prime 2}}+\overline{v^{\prime 2}}+\overline{w^{\prime 2}}\right)}}{U} \\
& U=\sqrt{\bar{u}^{2}+\bar{v}^{2}+\bar{w}^{2}}
\end{aligned}
$$




\subsubsection{Calculating Theoretical/Empirical RMS}

According to Nezu and Rodi (1986), streamwise and vertical RMS values can be approximated using Equations 16-17 in the turbulent region where the effect of viscous stresses can be neglected (discussed in 4.6.0). In Equations 16 and 17,

$$
\begin{gathered}
u_{R M S}=\sqrt{\frac{\overline{\tau_{w}}}{\rho_{w}}} D_{u} e^{\left(-\lambda_{u} \frac{z}{h}\right)} \\
w_{R M S}=\sqrt{\frac{\overline{\bar{\tau}_{w}}}{\rho_{w}}} D_{w} e^{\left(-\lambda_{w} \frac{z}{h}\right)}
\end{gathered}
$$

$\overline{\tau_{w}}$ is the mean wall shear stress, $\rho_{w}$ is the density of the water, $z$ is the distance from the bottom surface, $h$ is the flow depth, and $D_{u}, D_{w}, \lambda_{u}, \lambda_{w}$ are all empirical constants chosen to fit experimental data. Nezu and Rodi (1986) found $D_{u} \approx 2.26, D_{w} \approx 1.23, \lambda_{u} \approx 0.88$, and $\lambda_{w} \approx$ 0.67 .

\subsubsection{Reynolds Stresses}

Correlations between turbulent velocity fluctuations act as stresses called Reynolds stresses (RS). For example, consider a blob of water that fluctuates in the negative $z$-direction while maintaining its momentum in the $x$-direction. Assume the water's velocity in the $x$ direction increases in magnitude as the distance to the bottom of the raceway in the $z$-direction increases. When the blob reaches its new location, it will cause its $x$-momentum to be transferred to the new location. Since the blob moved downward where the flow in the $x$ direction is slower, there is an increase in the $x$-momentum at the new location $\left(\rho_{w} u{ }^{\prime}\right)$. The rate at which momentum is gained in the $x$-direction is $\rho_{w} u^{\prime} v^{\prime}$, where $\rho_{w}$ is the density of water. The rate at which momentum is transferred is considered a force; thus $\rho_{w} u^{\prime} v^{\prime}$ can be measured as a stress due to correlated fluctuations (Gray, 1982). This allows a mean stress due to correlated turbulent fluctuations (RS) to be obtained by taking the mean of the product of correlated velocity fluctuations (Equations 18-23).

$$
R S_{x z}=R S_{z x}=-\rho_{w} \overline{u^{\prime} w^{\prime}}
$$




$$
\begin{aligned}
& R S_{x y}=R S_{y x}=-\rho_{w} \overline{u^{\prime} v^{\prime}} \\
& R S_{y z}=R S_{z y}=-\rho_{w} \overline{v^{\prime} w^{\prime}} \\
& R S_{x x}=-\rho_{w} \overline{u^{\prime} u^{\prime}} \\
& R S_{y y}=-\rho_{w} \overline{v^{\prime} v^{\prime}} \\
& R S_{z z}=-\rho_{w} \overline{w^{\prime} w^{\prime}}
\end{aligned}
$$

\subsubsection{Two-Dimensional Fully Developed Turbulent Open Channel Flow}

A description of two-dimensional, fully developed, open channel, turbulent flow is necessary in understanding the background of this research. Characteristics measured by the ADV are compared to this type of flow due to the existence of theoretical/empirical calculations that define its characteristics. Assuming this type of flow enables the Reynolds stress terms to be theoretically/empirically calculated. Without these assumptions the velocity profiles could not be theoretically/empirically calculated.

\subsubsection{Fully Developed Flow}

Fully developed flow is defined by flow characteristics that remain constant as the flow moves downstream. As water enters an open channel, the viscous forces on the water from the boundaries and forces caused by fluctuations in the flow from the turbulence begin to affect the flow. When the effects of the two forces become constant in the streamwise direction, the flow is fully developed.

The length of the channel must be long enough without any disturbances present for the flow to contain fully developed characteristics. Trowbridge et al. (1989) suggests that the length of the channel should be 50-100 times the depth of the water in order for the flow to achieve fully developed characteristics. In addition, the flow through the channel should be uniform in depth. Uniformity in flow depth represents a loss of potential energy equal to the loss of energy due to viscous forces. When this occurs, the flow depth will remain constant down the slope of the channel. In order to assume uniform flow depth, the fluid must flow through a uniform cross-sectional size and shape (Young et al., 2004). 


\subsubsection{Two-Dimensional Flow}

Two-dimensional flow is described using two dimensions. For open channels which contain two-dimensional flow, the flow near the center of the channel $(W / 2)$ can be described using the vertical $(z)$ and streamwise $(x)$ directions. This is because two-dimensional flow near the center of the channel varies in the vertical direction $(z)$ as well as the streamwise direction $(x)$. Two-dimensional flow varies with respect to the transverse direction $(y)$ near the side walls of an open channel; however, there is a region near the center where no change in the flow with respect to the transverse direction exists. The change in flow is due to viscous forces on the water from the walls and bottom surface of the channel. Near any boundary, the flow will be retarded due to viscous forces. As the distance from a boundary increases, the effects that viscous forces have on the flow will decrease.

A wide channel is required for viscous forces near the center to become zero. In order to achieve two-dimensional flow characteristics near the center of an open channel, it is suggested by Nakagawa et al. (1983) that the aspect ratio (width-to-depth ratio) of the channel should be greater than 6.

\subsubsection{Dimensionless Parameters}

\subsubsection{Reynolds Number}

The Reynolds number is an important property when considering open channel flow. The Reynolds number for open channel flow is shown in Equation 24,

$R e=\frac{4 A \bar{V}}{v_{w} P_{w}}$

where $A$ represents the cross sectional area of the flow, $\bar{V}$ is the average velocity over the entire area, $P_{w}$ is the wetted perimeter of the channels cross section, and $v_{w}$ is the kinematic viscosity of water. The Reynolds number describes the ratio of inertial forces to viscous forces, making it a dimensionless parameter. A large Reynolds number describes a flow where the inertial forces outweigh the viscous forces. In open channel flow, a Reynolds number $>8000$ is considered turbulent (Gray, 2008). In turbulent flows, the flow in the thin layer called the viscous sub-layer 
(better described in Section 4.5.0) is determined mostly by viscous forces. As the distance from the bottom increases, the flow becomes independent of viscosity. The viscous force at a wall causes a no slip condition, meaning there is no flow at the wall. Moving away from the wall, the viscous forces retard the flow less and less until the effect of viscosity is no longer present. The Reynolds number can help determine the magnitude of the turbulence.

\subsubsection{Froude Number}

The Froude number is also a dimensionless parameter, but describes the ratio of inertial forces to body forces. If the Froude number is less than one, the flow is subcritical. If greater than one, it is supercritical. When the main flow is subcritical, the body forces outweigh the inertial forces and disturbances will have an effect on the flow upstream as well as downstream. When the flow is supercritical, the inertial forces outweigh the body forces and disturbances are carried downstream and do not have an effect on the upstream flow. Equation 25 is used to calculate values for the Froude number,

$$
F r=\frac{\overline{\bar{V}}}{\sqrt{h g}}
$$

where $\bar{V}$ is the depth average velocity, $h$ is the depth of the water, and $g$ is the acceleration due to gravity.

\subsubsection{Mean Stress Distribution}

The control volume in Figure 14 is used to derive the total mean stress distribution. Assume the flow in the control volume contains mean steady, two-dimensional, fully developed, open channel turbulent flow. Since the flow is mean steady, the acceleration of the control volume is zero. Applying Newton's second law of motion per unit area in the $x$-direction

( $\sum \frac{\text { Forces }}{\text { Area }}=\frac{m a}{\text { Area }}$, where $m$ is mass, and $a$ is acceleration) results in the sum of the forces per unit area equal to zero.

The forces acting on the control volume in the $x$-direction are: the hydrostatic pressure forces $\left(F_{1}, F_{2}\right)$, the total mean shear stress forces $\left(F_{v x}\right.$, Equation 26), and the streamwise component gravitational forces $\left(F_{g x}\right.$, Equation 27$)$. 


$$
\begin{aligned}
& \bar{\tau}=\frac{F_{v x}}{\text { Area }}=\mu_{w} \frac{\partial \bar{u}}{\partial z}-\rho_{w} \overline{u^{\prime} w^{\prime}} \\
& \frac{F_{g x}}{\text { Area }}=\rho_{w} g(h-z) \sin (\theta)
\end{aligned}
$$

Because the flow possesses uniform flow depth, the hydrostatic pressure forces cancel out. Equation 28 represents Newton's second law per unit area.

$$
\frac{F_{g x}}{\text { Area }}-\bar{\tau}=0
$$

Solving Equation 28 for the total shear stress $(\bar{\tau})$ results in Equation 29.

$$
\bar{\tau}=\rho_{w} g \sin (\theta)(h-z)
$$

Equation 29 shows that as $z$ approaches $h$, the total shear stress varies linearly (Trowbridge et al., 1989). Using Equation 29 at the bottom of the raceway $(z=0)$, one can find the wall shear stress (Equation 30).

$$
\overline{\tau_{w}}=\rho_{w} g h \sin (\theta)
$$

Since viscous shear stresses $\left(\mu_{w} \frac{\partial \bar{u}}{\partial z}\right)$ become negligible outside the viscous sub-layer, the Reynolds stress due to turbulent fluctuations can be found by eliminating the shear stress term in Equation 26. 


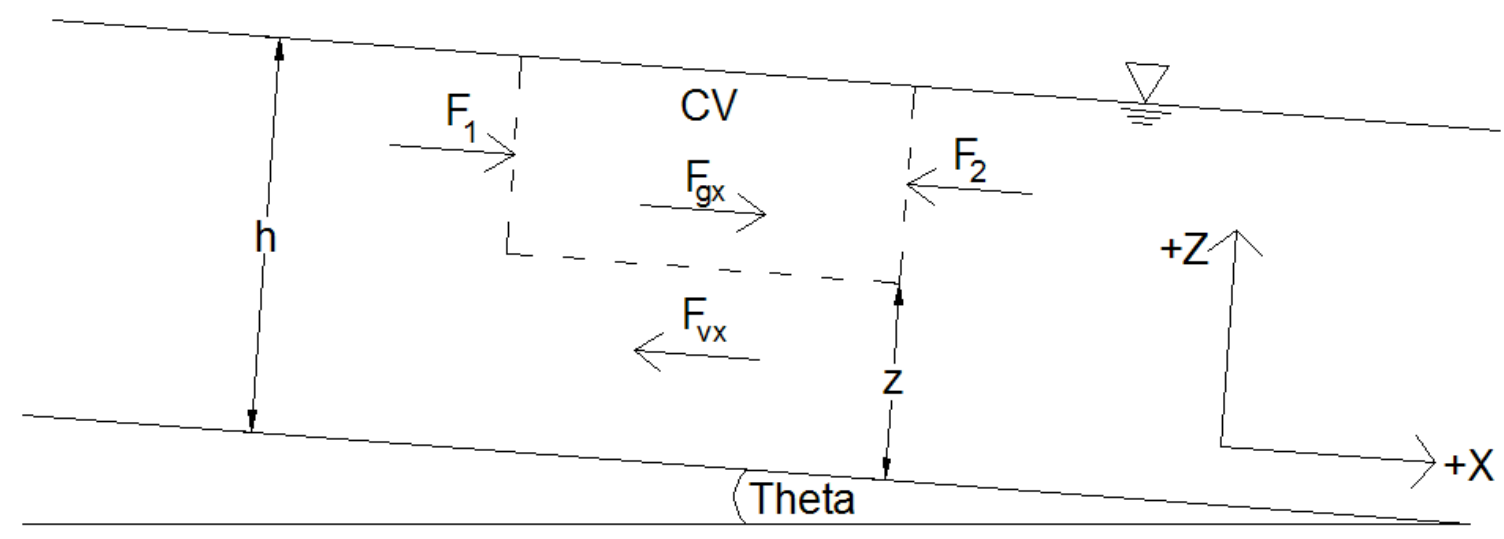

Figure 14: Forces Acting on a Control Volume

\subsubsection{Mean Velocity Distribution}

The flow is considered to have two-dimensional, fully developed, open channel turbulent flow near the center line. As mentioned in Section 4.2.0, the flow is assumed to have the previously mentioned characteristics. These characteristics are assumed since many experiments have been performed in open channels, allowing theoretical/empirical equations to exist. From the results of the measured profiles, this assumption is not accurate. The actual flow through the raceway system is not fully developed or two-dimensional; however, this assumption is used to show how the theoretical/empirical equations are derived.

Two-dimensional, fully developed, open channel turbulent flow represents a change in $\bar{u}$ with respect to the $z$-direction only. Also, both mean velocity components normal to $\bar{u}$ are zero $(\bar{u}(z) \neq 0, \bar{v}=\bar{w}=0)$. When approaching the side walls of the channel, $\bar{u}$ will vary due to the viscous wall shear stress.

For flow variation in the $z$-direction near the center line, one can approximate the flow by considering steady, incompressible flow along an infinitely long plate where the only pressure gradient that exists is in the $x$-direction and it is constant ( $\frac{\partial \bar{P}}{\partial x}=$ Constant). This causes $\bar{u}$ to vary in the vertical (z-direction) only, and the mean components of velocity normal to $\bar{u}$ to become zero $(\bar{v}=\bar{w}=0)$. The variation in the $z$-direction exhibits three regions or layers: the viscous sub-layer, the buffer layer, and the turbulent layer (Yuan, 1967). The viscous sub-layer is a thin section close to the wall where viscous stresses outweigh Reynolds stresses. The buffer 
layer is located between the viscous sub-layer and the turbulent layer. In the buffer layer, viscous and Reynolds stresses are of the same order of magnitude. The turbulent layer lies above the buffer layer and is where Reynolds stresses are dominant over viscous stresses. The mean velocity distribution in the viscous sub-layer $\left(\frac{u_{*} z}{v_{w}}<5\right)$ can be approximated by Equations 31-32,

$$
\begin{gathered}
u_{*}^{2}=\frac{\overline{\tau_{w}}}{\rho_{w}} \\
\bar{u}=\frac{u_{*}{ }^{2} z}{v_{w}}
\end{gathered}
$$

where $u_{*}$ is the friction velocity, and $v_{w}$ is the kinematic viscosity of the water. The mean distribution in the turbulent layer $\left(\frac{u_{*} Z}{v_{w}}>70\right)$ can be approximated using Equation 33,

$\bar{u}=\left(\frac{u_{*}}{k}\right) \ln \frac{u_{*}}{v_{w}} z-\left(u_{*}\right) \ln \beta$

where $k$ is the non-dimensional von Karman constant, and $\beta$ is another non-dimensional constant. The two constants in Equation 33 are determined empirically. Experimental results from Nikuradse and Reichardt (1945) yield values of 0.40 and 0.11 for $k$ and $\beta$, respectively. Von Karman suggested the buffer layer $\left(5<\frac{u_{*} z}{v_{w}}<70\right)$ is approximated by Equation 33 with values of 0.2 and 21.11 for $k$ and $\beta$, respectively (Yuan, 1967). Nezu and Rodi (1986) suggested using Coles wake function for correcting any discrepancies from the log-law. However, the Reynolds number $\left(2 \times 10^{4}\right)$ at Dogwood Lake is relatively small, therefore Coles wake function is not necessary and Equation 33 can be used for approximating $\bar{u}$ in the turbulent layer.

In order to approximate the change in $\bar{u}$ in the transverse direction due to the shear stress of both walls, one can consider steady, incompressible flow between two infinitely long parallel plates. Again, the only pressure gradient that exists is in the $x$-direction and is considered constant $\left(\frac{\partial \bar{P}}{\partial x}=\right.$ Constant $)$. The same three layers exist at both walls; however, an approximation must be made from both walls to the center $(\mathrm{W} / 2)$ so that the effects of both walls are accounted for. This is done by creating a velocity defect law (Equation 34) and applying 
Equation 33 at the center line $(\mathrm{W} / 2)$ and subtracting Equation 33 evaluated at an unspecified distance from the wall (Gray, 1982). In Equation 34,

$\frac{\bar{u}_{\max }-\bar{u}}{u_{*}}=\frac{1}{k} \ln \left(\frac{W}{2 y}\right)$

$\bar{u}_{\text {max }}$ is the maximum velocity at the center line and $W$ is the width of the channel. Applying Equation 34 will create an unrealistic cusp in the center of the channel showing that viscous forces are present. There should not be viscous forces present in the center of the channel and the cusp should be ignored.

\subsubsection{Energy Spectrum}

Consider turbulent flow as a cluster of eddies with different length scales. The larger eddies contain larger amounts of energy and the smaller eddies contain smaller amounts of energy. Kolmogorov found a relationship between the large and small eddies by simply considering an energy budget (see Bradshaw, 1971 and Frisch, 1995). A large eddy containing large amounts of energy transfers its energy to the smaller ones. The smaller eddies are then dissipated by viscous stresses and the energy from these eddies is transformed into heat energy. Determining the correlation between fluctuations at two points separated by vector $\boldsymbol{r}=r_{1} i+$ $r_{2} j+r_{3} k$ can determine the size of eddy and thus the amount of energy it contains. Equations 35-37 show the spatial correlation functions with respect to each flow direction.

$$
\begin{aligned}
& R D_{11}(\boldsymbol{r})=\overline{u^{\prime}(x, y, z, t) u^{\prime}\left(x+r_{1}, y+r_{2}, z+r_{3}, t\right)} \\
& R D_{22}(\boldsymbol{r})=\overline{v^{\prime}(x, y, z, t) v^{\prime}\left(x+r_{1}, y+r_{2}, z+r_{3}, t\right)} \\
& R D_{33}(\boldsymbol{r})=\overline{w^{\prime}(x, y, z, t) w^{\prime}\left(x+r_{1}, y+r_{2}, z+r_{3}, t\right)}
\end{aligned}
$$


Taking the Fourier transform of the correlation functions results in the wave-number spectrums in each direction (Equations 38-40, where $k_{1} k_{2}$, and $k_{3}$ are wave-numbers corresponding to $r_{1}, r_{2}$, and $r_{3}$ ).

$$
\begin{aligned}
& \Phi S_{11}\left(k_{1}\right)=\frac{1}{\pi} \int_{-\infty}^{\infty} R D_{11}(\boldsymbol{r}) e^{-i k_{1} r_{1}} d \boldsymbol{r} \\
& \Phi S_{22}\left(k_{2}\right)=\frac{1}{\pi} \int_{-\infty}^{\infty} R D_{22}(\boldsymbol{r}) e^{-i k_{2} r_{2}} d \boldsymbol{r} \\
& \Phi S_{11}\left(k_{3}\right)=\frac{1}{\pi} \int_{-\infty}^{\infty} R D_{33}(\boldsymbol{r}) e^{-i k_{3} r_{3}} d \boldsymbol{r}
\end{aligned}
$$

Considering that the spatial correlation is difficult to measure experimentally, a temporal correlation must be found. Taylor's hypothesis of frozen turbulence states that if the fluctuations in the flow are much smaller than the mean velocity, the rate of change of the eddy can be neglected as it passes the measurement point. Sampling at a point over time allows a variety of eddies to pass the sampling point. This enables temporal correlations to be found (Equations 4143 , where $P_{t}$ is the time delay between fluctuations).

$$
\begin{aligned}
& R T_{11}\left(P_{t}\right)=\overline{u^{\prime}(x, y, z, t) u^{\prime}\left(x, y, z, t+P_{t}\right)} \\
& R T_{22}\left(P_{t}\right)=\overline{v^{\prime}(x, y, z, t) v^{\prime}\left(x, y, z, t+P_{t}\right)} \\
& R T_{33}\left(P_{t}\right)=\overline{w^{\prime}(x, y, z, t) w^{\prime}\left(x, y, z, t+P_{t}\right)}
\end{aligned}
$$

Taylor's hypothesis at Dogwood Lake is valid. When looking at a single measuring point at Dogwood Lake, it is found that the mean streamwise velocity at this point is approximately 12 times the size of the average magnitude of fluctuations in the streamwise direction. This allows for the change in structure of an eddy passing the sampling point to be ignored. 
Taking the Fourier transform of the temporal correlations will result in the frequency spectra (Equations 44-46, where $f$ is the frequency).

$$
\begin{aligned}
& \Phi t_{11}(f)=\frac{1}{\pi} \int_{-\infty}^{\infty} R T_{11}\left(P_{t}\right) e^{-i f P_{t}} d P_{t} \\
& \Phi t_{22}(f)=\frac{1}{\pi} \int_{-\infty}^{\infty} R T_{22}\left(P_{t}\right) e^{-i f P} d P_{t} \\
& \Phi t_{33}(f)=\frac{1}{\pi} \int_{-\infty}^{\infty} R T_{33}\left(P_{t}\right) e^{-i f P} d P_{t}
\end{aligned}
$$

Within the energy spectrum, the range of wave-numbers or frequencies where the large eddies transfer their energy to the smaller eddies is considered the inertial sub-range. Kolmogorov found that in turbulent flow with large Reynolds numbers Equation 47 can be used to describe the wave-number energy spectrum.

$$
\Phi(k)=\alpha \varepsilon^{\frac{2}{3}} k^{-\frac{5}{3}}
$$

Equation 47 shows the variation of the wave-number spectrum within the inertial sub-range with respect to the wave-number, turbulent energy dissipation ( $\varepsilon$, rate that small eddy energy is transformed into heat energy), and empirical constant $(\alpha)$.

\subsubsection{Acoustic Doppler Velocimeter}

The ADV is a good instrument for measuring water velocities in field environments. It is a fairly rugged instrument that requires simple setup when compared to other instruments (Laser Doppler Velocimeter (LDV), Particle Tracking Instruments (PTI)), no calibration, can sample at relatively high frequencies (allowing turbulence to be measured), and is cost effective. Also, the ADV does not create a disturbance where the velocities are measured, as a hot wire anemometer or pitot tube would. 
The ADV operates by means of the Doppler frequency shift principle in order to measure three-dimensional instantaneous velocities. The transmitter generates a narrow sound wave pulse train with the majority of its energy concentrated in a narrow cone (SonTek, 2001). The pulses pass through a sampling volume and are reflected off particles in the water. The receivers then measure the reflected pulses. The change in frequency between the known signal and the received signal enables the velocity to be measured (Equation 48). In Equation 48,

$V=\left(\frac{\Delta f}{f_{\text {Source }}}\right) C$

$C$ is the speed of the sound wave, $f_{\text {Source }}$ is the frequency of the transmitted sound wave, $\Delta f$ is the change in frequency with respect to the source frequency, and $V$ is the measured velocity.

\subsection{Materials and Method}

\subsubsection{SonTek’s Micro ADV}

Velocity measurements at Dogwood Lake were taken using SonTek's $16 \mathrm{MHz}$ Micro ADV. This instrument is capable of measuring velocities in three directions. The ADV probe consists of an underwater connector, signal conditioning module, a stem, and acoustic sensors. A waterproof cable connects the ADV probe to a processing module and a serial cable connects the processing module to a laptop where the data is viewed and stored. The total length of the probe from the underwater connector to the acoustic sensors is approximately $57 \mathrm{~cm}$. The diameter of the signal conditioning module is approximately $5 \mathrm{~cm}$. The acoustic sensors measure the velocities using a bistatic axis orientation. The acoustic sensor is comprised of a transmitter surrounded by three receivers. The receivers are separated by 120 degrees and are angled inward 30 degrees with respect to the plane of the transmitter. 


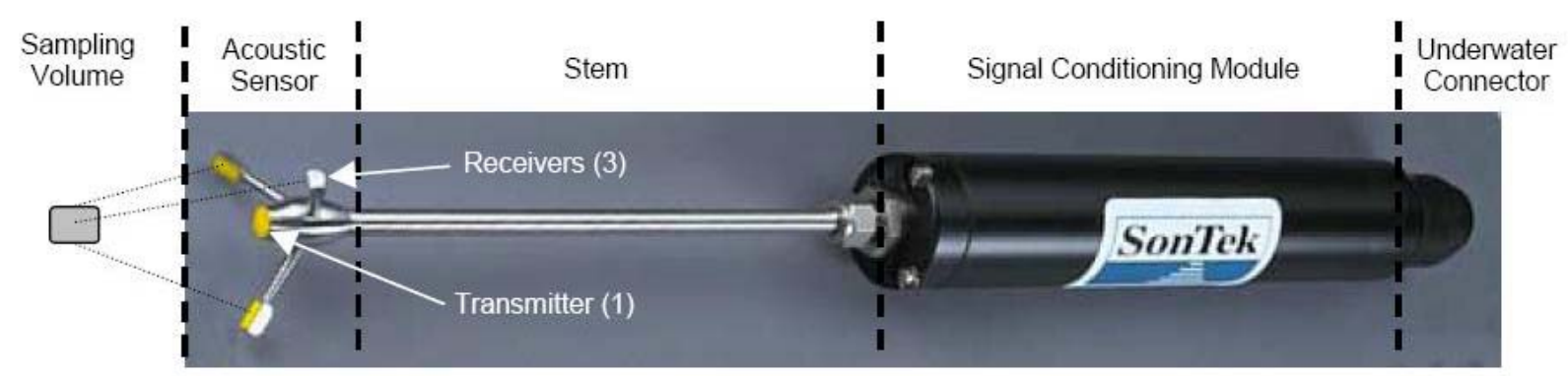

Figure 14: SonTek's 16 MHz Micro ADV (Picture from SonTek Manual)

*Please note that the sampling volume is enlarged.

This ADV samples at a user defined frequency between 0.1-50 samples per second (Hz), has user defined velocity range (Table 2), a resolution of $0.01 \mathrm{~cm} / \mathrm{s}$, and an accuracy of $1 \%$ of measured velocities (SonTek, 2001).

Table 2: Micro ADV Velocity Ranges

\begin{tabular}{|c|c|}
\hline Range Number & Velocity Range (cm/s) \\
\hline 1 & \pm 3 \\
\hline 2 & \pm 10 \\
\hline 3 & \pm 30 \\
\hline 4 & \pm 100 \\
\hline 5 & \pm 250 \\
\hline
\end{tabular}

The sampling rate is chosen by the user, but there is an internal sampling rate determined by the environment in which the ADV is measuring. This internal sampling rate is called the ping rate. A ping is a single estimate of velocity (SonTek, 2001) and varies depending on the amount of particles in the water. For example, if the sampling rate is at $1 \mathrm{~Hz}$, there will be 1 measured velocity outputted every second. This velocity is determined by the number of pings that are measured within the 1 second interval. The Micro ADV pings at rate of 150-250 pings per second. Even if the user set sampling rate is at $1 \mathrm{~Hz}$, there are 150-250 pings averaged to output 1 velocity measurement every second.

The sampling volume for the Micro ADV (approximately $0.25 \mathrm{~cm}^{3}$ ) is located approximately $5 \mathrm{~cm}$ from the transmitter. The size of the sampling volume is determined by the 
pattern of the transmitted beam, the pattern of the reflected beam, the length of each transmitted pulse, and the period of time over which the return signal is sampled (SonTek, 2001). Variables within the ADV software may be changed in order to modify the dimensions of the sampling volume, but for this research it is considered constant. The sampling volume for the Micro ADV is cylindrical in shape with a diameter of approximately $6 \mathrm{~mm}$ and a height of $9 \mathrm{~mm}$.

\subsubsection{Receiving Accurate Measurements from the Micro ADV}

In order for the ADV to perform properly, measurement parameters suggested by the manufacture of the ADV (SonTek) and Garcia et al. (2004) should be considered.

Several performance parameters are directly outputted from the Micro ADV for each measured velocity. The performance parameters allow for a check to see if the environment is suitable for the ADV to accurately measure velocities. The correlation value that is outputted for each measurement allows the user to determine the percentage of correlation between pings (discussed in Section 5.1.0). If the pings are correlated, than the correlation value for that measurement will be high, representing an accurate measurement. If the pings are not correlated, the value will be low, representing high turbulence levels and/or high noise levels. Since the Reynolds number is moderately low (representing moderate turbulence) at Dogwood Lake and the ping rate is high, a low correlation at Dogwood Lake is most likely related to a high noise levels. Correlation values are expressed as a percentage (SonTek, 2001). It is suggested that correlation values be between 70 and 100\% (SonTek, 2001).

Another directly outputted parameter is the Signal-to-Noise Ratio (SNR). The SNR is determined by the strength of the received pulses. The SNR can be related to the amount of particles in the water that are reflecting the transmitted pulse back to the receivers. If the single strength is low, there may be a lack of particles and reflections measured are most likely reflections that did not take place in the sampling volume (noise). SonTek (2001) suggests that the SNR be greater than $5 \mathrm{~dB}$ in order to receive accurate mean velocity measurements.

Another factor in receiving accurate measurements is selecting the proper velocity range (Table 2). If a large velocity range is selected, the transmitter transmits pulses with less lag time between each pulse even if the sampling rate is unchanged. The more pulses that are transmitted, the larger the potential is for noise to affect the measurements. SonTek suggests choosing the lowest range that will encompass the maximum velocity expected in the flow (SonTek, 2001). 
Selecting an appropriate frequency for measuring three-dimensional velocities is also important for receiving accurate results. A larger sampling rate will result in more noise associated with measured velocity. SonTek (2001) suggests reducing the sampling rate as much as possible in order to eliminate unwanted noise by averaging more pings.

Garcia et al. (2004) suggest calculating an F factor in order to determine how well the ADV will measure turbulence. The larger the $\mathrm{F}$ factor is, the fewer small scale fluctuations the $\mathrm{ADV}$ will ignore. Equation 49 represents the $\mathrm{F}$ factor,

$$
F=\frac{L}{d_{R}}
$$

where $\mathrm{L}$ is the largest possible eddy that will occur in the flow and $d_{R}$ is the diameter of the sampling volume. Garcia et al. conclude that an $\mathrm{F}$ factor greater than 20 is adequate for receiving accurate turbulence measurements. Assuming a constant control volume, the $\mathrm{F}$ factor (Equation 49) is approximated to be 148 , using the width of the quiescent zone $(88.9 \mathrm{~cm})$ as the largest possible eddy and the diameter of the sampling volume of $6 \mathrm{~mm}$. A value of 148 is more than sufficient to provide accurate turbulence measurements according to Garcia et al. (2004).

\subsubsection{ADV Uncertainty}

All Doppler systems have measurement noise. This noise is a result of scattered sound waves that may be reflecting off objects outside of the sampling volume, thus causing error in the measured velocities (Doppler noise). Due to the probe alignment, the Micro ADV has different errors for the directional components of measured velocity. Since the transmitter is on the vertical axis, the acoustic receivers are more sensitive to measuring vertical velocities than streamwise and transverse velocities by a factor of 4 . The noise associated with measuring streamwise and transverse velocities is estimated to be $1 \%$ of the user defined velocity range when sampling at $25 \mathrm{~Hz}$ (SonTek, 2001). For example, sampling at $25 \mathrm{~Hz}$ with a velocity range of $\pm 10 \mathrm{~cm} / \mathrm{s}$ will create an error of $\pm 0.10 \mathrm{~cm} / \mathrm{s}$. However, the noise level decreases with the square root of the number of pings averaged per recorded velocity (SonTek, 2001). If the sampling rate is changed to $10 \mathrm{~Hz}$ (sampling rate used throughout this research) and averaged over a 360 second interval (time interval used throughout this research) the reduction in error is approximately $73(\sqrt{15 \times 360})$ times less than sampling at $25 \mathrm{~Hz}$ for 1 second. This results in an 
error of approximately $\pm 1.4 \times 10^{-3} \mathrm{~cm} / \mathrm{s}$. This error for the mean vertical velocity is 4 times less than the transverse and streamwise $\left( \pm 3.4 \times 10^{-4} \mathrm{~cm} / \mathrm{s}\right)$. Placing theses errors into the equations for the other measured characteristics allows for the errors associated with all measured flow characteristics to be found (Table 3). These errors are very small and can be ignored for all mean measurements.

Table 3: Error in Measured Characteristics Due to ADV Uncertainty

\begin{tabular}{|c|c|c|c|c|c|c|c|c|}
\hline $\begin{array}{c}\bar{u} \\
(\mathrm{~cm} / \mathrm{s})\end{array}$ & $\begin{array}{c}\bar{v} \\
(\mathrm{~cm} / \mathrm{s})\end{array}$ & $\begin{array}{c}\bar{w} \\
(\mathrm{~cm} / \mathrm{s})\end{array}$ & $\begin{array}{c}\overline{u^{\prime 2}} \\
\left(\mathrm{~cm}^{2} / \mathrm{s}^{2}\right)\end{array}$ & $\begin{array}{c}\overline{v^{\prime 2}} \\
\left(\mathrm{~cm}^{2} / \mathrm{s}^{2}\right)\end{array}$ & $\begin{array}{c}\overline{w^{\prime 2}} \\
\left(\mathrm{~cm}^{2} / \mathrm{s}^{2}\right)\end{array}$ & $\begin{array}{c}\overline{u^{\prime} w^{\prime}} \\
\left(\mathrm{cm}^{2} / \mathrm{s}^{2}\right)\end{array}$ & $\begin{array}{c}\overline{u^{\prime} v^{\prime}} \\
\left(\mathrm{cm}^{2} / \mathrm{s}^{2}\right)\end{array}$ & $\begin{array}{c}\overline{v^{\prime} w^{\prime}} \\
\left(\mathrm{cm}^{2} / \mathrm{s}^{2}\right)\end{array}$ \\
\hline $\pm 1.4 \mathrm{E}-03$ & $\pm 1.4 \mathrm{E}-03$ & $\pm 3.4 \mathrm{E}-04$ & $\pm 2.0 \mathrm{E}-06$ & $\pm 2.0 \mathrm{E}-06$ & $\pm 1.2 \mathrm{E}-07$ & $\pm 4.8 \mathrm{E}-07$ & $\pm 2.0 \mathrm{E}-06$ & $\pm 4.8 \mathrm{E}-07$ \\
\hline
\end{tabular}

\subsubsection{Traversing Mechanism}

In order for the ADV to traverse throughout the quiescent zone and remain stationary while taking measurements, a traversing mechanism has been constructed. The traversing mechanism consists of a bridge (Figure 15) that lies across the quiescent zone, a cart that is supported by the bridge (Figure 16), and a hollow aluminum beam (Figure 17) that supports the ADV while connected to the cart.

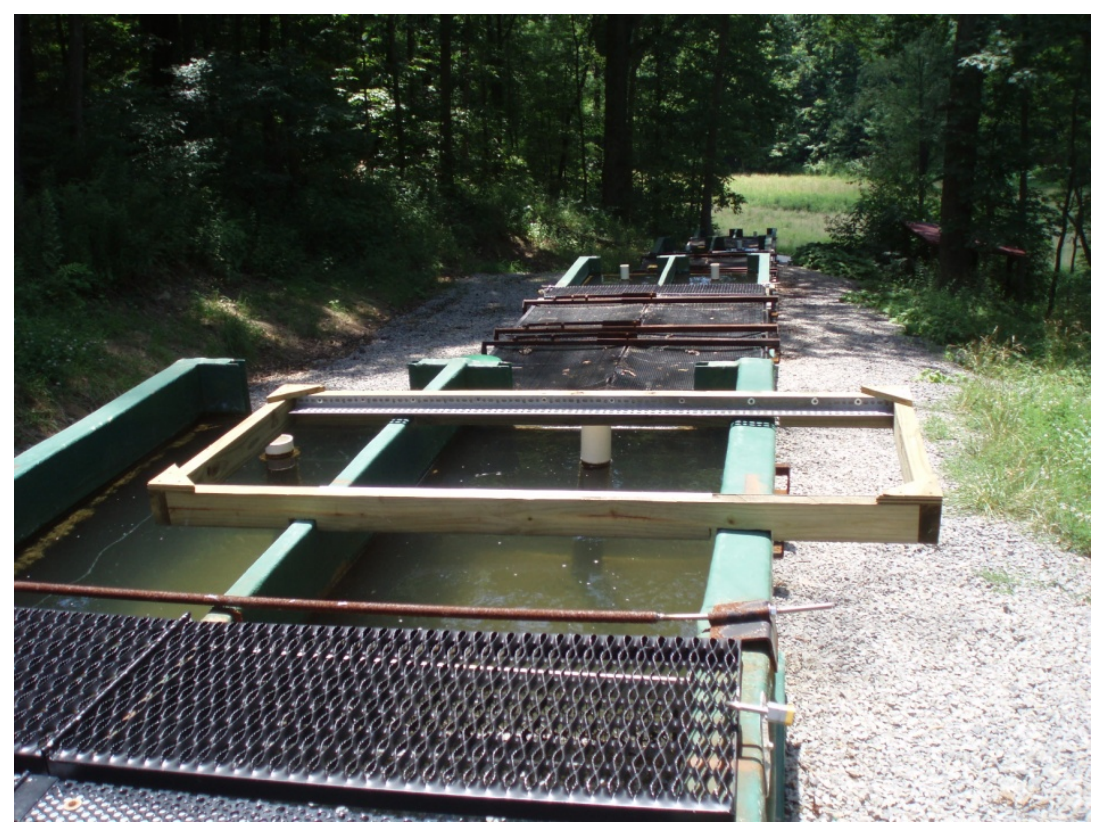

Figure 15: Traversing Mechanism Bridge 


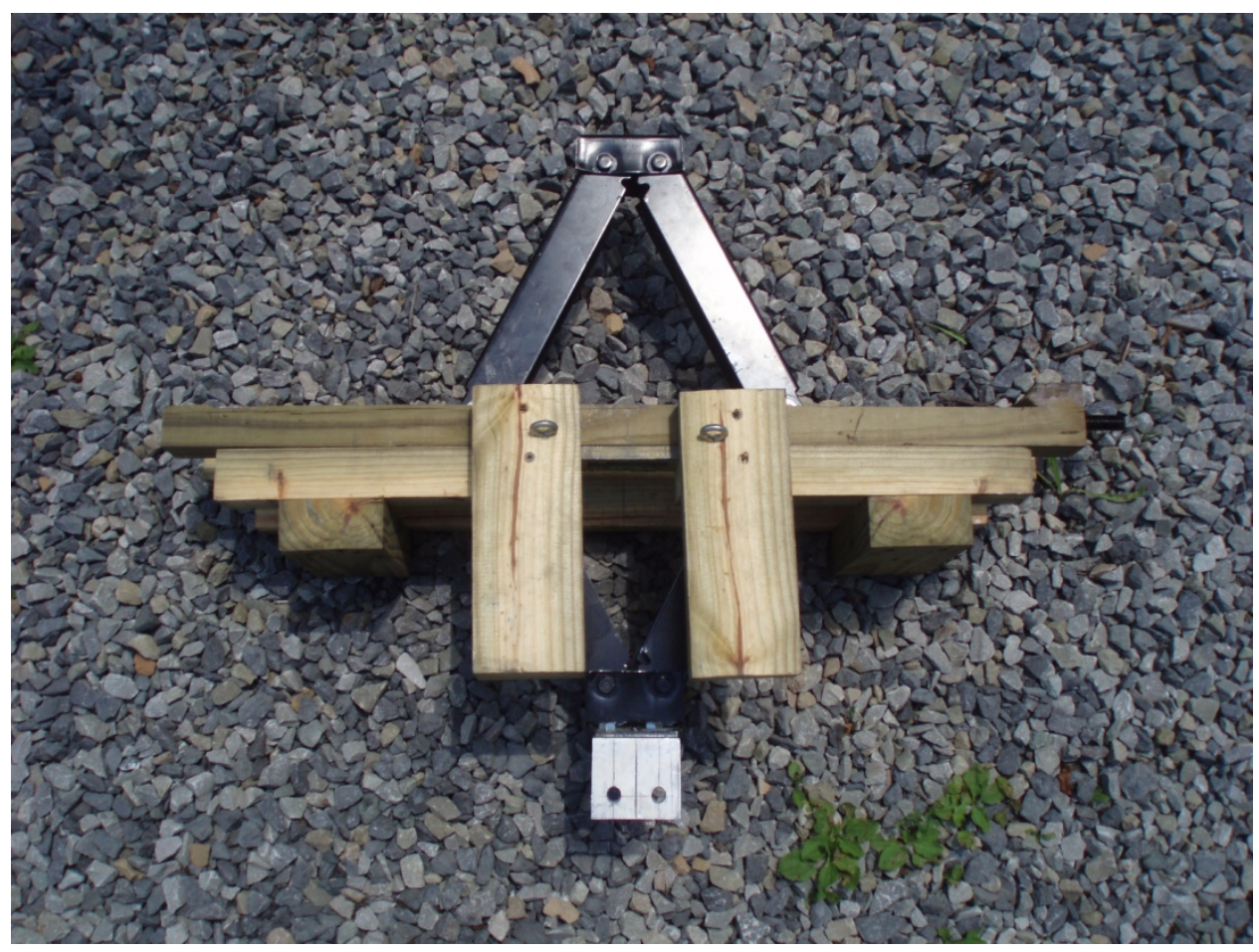

Figure 16: Traversing Mechanism Cart

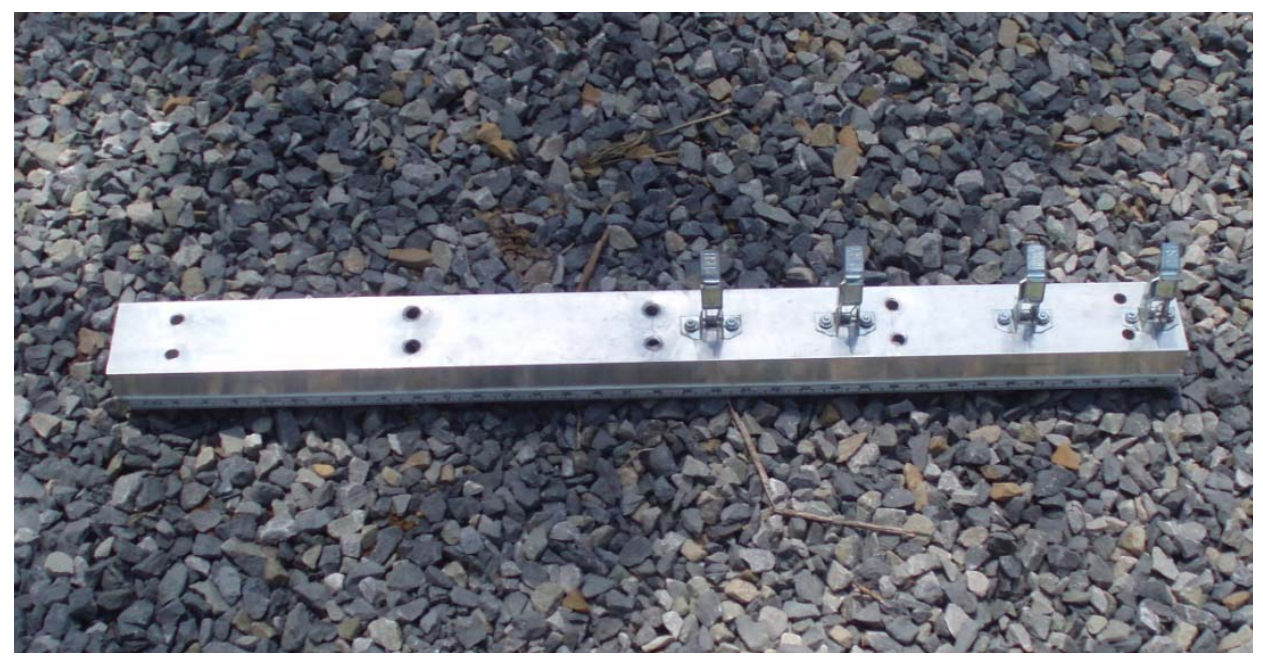

Figure 17: Traversing Mechanism Aluminum Beam

The traversing mechanism cart consists of a jack that allows the ADV to move in the vertical direction. Holes drilled though the aluminum beam allows it to connect to the bottom section the jack using two hex bolts and nuts (Figure 18). Spring loaded clamps on the aluminum beam allow the ADV to connect to the beam (Figure 19). The cart is placed on the bridge (Figure 20) and the traversing mechanism setup is complete. 


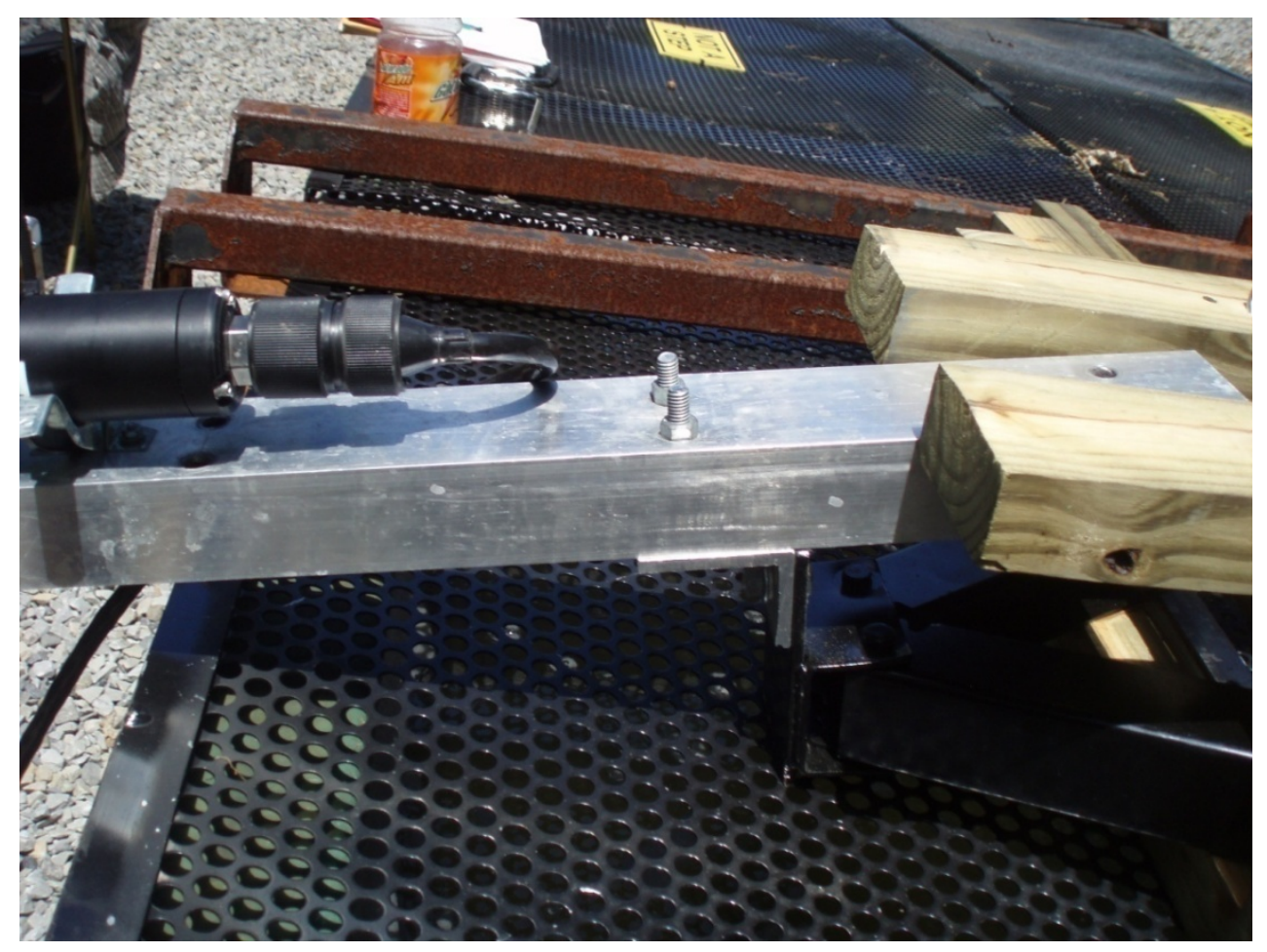

Figure 18: Connecting Aluminum Beam to Jack

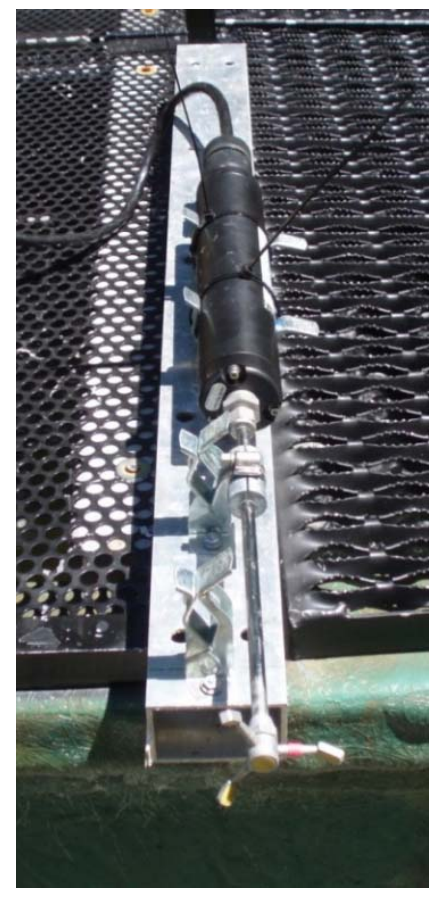

Figure 19: Connecting ADV to Aluminum Beam 


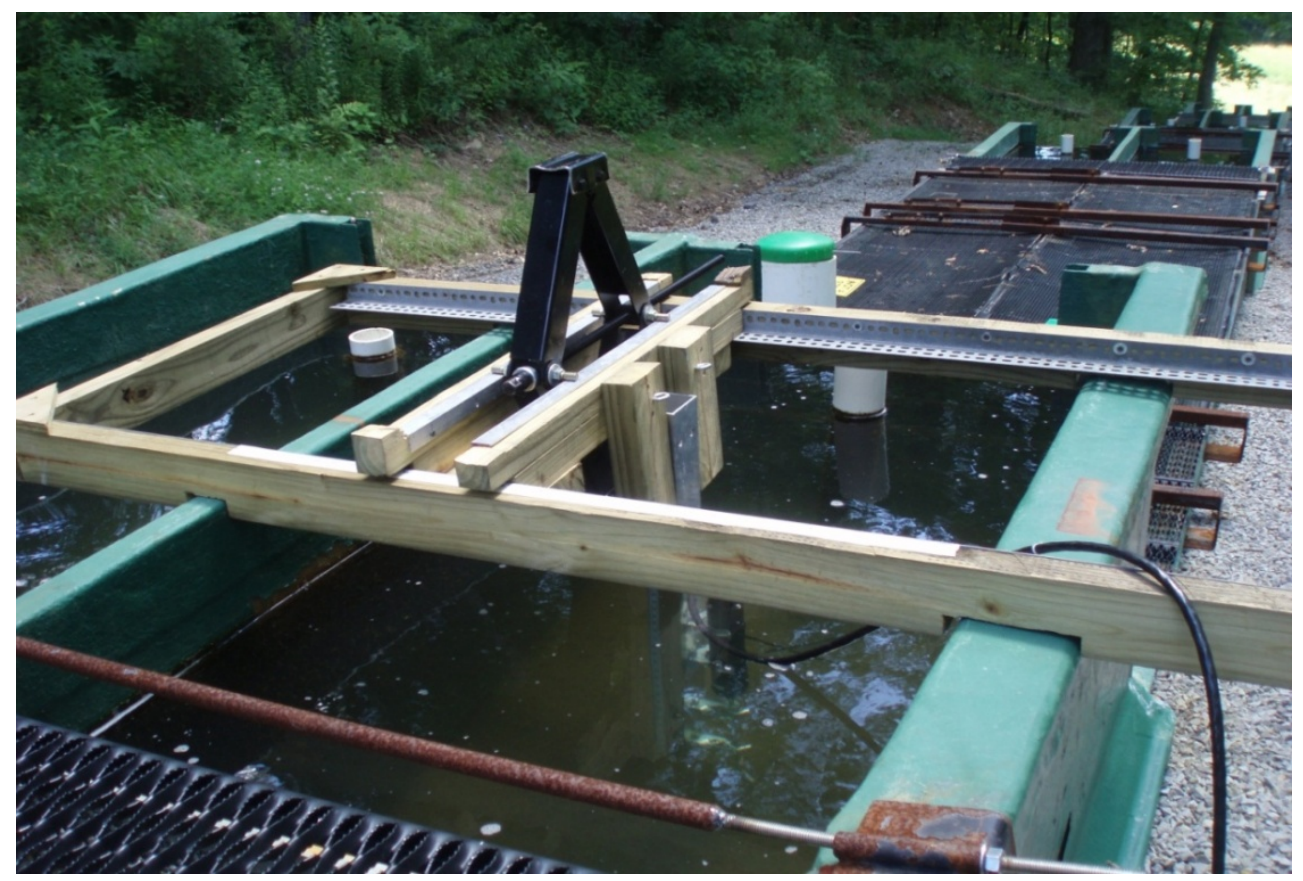

Figure 20: Complete Setup of Traversing Mechanism

The entire traversing mechanism system is designed specifically for the raceways at Dogwood Lake. The mechanism is capable of traversing within the quiescent zone in all three directions. Rotating the lead screw of the jack will move the probe in the vertical direction. The jack is very precise in its movement and can be moved in increments of $1 \mathrm{~mm}$. Measuring tape connected to the aluminum beam allows the movement of the ADV in the vertical direction to be measured. The ADV and provided software (SonTek, 2001) can detect the distance the probe and measuring volume is away from any boundary. This software provides a starting point for distance from the bottom surface. Sliding the cart along the tracks on the bridge allows for movement of the ADV in the transverse direction. Measuring tape is also connected to the bridge to allow for the movement to be measured in the transverse direction. The cart can accurately be moved along the bridge at increments of approximately $5 \mathrm{~mm}$. Finally, by moving the entire bridge along the frame of the quiescent zone, the ADV can traverse up and downstream. Streamwise movement is measured by connecting measuring tape to the frame of the quiescent zone. The bridge can accurately be moved along the quiescent zone in increments of approximately $5 \mathrm{~mm}$. 


\subsubsection{Traversing Mechanism Alignment Uncertainty}

When measuring different sets of data on different days it is important to be consistent in the location of the cart, bridge, and ADV. For each measurement set, waterproof tape is used to mark the starting location of the bridge on the quiescent zone, the cart on bridge, and the aluminum beam with respect to the cart. The ADV is carefully aligned on the aluminum beam to ensure consistent placement for each measurement set. A mark placed on the ADV is aligned with the spring loaded clamps (on the aluminum beam) in order to limit misalignment. Even with the marks, uncertainty of alignment must be estimated. An uncertainty of alignment is estimated to be $\pm 2 \mathrm{~cm}$ in all directions (streamwise, transverse, and vertical) with a $95 \%$ confidence interval. Rotational uncertainty of the ADV is estimated to be less than $\pm 1^{\circ}$ around the vertical axis with a 95\% confidence interval (discussed in Section 6.1.1). The affect of the misalignment of the traversing mechanism is calculated by averaging the change in mean velocities between measurements for each measured mean velocity profile (Section 8.0), and multiplying it by a factor of $0.2\left(\overline{\Delta \text { Mean Velocity }}\left(\frac{\mathrm{cm}}{\mathrm{s}}\right) \times 0.2\right)$. In all mean velocity profiles, the mean velocity measurements are separated by approximately $2.5 \mathrm{~cm}(2 \mathrm{~cm}$ is $80 \%$ of 2.5 $\mathrm{cm})$. The errors resulting from uncertainty of alignment in all directions for all profiles measured can be seen in Tables 4-6. The results show that misalignment will cause a small error for the profiles measured on different days. The mean transverse velocities, mean vertical velocities, and Reynolds stress will be the only measurements affected by this due to the relatively small magnitudes of these values.

Table 4: Streamwise Traversing Mechanism Uncertainty

\begin{tabular}{|c|c|c|c|c|c|c|c|c|}
\hline $\begin{array}{c}\bar{u} \\
(\mathrm{~cm} / \mathrm{s})\end{array}$ & $\begin{array}{c}\bar{v} \\
(\mathrm{~cm} / \mathrm{s})\end{array}$ & $\begin{array}{c}\bar{w} \\
(\mathrm{~cm} / \mathrm{s})\end{array}$ & $\begin{array}{c}\overline{u^{\prime 2}} \\
\left(\mathrm{~cm}^{2} / \mathrm{s}^{2}\right)\end{array}$ & $\begin{array}{c}\overline{v^{\prime 2}} \\
\left(\mathrm{~cm}^{2} / \mathrm{s}^{2}\right)\end{array}$ & $\begin{array}{c}\overline{w^{\prime 2}} \\
\left(\mathrm{~cm}^{2} / \mathrm{s}^{2}\right)\end{array}$ & $\begin{array}{c}\overline{u^{\prime} w^{\prime}} \\
\left(\mathrm{cm}^{2} / \mathrm{s}^{2}\right)\end{array}$ & $\begin{array}{c}\overline{u^{\prime} v^{\prime}} \\
\left(\mathrm{cm}^{2} / \mathrm{s}^{2}\right)\end{array}$ & $\begin{array}{c}\overline{v^{\prime} w^{\prime}} \\
\left(\mathrm{cm}^{2} / \mathrm{s}^{2}\right)\end{array}$ \\
\hline \pm 0.070 & \pm 0.060 & \pm 0.040 & \pm 0.0049 & \pm 0.0031 & \pm 0.0017 & \pm 0.0029 & \pm 0.0039 & \pm 0.0023 \\
\hline
\end{tabular}

Table 5: Transverse Traversing Mechanism Uncertainty

\begin{tabular}{|c|c|c|c|c|c|c|c|c|}
\hline $\begin{array}{c}\bar{u} \\
(\mathrm{~cm} / \mathrm{s})\end{array}$ & $\begin{array}{c}\bar{v} \\
(\mathrm{~cm} / \mathrm{s})\end{array}$ & $\begin{array}{c}\bar{w} \\
(\mathrm{~cm} / \mathrm{s})\end{array}$ & $\begin{array}{c}\overline{u^{\prime 2}} \\
\left(\mathrm{~cm}^{2} / \mathrm{s}^{2}\right)\end{array}$ & $\begin{array}{c}\overline{v^{\prime 2}} \\
\left(\mathrm{~cm}^{2} / \mathrm{s}^{2}\right)\end{array}$ & $\begin{array}{c}\overline{w^{\prime 2}} \\
\left(\mathrm{~cm}^{2} / \mathrm{s}^{2}\right)\end{array}$ & $\begin{array}{c}\overline{u^{\prime} w^{\prime}} \\
\left(\mathrm{cm}^{2} / \mathrm{s}^{2}\right)\end{array}$ & $\begin{array}{c}\overline{u^{\prime} v^{\prime}} \\
\left(\mathrm{cm}^{2} / \mathrm{s}^{2}\right)\end{array}$ & $\begin{array}{c}\overline{v^{\prime} w^{\prime}} \\
\left(\mathrm{cm}^{2} / \mathrm{s}^{2}\right)\end{array}$ \\
\hline \pm 0.10 & \pm 0.050 & \pm 0.060 & \pm 0.0096 & \pm 0.0027 & \pm 0.0035 & \pm 0.0058 & \pm 0.0051 & \pm 0.0031 \\
\hline
\end{tabular}


Table 6: Vertical Traversing Mechanism Uncertainty

\begin{tabular}{|c|c|c|c|c|c|c|c|c|}
\hline $\begin{array}{c}\bar{u} \\
(\mathrm{~cm} / \mathrm{s})\end{array}$ & $\begin{array}{c}\bar{v} \\
(\mathrm{~cm} / \mathrm{s})\end{array}$ & $\begin{array}{c}\bar{w} \\
(\mathrm{~cm} / \mathrm{s})\end{array}$ & $\begin{array}{c}\overline{u^{\prime 2}} \\
\left(\mathrm{~cm}^{2} / \mathrm{s}^{2}\right)\end{array}$ & $\begin{array}{c}\overline{v^{\prime 2}} \\
\left(\mathrm{~cm}^{2} / \mathrm{s}^{2}\right)\end{array}$ & $\begin{array}{c}\overline{w^{\prime 2}} \\
\left(\mathrm{~cm}^{2} / \mathrm{s}^{2}\right)\end{array}$ & $\begin{array}{c}\overline{u^{\prime} w^{\prime}} \\
\left(\mathrm{cm}^{2} / \mathrm{s}^{2}\right)\end{array}$ & $\begin{array}{c}\overline{u^{\prime} v^{\prime}} \\
\left(\mathrm{cm}^{2} / \mathrm{s}^{2}\right)\end{array}$ & $\begin{array}{c}\overline{v^{\prime} w^{\prime}} \\
\left(\mathrm{cm}^{2} / \mathrm{s}^{2}\right)\end{array}$ \\
\hline \pm 0.070 & \pm 0.070 & \pm 0.050 & \pm 0.0053 & \pm 0.0051 & \pm 0.0023 & \pm 0.0035 & \pm 0.0052 & \pm 0.0034 \\
\hline
\end{tabular}

\subsection{Preliminary Tests}

\subsubsection{Running Average Test}

Both Huggins (2003) and Rumberg (2004) took mean velocity measurements in quiescent zones. Huggins averaged velocity measurements for 30-40 seconds at a sampling rate of $1 \mathrm{~Hz}$ in order to measure mean velocities. Rumberg averaged velocities for 10 seconds at a sampling rate of $1 \mathrm{~Hz}$ in order to measure mean velocities. Rumberg took running averages of all three velocity components at Dogwood Lake, but they showed that a 10 second averaging time is inadequate for determining accurate mean velocities. Huggins did not provide a running average to prove that his sampling time was adequate to accurately measure mean velocities. The purpose of the running average test in this section is to prove that the averaging time chosen for this research is long enough to accurately measure mean velocities.

A running average is taken in the center of the quiescent zone in order to determine the length of the sampling time required to accurately measure mean streamwise velocities. In Figure 12, a running average of the streamwise velocities is measured approximately $43 \mathrm{~cm}$ from the bottom of a quiescent zone at Dogwood Lake. Figure 12 shows that a mean velocity can be established with approximately 200 seconds of sampling at $10 \mathrm{~Hz}$. SonTek's Micro ADV is capable of sampling up to $50 \mathrm{~Hz}$; however, a $10 \mathrm{~Hz}$ frequency is used to limit noise. In order to ensure confidence that a mean velocity is accurately measured, a sampling time of 360 seconds is used for all of the velocity measurements at Dogwood Lake.

\subsubsection{ADV Rotational Sensitivity Test}

Human error may cause slight magnitude changes with respect to the velocity measurements. This includes the difficulty of perfectly aligning the ADV with the mean flow. When using the traversing mechanism described above, slight misalignment may occur (less than $\pm 1^{\circ}$ ). It is important to determine the effects of a misaligned ADV with the mean flow. 
Misalignment is most likely to occur with respect to rotation around the $z$-axis. The following tests were conducted to determine the effect of misalignment of the ADV about the $z$-axis using the Micro ADV.

A test must be conducted to determine the angular sensitivity (rotation around $z$-axis) of the ADV. Using a laboratory flume and PVC flow straighter, the ADV measured mean velocities at various angles around the $z$-axis (coordinate system shown in Figure 1). Each mean velocity is averaged over a 120 second time interval and is sampled at ten samples per second. An angle of zero degrees represents the ADV probe directly in line with the mean flow. Figures 21-23 demonstrate the angular sensitivity of the ADV for each velocity component. A positive rotation is considered clockwise around the $z$-axis. Due to size limitations of the laboratory flume, a positive rotation is the only rotational sensitivity measured. Assuming the sensitivity ( $z$ axis rotation) of the mean velocities $(\bar{u}, \bar{v}, \bar{w})$ to be the same for both negative and positive rotation allows negative sensitivity to be shown in the plots. Sensitivity for negative $z$-axis rotation (counter-clockwise) is assumed to have the same magnitude as the positive sensitivity but acting in the opposite direction. 


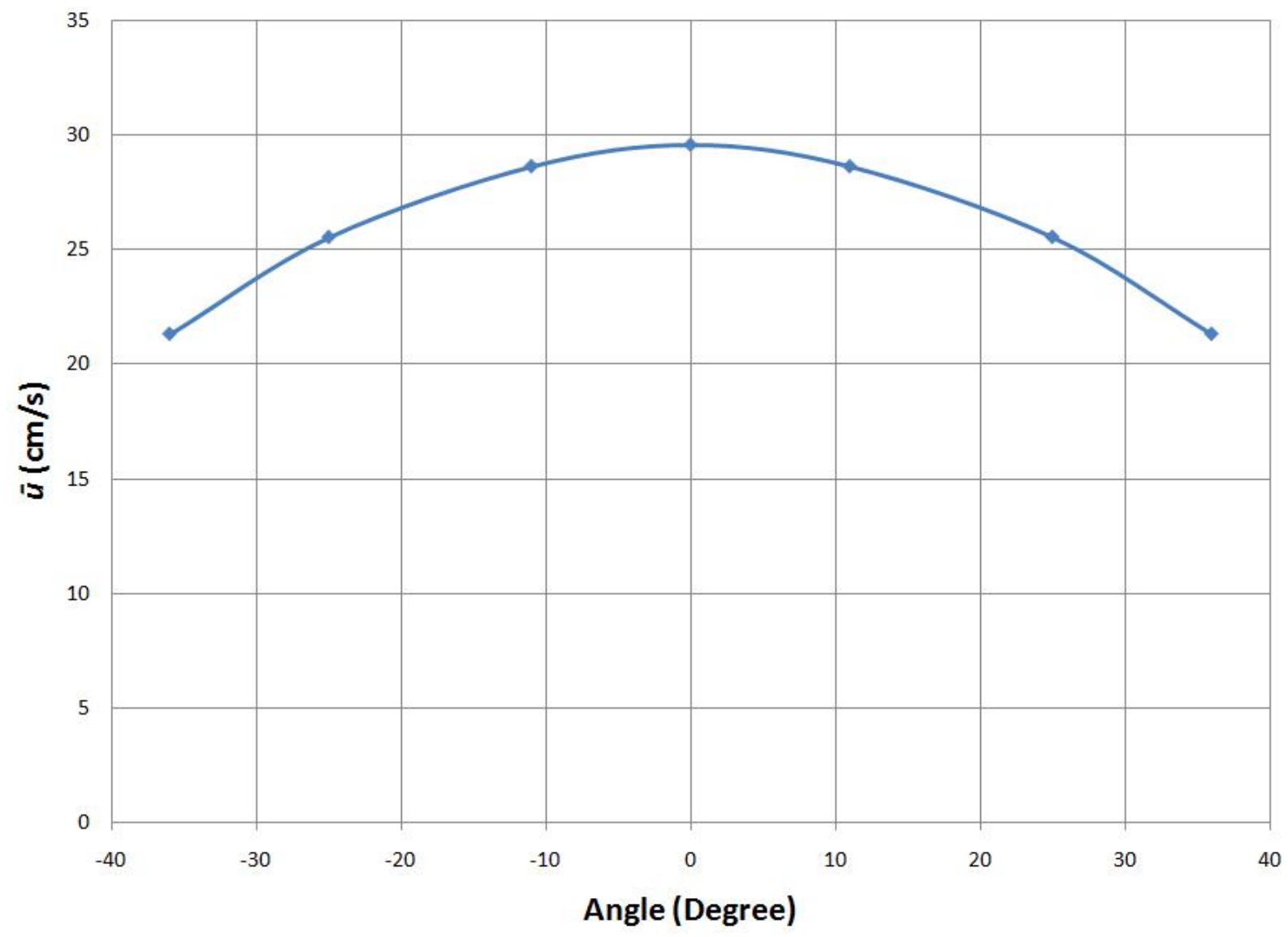

Figure 21: Mean Streamwise Velocity Angular Sensitivity 


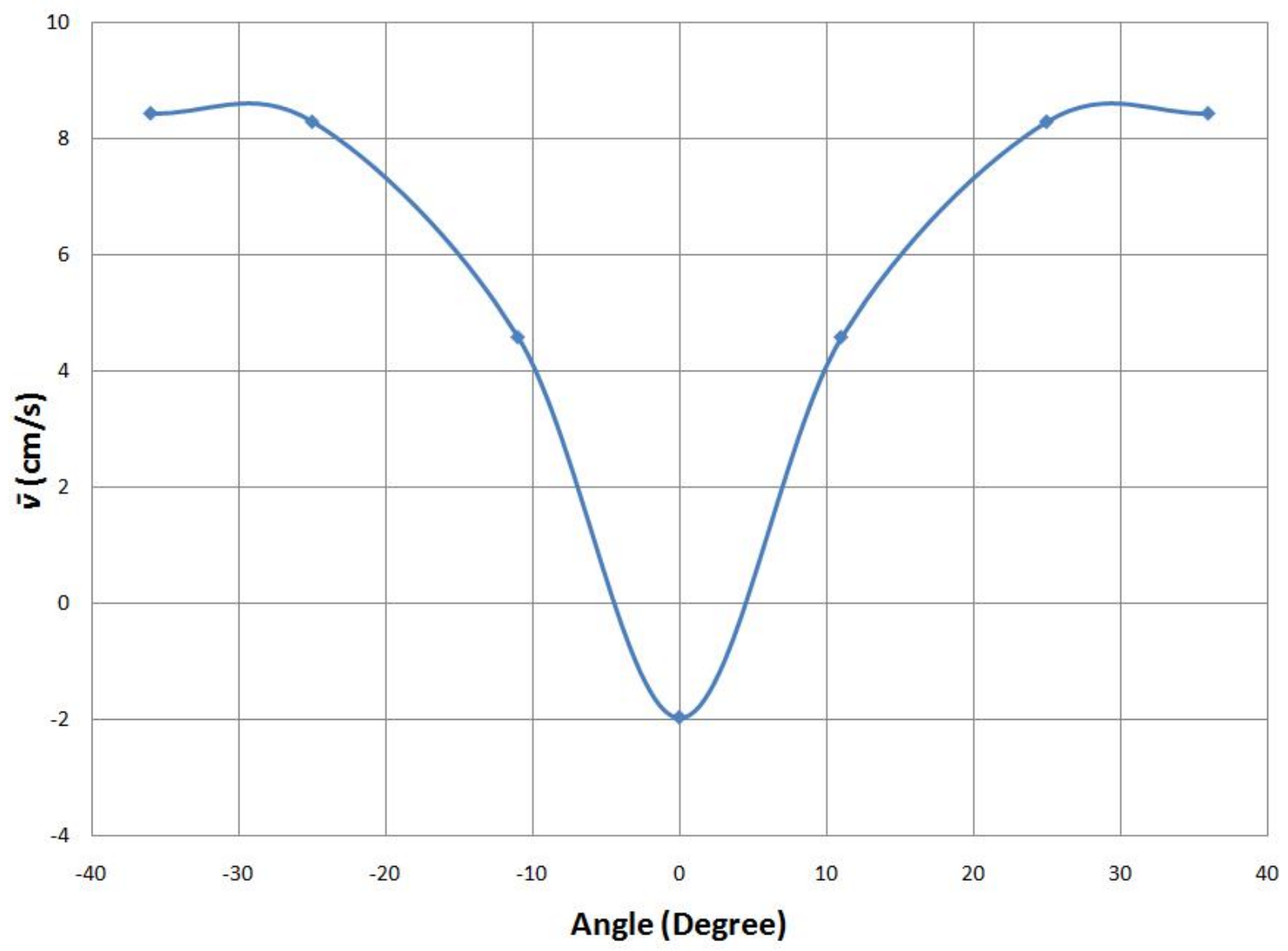

Figure 22: Mean Transverse Velocity Angular Sensitivity 


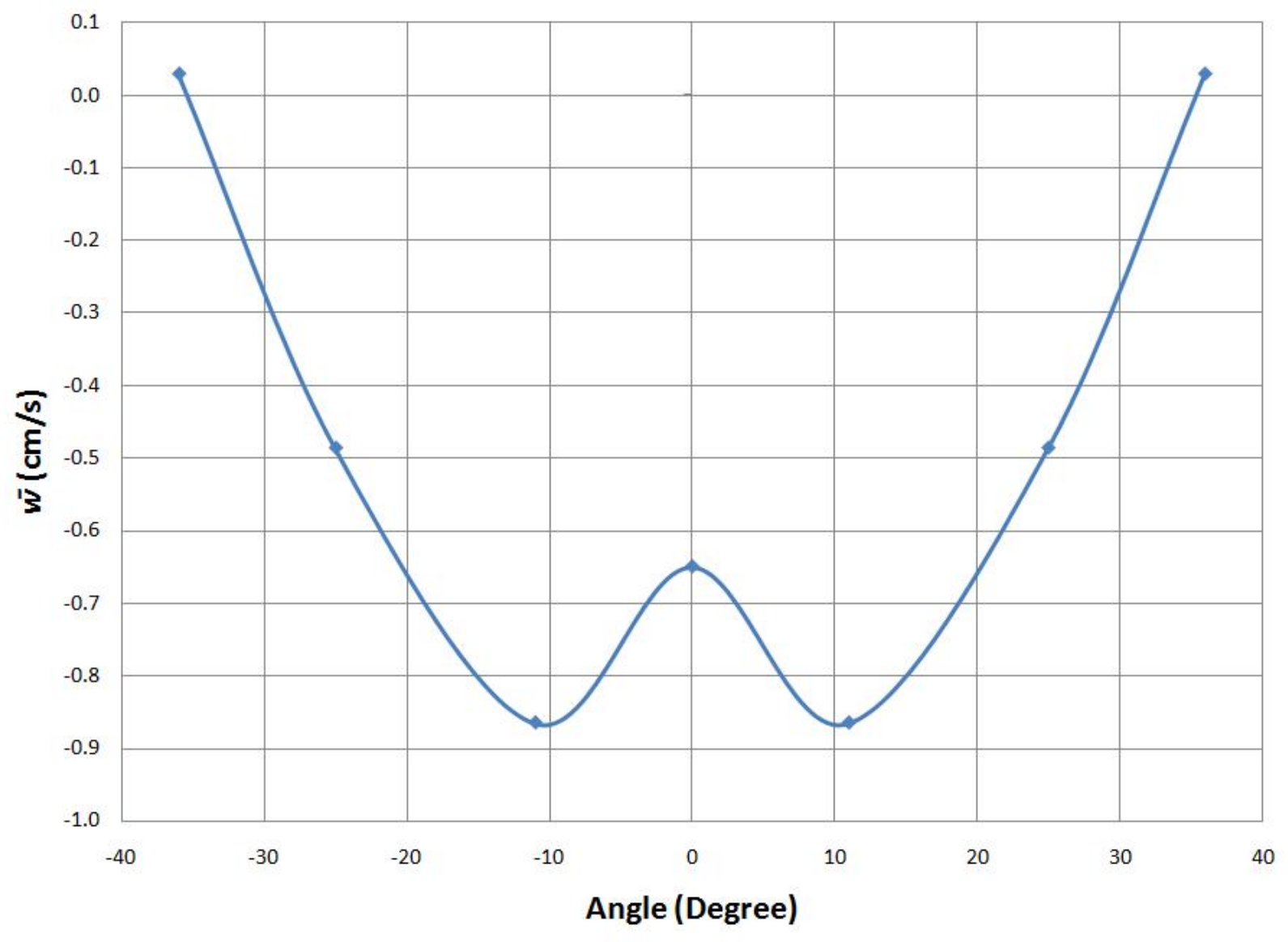

Figure 23: Mean Vertical Velocity Angular Sensitivity

Table 7: Angular Sensitivity with Respect to Small Rotations around the Z-Axis

\begin{tabular}{|c|c|c|}
\hline $\bar{u}$ Sensitivity (cm/s/degree) & $\overline{\bar{v}}$ Sensitivity (cm/s/degree) & $\overline{\bar{w}}$ Sensitivity (cm/s/degree) \\
\hline-0.080 & +0.59 & -0.21 \\
\hline
\end{tabular}

Table 7 shows the sensitivity of the ADV used for this research while approximating the sensitivity curves in Figures 21-23 to be linear for small rotations around the $z$-axis (small rotations are considered 0-11 degrees). Table 7 indicates that the mean streamwise velocity is not severely impacted by small rotations due to misalignment of the probe. Conversely, even with misalignments less than $\pm 1^{\circ}$, transverse and vertical velocities will be affected by angular sensitivity. 


\subsection{Raceway Limitations at Dogwood Lake}

As mentions in Section 4.2.1 and 4.2.2 the length and aspect ratio are factors that determine the ability of an open channel to produce two-dimensional, fully developed flow. The length of the raceway must be long enough to allow fully developed flow characteristics to occur. As stated in Section 4.2.1, a distance from the inlet to the measuring location should be 50-100 times the depth of the channel in order to receive fully developed characteristics (Trowbridge et al., 1989). The longest distance of approximately 9.0 meters downstream of the inlet, and water depth of $89 \mathrm{~cm}$, results in a value of approximately 10 times the depth of the water. As stated in Section 4.2.2, the aspect ratio of the open channel (width of channel to depth of channel) should be approximately 6 to achieve two-dimensional characteristics (Nakagawa et al, 1983). This aspect ratio at Dogwood Lake is approximately 1 . The geometry of the Dogwood Lake raceway system does not meet the requirements to achieve two-dimensional, fully developed, turbulent open channel flow over smooth surfaces.

The theoretical/empirical calculations consider flow over smooth surfaces. The surfaces

of the raceway system at Dogwood Lake are made of fiberglass containing small dimples, a large amount of algae growth on the walls, and settled solids on the bottom surface. These factors will contribute to discrepancies between the theoretical/empirical characteristics and measured characteristics.

\subsection{Measurements and Discussion}

\subsubsection{Description of Quiescent Zones and Collected Measurements}

Data is collected in different quiescent zones (details shown in Table 8) in order to compare the affects that fish and different separating screen types have on the flow characteristics.

There are two types of screens that separate the quiescent zone from the fish area; a small mesh screen and a large mesh screen. The small and large screens are identical other than a plastic square mesh that is placed behind the large screen to decrease the openings. Pictures and dimensions for the screens can be seen in Figure 24-25. It should be noted that debris easily accumulates and causes blockage in the screens. Prior to each measurement set (with the exception of measurements taken on September 30, 2008), the screens are cleaned using a 
broom. The debris is difficult to remove entirely due to its cohesive properties. It should also be noted that the screens may not have been cleaned to the same degree before each measurement set and this may cause discrepancies between measurements taken on different days. Also, it should be noted that a larger screen allows for more thorough removal of debris.

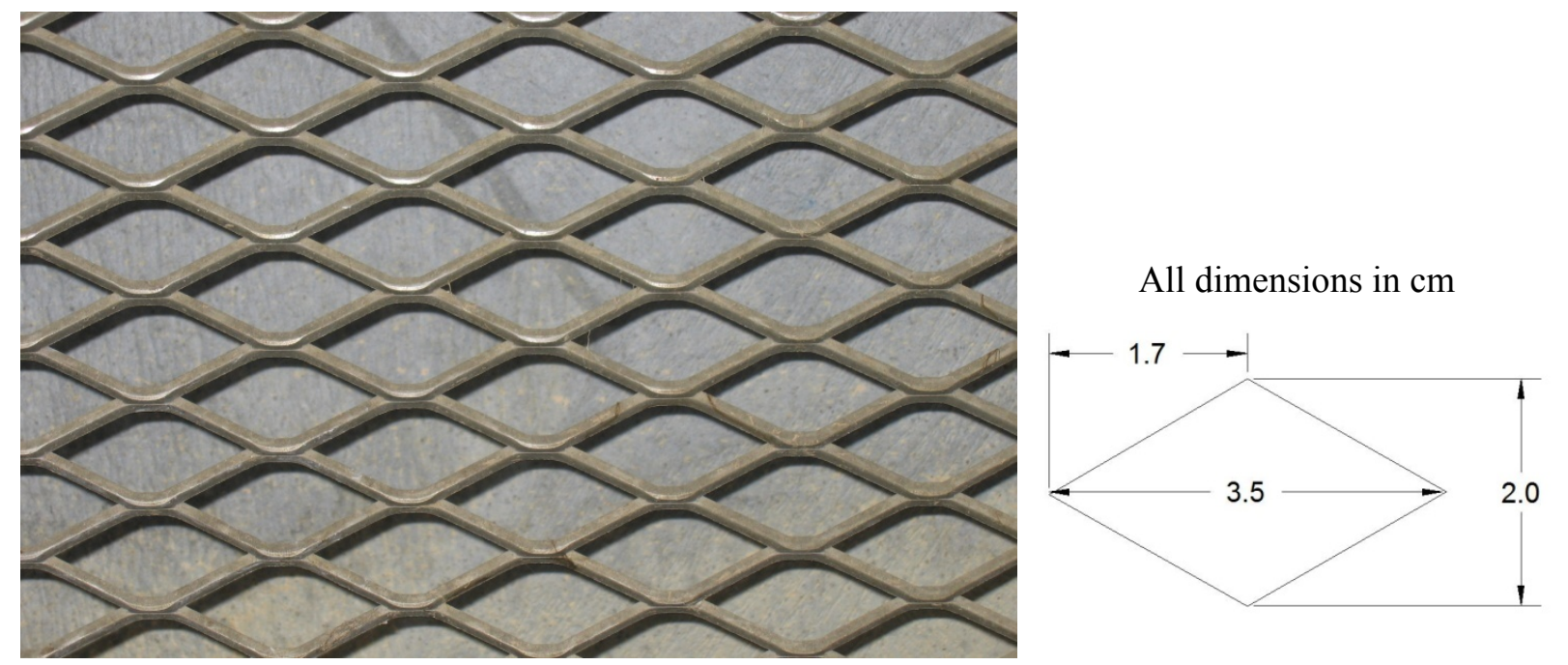

Figure 24: Large Screen Used to Separate Fish Zone from Quiescent Zone 1 and Corresponding Mesh Dimensions
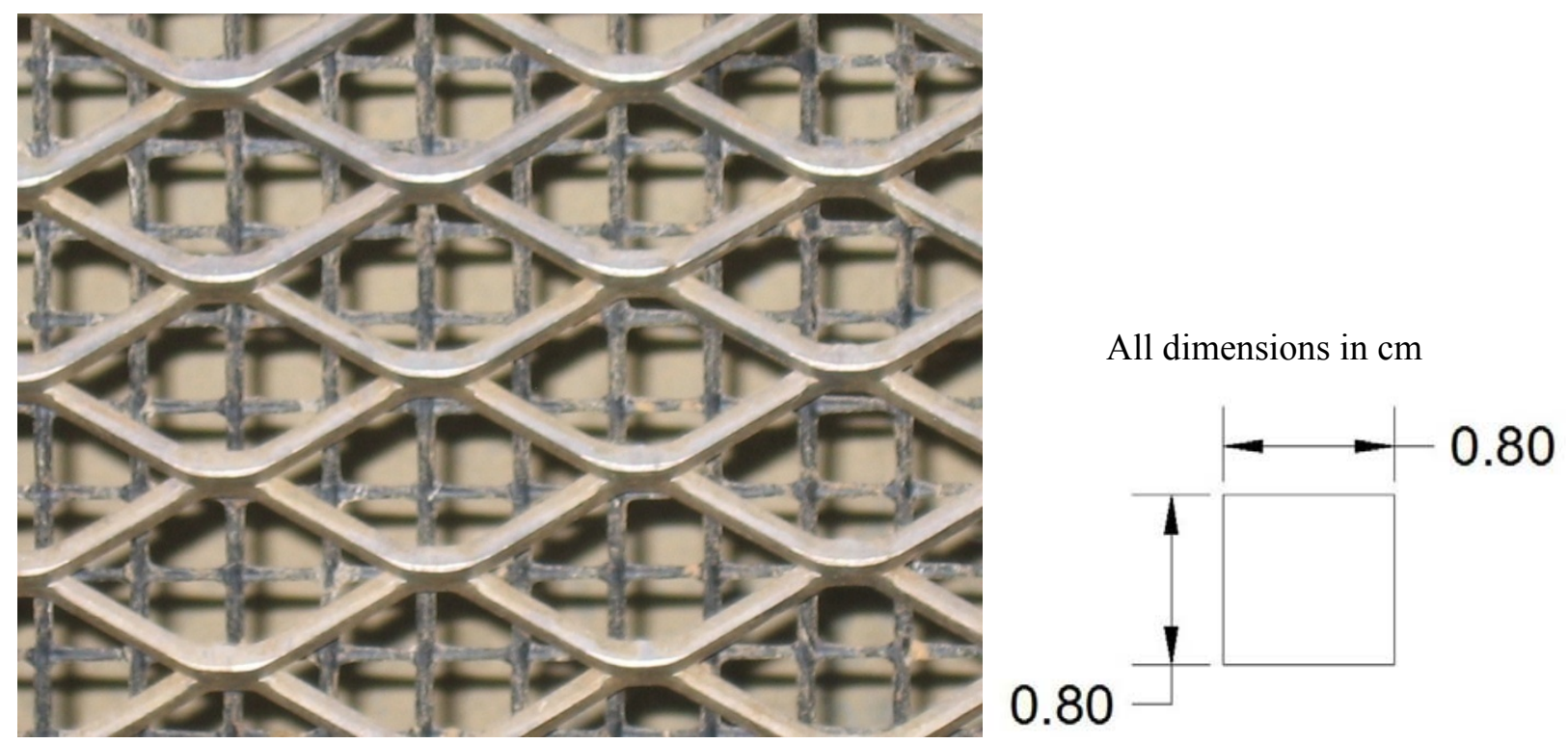

Figure 25: Small Screen Used to Separate the Fish Zone from Quiescent Zone 2 and Corresponding Mesh Dimensions 


\subsubsection{Collected Measurements}

All of the measurements were taken using SonTek's Micro ADV. At each measurement location the ADV sampled velocities at $10 \mathrm{~Hz}$ with a velocity range of $\pm 10 \mathrm{~cm} / \mathrm{s}$. The previous velocity range is chosen since free surface velocities were calculated using a float at values slightly larger than $3.0 \mathrm{~cm} / \mathrm{s}$. Each mean velocity is averaged over a 360 second time interval.

A description of the collected measurements can be seen in Table 8 and Figures 26-33. The flow rates are measured prior to each measurement set with the exception of measurements taken on September 30, 2008. The flow rates were measured using a $12 \times 10^{4} \mathrm{~cm}^{3}$ tub, and timing how long it took to fill. 2 to 3 trials were conducted and averaged to receive the flow rates in Table 8 . The flow rates vary due to the daily changes in flow. The wind speeds in Table 8 were measured using a Vanometer manufactured by Dwyer Instruments. A Vanometer allows for an approximation of the wind speed.

Table 8: Description of Collected Measurements

\begin{tabular}{|c|c|c|c|c|c|c|c|c|}
\hline Date & Figure & QZ & Fish & Screen Type & Profile & \# of Measurements & Flow Rate (cm3/s) & Wind (m/s) \\
\hline 30-Aug & 15 & A4 & 0 & Large & Vertical & 25 & $16 \times 10^{3}$ & $<0.7$ \\
\hline 4-Sep & 16 & A4 & 0 & Large & Vertical & 30 & $16 \times 10^{3}$ & $<0.1$ \\
\hline 30-Sep & 17 & A2 & 5000 Striped Bass & Small Dirty & Vertical & 20 & No Data & $<0.1$ \\
\hline 9-Oct & 18 & A2 & 5000 Striped Bass & Small & Vertical & 23 & $17 \times 10^{3}$ & $<0.3$ \\
\hline 4-Nov & 19 & A2 & 5000 Striped Bass & Small & Transverse & 31 & $13 \times 10^{3}$ & $<0.1$ \\
\hline 6-Nov & 20 & A4 & 0 & Large & Transverse & 30 & $13 \times 10^{3}$ & $<0.4$ \\
\hline 26-Nov & 21 & A2 & 5000 Striped Bass & Small & Streamwise & 23 & $10 \times 10^{3}$ & $<0.5$ \\
\hline 29-Nov & 22 & B4 & 0 & Large & Streamwise & 25 & $15 \times 10^{3}$ & $<0.6$ \\
\hline
\end{tabular}



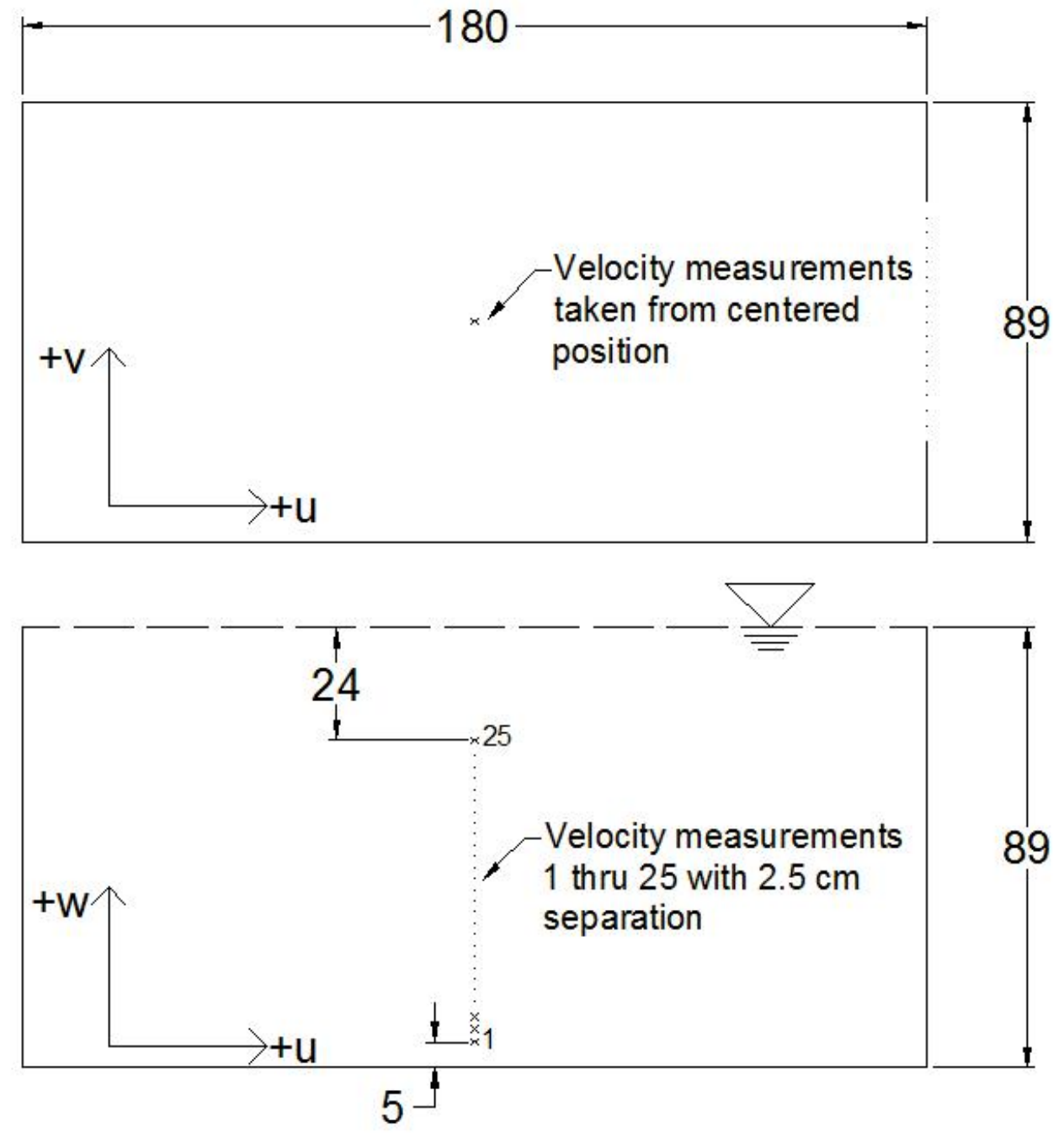

${ }^{\star}$ Note: All dimensions in $\mathrm{cm}$

Figure 26: Location of Vertical Profile Measurements taken on August 30, 2008 


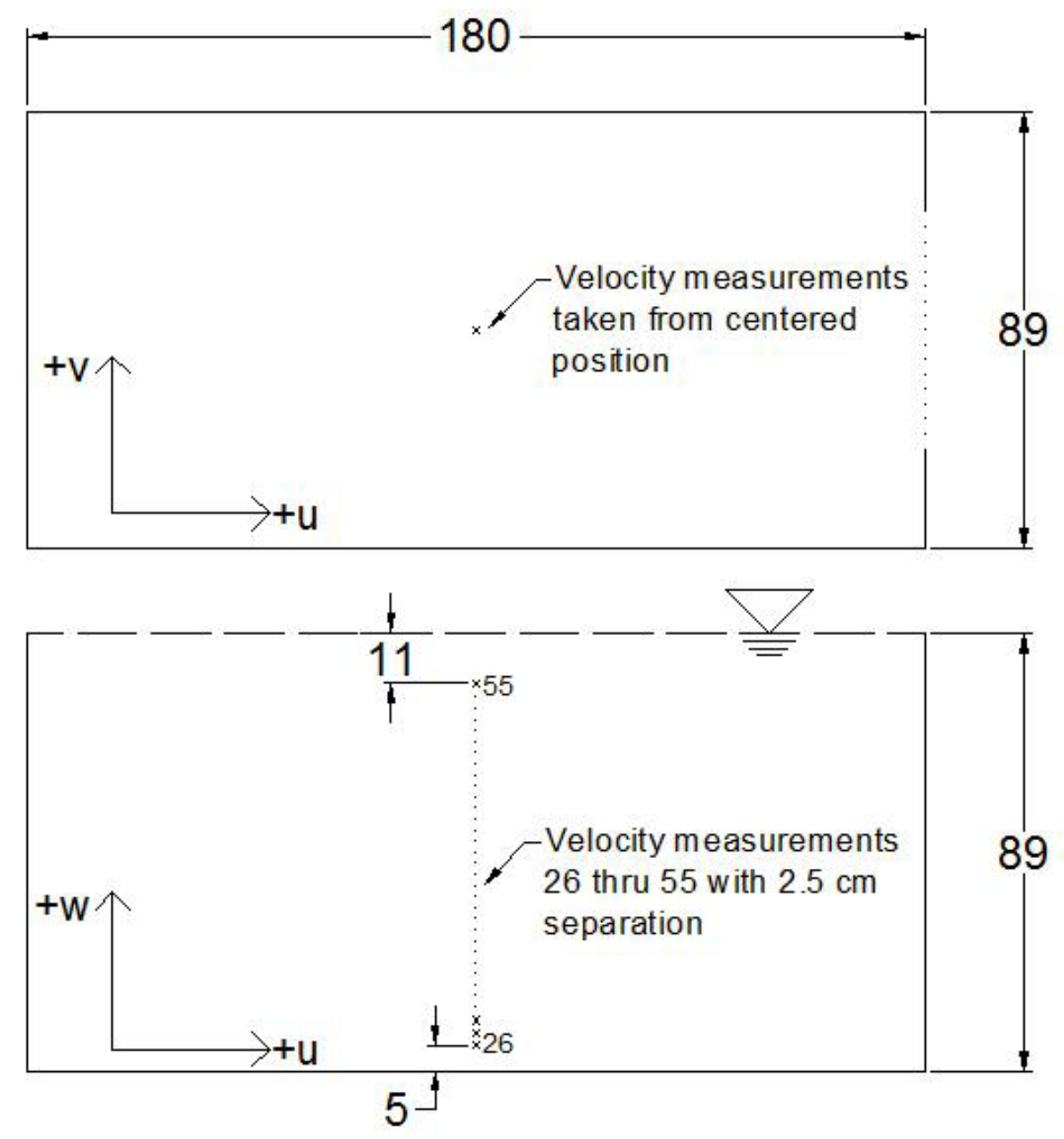

${ }^{*}$ Note: All dimensions in $\mathrm{cm}$

Figure 27: Location of Vertical Profile Measurements taken on September 4, 2008 

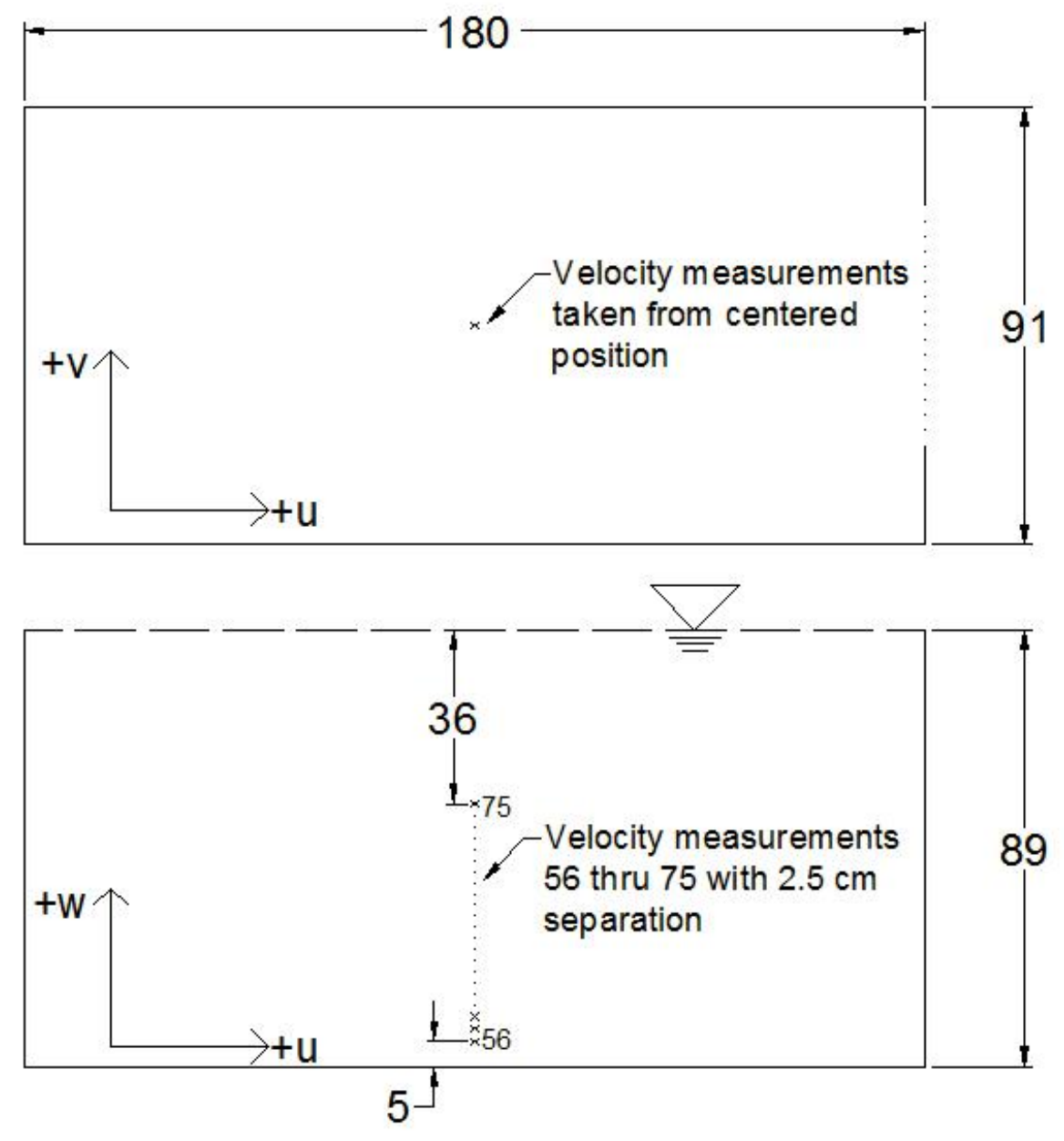

${ }^{\star}$ Note: All dimensions in $\mathrm{cm}$

Figure 28: Location of Vertical Profile Measurements taken on September 30, 2008 


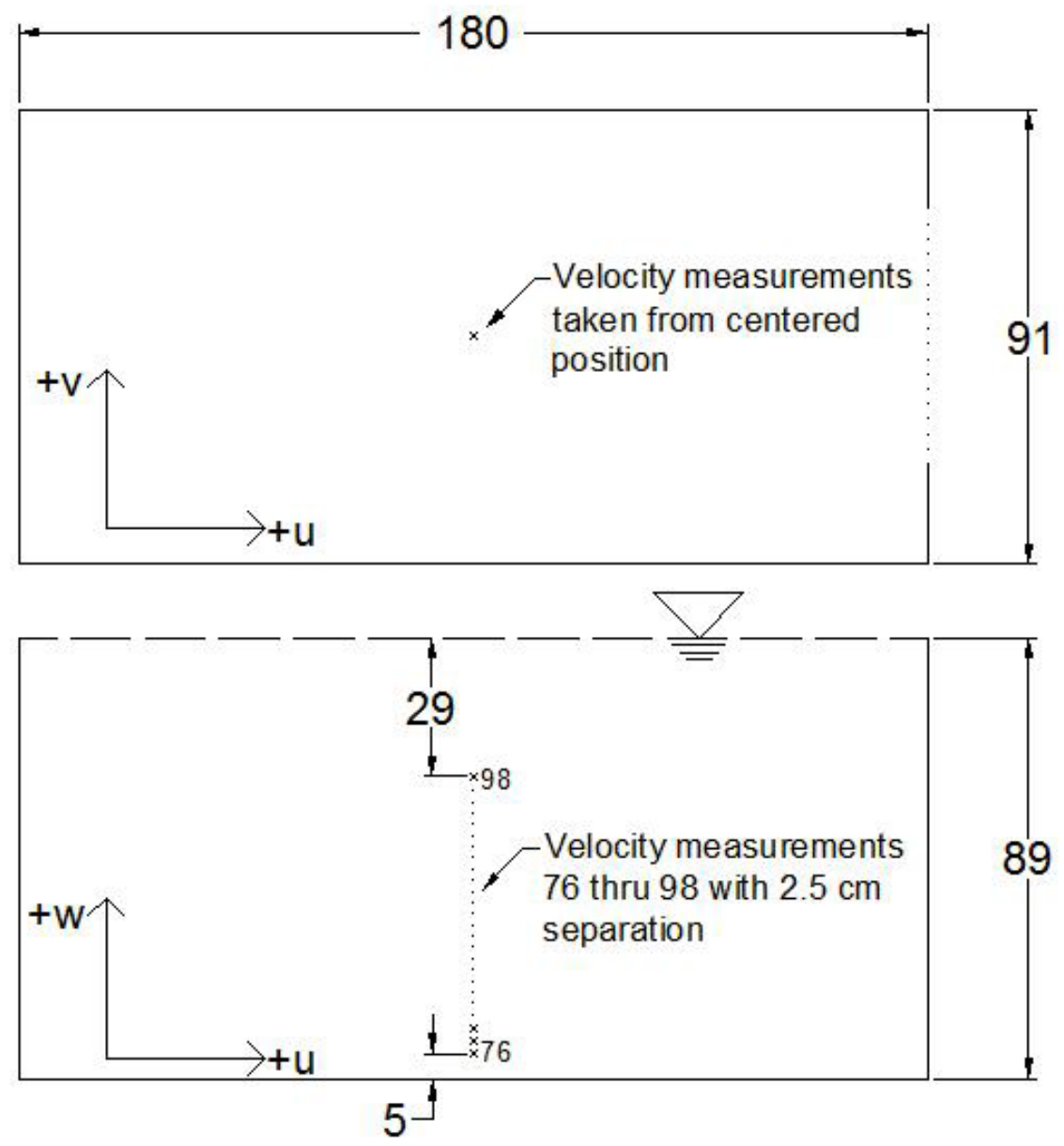

*Note: All dimensions in $\mathrm{cm}$

Figure 29: Location of Vertical Profile Measurements taken on October 9, 2008 


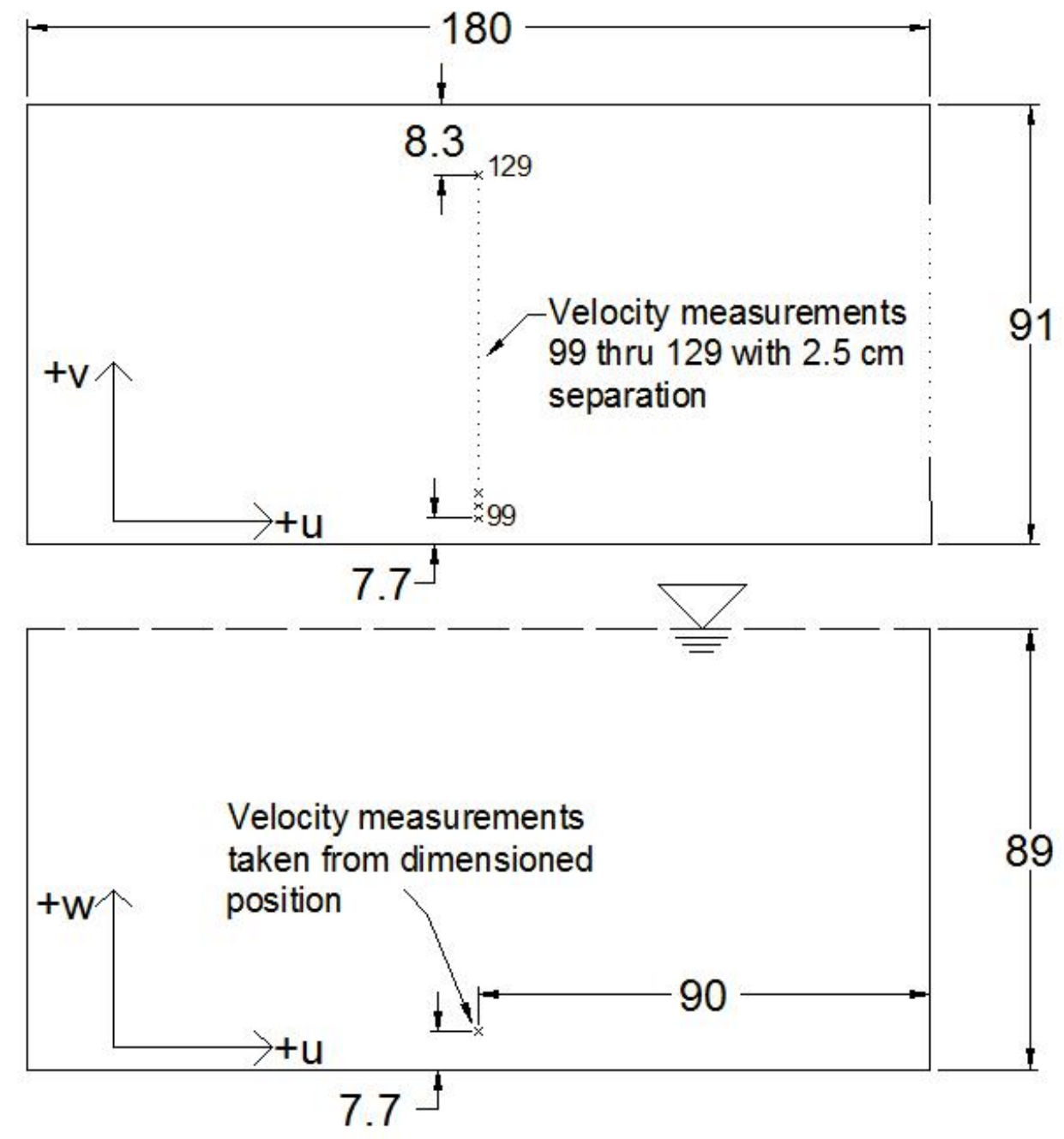

${ }^{\star}$ Note: All dimensions in $\mathrm{cm}$

Figure 30: Location of Transverse Profile Measurements taken on November 4, 2008 

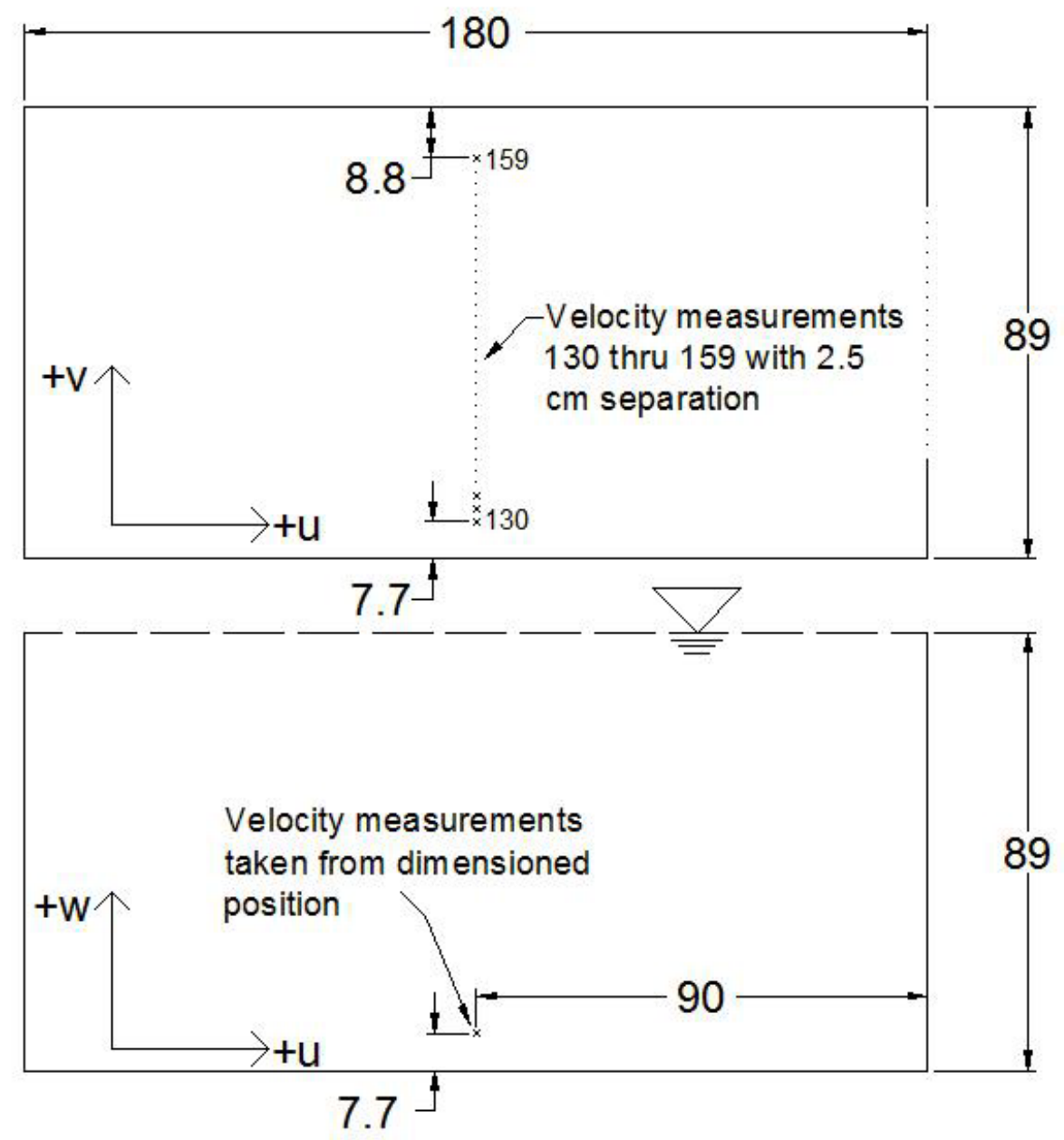

${ }^{\star}$ Note: All dimensions in $\mathrm{cm}$

Figure 31: Location of Transverse Profile Measurements taken on November 6, 2008 


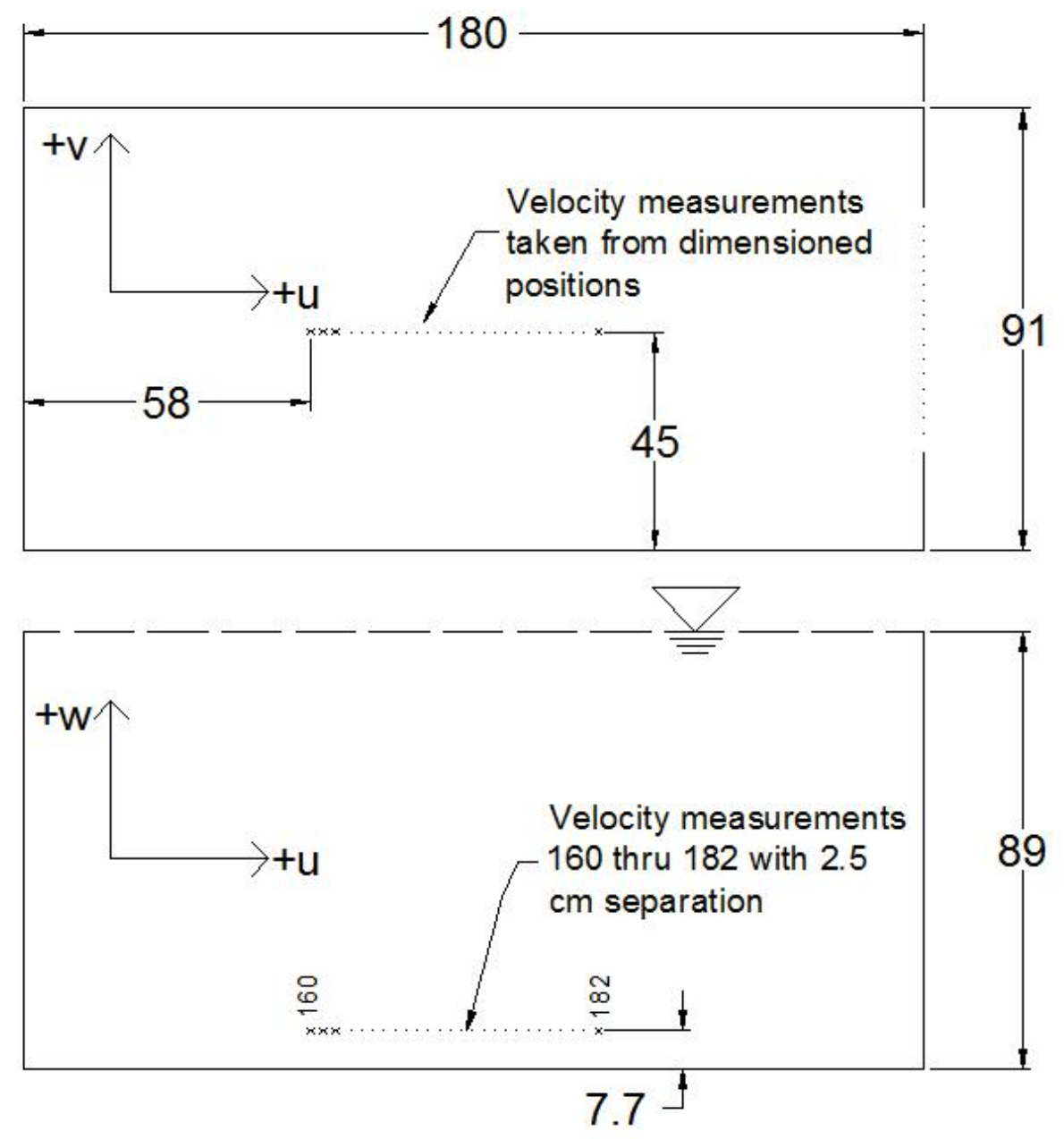

*Note: All dimensions in $\mathrm{cm}$

Figure 32: Location of Streamwise Profile Measurements taken on November 26, 2008 


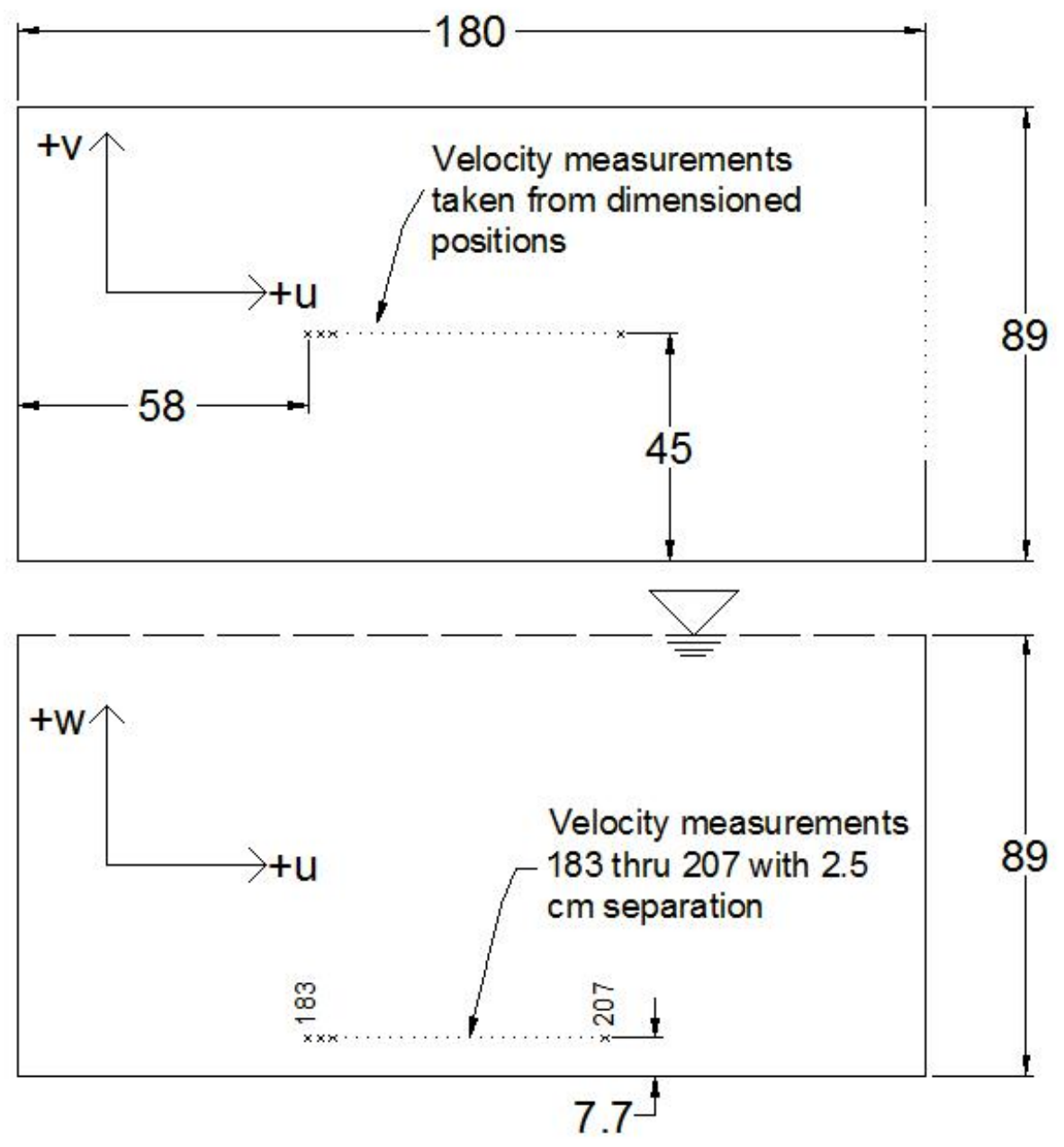

${ }^{\star}$ Note: All dimensions in $\mathrm{cm}$

Figure 33: Location of Streamwise Profile Measurements taken on November 29, 2008

\subsubsection{Vertical Profiles of Mean Velocity}

For each vertical mean velocity profile, the initial measurement point is located approximately $5 \mathrm{~cm}$ from the bottom of the quiescent zone, and each measurement thereafter is approximately $2.5 \mathrm{~cm}$ from the previous. In several attempts to measure closer to the bottom, $(<$ $5 \mathrm{~cm}$ ) the ADV outputted unrealistic results with low correlation values. For this reason, $5 \mathrm{~cm}$ is used as the limit for the minimum distance from the bottom. The profiles end at varying locations from the free surface due to occasional problems with the ADV that required a substantial amount of time to fix, resulting in the laptop losing its battery charge. 
The black curve in Figures 34, 38, and 39 represents the fully developed, turbulent open channel mean vertical velocity profile at the center of the channel, for flow over a smooth surface. The theoretical/empirical black curve is calculated by considering the viscous sub-layer and turbulent layer (Equations 31-33). The free surface is represented by a blue horizontal line in all vertical mean velocity profiles.

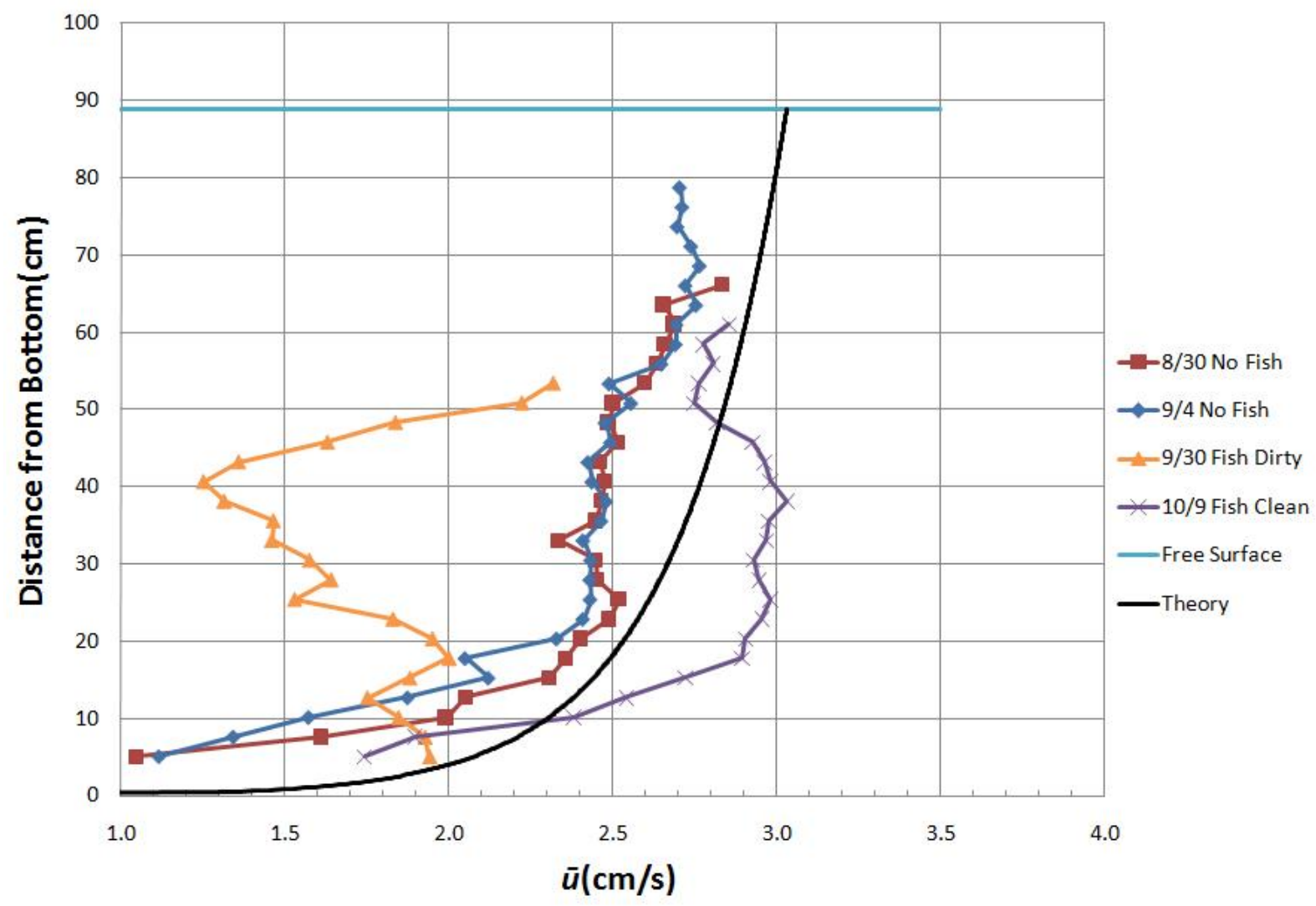

Figure 34: Vertical Profiles of Mean Streamwise Velocity

Figure 34 shows vertical profiles of the mean streamwise velocity corresponding to Figures 2629.

The mean velocity profiles measured on August 30, September 4, and October 9 share a similar shape with the theoretical/empirical vertical mean streamwise velocity profile; however, each measured profile varies from the theoretical/empirical profile with respect to smoothness and magnitude. These differences may be due to the limited length of the raceway system, and disturbances such as upstream presence of the screen, and in some cases, fish. The profile measured on September 30 bears no resemblance to the theoretical/empirical profile. Since the 
profile on September 30 is measured downstream of a screen that contains debris, it can be stated that this debris may be causing the mean streamwise velocity profile to drastically differ from measurements taken downstream of a clean screen.

The profile measured on October 9 varies from the profiles measured on August 30 and September 4, showing that the screen type and presence of fish upstream may be the cause of the visible difference in the measured mean streamwise velocity profiles.

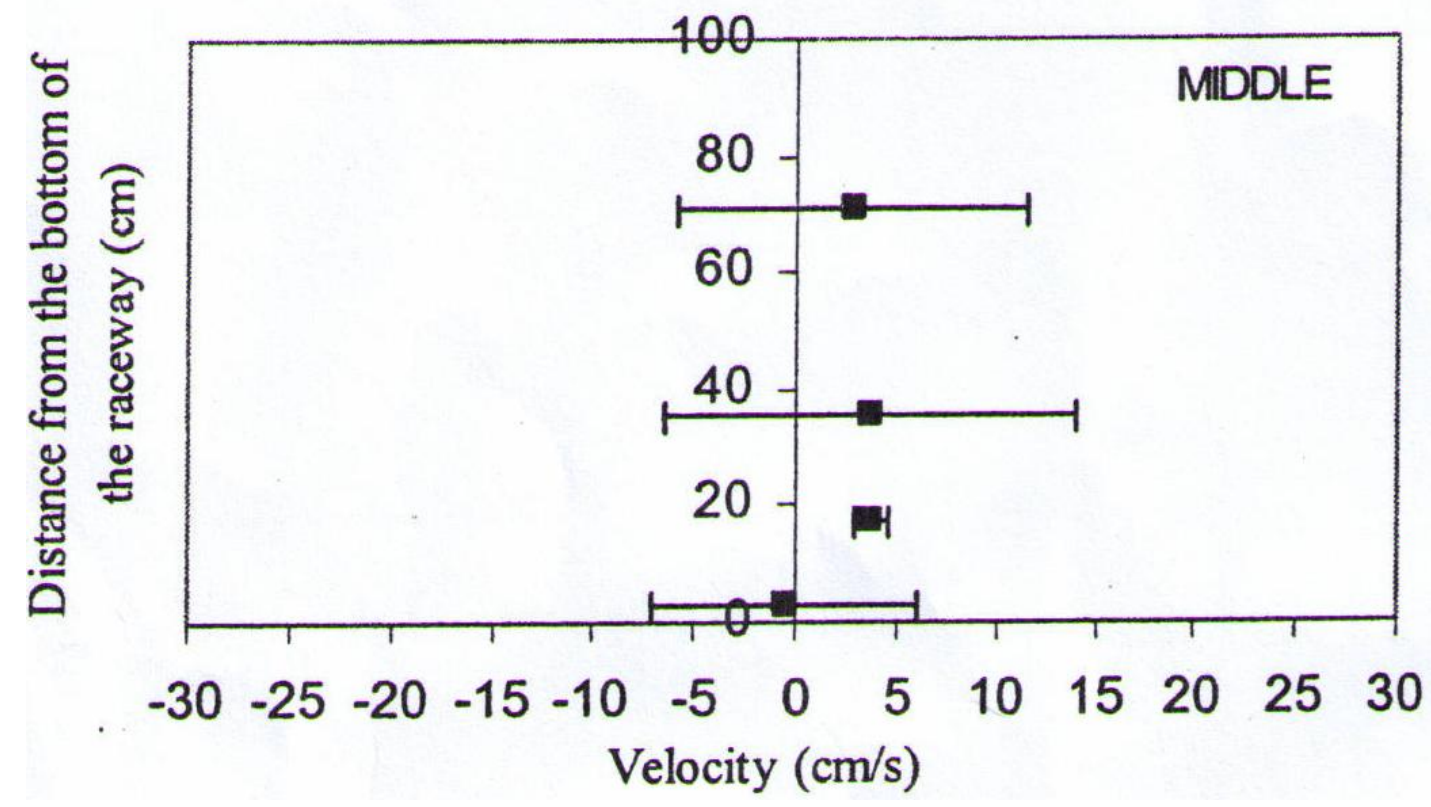

Figure 35: Huggins' Vertical Profile of Mean Streamwise Velocity (Huggins, 2003)

Figure 35 shows a vertical profile of the mean streamwise velocity measured by Huggins (2003) at a location near the center of the quiescent zone used in his research. The depth of the water in the quiescent zone used for this measurement set is approximately $0.91 \mathrm{~m}$. The bars running through each measurement point in Figure 35 represent the standard deviations of the measured mean streamwise velocities.

Huggins used SonTek's FlowTracker ADV to measure mean velocities. The FlowTracker pings a maximum of 10 times per second and averages them to produce a velocity measurement every second. The FlowTracker has a resolution of $0.1 \mathrm{~cm} / \mathrm{s}$, which is 10 times less precise than the Micro ADV (resolution of $0.01 \mathrm{~cm} / \mathrm{s}$ ). Depending on the level of turbulence, Huggins' averaging time ranged from 30-40 seconds. A 30-40 second averaging time range may be adequate for measuring mean velocities in the raceway system used in Huggins' research, though this range is short with respect to the averaging time required by the Micro ADV at Dogwood 
Lake. The magnitudes of velocity seem to be similar to the magnitudes measured in Figure 34, and the width of the raceway system Huggins used is slightly larger than at Dogwood Lake. A Reynolds number of approximately $4 \times 10^{3}$ is estimated for the raceway system used by Huggins. Since the Reynolds number at Dogwood Lake is approximately half the magnitude of the Reynolds number approximated at Huggins raceway system; an averaging time of 30-40 seconds is too short to capture accurate mean streamwise velocities. Huggins could also use a more detailed mean velocity profile to determine any changes in flow between measurements. Also, a larger sampling and ping rate may be required by Huggins to capture small scale turbulence and limit noise.

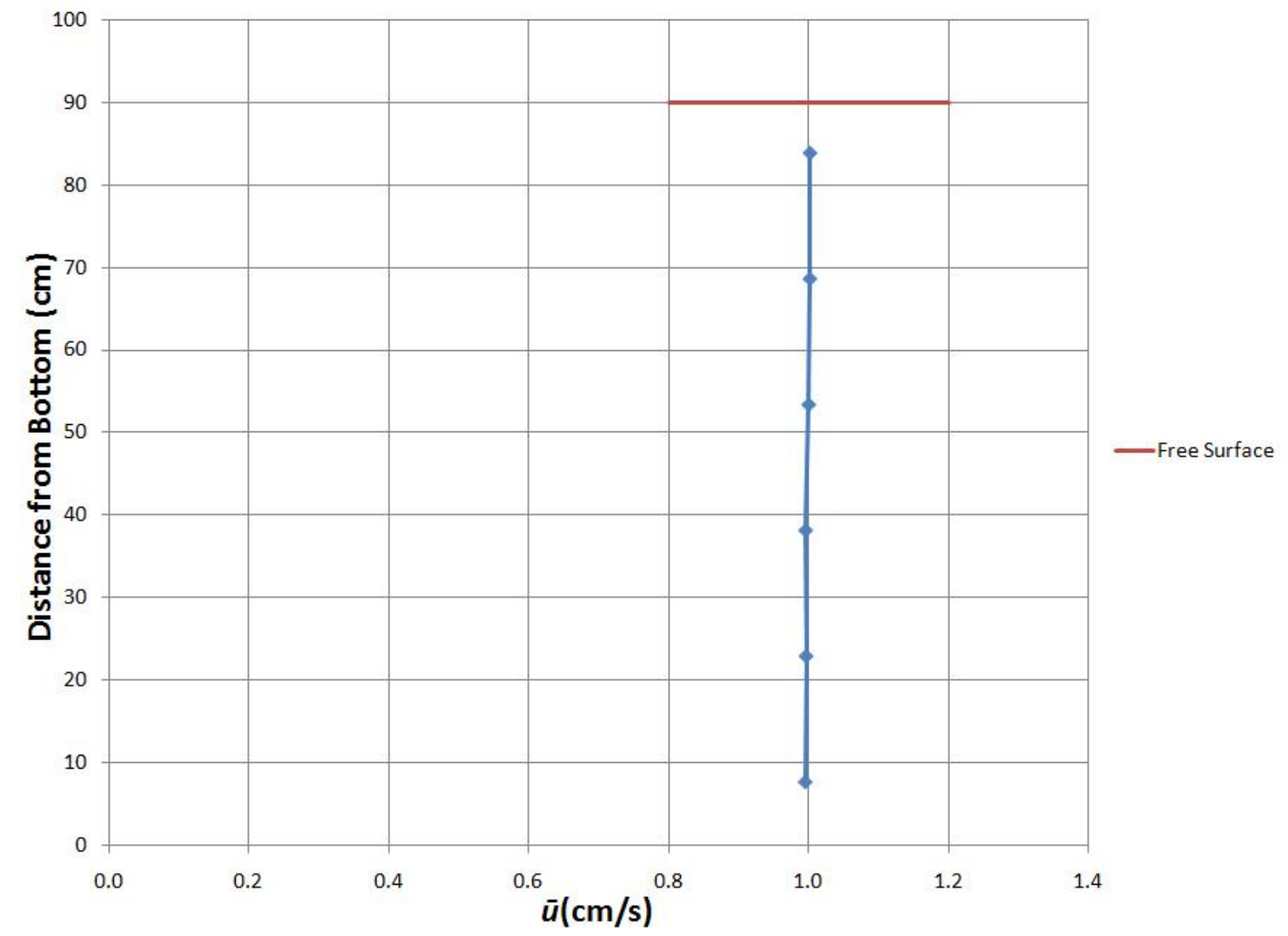

Figure 36: Vertical Profile of Mean Streamwise Velocity (Rumberg, 2004)

Figure 36 shows a vertical profile of mean streamwise velocity measured by Rumberg (2004). This profile is measured approximately at the center of quiescent zone B4 and contains 6 measuring points. The first point is located approximately $7.6 \mathrm{~cm}$ from the bottom of the 
quiescent zone with each subsequent measurement taken roughly $15 \mathrm{~cm}$ thereafter. Rumberg measured an average flow rate of approximately $9.0 \times 10^{-3}$ through the raceway system.

Like Huggins, Rumberg (2004) used SonTek's FlowTracker ADV to measure the mean velocities in the quiescent and fish zones at Dogwood Lake. Each mean velocity was sampled at $1 \mathrm{~Hz}$ and averaged over 10 seconds. Rumberg calculated an average Reynolds number of approximately $7.6 \times 10^{3}$. This Reynolds number is small, showing that the flow is unstable and almost turbulent $(\operatorname{Re}>8000)$ and a 10 second averaging time may be inadequate to capture accurate mean velocities. A running average measured by Rumberg (2004) in quiescent zone B4 can be seen in Figure 37 and shows that a 10 second averaging time is inadequate for measuring all mean velocities. Figure 37 shows an averaging time of approximately 200 seconds as the minimum averaging time required to capturing accurate mean velocities.

A comparison of Figure 36 to Figure 34 shows that Rumberg's vertical profile has fewer measuring points and the separation between each measurement point is approximately 6 times as large. Also, the mean velocities measured by Rumberg are not accurate due to a short averaging time of 10 seconds.

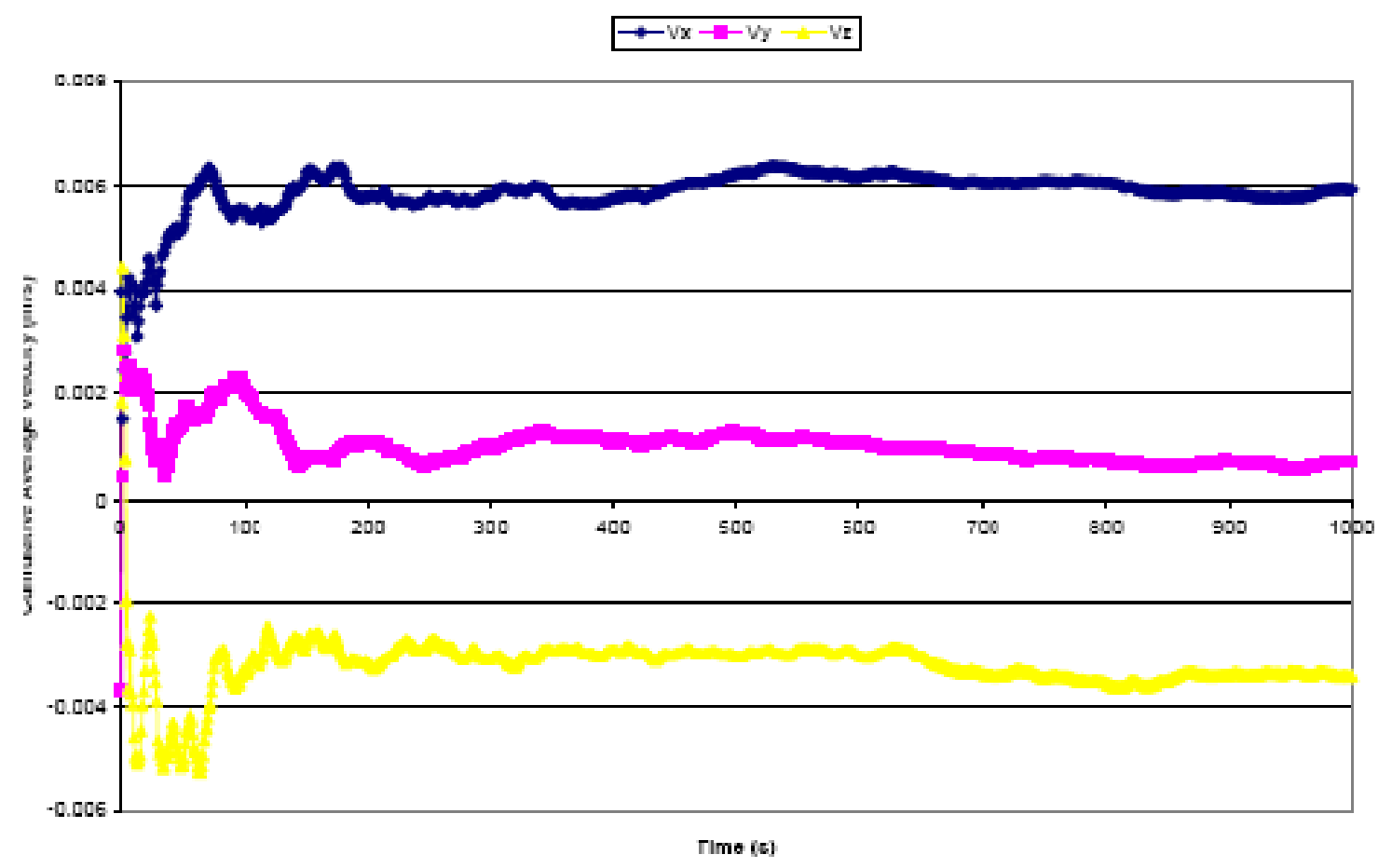

Figure 37: Running Average all Three Velocity Components (Rumberg, 2004) 


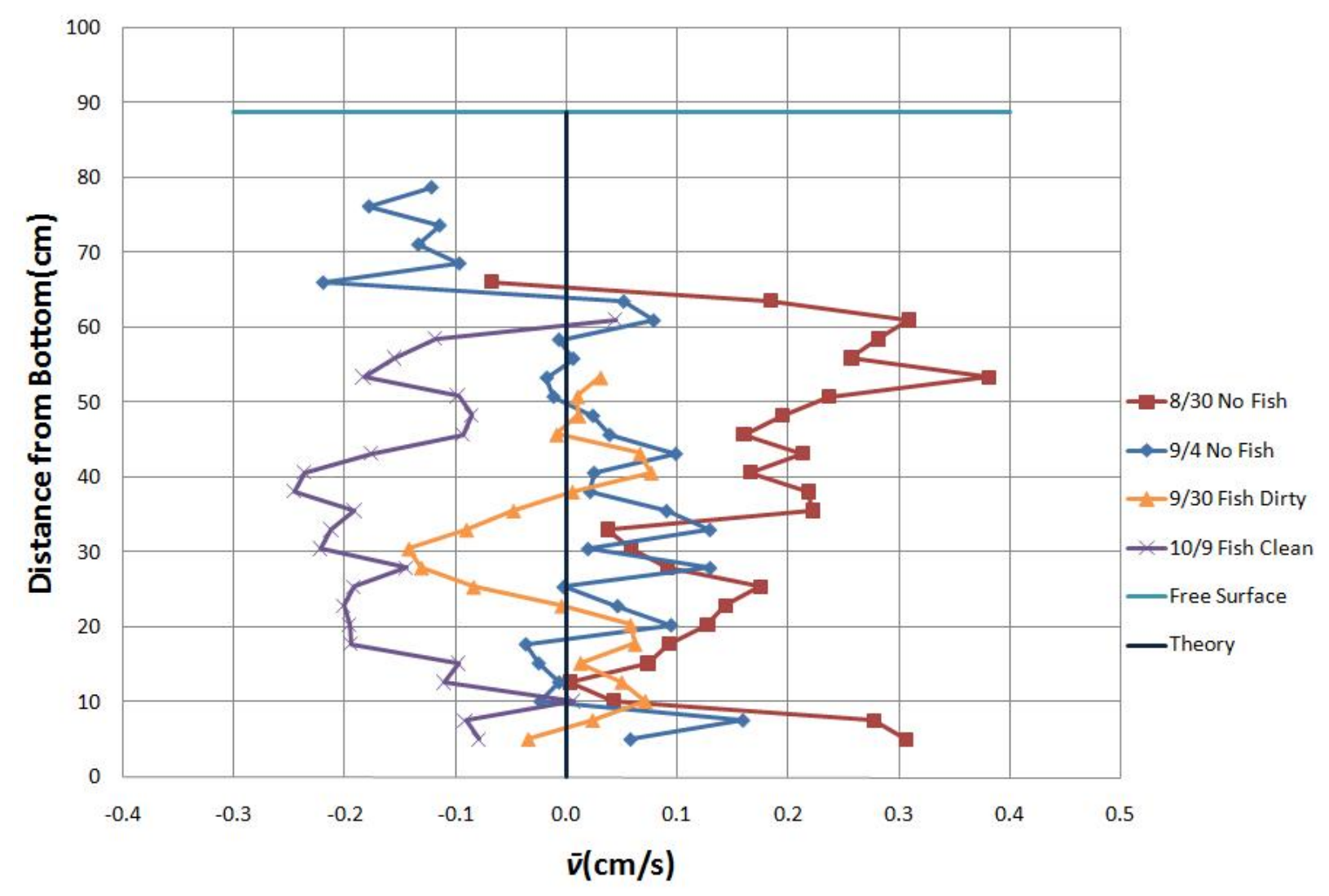

Figure 38: Vertical Profiles of Mean Transverse Velocity

Figure 38 shows vertical profiles of the mean transverse velocity corresponding to Figures 26-29.

The measured profiles in Figure 38 do not have a constant zero mean transverse velocity profile as two-dimensional flow should (discussed in Section 4.5.0). This may be the result of screen blockage, causing the flow to divert in a transverse path. Again, other possible causes of discrepancy between the measured and two-dimensional theoretical profiles are the smoothness of the raceway system, and disturbances (upstream fish and screen, wind shear).

In order to determine if the measured mean transverse velocity actually exists, dye was injected in to the beginning of quiescent zones A4 and B4. The injected dye traveled to the left of the quiescent zones and then to the right near the end to the exit the weir. This test validates that transverse flow actually exists in the quiescent zones.

It should be observed that differences between profiles measured in the same quiescent zone (August 30 and September 4) vary drastically when approaching the free surface. This may 
be due to variations in daily disturbances and factors such as wind shear, rotational sensitivity of the $\mathrm{ADV}$, misalignments, and cleaning of the screens prior to taking measurements. It was noted that wind speeds were high on August 30 (Table 8), and may cause daily differences in mean streamwise velocities near the free surface.

Rotational sensitivity can alter the values of the mean transverse velocities. If the ADV probe is aligned differently on different days, it will cause different values of mean transverse velocity. Marks placed on the traversing mechanism and ADV probe help limit daily misalignment, but do not guarantee $100 \%$ confidence. It is estimated that a maximum of $\pm 1^{\circ}$ of misalignment could occur with respect to rotation around the $z$-axis. Table 7 shows that a misalignment of $\pm 1^{\circ}$ will increase the mean transverse velocity by $0.59 \mathrm{~cm} / \mathrm{s}$. An increase of $0.59 \mathrm{~cm} / \mathrm{s}$ will cause a drastic error with respect to the magnitudes of mean transverse velocity measured in Figure 38. This shows that angular sensitivity is a very large factor when measuring mean transverse velocities.

Uncertainty of alignment of the traversing mechanism (effects shown Tables 4-6) can also alter the mean transverse velocities. An average error of $\pm 0.060 \mathrm{~cm} / \mathrm{s}$ is found due to misalignments affecting the mean transverse velocity. In some locations the difference in profiles are on the same order of magnitude as this error, therefore this error must be considered when determining discrepancies between measured profiles.

Comparing the profiles measured on August 30 and September 4 to those measured on October 9 , it can be concluded that the presence of fish, different screen types, rotational sensitivity, and error resulting from alignment uncertainty will alter the mean transverse velocity profiles. When comparing profiles from September 30 to those measured on October 9, it can be concluded that an unclean screen will alter the mean transverse velocity profile. 


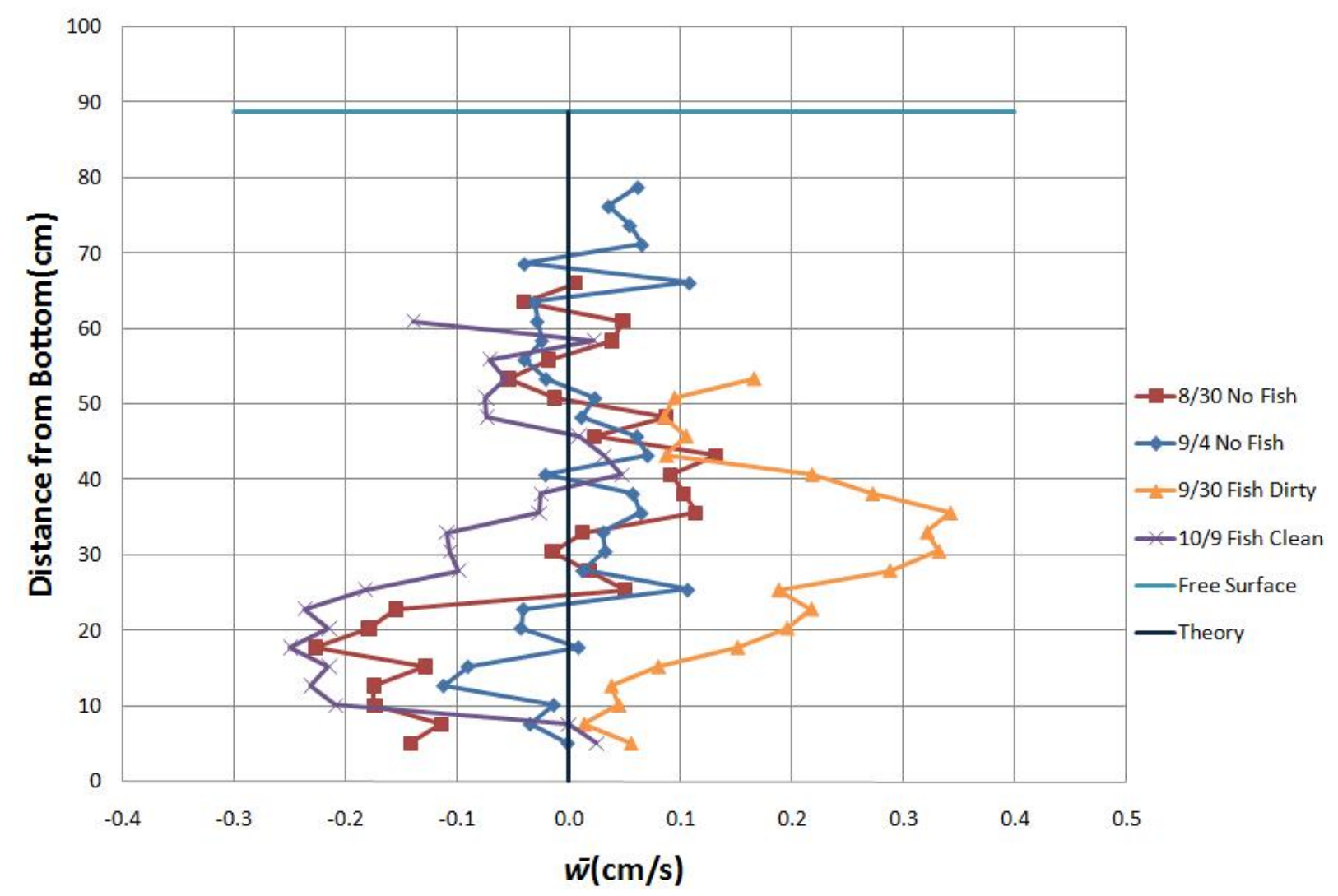

Figure 39: Vertical Profiles of Mean Vertical Velocity

Figure 39 shows vertical profiles of the mean vertical velocity corresponding to Figures 26-29.

It should be noticed that all profiles vary from the theoretical profile. For twodimensional, fully developed, open channel flow the mean vertical velocity should be zero throughout the entire channel. Profiles measured on August 30, September 4, and October 9 have similar shapes. Since these profiles are similar, the presence of fish and different screen types may not be causing drastic affects on the shape of the mean vertical velocity profiles in Figure 39. The upstream presence of the screen and fish may be a factor in causing discrepancies between the theoretical and measured profiles. Comparing the previously mentioned measured profiles to the profile measured on September 30 shows that a dirty screen significantly disturbs the mean vertical velocity profile. The consistency of the dirty screen causing large differences in measured profiles is steady throughout the entire study. This supports the idea that this variation between profiles actually exists, and is not just a factor of angular sensitivity and misalignment. Misalignment due to the traversing mechanism (average 
of $\pm 0.050 \mathrm{~cm} / \mathrm{s}$ for mean transverse velocities) and rotational sensitivity $(-0.21 \mathrm{~cm} / \mathrm{s}$ for mean transverse velocities with $\pm 1^{\circ}$ misalignment) are factors, but not the only factors contributing to the differences in profiles measured on different days.

\subsubsection{Transverse Profiles of Mean Velocity}

For each transverse profile of the mean velocity, the starting measurement point is located approximately $7.7 \mathrm{~cm}$ from the right side of the quiescent zone (when looking downstream) and $7.7 \mathrm{~cm}$ from the bottom. The profiles end at varying distances from the left side of the quiescent zone due to occasional problems with the ADV (as mentioned in Section 8.3.0) and varying dimensions of the different quiescent zones. The measurement points of the transverse profiles are separated by approximately $2.5 \mathrm{~cm}$.

For each transverse profile, the black curve represents the theoretical/empirical fully developed, turbulent open channel mean transverse velocity profile approximated using Equation 34 from both sides of the quiescent zone. Equation 34 causes an unrealistic cusp at the center of the quiescent zone for the transverse profile of mean streamwise velocity. This cusp shows that viscous forces are present in the center which is unrealistic for two-dimensional, fully developed, turbulent, open channel flow. The horizontal brown lines located at 0 and $91 \mathrm{~cm}$ on the y-axis represent the right and left sides of the quiescent zone, respectively. 


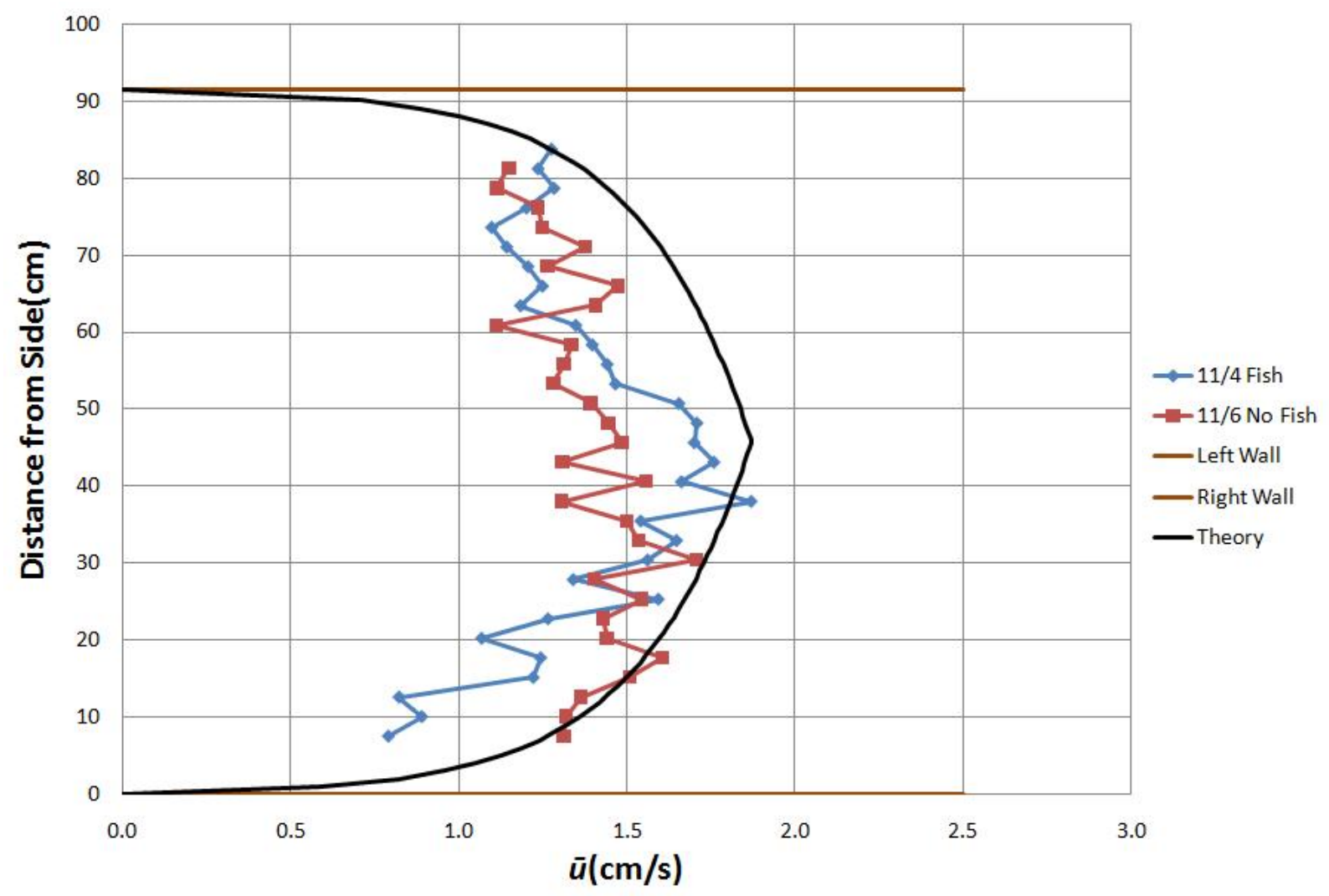

Figure 40: Transverse Profiles of Mean Streamwise Velocity

Figure 40 shows transverse profiles of the mean streamwise velocity corresponding to Figures 30-31.

The theoretical/empirical curve in Figure 40 shows that the width of the channel is too narrow to permit two-dimensional flow near the center of the quiescent zone. Considering the previous observation, it is expected that the measured profiles will not contain two-dimensional characteristics.

As expected, the two measured profiles do not match the theoretical/empirical profile. The shape of the profile measured on November 4 does have a similar shape as the theoretical/empirical, but varies erratically and has a smaller magnitude. The profile measured on November 6 is more constant throughout the width of the quiescent zone than the profile measured on November 4. The constant shape of the profile measured on November 6 is most likely due to having a less debris obstructing the large screen than to the small screen. 
A comparison of the two measured profiles shows that the presence of fish and different screen types can affect the transverse profile of the mean streamwise velocities and may also be the cause of discrepancies between the theoretical/empirical and measured profiles in Figure 40.

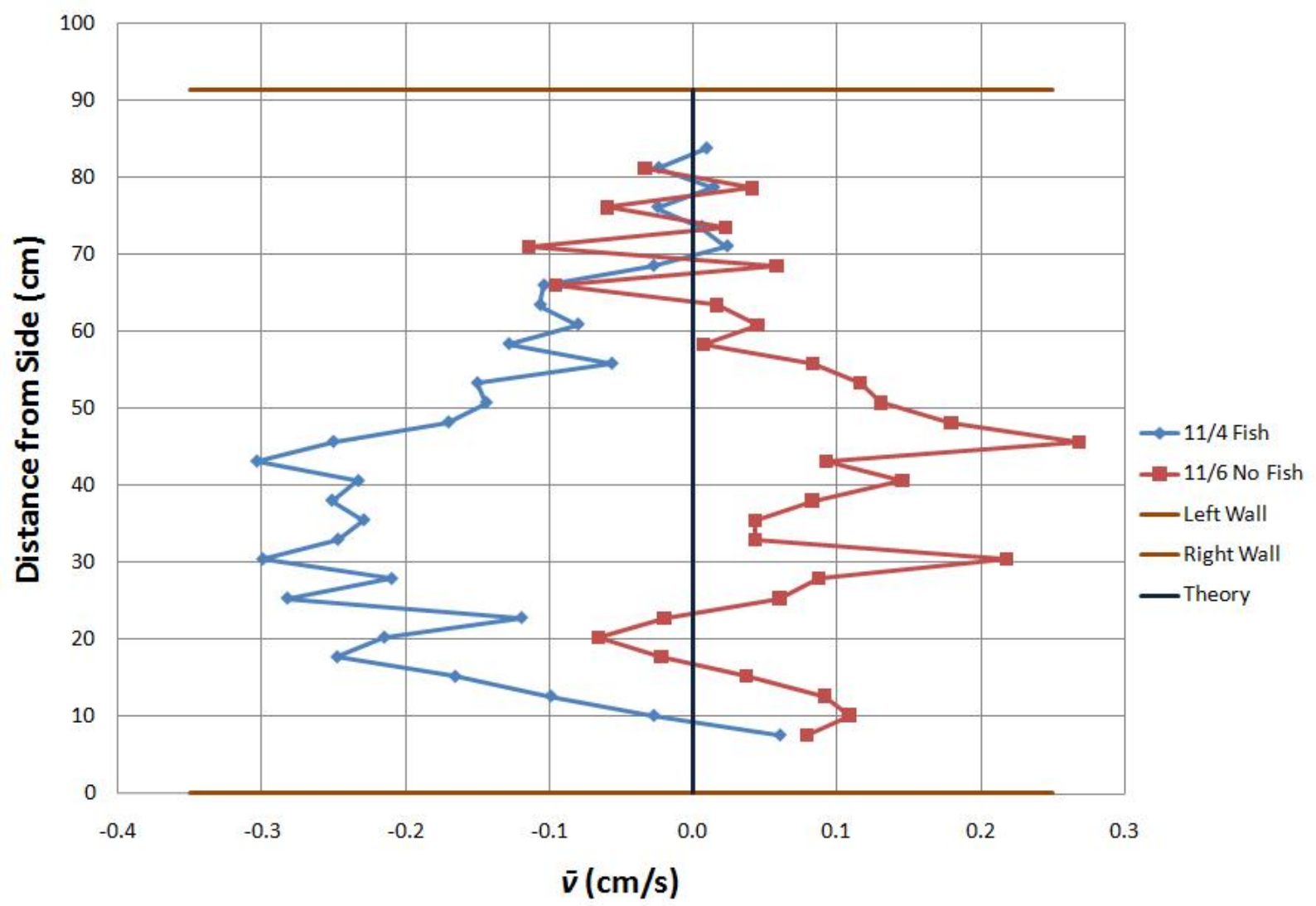

Figure 41: Transverse Profiles of Mean Transverse Velocity

Figure 41 shows transverse profiles of the mean transverse velocity corresponding to Figures 30 31.

The two measured profiles do not agree with the theoretical profile. The mean transverse velocity should be zero throughout the entire channel for the flow to be considered twodimensional and fully developed. The two measured profiles have similar magnitudes of mean transverse velocity, but act in different directions. As the measured profiles approach the left wall (looking downstream), they appear to possess similar direction and magnitude. This may be an indication that the left wall shear stress is causing a larger effect on the flow than the right. This could be due to a larger growth of algae on the left side wall than the right. The buildup of algae on the side walls could be causing viscous forces to affect the flow further from the wall 
than a wall with no algae growth. An increase in viscous forces could outweigh the effects of turbulence and allow for random fluctuations to be ignored, allowing for more consistency in the profiles in Figure 41 near the left wall. The difference in direction between the measured profiles may be a result of upstream presence of fish, different screen types, and debris obstructing the screen. The error resulting from misalignment and rotational sensitivity will also have effect on the differences between measurements in Figure 41.

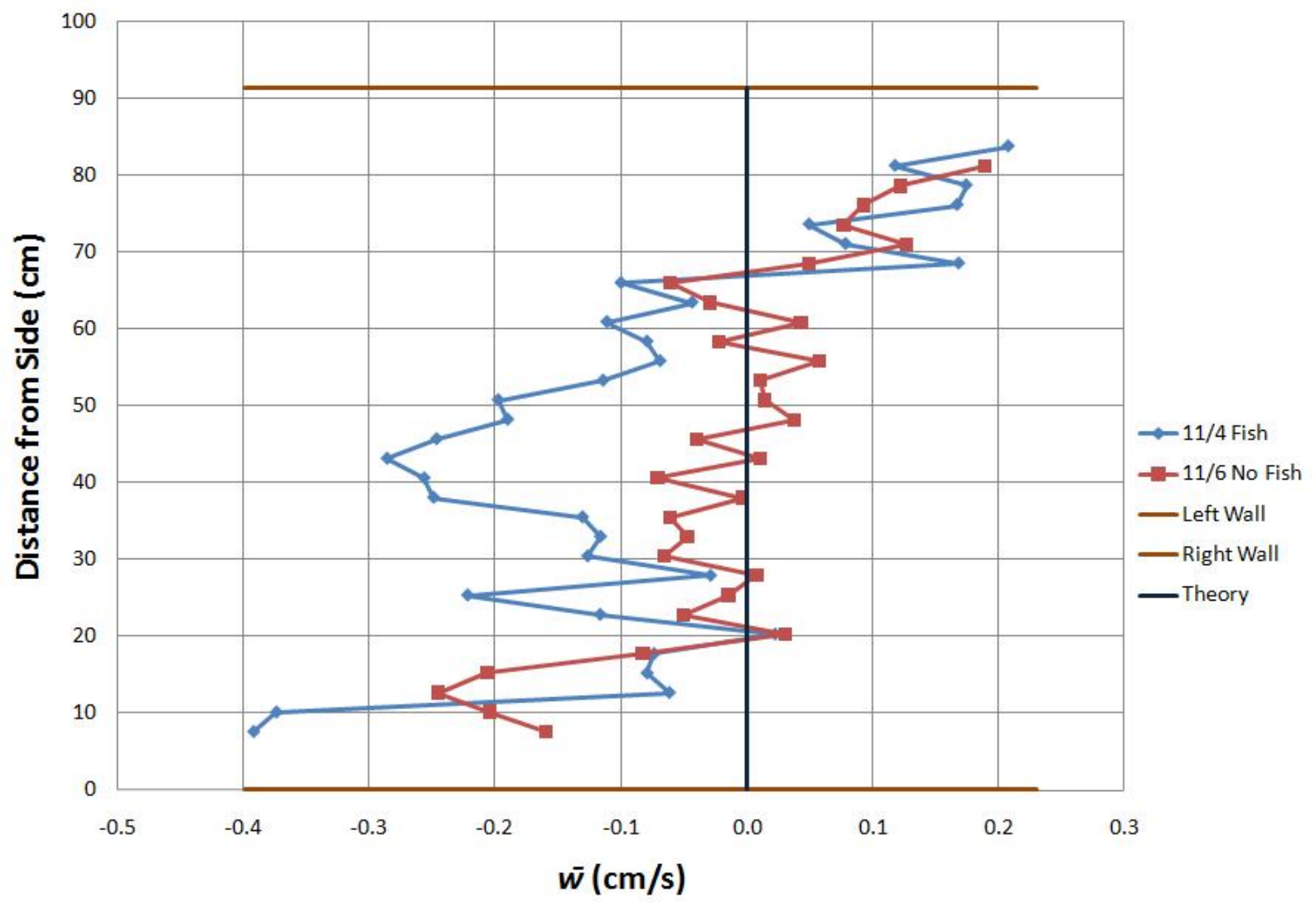

Figure 42: Transverse Profiles of Mean Vertical Velocity

Figure 42 shows transverse profiles of the mean vertical velocity corresponding to Figures $30-31$.

The measured profiles differ from the theoretical profile. The mean vertical velocity should be zero throughout the entire channel for two-dimensional, fully developed, open channel flow. The measured profile on November 6 appears to average zero near the center of the quiescent zone. However, this is not the case for the profile measured on November 4. This comparison shows that the upstream presence of fish and/or a small screen is causing larger 
discrepancies between the theoretical profile than zero fish upstream and a large screen. Again, rotational sensitivity and misalignment cannot be ignored.

\subsubsection{Streamwise Mean Velocity Profiles}

For each streamwise profile of the mean velocity, the starting measurement point is located approximately $58 \mathrm{~cm}$ from the beginning of the quiescent zone and $7.7 \mathrm{~cm}$ from the bottom. The measurement points are separated by approximately $2.5 \mathrm{~cm}$. The two profiles end at varying distances from the end of the quiescent zone due to occasional problems with the ADV (discussed in Section 8.3.0). The horizontal brown line represents the end of the quiescent zone for each streamwise profile.

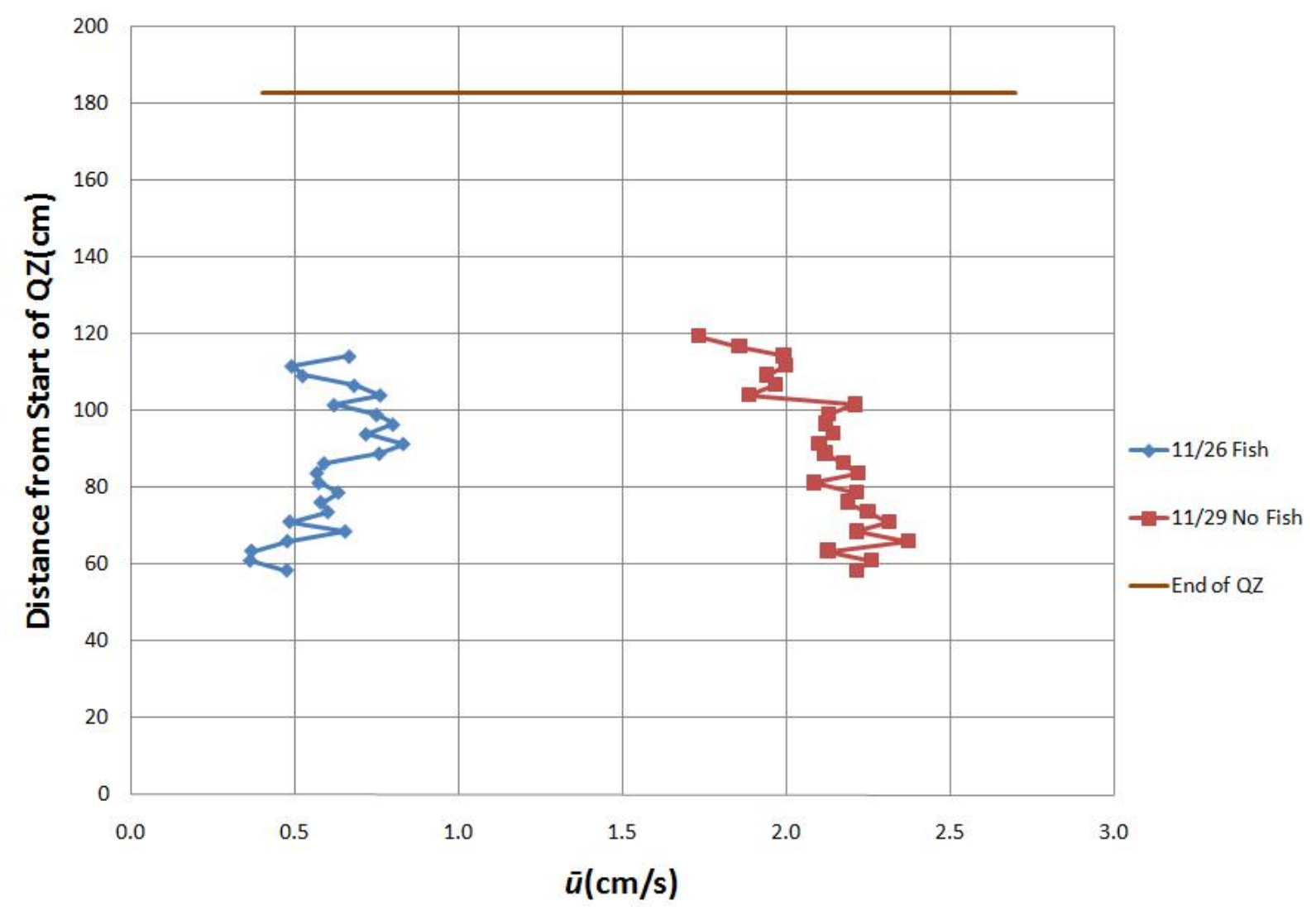

Figure 43: Streamwise Profiles of Mean Streamwise Velocity

Figure 43 shows the streamwise profile of the mean streamwise velocity corresponding to Figures 32-33.

In order for the flow to be fully developed, the mean velocities must be constant when traversing in the streamwise direction at the same distance from the bottom. Neither streamwise 
profile shows a constant mean streamwise velocity when traversing in the streamwise direction. This supports the conclusion that the flow is not fully developed.

When comparing the two measured profiles in Figure 43, the large difference in flow rates should be noted (Table 8 ). This is likely the cause of the rather large difference in magnitudes of the mean streamwise velocities.

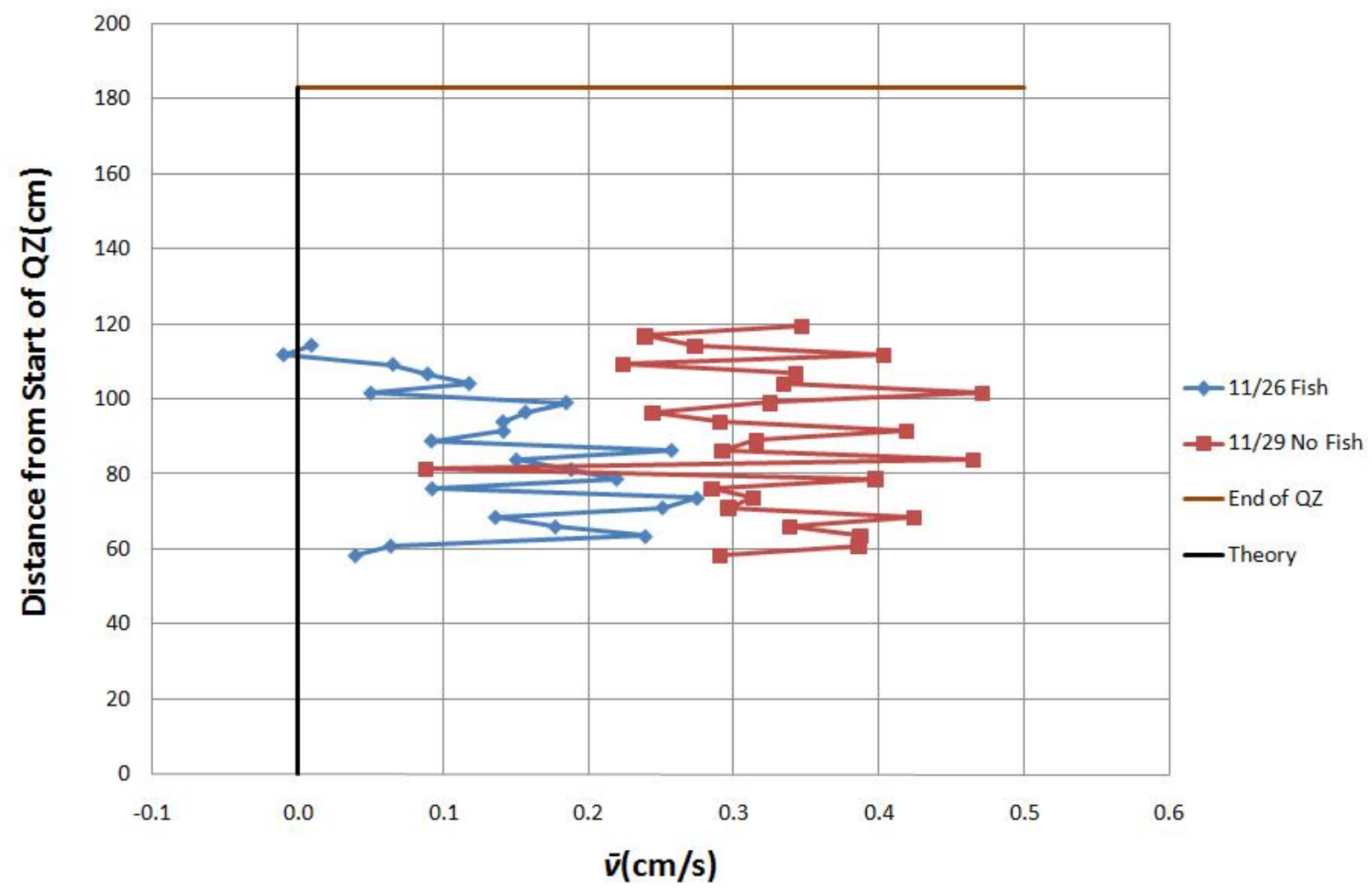

Figure 44: Streamwise Profiles of Mean Transverse Velocity

Figure 44 shows the streamwise profile of the mean transverse velocity corresponding to Figures 32-33.

The measured profiles in Figure 44 do not correspond to the theoretical profile. For twodimensional, fully developed, open channel flow the mean transverse velocity should be zero throughout the entire channel. The measured profiles in Figure 44 show a varying mean transverse velocity in the streamwise direction. This variation of mean transverse velocity may be a result of debris on the screen causing the flow to divert to a transverse path. Upstream fish and different screen types may also be the cause of differences in the two measured profiles. 
Errors from rotational sensitivity and misalignment will have an effect on profiles and should not be ignored.

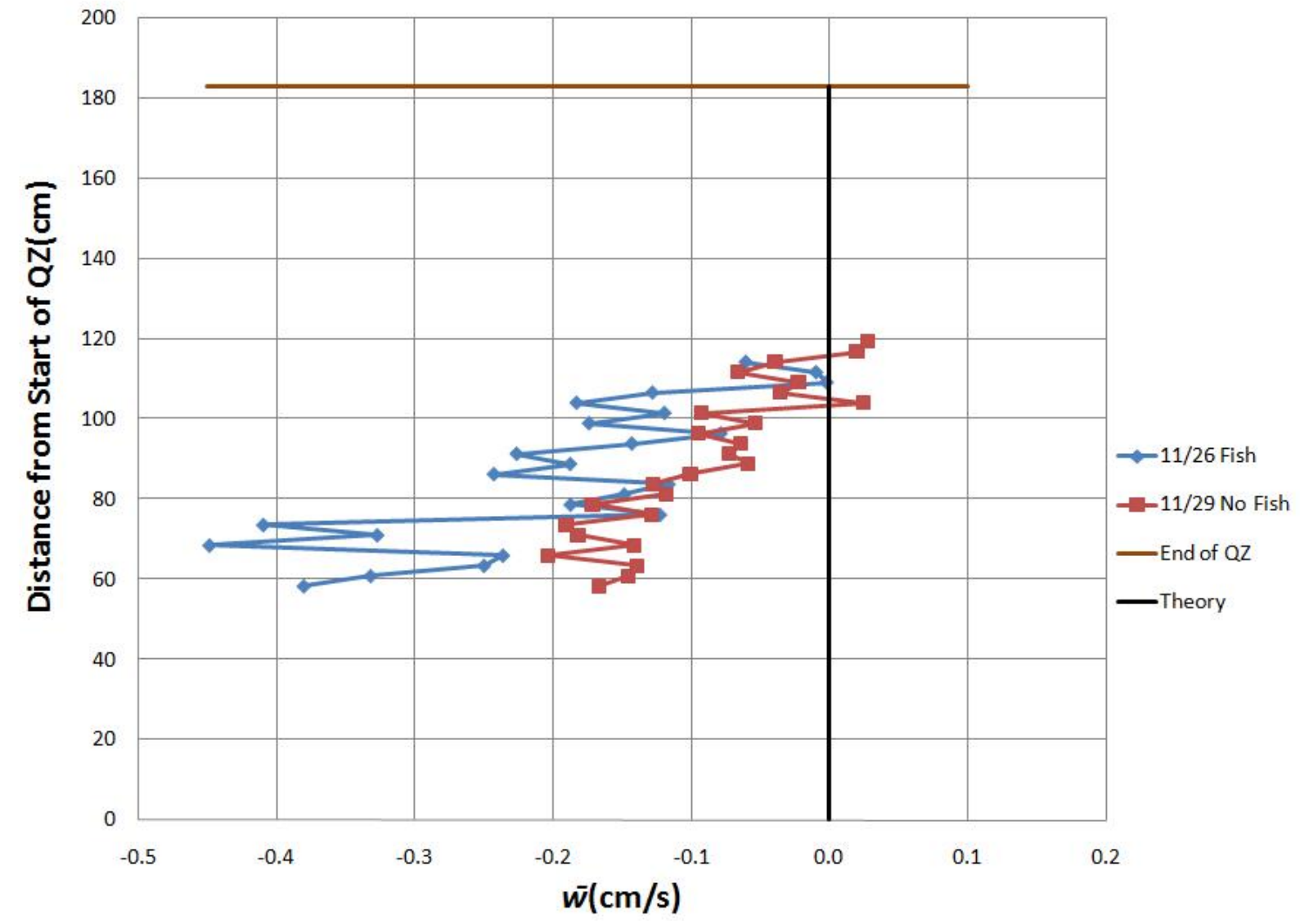

Figure 45: Streamwise Profiles of Mean Vertical Velocity

Figure 45 shows the streamwise profile of the mean vertical velocity corresponding to Figures 32-33.

The measured profiles in Figure 45 do not correspond to the two-dimensional, fully developed, turbulent open channel flow. However, as the flow approaches the weir, the velocities are expected to change direction and begin to move upward toward the weir. This upward movement of the mean vertical velocities is present in Figure 45 as the distance from the weir decreases. The difference between the two measured profiles may be the result of upstream disturbances such as different screen types and the presence of fish. As mentioned previously, errors resulting from misalignment and rotational sensitivity cannot be ignored. 


\subsubsection{Vertical Profiles of Root Mean Square (RMS) Velocities}

The following profiles are vertical root mean square (RMS) velocity profiles that correspond to measurements taken in Figures 26-29. The velocity fluctuations are averaged over a 360 second time interval. The black curves in Figures 46 and 49 represent theoretical/empirical approximations of vertical profiles of $u_{R M S}$ and $w_{R M S}$ (Equations 16-17).

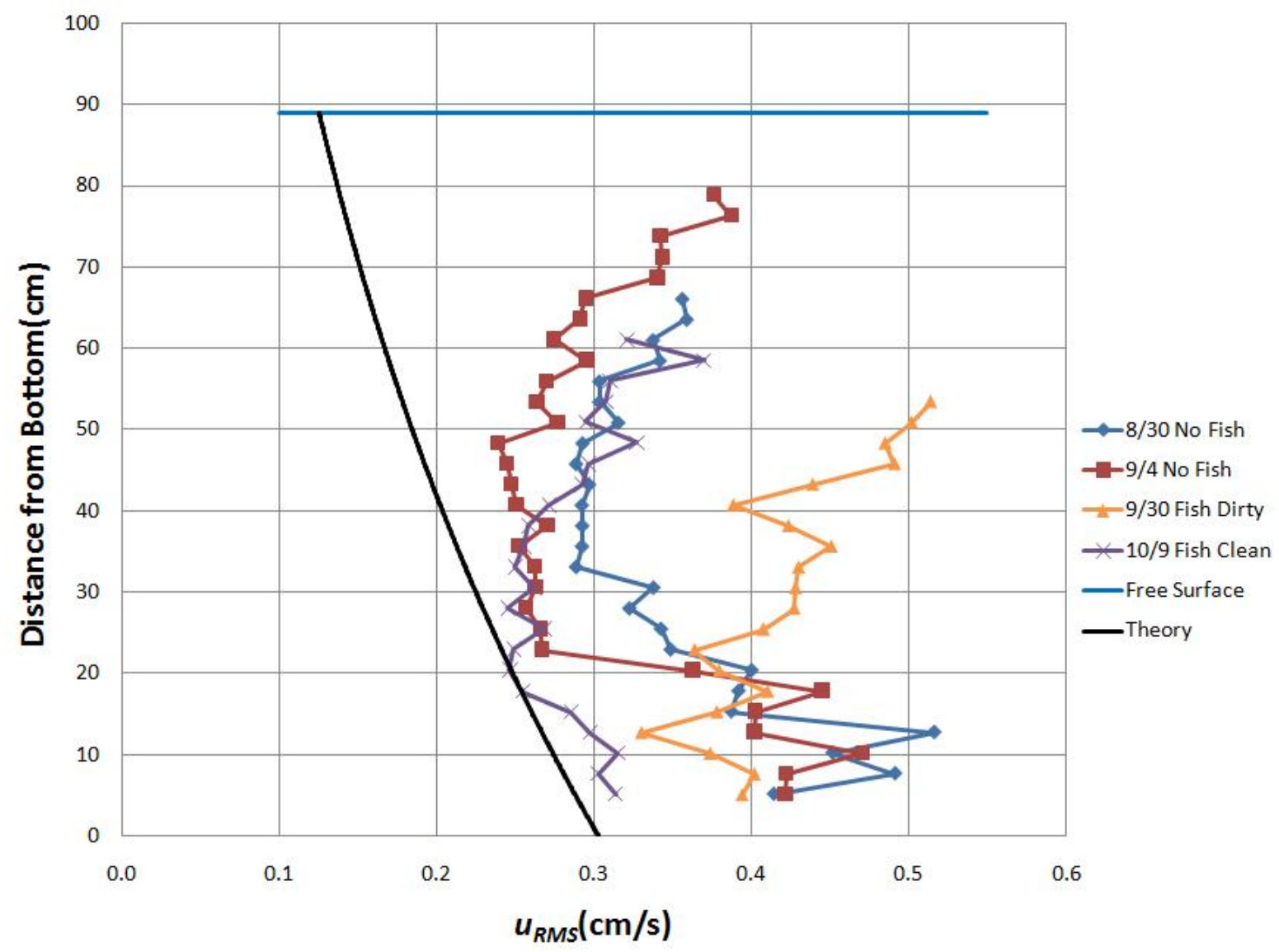

Figure 46: Root Mean Square Streamwise Velocity Profiles

Figure 46 shows the vertical profile of $u_{R M S}$ corresponding to Figures 26-29.

In Figure 46 the general shape of the measured $u_{R M S}$ vertical profile (excluding measurements taken on September 30) is roughly the same as the theoretical/empirical approximation (given by Equation 16). The profiles begin to vary from the theoretical/empirical profile as they approach the free surface due to disturbances (most likely wind shear) which affect the flow. All measured profiles (excluding measurements taken on September 30) in Figure 46 have relatively high magnitudes of RMS between 5 and $15 \mathrm{~cm}$ from the bottom of the quiescent zone, decrease in magnitude toward the center of the quiescent zone, and then increase 
in magnitude towards the free surface. It should be noted that Equation 16 does not account for free surface disturbances and can be used to approximate $u_{R M S}$ in the turbulent layer (discussed in Section 4.5.0).

When comparing the profiles measured on August 30 and September 4 to the profile measured on October 9, it can be seen that the presence of fish and a small screen upstream creates lower magnitudes of fluctuations in the streamwise direction closer to the bottom of the quiescent zone when compared to zero fish present and a large screen upstream. If it is assumed that the fish will always increase fluctuations in all directions, then the reduction in magnitude of the fluctuations must be attributed to the screen. The previous observation can be thought of as larger eddies "cut" into smaller eddies from the small screen. This allows for a conclusion that the fish will not increase fluctuations near the bottom as long as a small screen is placed upstream of the quiescent zone. A comparison of measurements taken on September 30 and October 9 indicates that cleaning the small screen should reduce the magnitudes of the fluctuations in the streamwise direction.

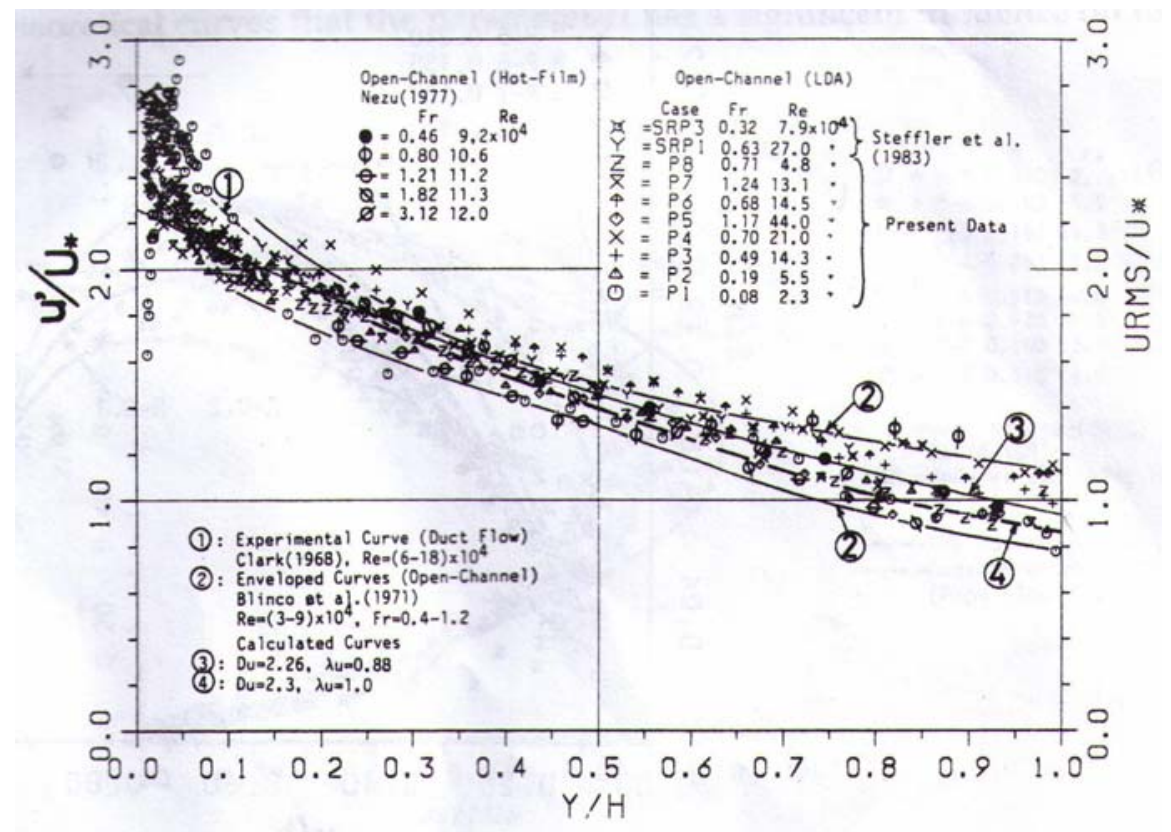

Figure 47: Root Mean Square Streamwise Velocity Profiles (Nezu and Rodi, 1986)

Figure 47 shows a plot of non-dimensional streamwise RMS versus non-dimensional distance from the bottom (Nezu and Rodi, 1986). Several measurements are plotted at different Reynolds numbers as well as theoretical/empirical curves calculated from Equation 16. 
Profiles in Figure 47 do not show much discrepancy between the approximated theoretical/empirical results in the turbulent layer (discussed in Section 4.5.0). This is a result of having the proper channel geometry, and the ability of limiting disturbances by measuring velocities in a flume where theoretical/empirical characteristics may be achieved.

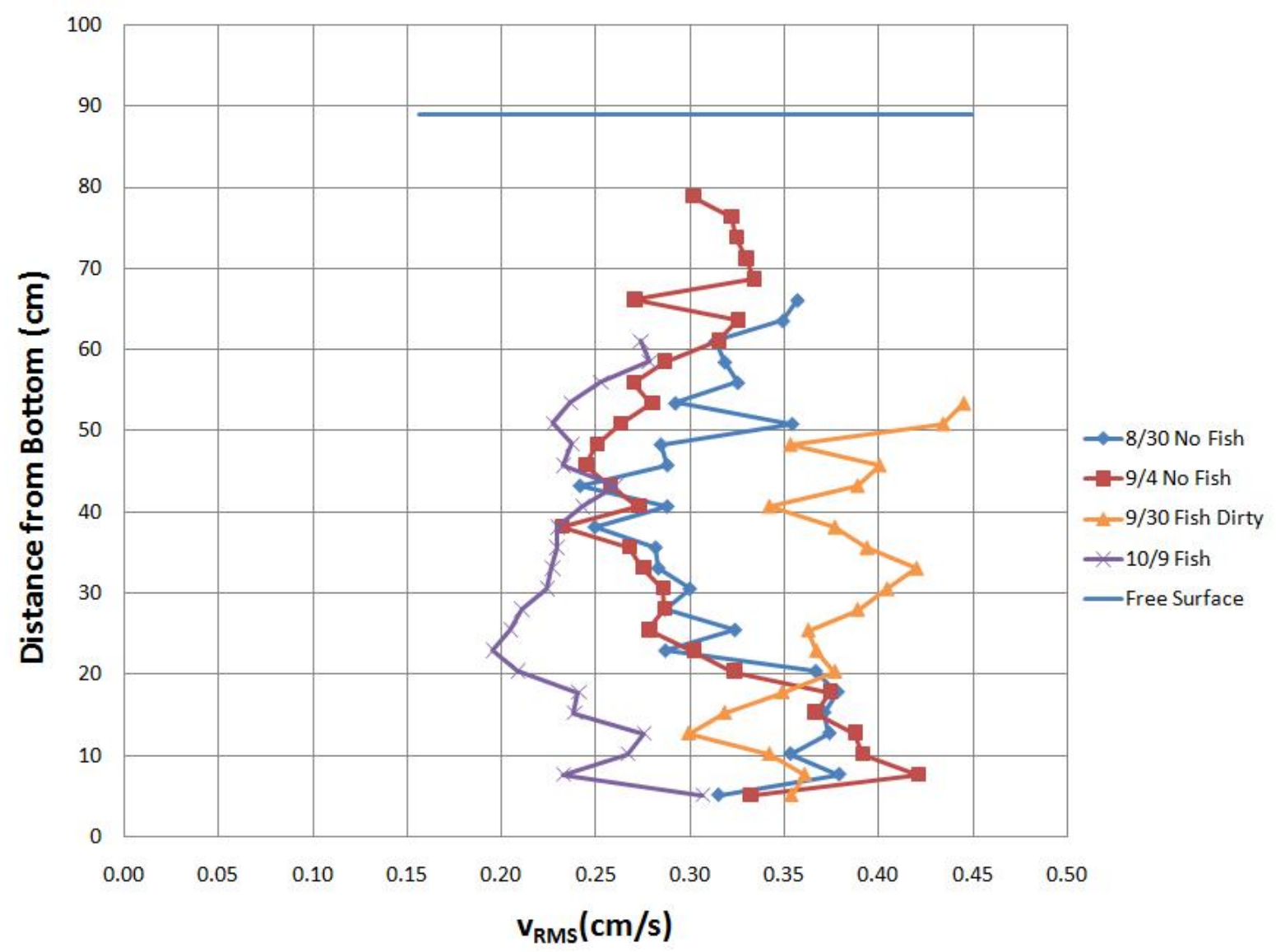

Figure 48: Root Mean Square Transverse Velocity Profiles

Figure 48 shows the vertical profile of $v_{R M S}$ corresponding to Figures 26-29. There is no theoretical/empirical profile available for the vertical profile of transverse RMS velocity.

The $v_{R M S}$ profiles measured on August 30 and September 4 are similar in shape. The measured RMS profiles in Figure 48 have larger values of RMS near the bottom, reduce in magnitude towards the center, and then increase in magnitude closer to the free surface. This may be due to free surface disturbances. Comparing values of $v_{R M S}$ of profiles measured on August 30 and September 4 to the profile measured on October 9 shows that different screen types and fish upstream may affect the vertical profile of $v_{R M S}$. 
Due to a large difference in $v_{R M S}$ in profiles measured on September 30 and October 9, it may be concluded that a debris blocked screen is most likely the major contributor to differences of the vertical $v_{R M S}$ profiles. In addition, the magnitude of the fluctuations in the transverse direction is dependent on the type of screen and the debris it contains and not the presence of fish. A dirty screen causes the magnitude of transverse fluctuations to increase with respect to a clean screen, whereas a large screen causes the magnitude of transverse fluctuations to increase with respect to a small screen.

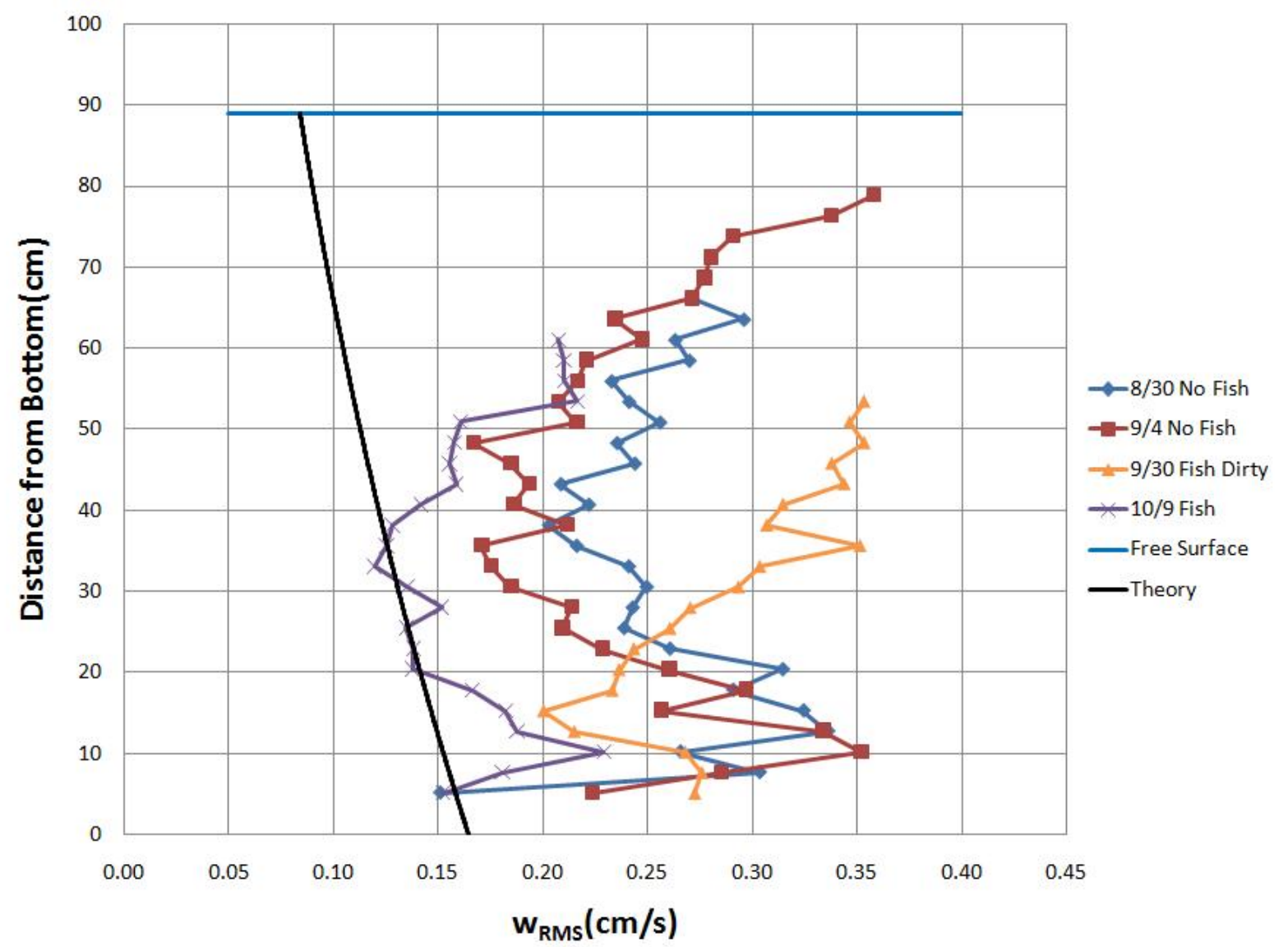

Figure 49: Root Mean Square Vertical Velocity Profiles

Figure 49 shows the vertical profile of $w_{R M S}$ corresponding to Figures 26-29.

The $w_{R M S}$ measured profiles in Figure 49 show a resemblance to the theoretical/empirical approximation (Equation 17) because they both decrease in magnitude toward the center of the quiescent zone, but vary from the theoretical/empirical profile erratically. It should be noted that the theoretical/empirical profile in Figure 49 is plotted from the bottom surface to the free surface for simplicity, but is only valid in the turbulent layer (discussed in Section 4.5.0). 
As seen in Figure 46, all measured profiles (excluding measurements taken on September 30) in Figure 49 have relatively high magnitudes of RMS between 5 and $15 \mathrm{~cm}$ from the bottom of the quiescent zone, decrease in magnitude toward the center of the quiescent zone, and then increase in magnitude towards the free surface. The increase towards the free surface may be due to free surface disturbances such as wind shear.

When comparing profiles measured on August 30 and September 4 to profiles measured on October 9, it should be noted that a smaller screen may also be creating smaller magnitudes of $w_{R M S}$ with respect to a large screen. Comparing profiles measured on September 30 and October 9 in Figure 49, it is observed that a dirty small screen increases the magnitude of vertical fluctuations with respect to a small clean screen.

\subsubsection{Turbulence Intensity Profiles}

The following profiles are turbulence intensity (TI) profiles (calculated using Equations 14-15) that correspond to measurements taken in Figures 26-33. The fluctuations are averaged over a 360 second time interval. Turbulence intensity profiles in Figures 50-52 account for fluctuations in all directions. A theoretical/empirical approximation for turbulence intensities is not available. 


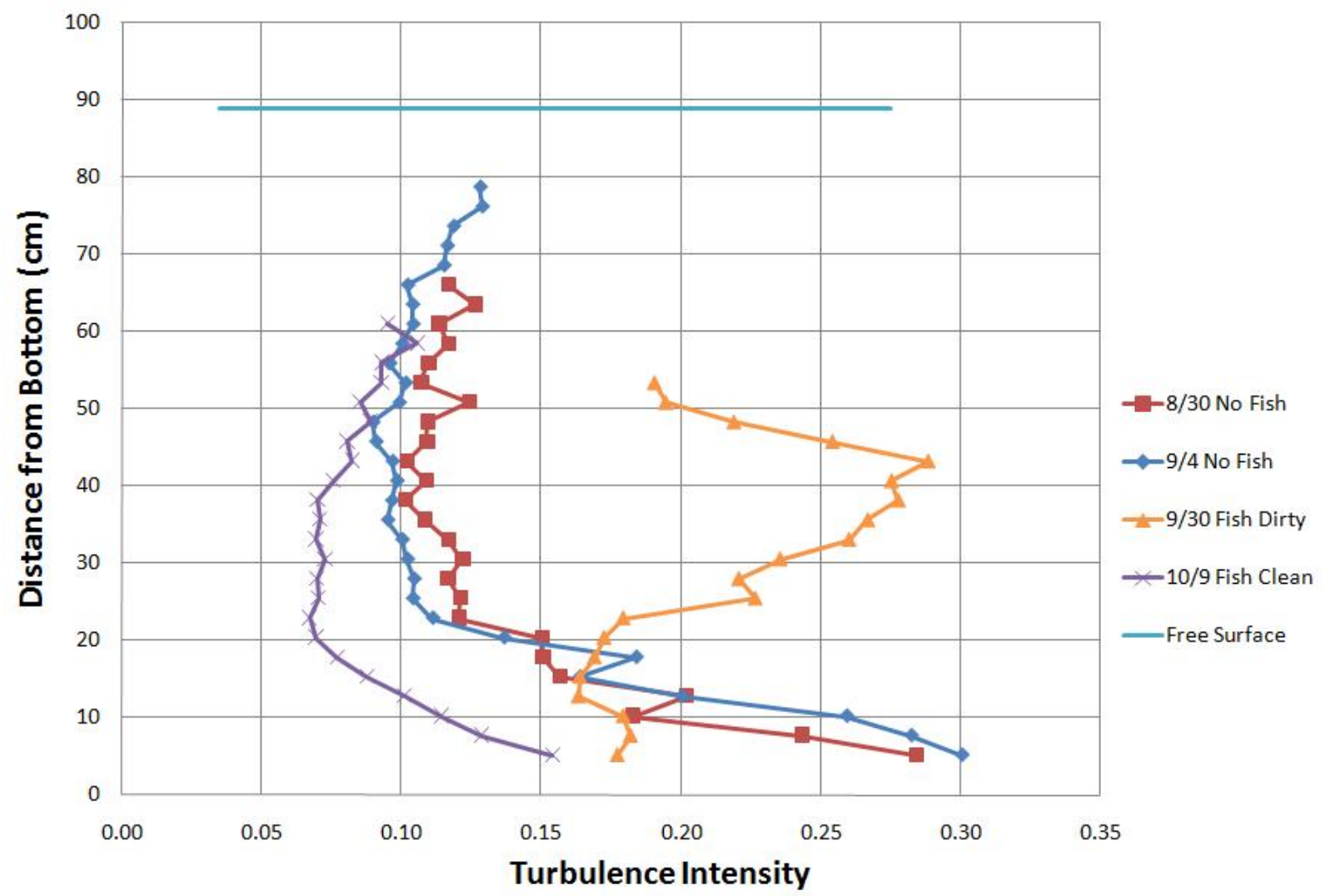

Figure 50: Vertical Profiles of Turbulence Intensities

Figure 50 represents the profile of turbulence intensities corresponding to Figures 26-29.

After reviewing Figures 46, 48, and 49, it is expected that the September 30 profile would have the largest magnitudes of fluctuation, followed by August 30, September 4, and October 9 profiles. The previous observation supports the idea that the small screen reduces the magnitude of turbulence. The small screen breaks the larger eddies into smaller ones, causing a lower magnitude of turbulence. Small meshed screens are also used in wind tunnels to reduce the level of turbulence, proving that this result exists in other fluids.

Figure 50 also supports the assumption that a dirty screen will increase the magnitude of turbulence when compared to a clean screen. A conclusion can be drawn that the magnitude of turbulence in the quiescent zone is dependent mostly on the screen type and the presence of debris blocking the screen, and while approaching the free surface, the magnitude of TI is dependent on the free surface disturbances. 


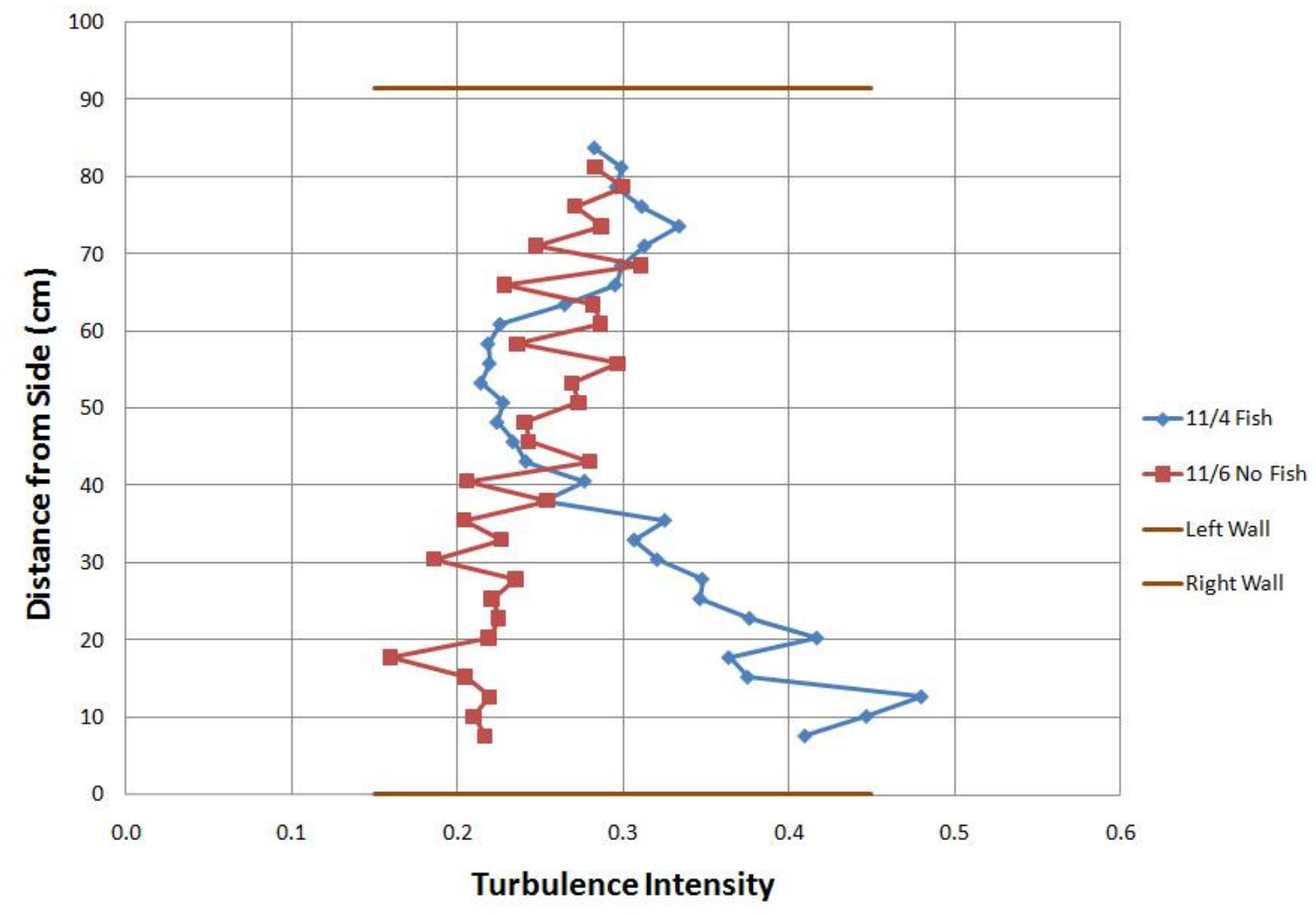

Figure 51: Transverse Profile of Turbulence Intensities

Figure 51 represents the transverse profile of turbulence intensities corresponding to Figures 30-31.

The profile measured on November 4 has a less consistent turbulence intensity profile than the profile measured on November 6 . The November 4 profile has a small screen upstream, while the November 6 has a large screen upstream. As mentioned in Section 8.1.0, a small screen is more difficult to clean due to the debris becoming lodged between the plastic square mesh and the aluminum diamond section (Figure 24). Debris may be responsible for the greater variation measured on November 4. It may also be responsible for the larger magnitudes of turbulence near the right side of the quiescent zone for the profile measured on November 4.

The concept of the small screen breaking the larger eddies into smaller ones may still be applied, but debris blocking the screen will cause the magnitudes of turbulence to increase. It can be concluded that debris blocking the small screen may counter the reduction of turbulence that a small screen creates. 


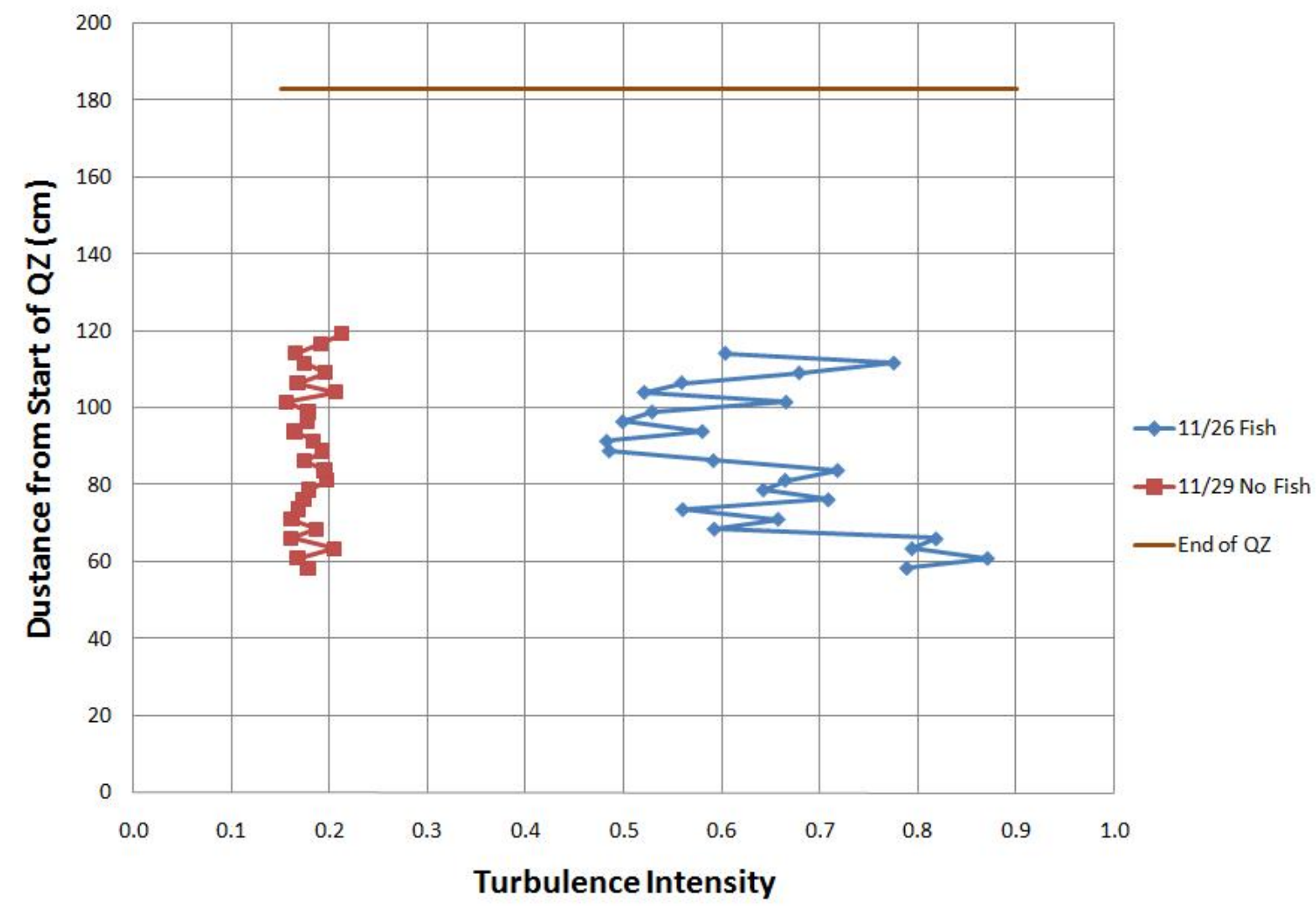

Figure 52: Streamwise Profiles of Turbulence Intensities

Figure 52 represents the streamwise profile of turbulence intensities corresponding to Figures 32-33.

The measured profiles in Figure 52 vary in shape and magnitude. The measured turbulence intensity profile measured on November 29 is more constant when traversing in the streamwise direction than the November 26 profile. From the previous observation, it may be stated that a smaller screen is allowing for a more constant streamwise turbulence intensity profile. A conclusion is drawn that a larger flow rate will increase the magnitude of turbulence in the quiescent zone.

\subsubsection{Vertical Profiles of Reynolds Stresses}

The following figures represent the Reynolds stress profiles corresponding to Figures 2629 (calculated using Equations 18-23). As mentioned in Section 4.1.2, the Reynolds stresses are 
caused by correlated fluctuations in the turbulent flow. The fluctuations are averaged over a 360 second time interval.

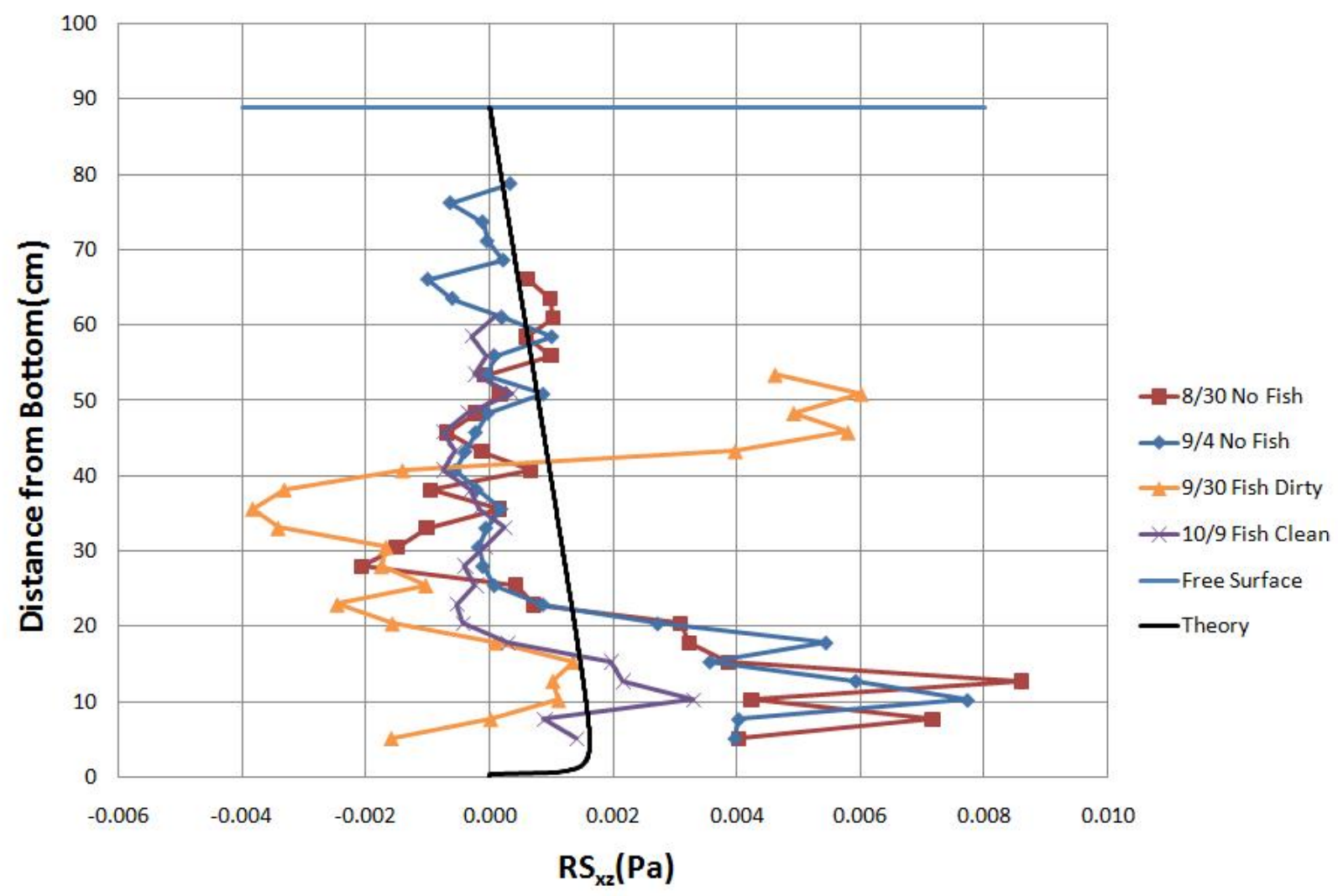

Figure 53: Vertical Profiles of $\mathbf{R S}_{\mathrm{xz}}$

Figure 53 shows the vertical profile of $\mathrm{RS}_{\mathrm{xz}}$ (discussed in Section 4.1.2) corresponding to measurements taken in Figures 26-29.

The measured profiles in Figure 53 (excluding September 30) all possess a similar shape, but vary in magnitude. The shape of the previously mentioned profiles are similar to the theoretical profile (calculated using Equations 26 and 29), each having the largest magnitude of $\mathrm{RS}_{\mathrm{xz}}$ in the 10 to $15 \mathrm{~cm}$ range from the bottom of the quiescent zone, then decreasing towards zero as the free surface is approached. This maximum magnitude corresponds to the same location as the maximum magnitude in the vertical $w_{R M S}$ profile (Figure 49). As seen in the vertical turbulence intensity profiles, the measured profile of $\mathrm{RS}_{\mathrm{xz}}$ on October 9 has the smallest magnitude compared to the other measured profiles (excluding September 30). This also supports the idea that the small screen decreases the size of the larger eddies and reduces $\mathrm{RS}_{\mathrm{xz}}$. The measured profile on September 30 suggests that a dirty screen causes the $\mathrm{RS}_{\mathrm{xz}}$ profiles to 
vary erratically. The effects of angular sensitivity and misalignment may be a factor in the differences of measured profiles; however the dirty screen consistently causes drastic differences when compared to other measured profiles, concluding that the screen is a realistic factor.

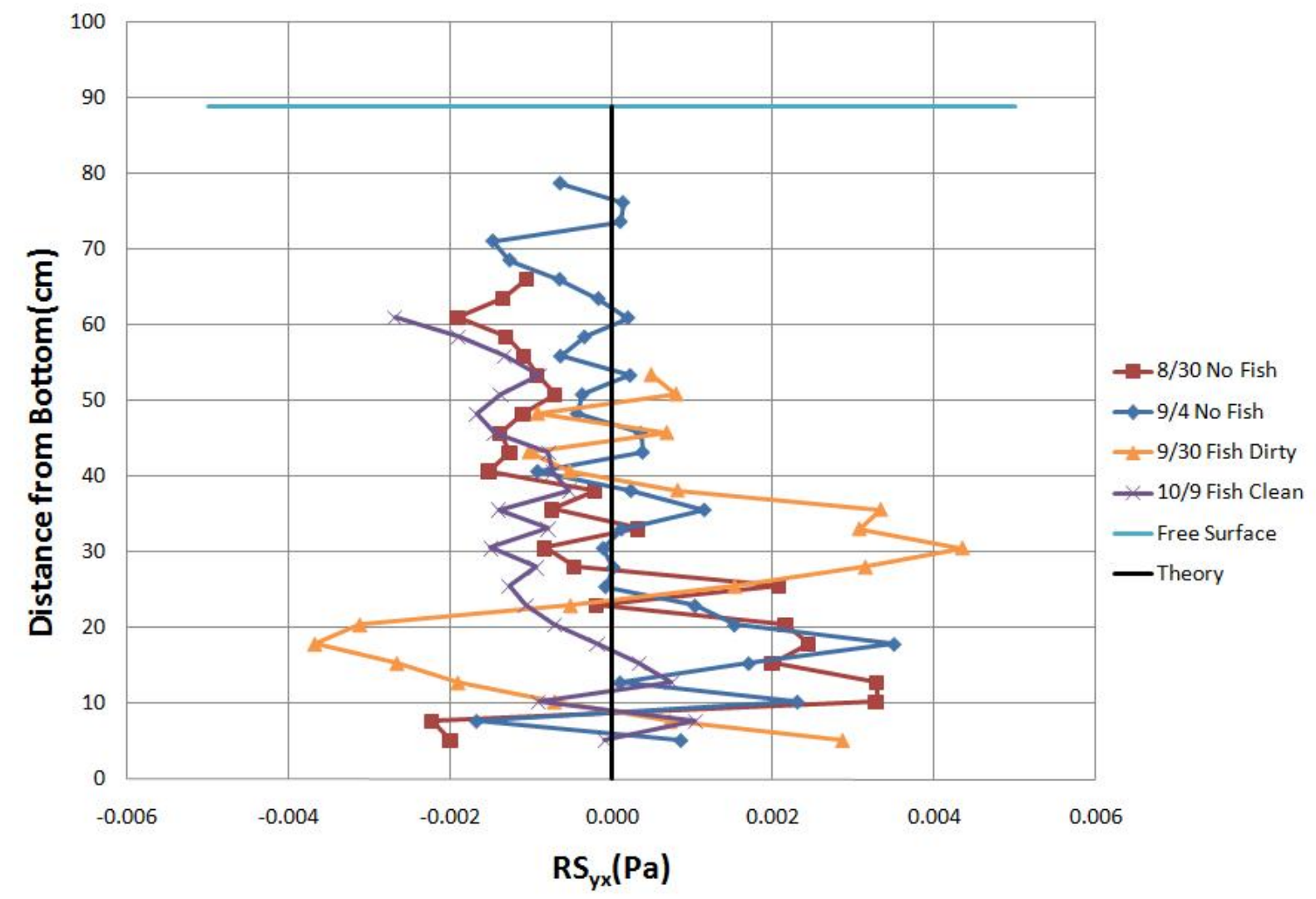

Figure 54: Vertical Profile of $\mathbf{R S}_{\mathbf{y x}}$

Figure 54 shows the vertical profile of $\mathrm{RS}_{\mathrm{yx}}$ (discussed in Section 4.1.2) corresponding to measurements taken in Figures 26-29.

Since all the profiles in Figure 54 are measured at the center of the quiescent zone, the theoretical result for the Reynolds stress vertical profile is a constant value of zero from the bottom of the quiescent zone to the free surface. Instantaneously, there may be changes in momentum at the center of the quiescent zone. However, since the fluctuations are averaged over 360 seconds, those in the transverse direction are contributing to changes in $x$-momentum on the vertical center line and will average to zero due to the symmetry of the theoretical transverse profile of $\bar{u}$ (Figure 40). Statistically, the average of the positive and negative $x$ momentum changes (due to fluctuations) is zero at the center of the quiescent zone. Since the measured transverse profile of $\bar{u}$ (Figure 40) is not symmetric with respect to the center line, the 
positive and negative momentum changes (due to fluctuations) do not cancel each other out. When observing the previously mentioned profiles, the profile measured on October 9 has the smallest maximum magnitude. This supports the concept that the small screen decreases the magnitude of the fluctuations, resulting in a decrease of $\mathrm{RS}_{\mathrm{yx}}$.

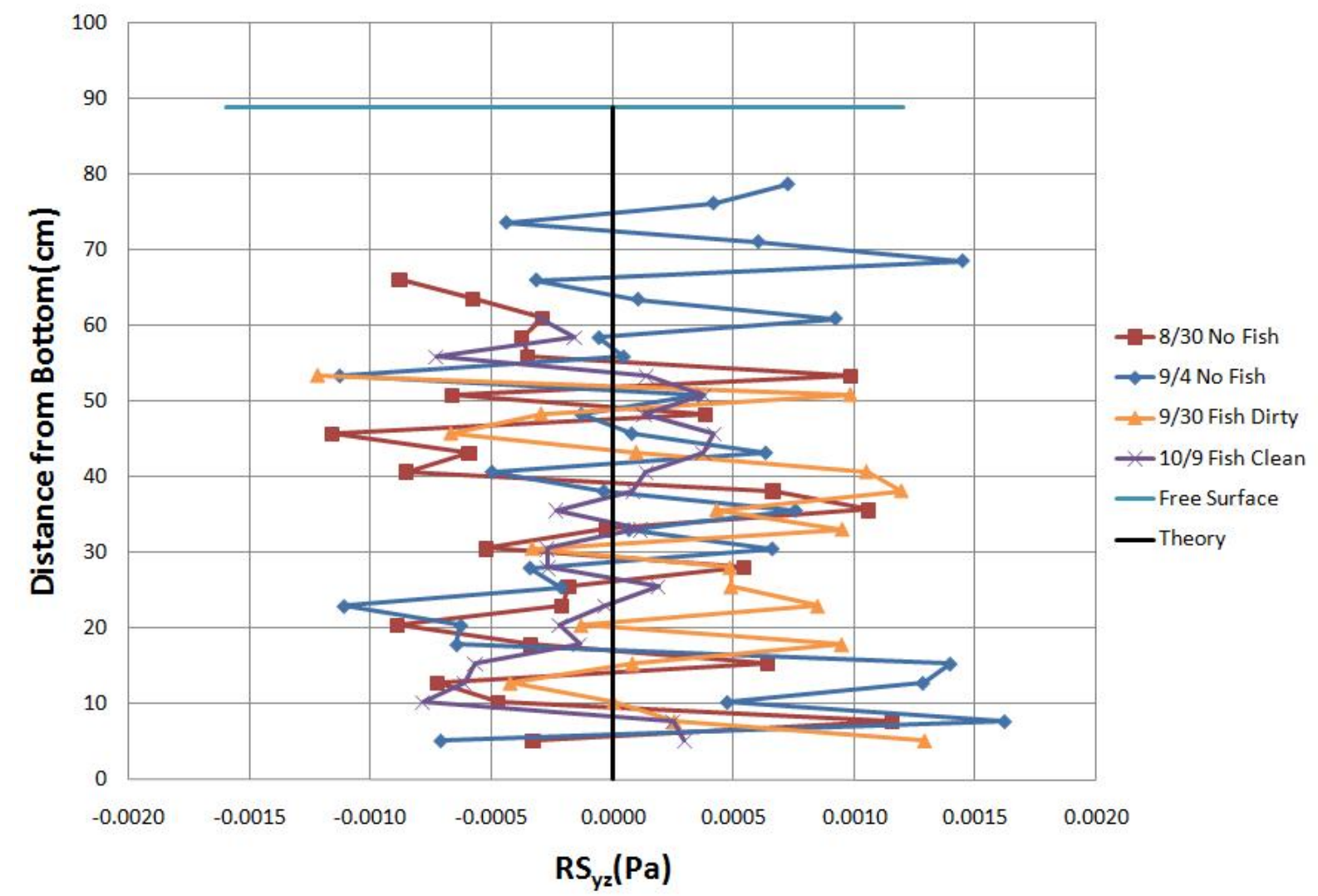

Figure 55: Vertical Profiles of $\mathbf{R S}_{\mathrm{yz}}$

Figure 55 represents the vertical profile of $\mathrm{RS}_{\mathrm{yz}}$ (discussed in Section 4.1.2) corresponding to measurements taken in Figures 26-29.

For all measured profiles in Figure 55, the Reynolds stresses appear to fluctuate on either side of the theoretical zero line. This is expected since the measured transverse and vertical mean velocities are non-zero and do not possess symmetry with respect to either vertical or transverse center lines. The magnitudes of the $\mathrm{RS}_{\mathrm{yz}}$ measured on October 9 are the smallest in Figure 55. The previous observation also supports the concept of the small screen reducing magnitudes of fluctuations, resulting in smaller magnitudes of $\mathrm{RS}_{\mathrm{yz}}$. 


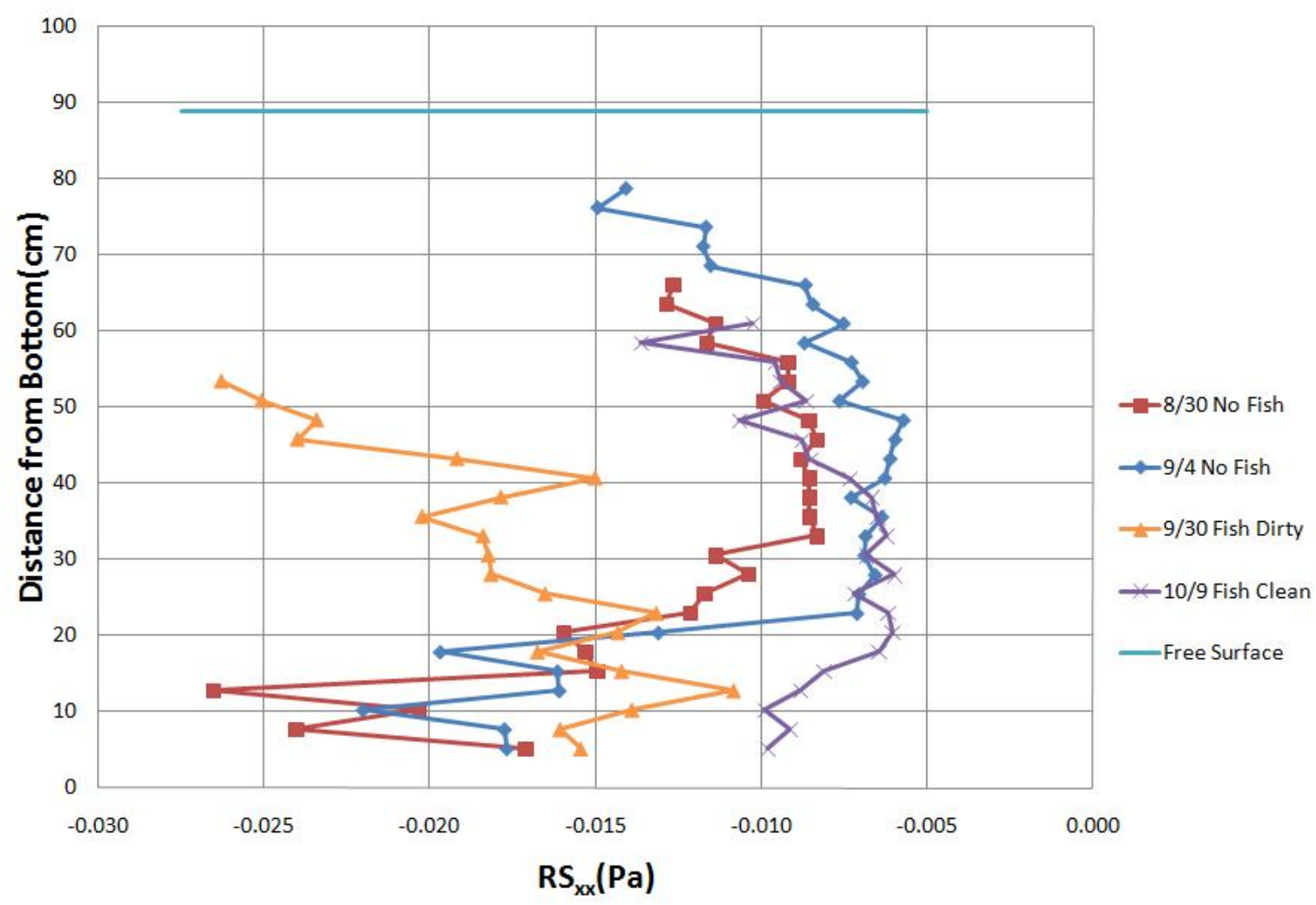

Figure 56: Vertical Profiles of $\mathrm{RS}_{\mathrm{xx}}$

Figure 56 represents the vertical profile of $\mathrm{RS}_{\mathrm{xx}}$ (discussed in Section 4.1.2) corresponding to measurements taken in Figures 26-29. There is no theoretical approximation for $\mathrm{RS}_{\mathrm{xx}}$.

When observing the measured profiles in Figure 56, it can be observed that the profile measured on October 9 has the smallest average value of $\mathrm{RS}_{\mathrm{xx}}$. 


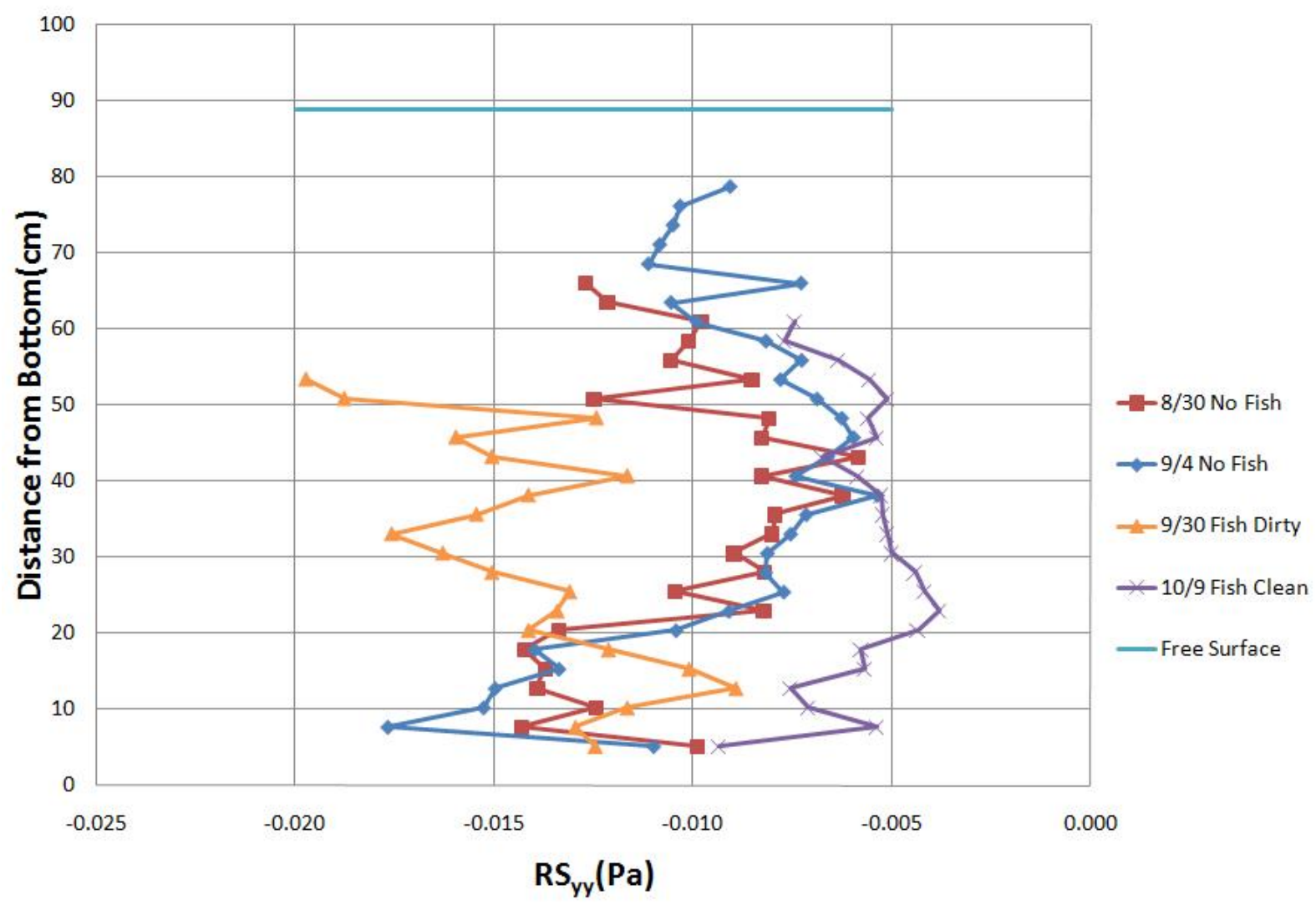

Figure 57: Vertical Profiles of $\mathbf{R S}_{\mathbf{y y}}$

Figure 57 represents the vertical profile of $\mathrm{RS}_{\mathrm{yy}}$ (discussed in Section 4.1.2) corresponding to measurements taken in Figures 26-29. There is no theoretical approximation for $\mathrm{RS}_{\mathrm{yy}}$.

Again, the profile measured on October 9 has the smallest magnitude of Reynolds stress. The major cause of increase in fluctuations may be due to debris blocking the screen in certain regions. When analyzing Figure 57, it can be observed that a major difference in flow rate will cause a drastic difference in the magnitude of turbulence. This may support the idea that debris blocking areas of the screen will cause the flow to travel through less obstructed areas. This would increase flow velocities in unobstructed regions and cause the magnitude of turbulence to increase in these regions. Conversely, it may be concluded that a low magnitude of turbulence is present in heavily obstructed regions of the screen. 


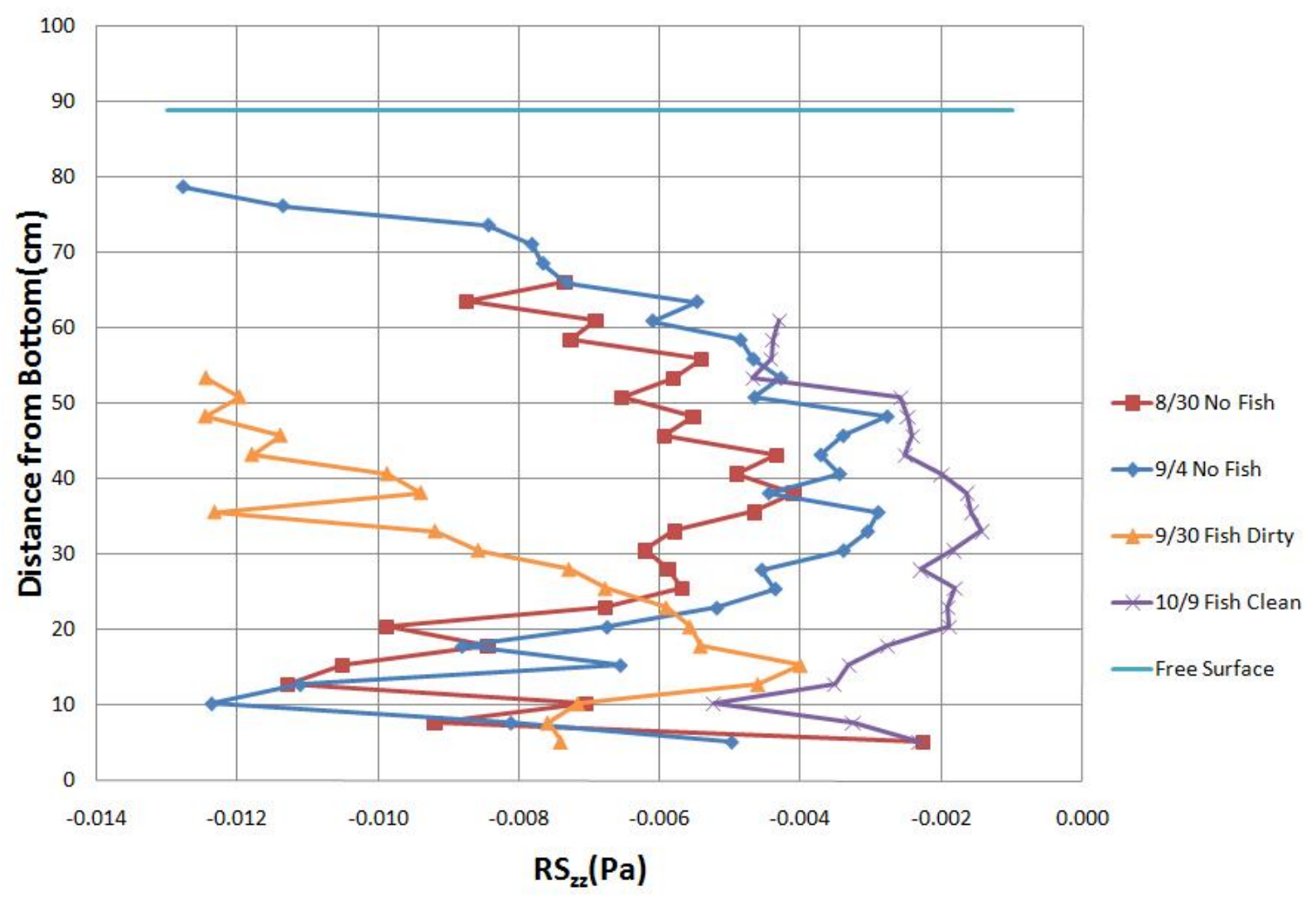

Figure 58: Vertical Profiles of $\mathbf{R S}_{\mathrm{zz}}$

Figure 58 represents the vertical profile of $\mathrm{RS}_{\mathrm{zz}}$ (discussed in Section 4.1.2) corresponding to measurements taken in Figures 26-29. There is no theoretical approximation for $\mathrm{RS}_{\mathrm{zz}}$.

Again, the profile measured on October 9 has the smallest magnitudes of Reynolds stresses in Figure 58.

\subsubsection{Energy Spectrum}

The following log-log plots represent the energy spectrum corresponding to the frequency of fluctuations in velocities measured with SonTek's 16 Mhz Micro ADV. Energy spectra at specified points are created at three different distances from the bottom using measurements taken on September 4. Figures 59-62 show the energy spectra of the streamwise, transverse, and vertical velocity fluctuations.

According to Kolmogorov, three different ranges corresponding to each measurement location should be present. The straight line represents the log-log plot of the frequency raised to 
the $-5 / 3$ power. A slope of $-5 / 3$ is used to determine the inertial sub-range; where energy from large eddies is transferred to smaller eddies.

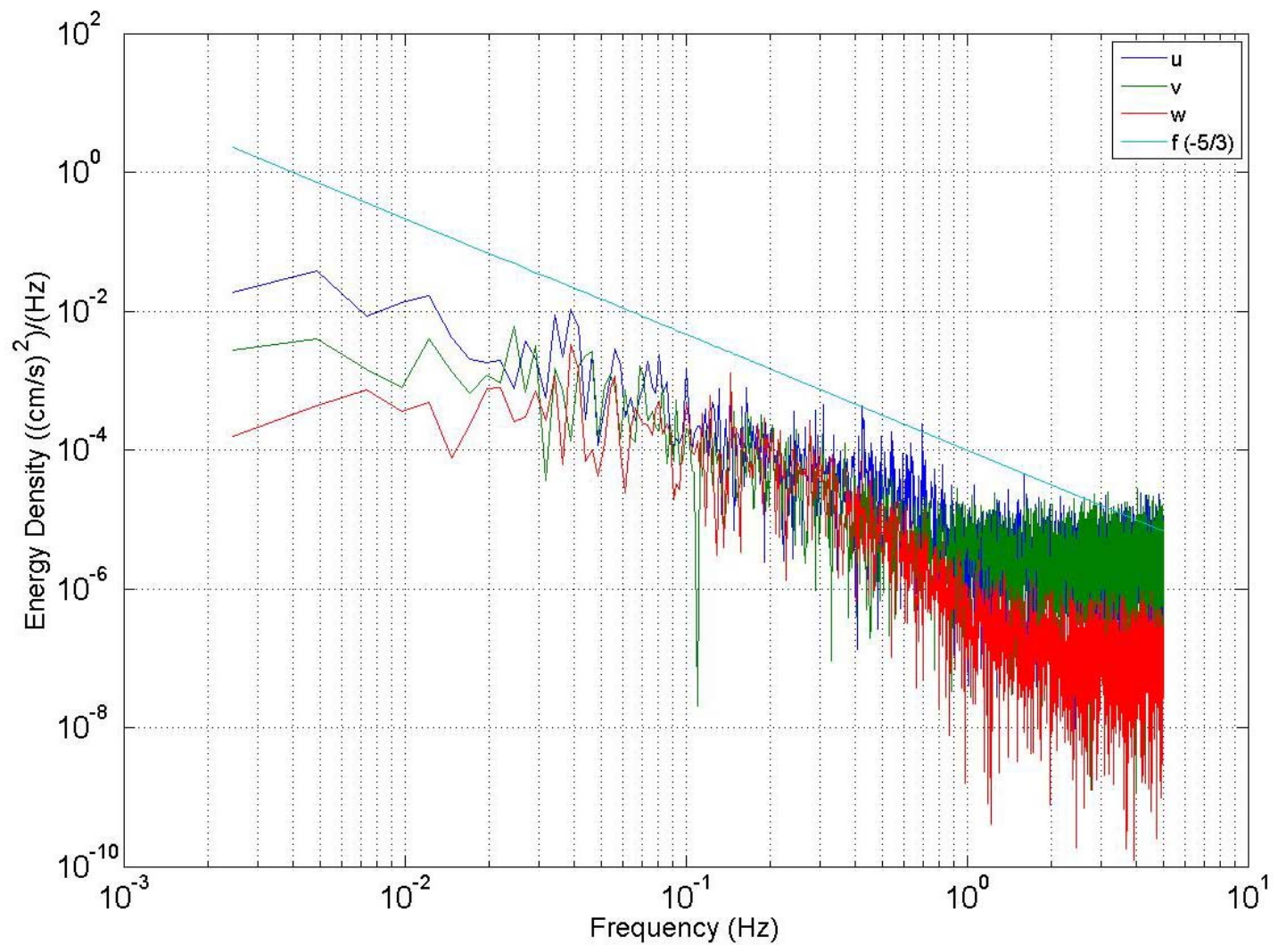

Figure 59: Energy Spectrum at $10 \mathrm{~cm}$ from Bottom of Quiescent Zone from Measurements on September 4, 2008 


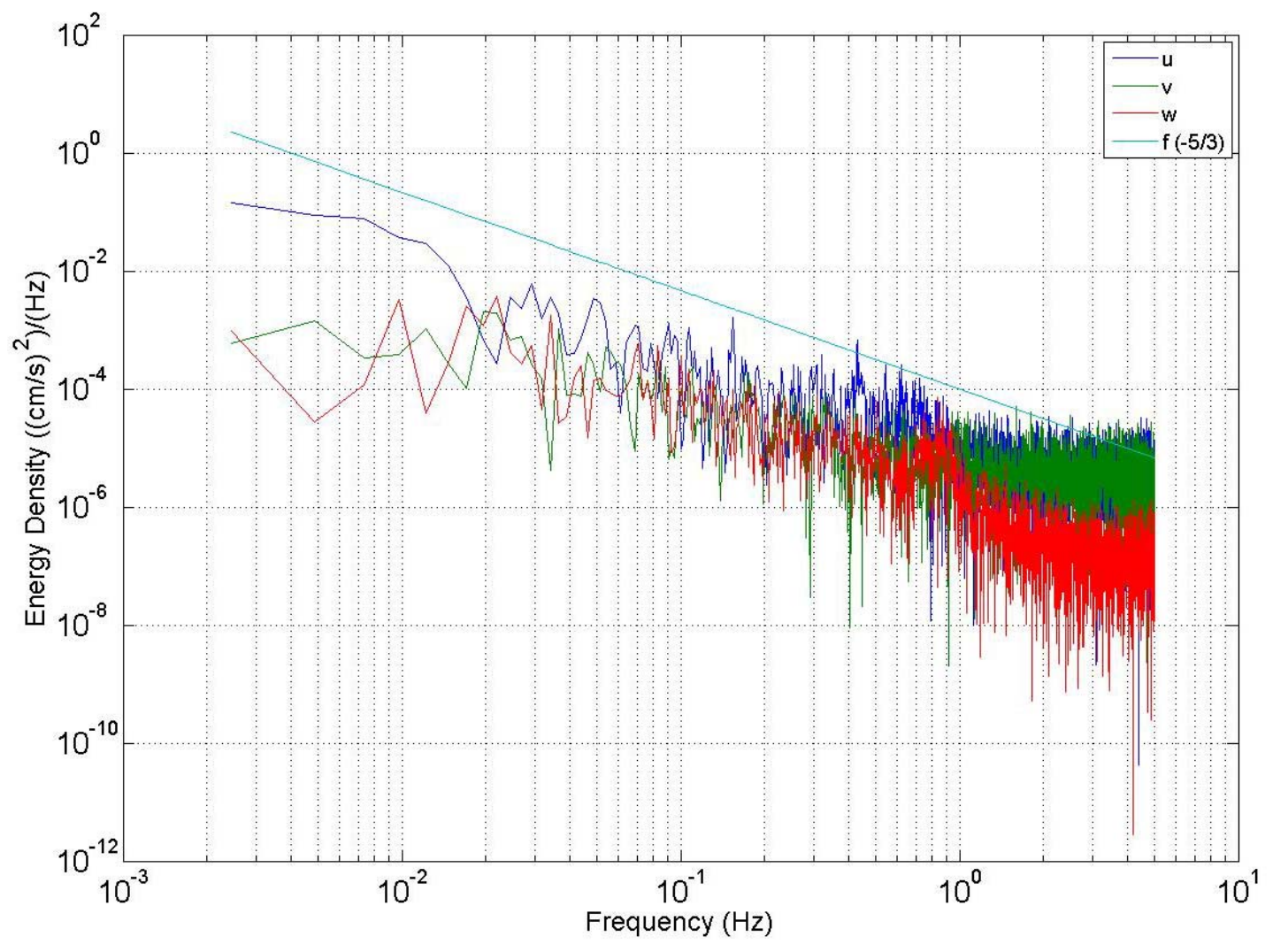

Figure 60: Energy Spectrum at $43 \mathrm{~cm}$ from Bottom of Quiescent Zone from Measurements on September 4, 2008 


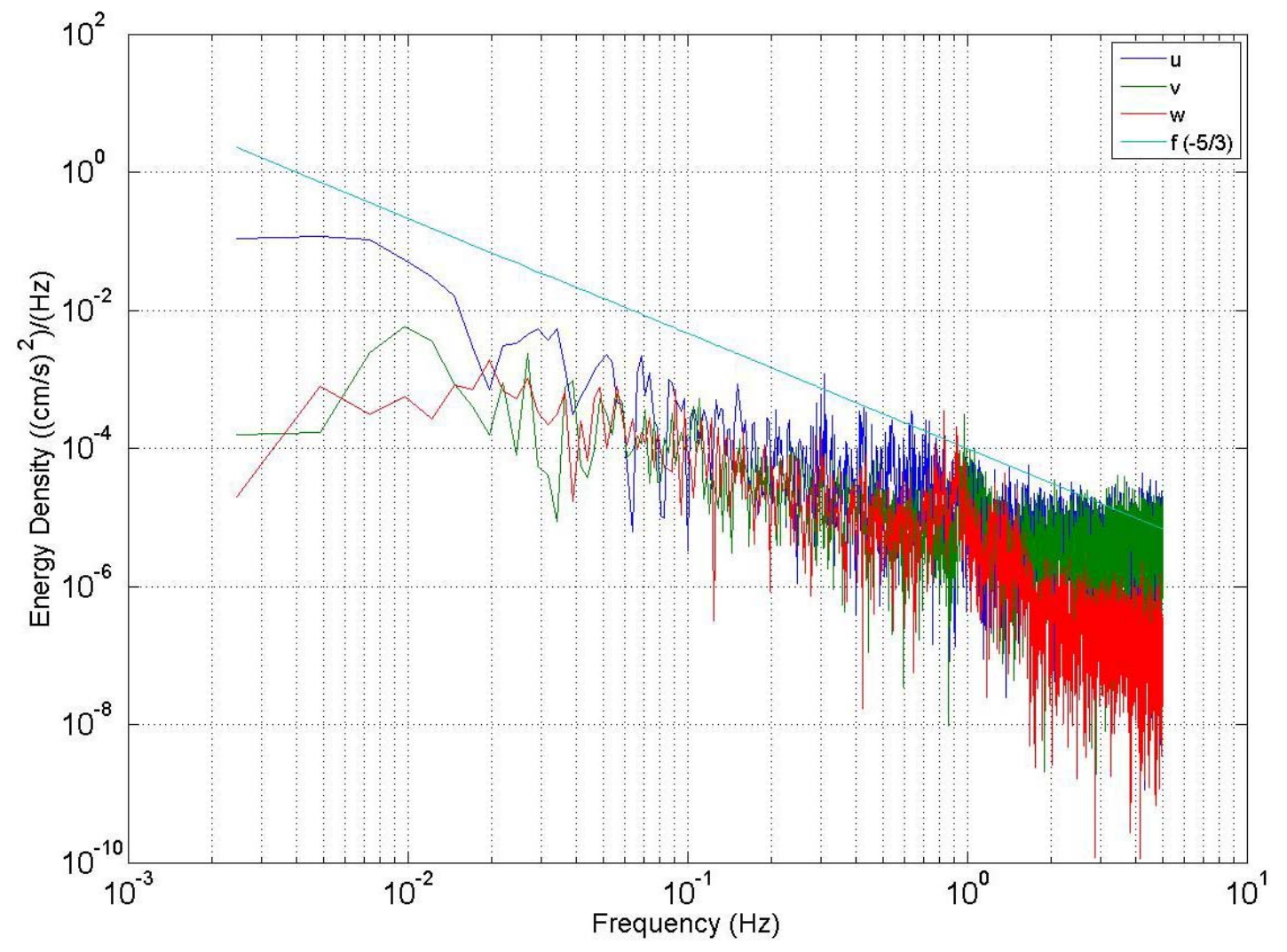

Figure 61: Energy Spectrum at $61 \mathrm{~cm}$ from Bottom of Quiescent Zone from Measurements on September 4, 2008 


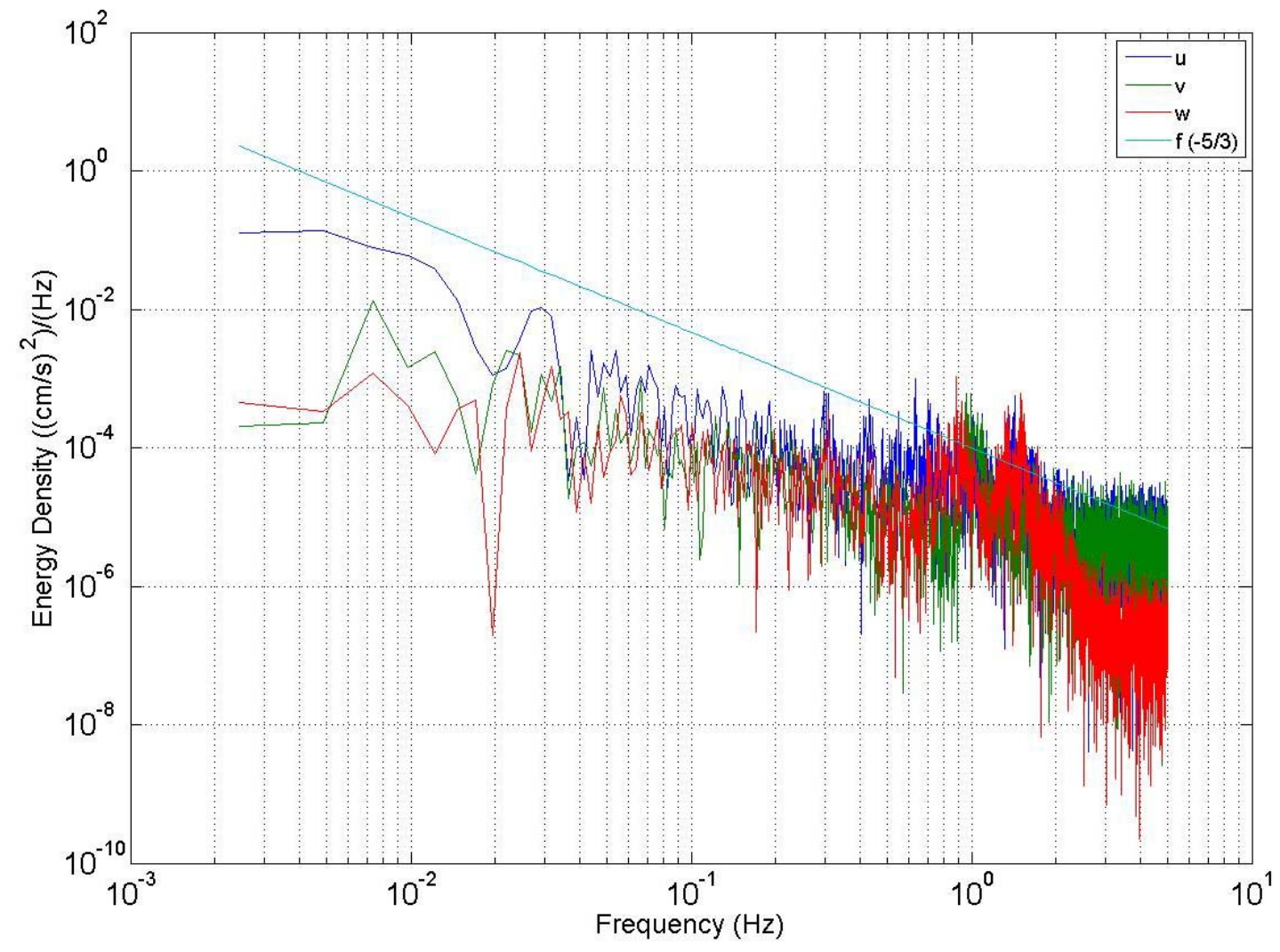

\section{Figure 62: Energy Spectrum at $79 \mathrm{~cm}$ from Bottom of Quiescent Zone from Measurements on September 4, 2008}

When looking at Figures 59-62, it is difficult to segregate the large eddy range, inertial sub-range, and the energy dissipation range. The inertial sub-range of the signals can be determined by finding (-5/3) slope in Figures 59-62. It is difficult to determine the inertial subrange for all components of velocities in Figures 59-62. This may be result of the relatively low Reynolds number associated with the flow (Trowbridge et al., 1989). If the flow had a larger ratio of large scale inertial eddies to small scale viscous eddies, the inertial sub-range may be easier to determine. In turn, this may allow the large eddy range and dissipation range to stand out.

The energy spectra in Figures 59-62 are not smooth. It appears that the spectra become rougher as the distance from the bottom increases. This may be due to random fluctuations caused by free surface disturbances (such as wind shear) and unsteady flow. Differences in flow rate were observed on different days, although the position of the inlet valve from the reservoir did not change. The causes of change in flow rate were undetermined, but may have been 
caused by disturbances or water level changes in the reservoir (Dogwood Lake) on particular days.

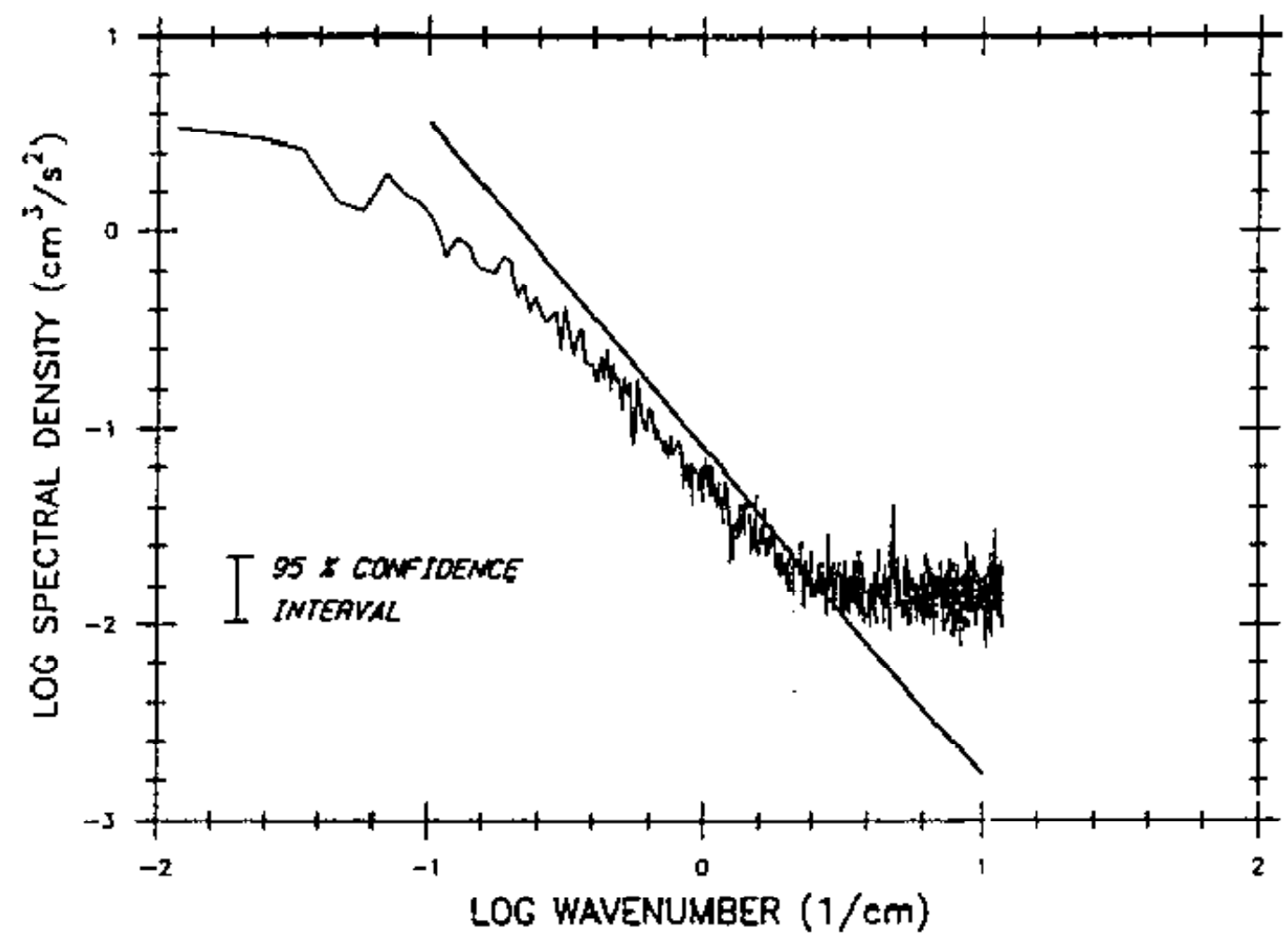

Figure 63: Energy Spectrum Measured in a Flume (Throwbidge et al., 1989)

At high frequencies it appears that the energy is relatively constant. This phenomenon is common and can also be seen in measurements taken by Trowbridge et al. (1989) in a $17 \mathrm{~m}$ long laboratory flume (Figure 63). The constant energy section relating to high frequencies is most likely a noise floor. The source of the noise floor is most commonly Doppler noise (frequency shifts measured by the ADV from sound waves reflecting off objects that are not located in the sampling volume) and mechanical vibrations from the probe and/or traversing mechanism. Lohrmann et al. (1995) states that Doppler noise is most commonly found in the flat section of the energy spectrum approaching the Nyquist frequency (sampling frequency/2). This allows the flat section of the energy spectrum to be ignored. The Doppler noise associated with transverse velocities is approximately four times the magnitude of the noise associated with the vertical velocities due to the geometry of the probe (SonTek, 2001). 


\subsection{Material in Appendices}

\subsubsection{Appendix A: Tables of Measured Values}

Appendix B includes values of flow rates, water temperatures, mean velocities, RMS velocity, Reynolds stress, turbulence intensity, mean correlation (average correlation between all velocity components), mean SNR, and standard deviation.

\subsubsection{Appendix B: Mean Velocity Profiles with Standard Deviation}

Appendix $\mathrm{C}$ contains all mean velocity profiles measured with error bars representing the standard deviation.

\subsubsection{Appendix C: Additional Profiles}

This appendix contains additional RMS velocity profiles, and Reynolds stress profiles measured on the same days as the profiles in the body of this paper.

\subsection{Summary and Conclusion}

Several velocity profiles were measured in different quiescent zones at Dogwood Lake's rectangular aquaculture raceway system using SonTek's Micro ADV. The measurements were collected following SonTek's (2001) guidelines, allowing accurate velocity measurements to be obtained. The velocity time series were used to calculate traditional measures of turbulent flow such as Reynolds stress profiles, turbulence intensity profiles, and RMS profiles. Using SonTek's Micro ADV yielded improvements of previous research pertaining to velocity measurements taken in the quiescent zone of rectangular aquaculture raceway systems. Improvements that were made include: a more detailed vertical profile of mean streamwise velocity, using adequate averaging times for measuring mean velocities, and sampling at faster rates to capture smaller scale turbulent fluctuations in the velocities. The improved velocity profiles and turbulence characteristic profiles were then compared to two-dimensional, fully developed, turbulent open channel flow over smooth surfaces. It is concluded that the flow through the quiescent zones at Dogwood Lake does not contain characteristics of twodimensional, fully developed, turbulent open channel flow over smooth surfaces. Several factors were found that may have caused this deviation between the theoretical and measured flow characteristics at Dogwood Lake. 


\subsubsection{Disturbances in the Raceway System at Dogwood Lake}

Influential factors causing discrepancies between the theoretical and measured characteristics in the flow are disturbances caused by the presence of fish, screens, and wind shear on the free surface.

\subsubsection{Fish and Screen Disturbances}

It was difficult to determine if the upstream presence of fish or the difference in the upstream screens were causing discrepancies between measured mean velocity profiles. In most of the measured mean velocity profiles, obvious discrepancies were found between the measurements in the quiescent zone containing fish upstream and measurements in the quiescent zone without fish upstream. The absence of fish and a large screen upstream of the measurements produced mean velocity profiles with characteristics similar to two-dimensional, fully developed, open channel flow characteristics in some regions. The presence of fish and a small screen upstream created larger discrepancies between measured and theoretical characteristics, and more scatter in mean profiles compared to measurements taken with zero fish and a large screen upstream. Since the quiescent zone with fish upstream contains a small screen and the quiescent zone without fish upstream contains a large screen, it is difficult to determine which factor is the greater cause of the discrepancies. If it is assumed that the fish will always increase the turbulence in the quiescent zone, then it may also be assumed that the screen alone is causing the reduction in turbulence that is most obviously seen in Figure 50. The small screen appeared to limit the magnitude of turbulence intensities, Reynolds stresses, and RMS for all components of velocity. It was found that the level of turbulence is not reduced when the small screen is obstructed by debris. A screen containing blockage resulted in larger magnitudes of turbulence intensities, Reynolds stresses, and RMS when compared to a clean screen. An obstructed screen may also be diverting the flow away from the blocked sections, therefore increasing the flow in unobstructed sections. This creates more energy for larger fluctuations in the velocity to occur. Also, debris in the screen is likely diverting the flow in transverse and vertical directions causing directional components of mean transverse and vertical velocities. Profiles resulting in non-zero mean transverse velocities were validated by a dye test conducted in quiescent zones A4 and B4, proving that transverse velocities exist in the quiescent zones at Dogwood Lake. 


\subsubsection{Free Surface Disturbances}

The magnitude of RMS and turbulence intensities increased as the distance to the free surface decreased, probably due to waves and wind induced currents.

\subsubsection{Unsteady Flow}

Mean steady flow is an important assumption used in the derivations of the theoretical equations. The presence of a time varying (unsteady) flow can affect all flow characteristics. A 360 second averaging time was found to yield a stable average on a particular day; however, if the flow rate was not varying within that particular time interval, the effects of unsteady flow may not have been noticed. The flow at Dogwood Lake appeared to vary with respect to time. The time variation of the flow rate was noticed via discrepancies between flow rates measured on different days. The time rate of change in the flow rate may have been due to the swaying motions of level variations in the reservoir.

\subsubsection{Low Reynolds Number}

A low Reynolds number $\left(2 \times 10^{4}\right)$ shows that the flow is mildly turbulent. This turbulence is present in all measurements. The large eddy range, inertial sub-range, and dissipation range were difficult to segregate. This may be a result of the relatively low Reynolds number associated with the flow (Trowbridge et al., 1989). If the flow had a larger ratio of large scale inertial eddies to small scale viscous eddies (i.e., larger Reynolds number), the inertial subrange may be easier to determine and may allow the large eddy range and dissipation range to be more distinguishable.

\subsubsection{Human Error}

Angular sensitivity (Section 6.1.1) is a possible cause of discrepancies between measured and theoretical flow characteristics. Measurements were taken carefully so that the ADV probe is consistently aligned in the same orientation for each measurement point. A misalignment with respect to other measurement locations will be small and unnoticeable to the eye. It has been estimated that misalignment of less than $\pm 1^{\circ}$ about the vertical axis is present for all measured profiles. A misalignment of $\pm 1^{\circ}$ will cause error in the mean streamwise velocities, mean transverse velocities, and mean vertical velocities of $-0.080 \mathrm{~cm} / \mathrm{s},+0.59 \mathrm{~cm} / \mathrm{s}$, and $-0.21 \mathrm{~cm} / \mathrm{s}$ respectively. This allows for the conclusion that angular sensitivity has a significant effect on 
measured mean transverse velocities and measured mean vertical velocities, but has negligible effects on the measured mean streamwise velocities.

The traversing mechanism creates uncertainty in the velocities when comparing measurements taken on different days. Marks are placed on the traversing mechanism to allow all components to be placed in the same location. Uncertainty of placement of these components on different days is estimated at $\pm 2 \mathrm{~cm}$ for all directions with a $95 \%$ confidence interval. The results show (Tables 4-6) that mean transverse and vertical velocities as well as Reynolds stresses will be affected by this shift. This may be a cause of variation in mean transverse profiles, mean vertical velocity profiles, and Reynolds stress profiles measured on different days. The repetition of drastic variations in profiles measured with a dirty screen compared to a clean screen supports the conclusion that variations in profiles actually exist and are not purely a result of angular sensitivity and misalignment. Small meshed screens are used in wind tunnels in order to reduce the magnitude of turbulence. Likewise, a small meshed screen reduces the magnitude of turbulence intensity in the quiescent zones. This is another factor proving that the results are not purely due to traversing mechanism misalignment and angular sensitivity.

\subsubsection{Uncertainty of the ADV}

Uncertainty exists in all experiments. As discussed in Section 5.1.2, errors resulting from the uncertainty of the ADV may be ignored. Averaging over a 360 second time interval at a relatively slower sampling rate $(10 \mathrm{~Hz})$ allowed the error resulting from this noise to be reduced to $\pm 1.4 \times 10^{-3} \mathrm{~cm} / \mathrm{s}$ for mean streamwise and transverse velocities, and $\pm 3.4 \times 10^{-4} \mathrm{~cm} / \mathrm{s}$ for mean vertical velocities. This error is small and ignored for all mean measurements.

\subsubsection{Recommendations}

It is recommended that a flow meter be added to the inlet pipe that carries water from the reservoir (Dogwood Lake) to the raceway system. This will allow quick and easy checks of the flow rate. An orifice meter is suggested since it is relatively inexpensive and easy to install. 


\section{Part 2: Solid Removal System}

\subsection{Introduction}

Removing solids from an aquaculture system is known to be a labor intensive job. Solids (uneaten food and fish waste) in the rectangular aquaculture raceway system at Dogwood Lake settle in an area called the quiescent zone located at the end of each raceway. These solids must be removed in order for the fish to have a healthy living environment. Currently, the quiescent zones are cleaned manually via a standpipe system. Cleaning is preformed approximately 2-5 times a week. The cleaner removes the standpipe (Figure 64) and sweeps the settled solids into the standpipe hole. A majority of the particles are removed by the standpipe hole; however, some are suspended from the bottom and flow over the weir to the next raceway. The solids that enter the standpipe hole flow through piping that leads down the hill (Figure 65) to the filtering system. The current filtering system at Dogwood Lake is called the geo-tube. The geo-tube is a large permeable bag that allows water to pass through and solids to be collected.

The West Virginia University (WVU) Agriculture \& Natural Resources department requested the design of a cleaning mechanism to waste little water, require little maintenance, and be installed and removed easily. The system is to be installed while the raceway is fully operational. Presently, there is no available electricity supply at Dogwood Lake. 


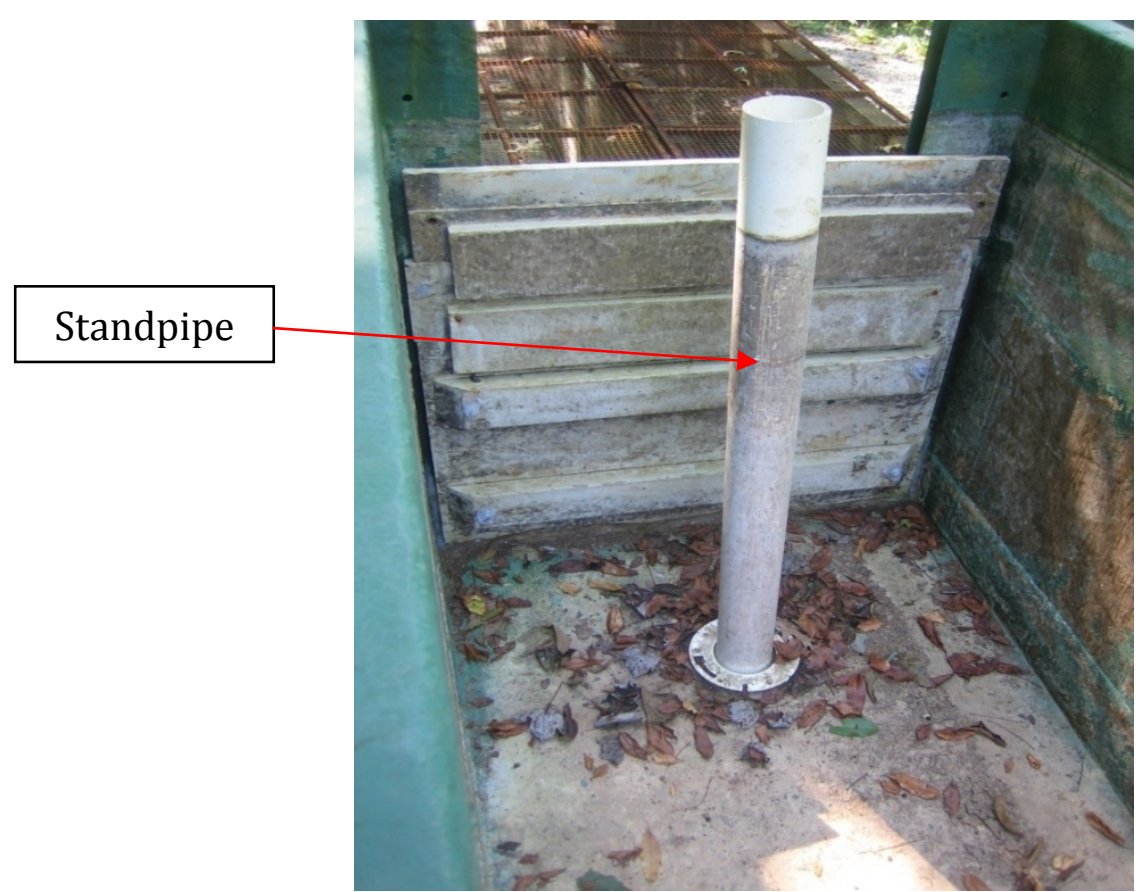

Figure 64: Standpipe in Dry Quiescent Zone at Dogwood Lake

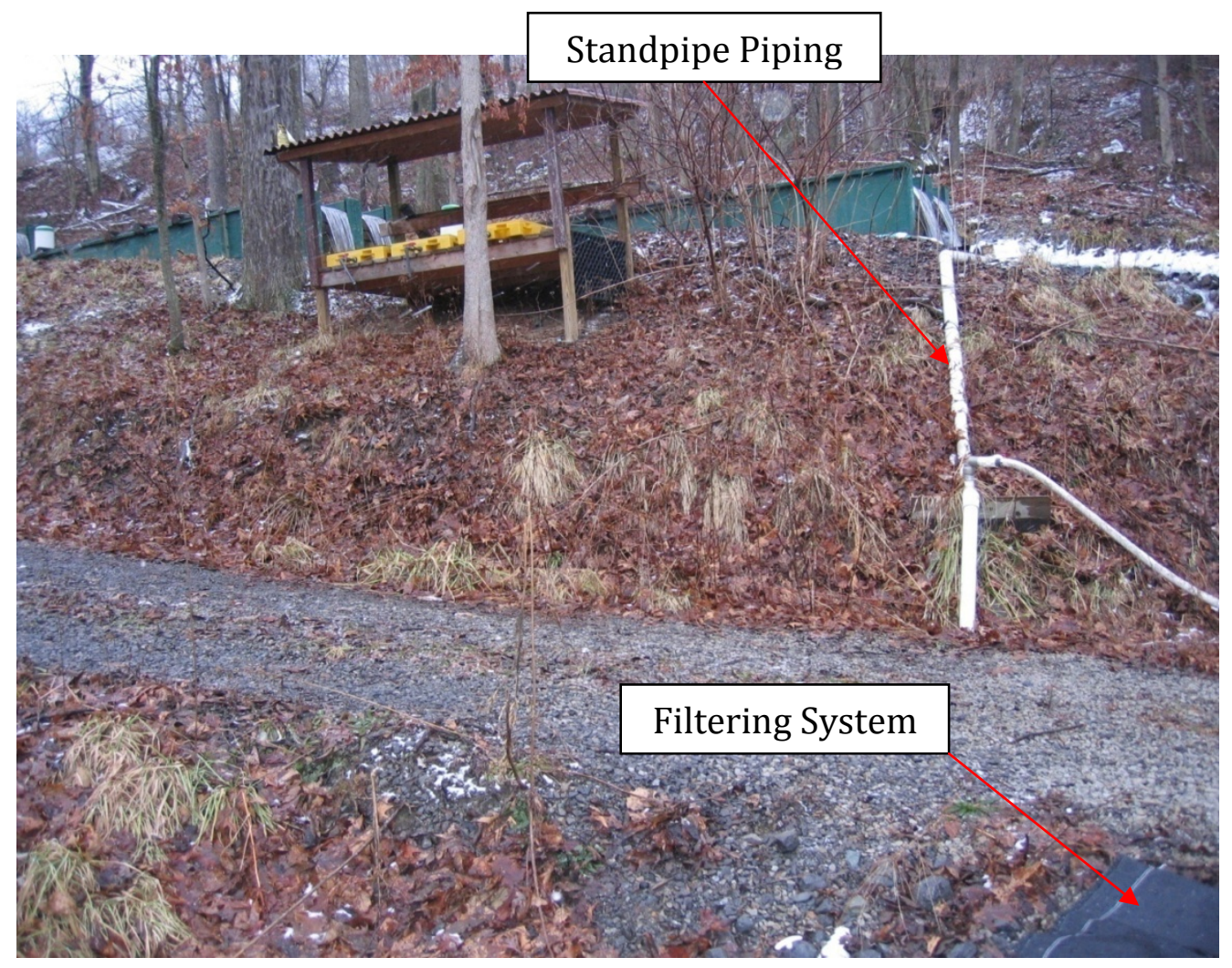

Figure 65: Piping from the Current Standpipe System at Dogwood Lake 


\subsection{Objective}

The objective was to design an efficient, low maintenance system for removing settled solid particles from the entire bottom of the quiescent zone. The design should not continuously remove water from the quiescent zone other than the overflow from the weir, and should be installed and removed without disrupting the structure of the raceway system (i.e. drilling holes into the walls). The cleaning system should limit the suspension of particles from the bottom of the quiescent zone and be cost effective. The design of the system is to be specific to the raceway system at Dogwood Lake.

\subsection{Design Procedure}

The first step is to consider several possibilities for design, while considering all constraints. The following subsections briefly describe the possible ideas for the design of the cleaning system.

\subsubsection{Possible Design Ideas}

\subsubsection{Siphon System (SS)}

A three-dimensional drawing of the siphon system can be seen in Figure 66. A small portion of water flowing over the weir enters the fill pipe. Water from the fill pipe fills the siphon tub and a buoyant object opens a flap valve when the water level in the siphon tub reaches a critical height. This allows water to rush into the siphon pipe and begin removing air. When the water level in the siphon tub drops, the flap valve closes and the remaining air is removed, allowing the siphon to activate. When the water level in the quiescent zone drops to a critical height, holes drilled in the pipe connected to the false bottom (FB-pipe) become exposed to air, and air will enter the system. When air enters back into the system, the siphon is deactivated and the cycle repeats (a more detailed description is discussed in Section 13.7.0).

Solids and water in the quiescent zone are removed via holes in the top surface of the false bottom (Figure 67) and filtered out by a filtering system when the siphon is active. The current filtering system at Dogwood Lake could be used as the filtering system for the siphon system. The false bottom consists of a rectangular volume with holes drilled in the top surface to create an even profile of suction on the bottom of the quiescent zone. 


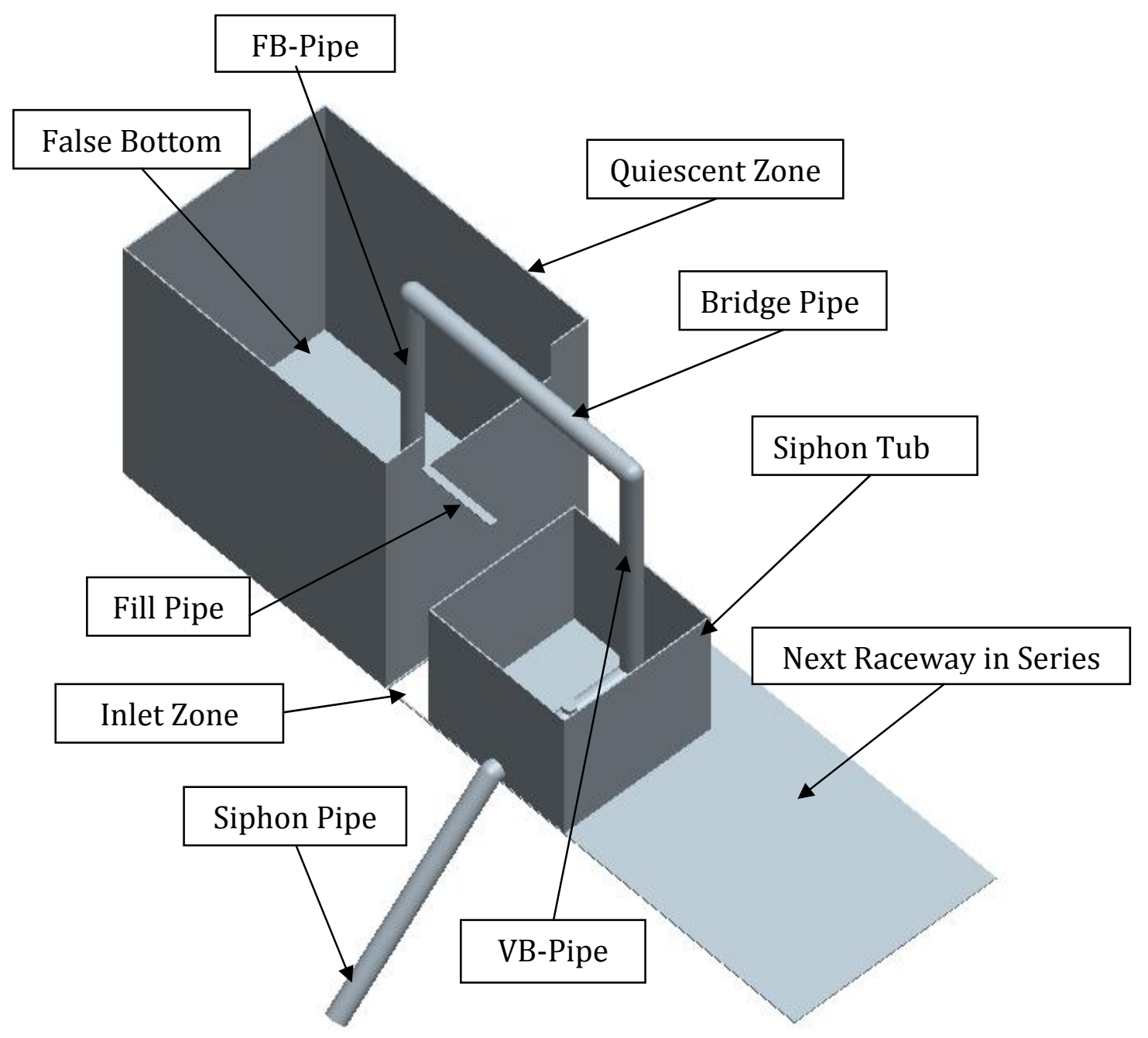

Figure 66: Siphon System 

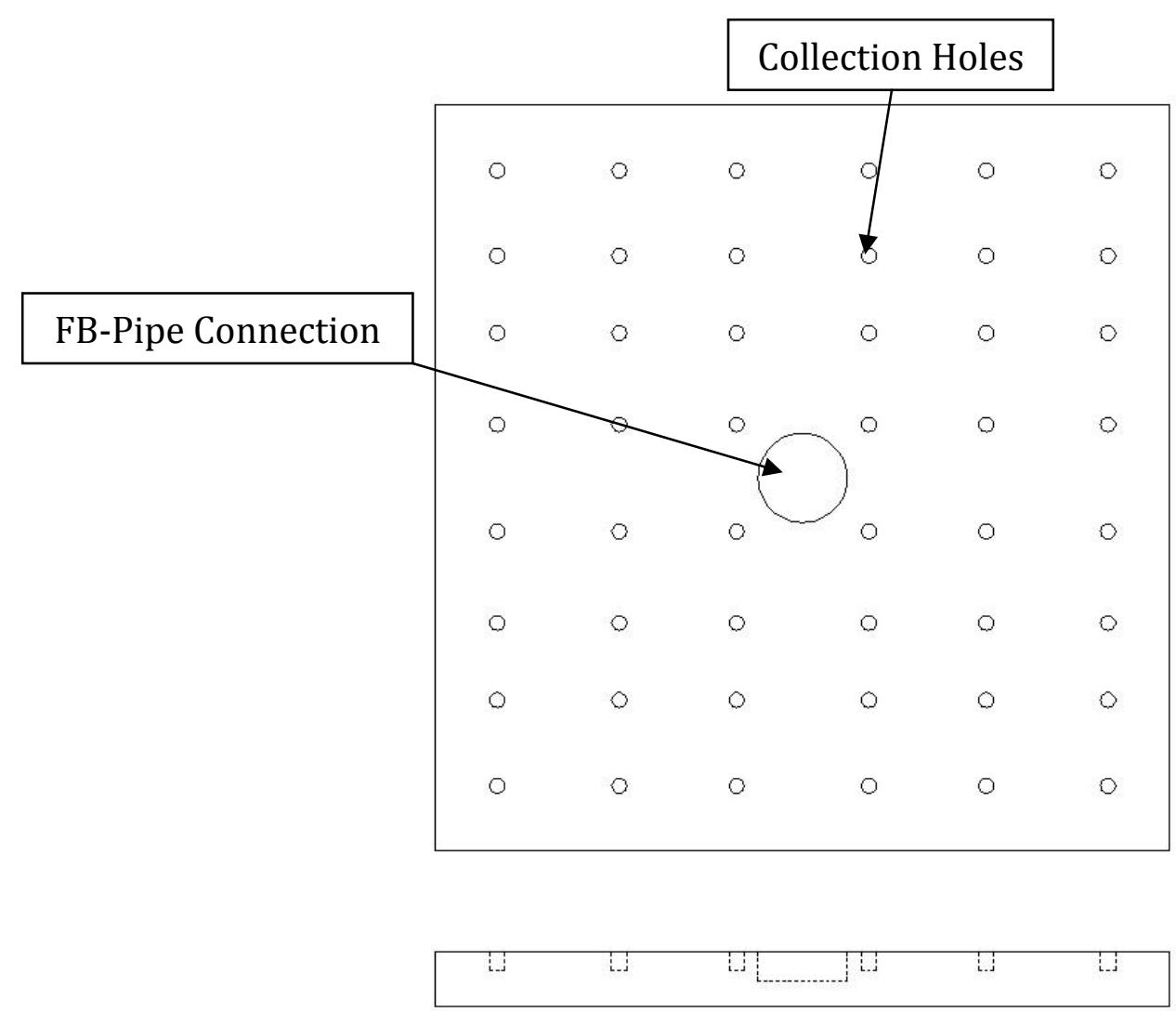

Figure 67: False Bottom of Siphon System

\subsubsection{Moving Suction Cleaner (MSC)}

The moving suction cleaner is powered by the siphon in Figure 66, only the false bottom is replaced by an automatic pool cleaner. The siphon system is used in place of a pump used to power the automatic pool cleaner. The Lil Shark is an example of an automatic pool cleaner that may be attached to the siphon.

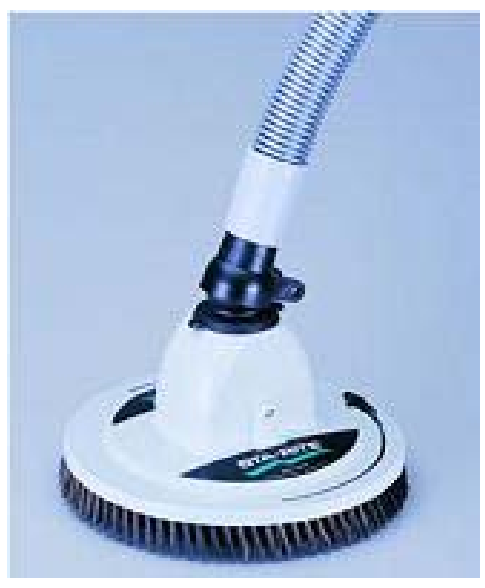

Figure 68: Lil Shark Automatic Pool Cleaner (1800Pools.com) 


\subsubsection{Sloped False Bottom (SFB)}

Considering the complication of the siphon system, a simpler idea is suggested. The sloped false bottom (Figure 69) consists of sloping walls that force settled solids to a concentrated area. This area is focused where the standpipe is located. The standpipe is removed and a hole with a radius corresponding to the standpipe is drilled in the sloped false bottom. A solenoid valve is placed at the end of the standpipe piping where the water enters the pre-existing filtering system. Since there is no electricity, a small battery will have to run the solenoid valve. The battery is charged by a solar panel. A timer is connected to the solenoid valve and battery to allow for timed cleaning cycles. Since all solids forced into a concentrated area around the standpipe hole, the flow rate from the standpipe system may be small.

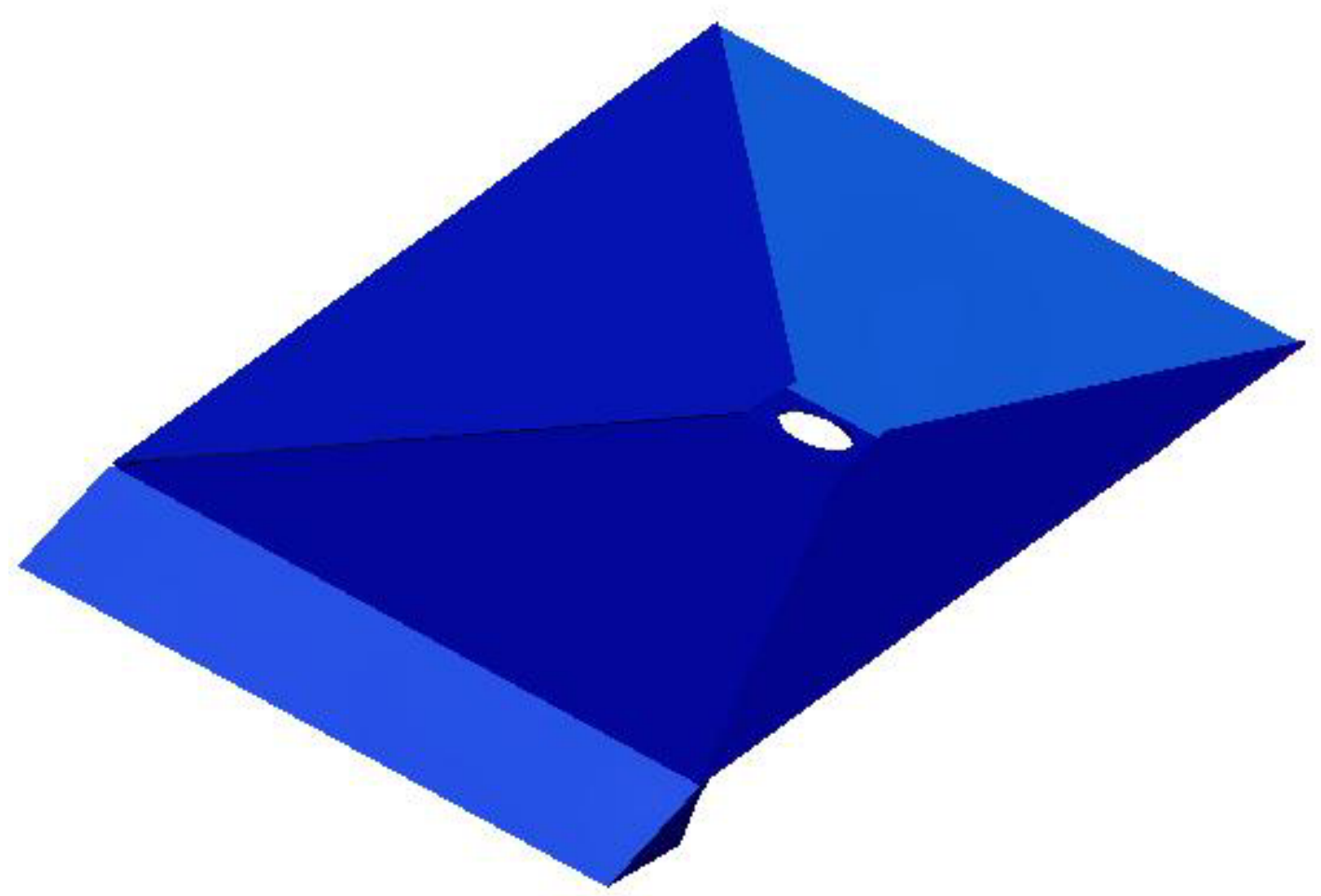

Figure 69: Sloped False Bottom

\subsubsection{Disturbance Filter (DF)}

This idea is inspired from a baffle system created by Boersen and Westers (1986). A series of baffles are aligned in the quiescent zone. The flow is diverted under and over top of the baffles. Flow near the bottom is increased to limit solid settling and force the solids to flow over 
the weir. The water and solids flowing over the weir then enter a bio-filter tub. The bio-filter tub is supported by the next raceway in series and has many holes drilled in the bottom to allow the filtered water to pass.

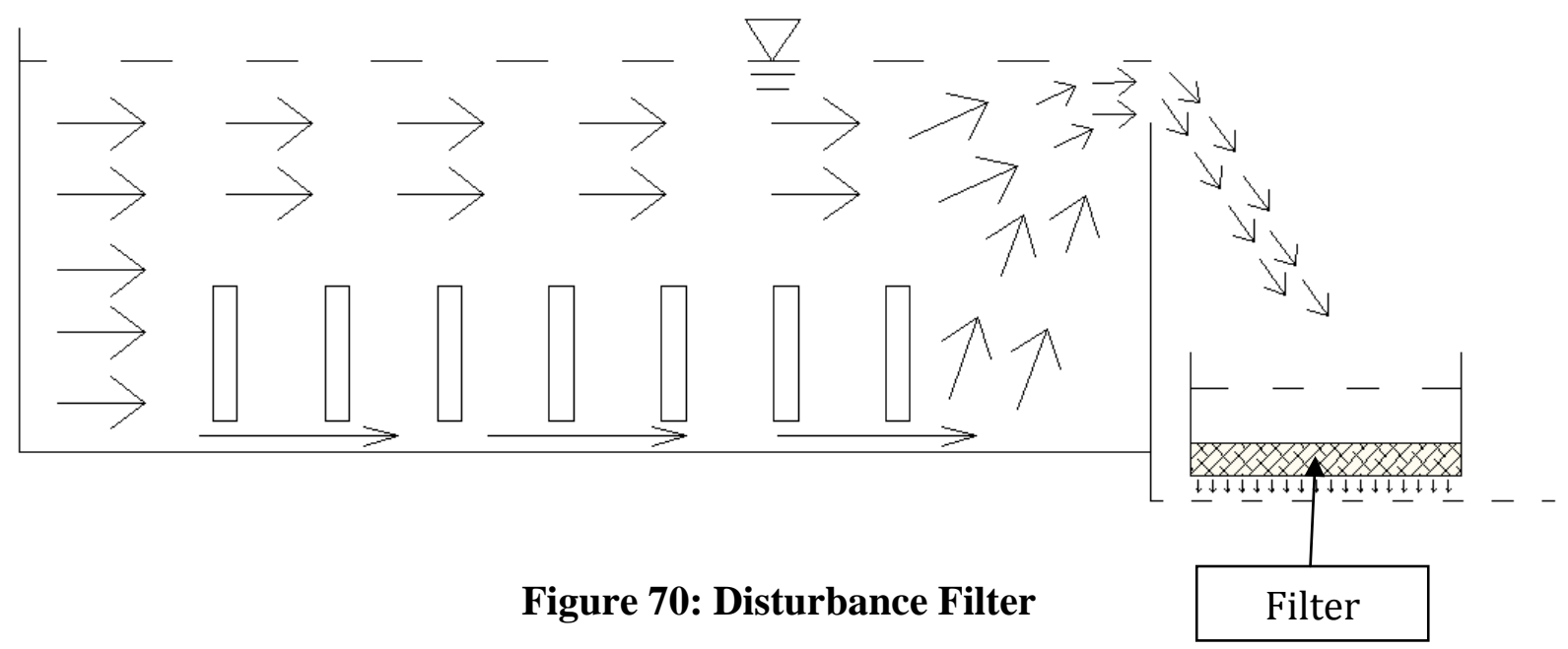

\subsubsection{Choosing a System}

Assuming the effectiveness of removing solids is the same for all ideas, a table is constructed (Table 9) to determine which ideas can best meet the basic requirements for installation at Dogwood Lake. Table 9 shows the rating of each idea with respect to each basic requirement. The rating is out of 5 , where 0 is the worst value and 5 is the best value for each requirement. The higher the rating, the better the idea is for installation at Dogwood Lake. Looking at the Total column in Table 9, the sloped false bottom (SFB) has the best rating. Based on Table 9, the sloped false bottom is the idea that is chosen for design and testing.

Table 9: Rating of Basic Requirements for Design Ideas

\begin{tabular}{|c|c|c|c|c|c|}
\hline Design & $\begin{array}{c}\text { Electricity } \\
\text { Required }\end{array}$ & $\begin{array}{c}\text { Easily } \\
\text { Installed/Removed }\end{array}$ & $\begin{array}{c}\text { Disruption of } \\
\text { Structure }\end{array}$ & Maintenance & Total \\
\hline SS & 5 & 2 & 5 & 4 & $\mathbf{1 6}$ \\
\hline MSC & 5 & 2 & 5 & 4 & $\mathbf{1 6}$ \\
\hline SFB & 4 & 4 & 5 & 4 & $\mathbf{1 7}$ \\
\hline DF & 5 & 2 & 3 & 2 & $\mathbf{1 2}$ \\
\hline
\end{tabular}

\subsubsection{Background and Critical Values of SFB}




\subsubsection{Determining Required Force to Move a Particle}

Using the Shields curve (Figure 71), the critical shear stress at the walls required to begin erosion of non-cohesive solids on the bottom of the quiescent zone can be found. The Shields curve relates a dimensionless mobility number (Equation 50) to a dimensionless grain size Reynolds number (Equation 51). In Equations 50-51,

$\Theta=\frac{\tau_{\text {Critical }}}{\left(\rho_{P}-\rho_{w}\right) g d}$

$R e^{*}=\frac{\sqrt{\frac{\tau_{\text {critical }}}{\rho_{w}}}}{v_{w}} d$

$\tau_{\text {Critical }}$ is the critical shear stress required to move a chosen particle, $\rho_{P}$ is the density of the chosen particle, $\rho_{w}$ is the density of water, $g$ is the acceleration due to gravity, $d$ is the diameter of the chosen particle, and $v_{w}$ is the kinematic viscosity of water. A grain size number relating to a mobility number greater than the Shields curve will result in movement of the chosen particle. Solving for the critical shear stress in Equation 51 and placing it into Equation 50 will result in Equation 52.

$\Theta_{\text {critical }}=\frac{R e^{* 2} v_{w}^{2} \rho_{w}}{\left(\rho_{P}-\rho_{w}\right) g d^{3}}$

Using a Microsoft Excel spreadsheet to guess $R e^{*}$ starting at 0.1 with increments of 0.1 in conjunction with Shields curve and Equation 52 will determine a critical mobility number. The critical shear stress (Equation 53) can then be found by placing the critical mobility number into Equation 50.

$\tau_{\text {critical }}=\theta_{\text {critical }}\left(\rho_{P}-\rho_{w}\right) g d$

The particles to be removed are assumed to be spherical. A diameter of $1 \mathrm{~mm}$ and a density of $1200 \mathrm{~kg} / \mathrm{m}^{3}$ are chosen for the characteristics of solid particles to be removed. This density is chosen based on the typical density of sinking fish food pellets (Juhani et al., 2001). This size is 
chosen as a reasonable median between the size of fish waste and the sinking fish feed. Using the method in the previous paragraph, a critical shear stress of approximately $0.17 \mathrm{~Pa}$ is found.

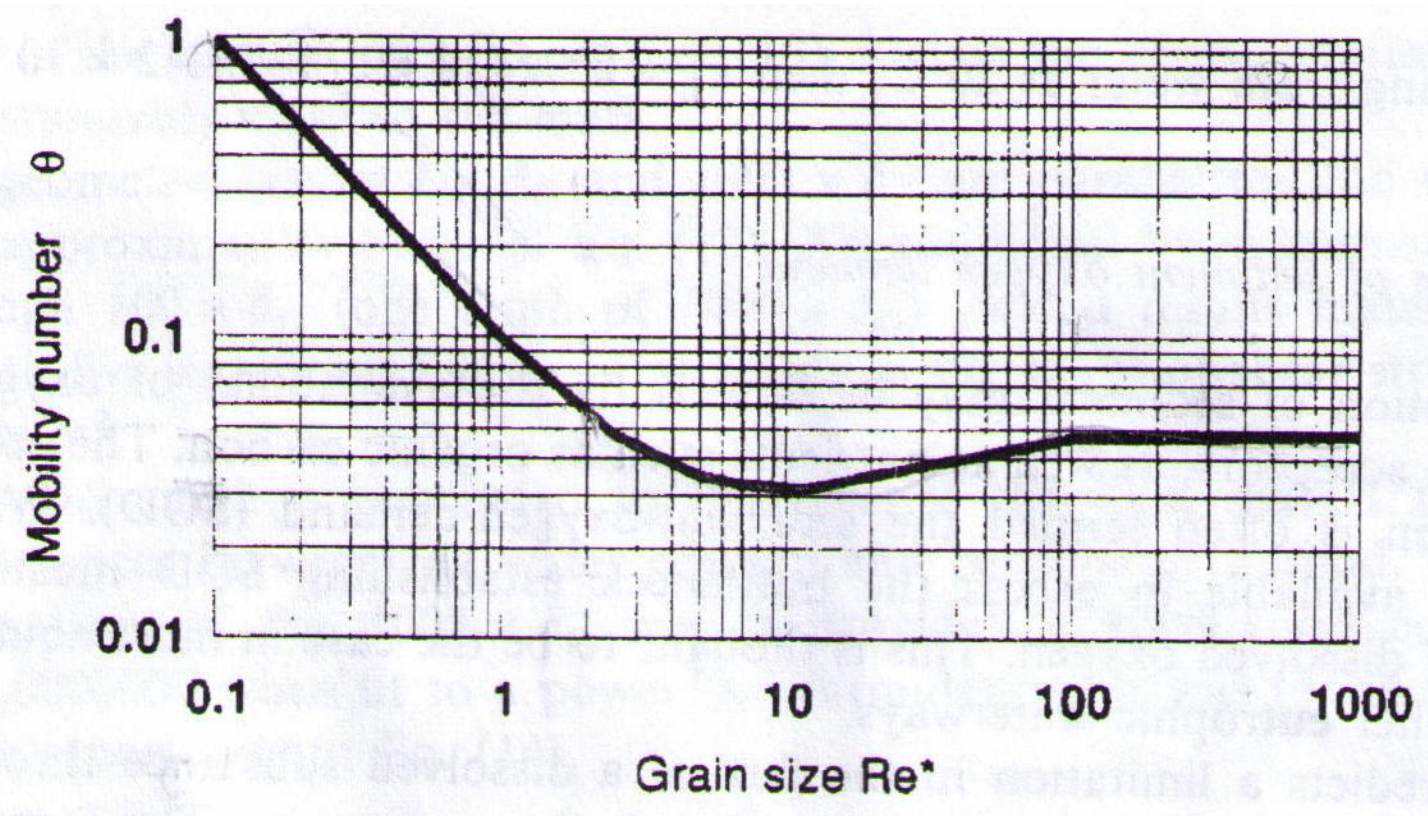

Figure 71: Shields Curve (Peterson, 1999)

\subsubsection{Critical Slope Required for SFB}

The main objective for this design is to determine the angles in which the sides should be sloped in order to force the settle solids to a concentration area.

\subsubsection{Critical Slope for Removing Particles with the Aid of Moving Water}

A free body diagram with forces in the downstream direction ( $x$-direction) is created for a $1 \mathrm{~mm}$ particle on a sloped surface (Figure 72). For simplicity, the friction of the particle on the surface is ignored, and will be compensated with a steeper slope than required. This results in the gravitational force and force of the moving water balancing the critical force required to start the movement of the particle. The sum of the forces in the $x$-direction can be seen in Equation 54 ,

$\sum F_{x}=\sin (\theta) F_{g}+\cos (\theta) F_{w}-F_{c r}$

where $F_{g}$ is the force on the particle due do gravity (Equation 55), 
$F_{g}=\forall_{P} \rho_{P} g$

$F_{w}$ is the force of the moving water (Equation 56),

$F_{w}=\rho_{w} u(y)^{2} A_{P}$

and $F_{c r}$ is the critical force required to move the particle (Equation 57).

$F_{c r}=\tau_{\text {critical }} A_{p}$

In Equation 56 and 57, $A_{p}$ is the cross sectional area of the particle and $\forall_{P}$ is the volume of the spherical particle.

Equation 58 is found by solving Equation 55 with the sum of forces equal to zero.

$\tau_{\text {critical }} A_{p}=\sin \left(\theta_{\text {critical }}\right) F_{g}-\cos \left(\theta_{\text {critical }}\right) F_{w}$

Using the critical shear stress found in Section 13.3.1 and an Excel spreadsheet used to guess values for $\theta_{\text {critical }}$ until the right side of the Equation 59 equals the left, a critical slope can be determined. The results show that the force of the water overpowers the force of gravity resulting in a zero slope required to move the particle. 


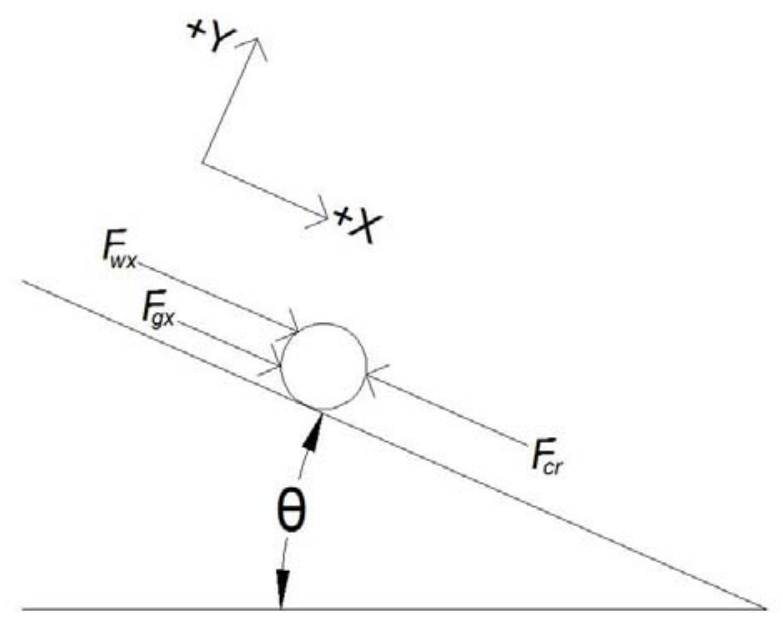

Figure 72: Free Body Diagram of a Particle on a Sloped Surface without Resistance of Water

\subsubsection{Critical Slope for Removing Particles with Resisting Water}

The only difference between Equation 59 and Equation 54 is the opposing force of the water on the particle (Figure 73). This force balance can be seen in Equation 59.

$\sum F_{x}=\sin (\theta) F_{g}-\cos (\theta) F_{w}-F_{c r}$

Equation 60 is found by setting the sum of forces in the $x$-direction equal to zero and solving for the critical shear force $\left(\tau_{\text {critical }} A_{p}\right)$.

$\tau_{\text {critical }} A_{p}=\sin \left(\theta_{\text {critical }}\right) F_{g}-\cos \left(\theta_{\text {critical }}\right) F_{w}$

Using an Excel spreadsheet and guessing values for $\theta_{\text {critical }}$ until the right hand side of the Equation 57 equals the left, results in a critical angle of approximately 7.4 degrees or a critical slope of $0.13(\tan (7.45))$. 


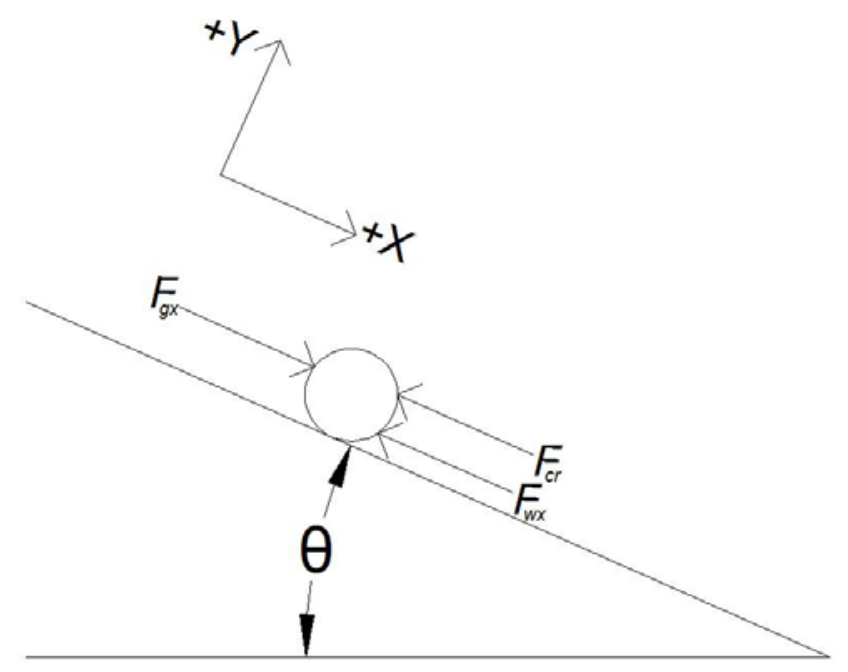

Figure 73: Free Body Diagram of Particle on a Sloped Surface with Resistance of Water

\subsubsection{Construction of SFB}

From Section 13.3.2, a critical slope of 0 is found to cause the chosen particle to move with the aid of the flow towards the standpipe hole. A critical slope of 0.13 is found (Section 13.3.4) to cause movement of the chosen particle when the flow resists the particle from moving towards the standpipe hole (note: particle is $1 \mathrm{~mm}$ in diameter with a density of $1200 \mathrm{~kg} / \mathrm{m}^{3}$ ). The SFB is designed with a bottom slope of approximately 0.052 to move particles toward the standpipe hole in the streamwise direction. The back slope is designed with a slope of approximately 0.78 . The side slopes are designed so that each side is made from one piece of material. In order to accomplish this, the sides must have a slight slope opposing the flow as well as toward the center of the quiescent zone. According to previous dimensions, the sides are forced to have a slope of approximately 0.65 towards the center of the false bottom and a slope of approximately 0.090 against the flow. The slope of the sides that oppose the streamwise flow is relatively small when compared to the slope toward the standpipe hole and therefore can be ignored.

The objective in construction of the SFB is to use three pieces of material to construct the entire false bottom. This is done to simplify fabrication and decrease cost. The material chosen must be malleable so that all slopes (excluding the sides) can be bent into shape using one piece of material. Also, the material must be able to resist the formation of rust. Aluminum sheet 
metal with a thickness of $0.63 \mathrm{~cm}$ is used for the material of the SFB. The sides of the SFB are then welded to the shaped bottom and back slope. The fabrication of the SFB is done by a local machining company (Wilsons Work in Morgantown, West Virginia). The finished SFB can be seen in Figure 74.

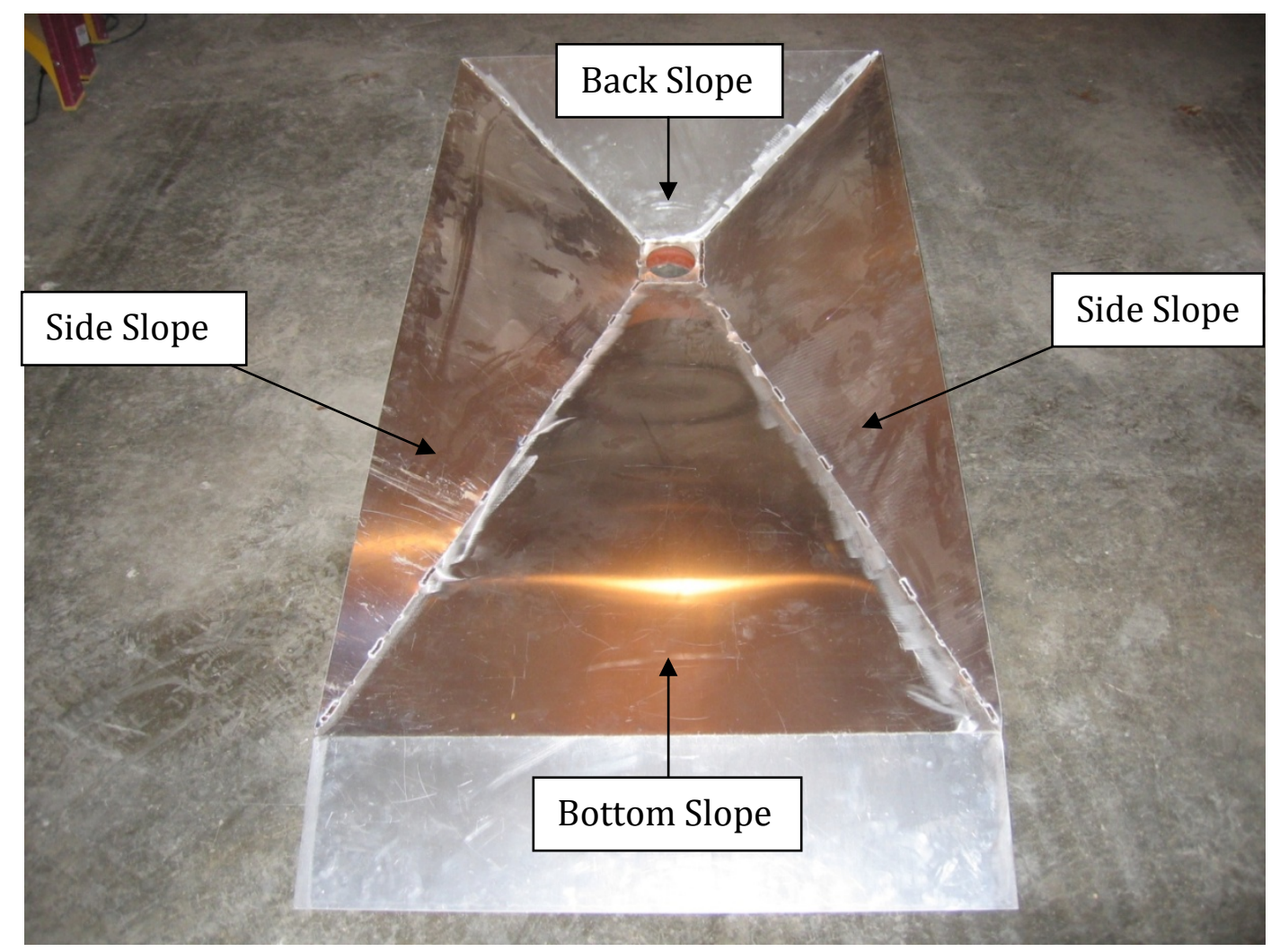

Figure 74: Final Construction of SFB

The next step in completing the SFB is to connect a solenoid valve and timing system to the end section of the standpipe system that leads to the pre-existing filtering bag at Dogwood Lake. The timing system consists of a simple fish feeding timer (Figure 75) connected to a 12 volt battery (Figure 76) and a $2.5 \mathrm{~cm}$ (diameter) solenoid valve (Figure 77). A 12 volt, 150 milliamp solar panel charger (Figure 78) is used to maintain charge in the battery. The timing system (battery, timer, solenoid valve) and solar panel are placed in individual waterproof protected cases (Figure 79). 


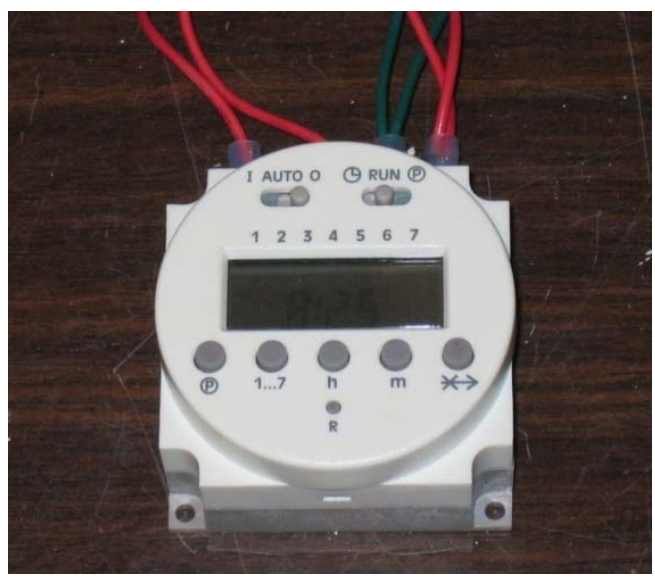

Figure 75: Fish Feeding Timer

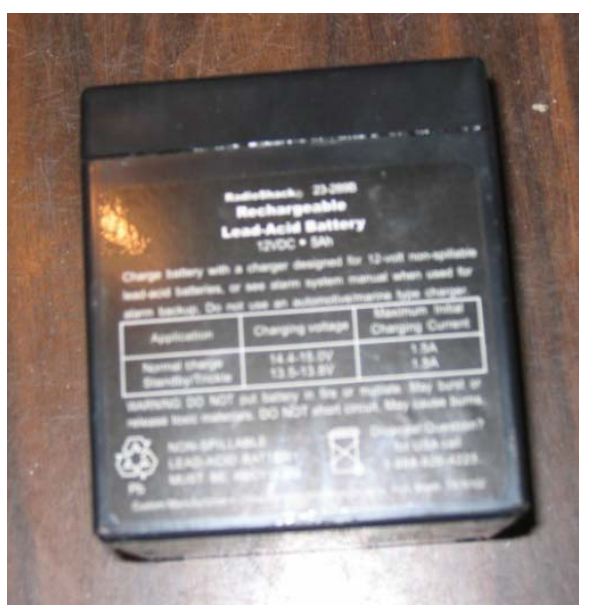

Figure 76: 12-V Battery

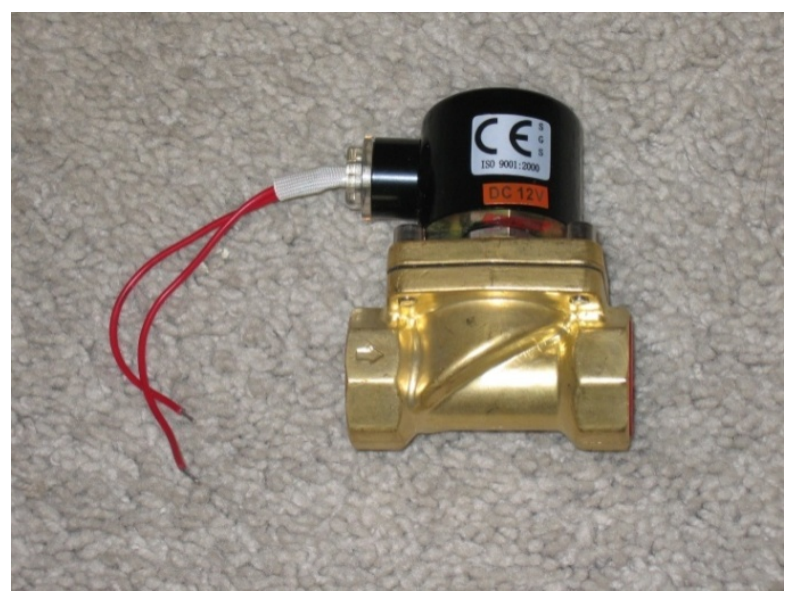

Figure 77: $2.5 \mathrm{~cm}$ Solenoid Valve 


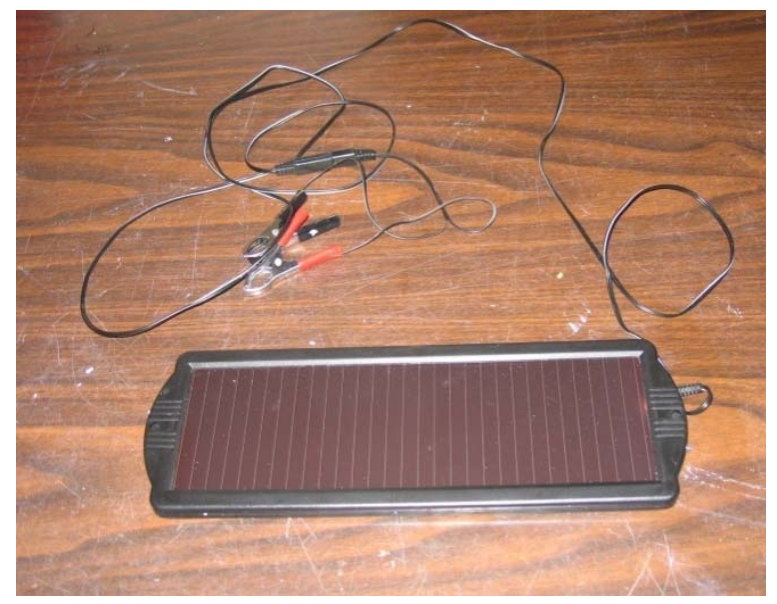

Figure 78: Solar Panel

Timing System

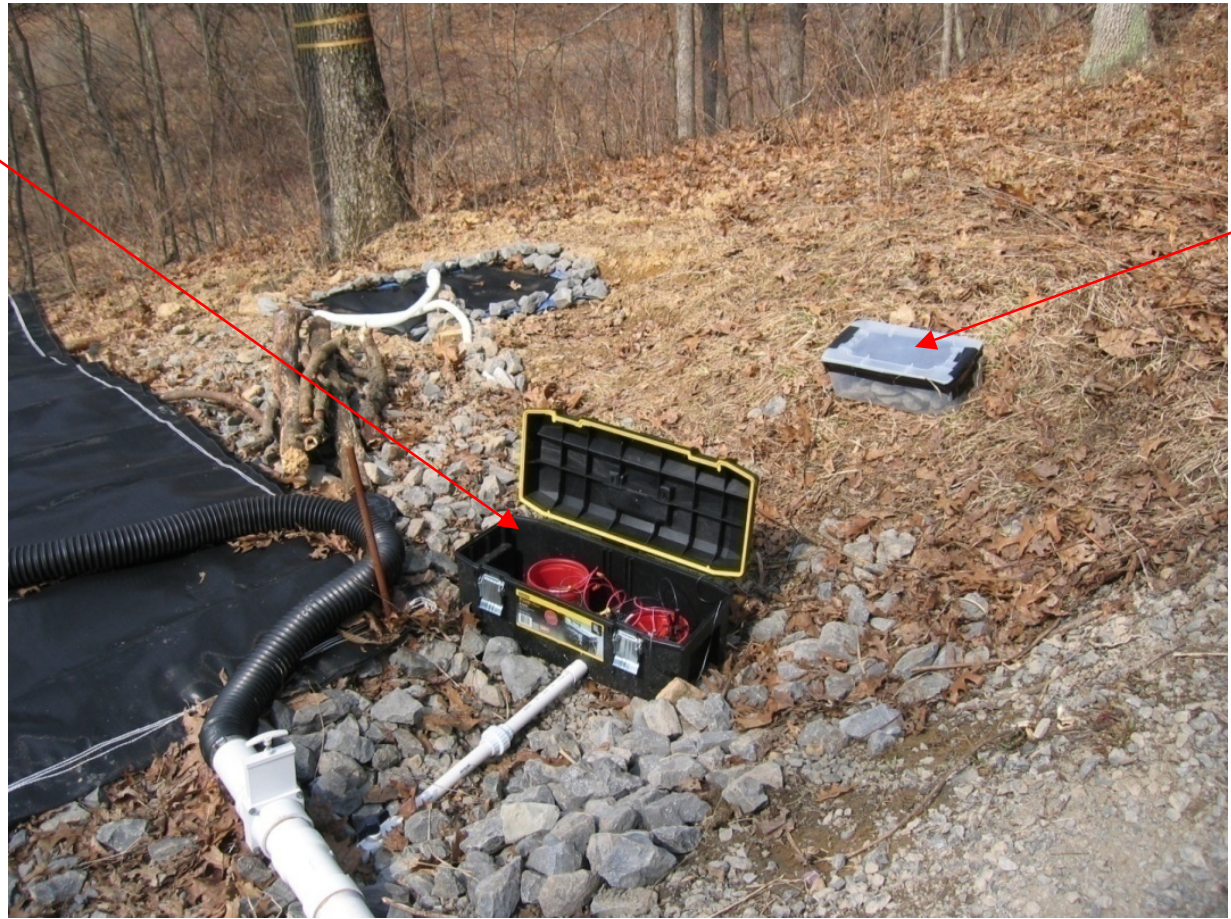

Figure 79: Waterproof Cases for Timing System and Solar Panel

\subsubsection{Tests and Results of SFB}

\subsubsection{SFB Test Description}

A test of the SFB was conducted to determine how well the system can clean the bottom of the quiescent zone. The timer was set to open the solenoid valve for 3 minutes every three hours. The system was left to run for 24 hours. 


\subsubsection{SFB Test Results}

The SFB system did not perform as expected. When returning to Dogwood Lake after the 24 hour period, the SFB was covered in sediment. Even the steepest slopes had little effect on movement of the solids toward the standpipe hole. Therefore, another system was tested.

\subsubsection{Choosing another System}

Table 9 is used to determine which of the systems has the next highest basic requirement rating. The siphon system (SS) and the moving suction cleaner (MSC) have the same requirement rating of 16 . Since the moving suction cleaner may cause particles to re-suspend and the SS has fewer moving parts, the SS is chosen for design.

\subsubsection{More Detail on the SS Design}

The basic idea of the siphon system is described in Section 13.1.1, but more detail on the system is required for a better understanding of the activation and deactivation of the cycle. Figures 80-81 show the major components required to activate and deactivate the siphon. Figures 82-84 represent the activation and deactivation of the siphon system.

The first three stages required to activate the siphon can be seen in Figure 82. In stage 1, water from the fill pipe fills the siphon encasement and the float rises. Stage 2 shows the water level reaching a critical height which allows the flap valve to open and the siphon to activate. This allows water from the siphon tub to be drained to the level seen in stage 3 . While the water is draining, the air in the siphon pipe is removed and some air is removed from the vertical pipe connecting to the siphon tub (VB-pipe), bridge pipe, and FB-pipe. In stage 3, the flap valve closes and the remaining air in the VB-pipe, bridge pipe, and FB-pipe is removed due to a pressure difference created by removing the air in the siphon pipe. This pressure difference allows water to move out of the quiescent zone (Figure 83) and to the filter system. Once the flap valve is closed, water from the fill pipe begins to fill the siphon tub again. Since the siphon tub begins to refill at stage 3 , the process starts over at stage 3 before the siphon is deactivated. Even though the cycle process has restarted, there is still one more stage required to deactivate the siphon from the quiescent zone. When enough water is siphoned out of quiescent zone, the water level drops (Figure 84) allowing air to enter into the stop hole and deactivate the siphon. The siphon activation/deactivation process is now complete and will repeat, beginning at stage 1 . 


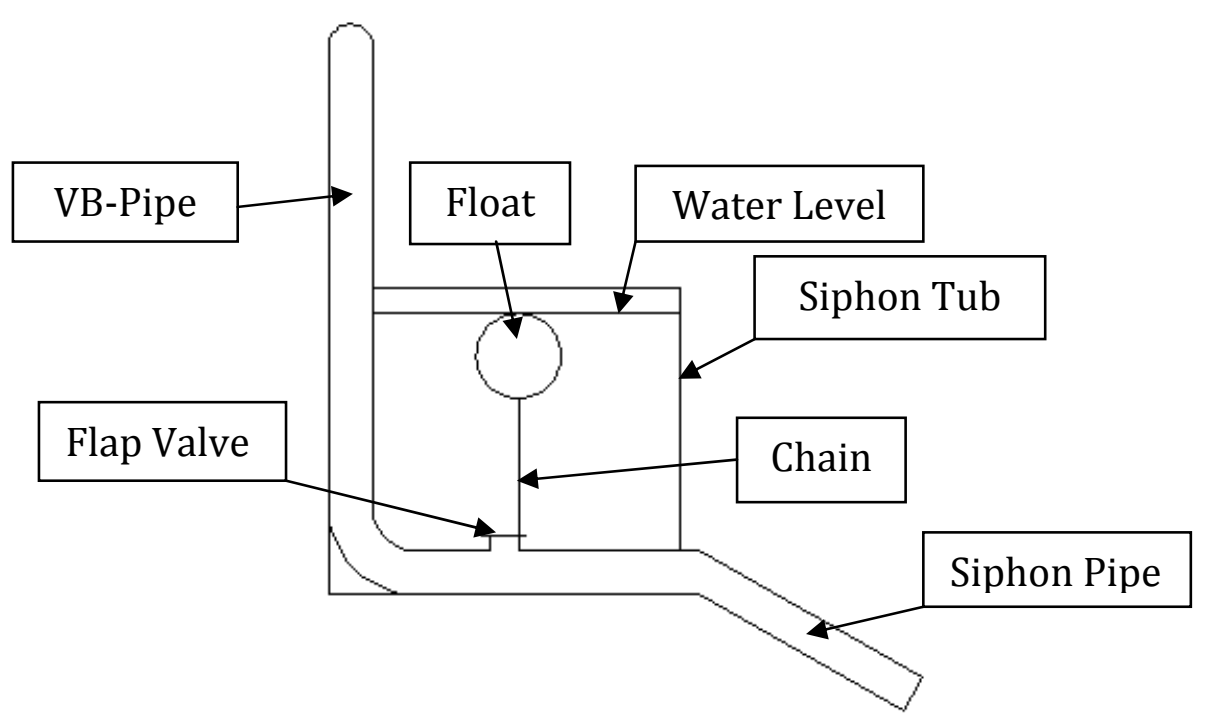

Figure 80: Siphon System Components

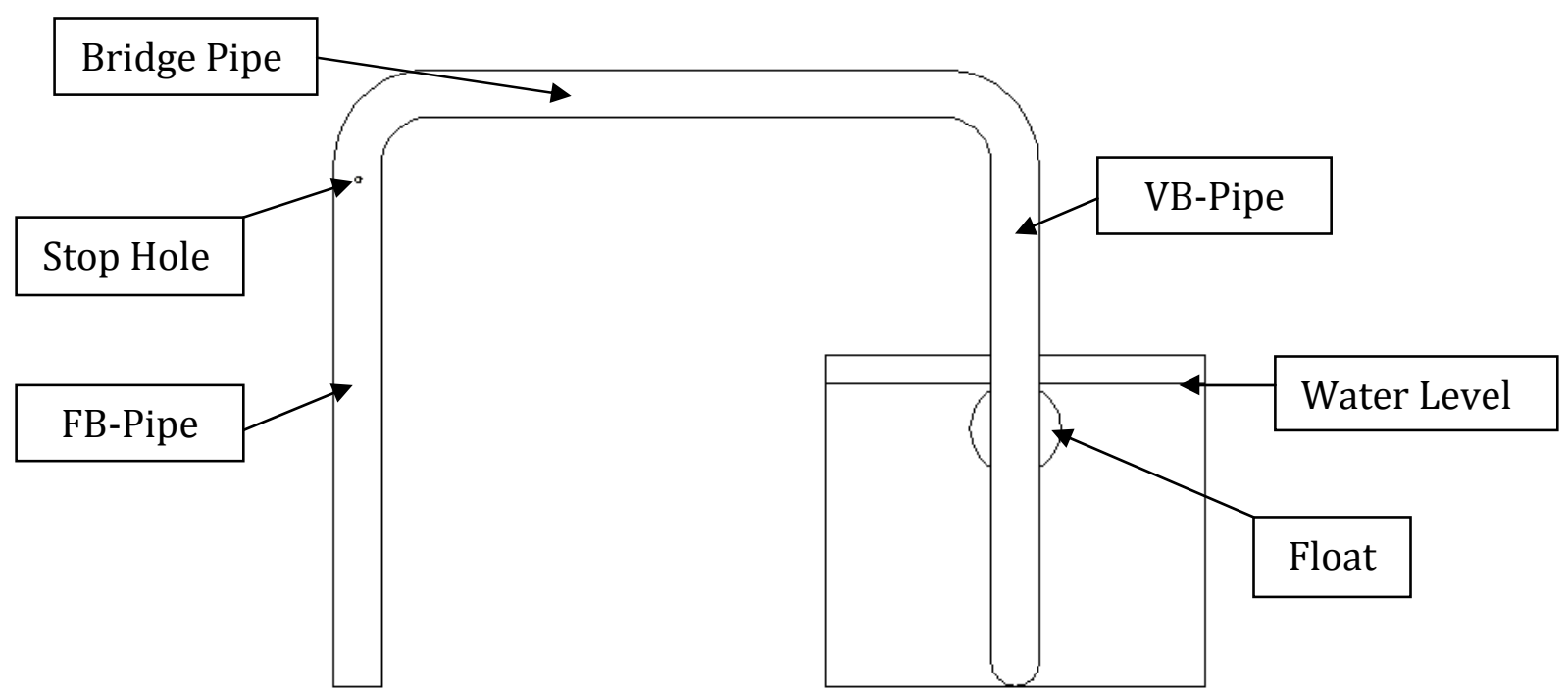

Figure 81: Piping from Siphon Tub to False Bottom 

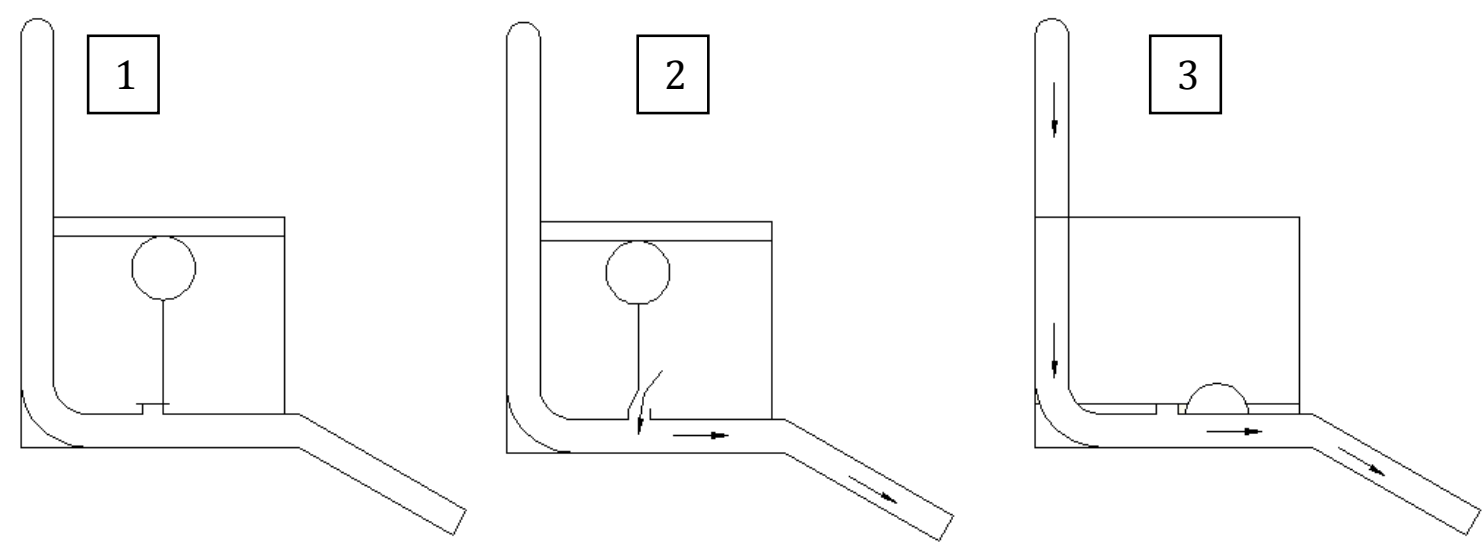

Figure 82: Siphon Activation Stages 1-3

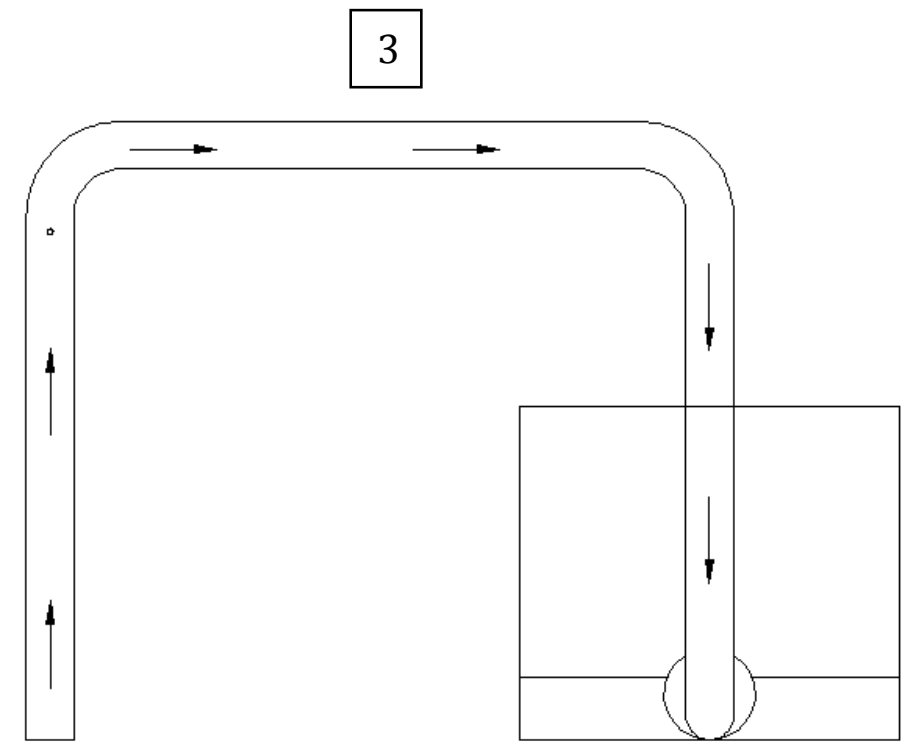

Figure 83: Side View of Siphon Activation (Stage 3) 


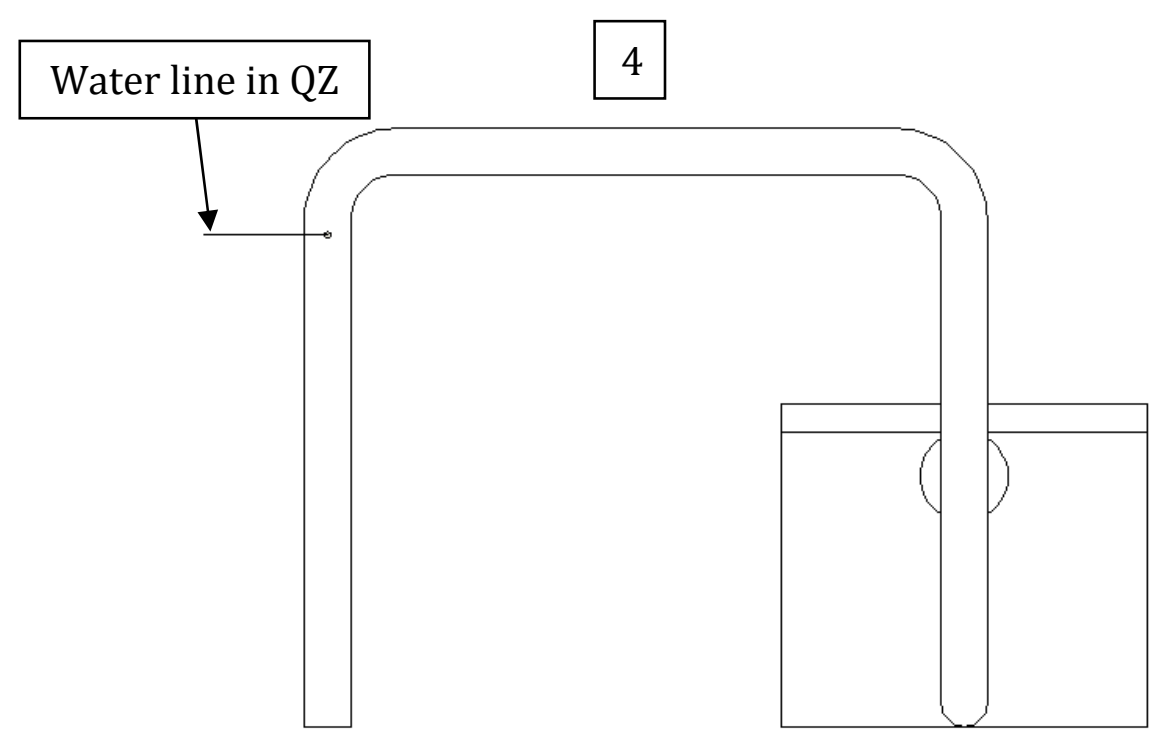

Figure 84: Side View of Siphon Deactivation (Stage 4)

While the siphon is active, suction is distributed into several areas covering the bottom of the quiescent zone. This is done via a false bottom. This false bottom contains several holes bored into the top surface. As the solid particles settle to the bottom of the quiescent zone, they will land in or around the collection holes. This allows for the solids to be removed when the siphon is active. A schematic of the false bottom can be seen in Figure 67.

\subsubsection{Background Required for Siphon System Design}

\subsubsection{Starting the Siphon}

The basic principle in starting the siphon is to remove the air from the piping system so the piping contains low pressure. This ensures that the hydrostatic pressure of the water in the FB-pipe exceeds the pressure in the piping. This creates a pressure difference causing the water in the FB-pipe to travel against the force of gravity. Once the water from the piping connected to the quiescent zone has traveled upward and then down towards the filtering system without letting air back into the piping system, the siphon is started. The only way to stop the siphon is to allow air back into the system. 


\subsubsection{Minimum Required Volume of Siphon Tub}

The siphon tub must hold enough water to allow the siphon pipe to completely fill with water. This is required so that the water in the siphon tub will remove all the air in the siphon pipe. The volume of the siphon pipe is then filled with the air that was once in the FB-pipe. The minimum required volume of the tub is dependent on dimensions chosen for the siphon pipe.

\subsubsection{Finding Flow Rate Required from SS to Cause Critical Shear Stress on Solids}

A $1 \mathrm{~mm}$ spherical particle with a density of $1200 \mathrm{~kg} / \mathrm{m}^{3}$ is used. It can be assumed that the particle is lying in the viscous sub-layer of the flow. Assuming the flow to be twodimensional, Equations 31-32 can be used while replacing $\overline{\tau_{w}}$ with $\tau_{\text {critical }}$ found by using Shields curve (Figure 71). Assuming that the flow near the bottom is slow enough to be ignored, a critical velocity $\left(V_{\text {suction critical }}\right)$ at the height of the particle $(1 \mathrm{~mm})$ is required to create a critical shear stress at the wall. Due to the small diameter of the particle, the critical shear stress at the wall is assumed to cause the particle to move. The mean velocity in Equation 32 then becomes $V_{\text {suction critical }}$ (Equation 62).

$V_{\text {suction critical }}=\frac{\tau_{\text {critical }}}{\rho_{w} v_{w}} Z$

Considering the holes in the false bottom to be two-dimensional sinks acting at a point, Equation 63 can be used. In Equation 63,

$V_{\text {suction } \text { critical }}=\frac{\left(\frac{Q_{\text {critical }}}{\text { hole }}\right)}{2 \pi r^{2}}$

$V_{\text {suction critical }}$ is normally the radial velocity created at a radial distance $\mathrm{r}$ from a twodimensional point sink, but is used as the critical velocity to create the critical shear stress at the wall and begin movement of a particle a radial distance $r$ from the center of a collection hole in the false bottom. Solving for $Q_{\text {critical }} /$ hole in Equation 63, results in Equation 64. 
$\frac{Q_{\text {critical }}}{\text { hole }}=V_{\text {suction critical }}\left(2 \pi r^{2}\right)$

\subsubsection{Estimating Flow Rate that the Siphon System can Provide}

The flow rate that the siphon system can provide depends on the dimension of the piping system and vertical elevation that the water falls to the filter system. Using the Bernoulli equation which ignores energy losses, an approximation of the flow rate can be found ignoring losses in energy to viscosity and bends in the pipe. Evaluating the Bernoulli equation at the beginning (point 1 ) and end (point 2) of the siphon pipe will allow the velocity at point 2 to be calculated $\left(V_{\text {exit }}\right)$ (Figure 85).

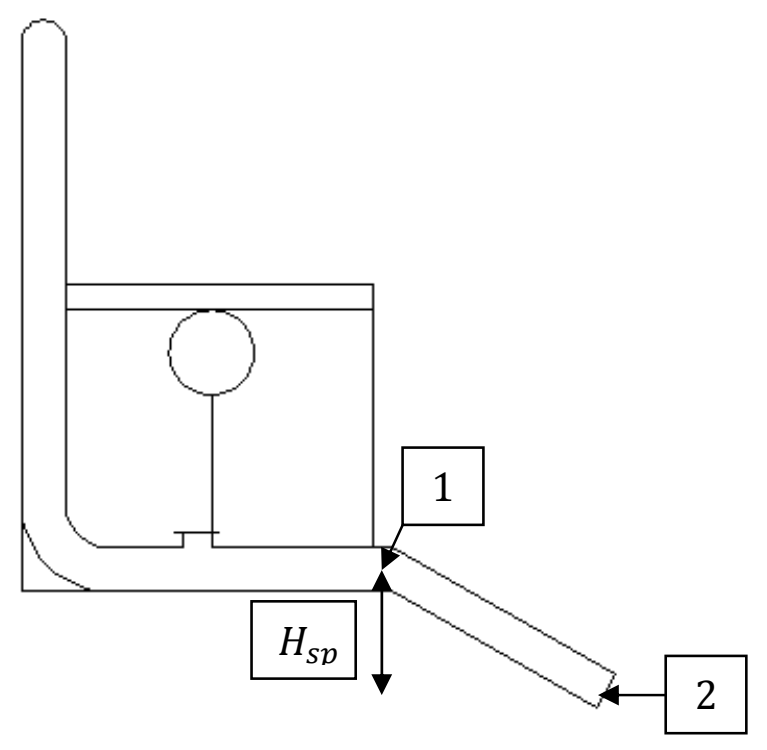

Figure 85: Finding Exit Velocity

For simplicity, points 1 and 2 are assumed to be at atmospheric pressure and the vertical distance between them is $H_{s p}$. Equation 65 can then be used to approximate a velocity at the exit of the siphon pipe.

$V_{\text {exit }}=\sqrt{2 g H_{s p}}$ 
It should be noted that the height of the VB-pipe leading from the siphon tub to the bridge pipe is ignored when estimating the volumetric flow rate that the siphon can provide. Knowing the diameter of the siphon pipe, the volumetric flow rate can be found (Equation 66).

$$
Q=V_{\text {exit }}\left(\frac{\pi d^{2}}{4}\right)
$$

\subsubsection{Design of Siphon System}

\subsubsection{False Bottom}

The false bottom is to be designed so that particles settling on the bottom are removed using suction from the siphon. From observation of the coverage radius on the settled particles provided by the current standpipe system, a conclusion is drawn that the current flow rate provided by the standpipe system may not be sufficient to clean the entire bottom of the quiescent zone. For this reason two separate false bottoms connected to two separate siphon systems will be used to increase the coverage radius. The objective is to double the flow rate of the current standpipe system. Since the current stand pipe system uses a pipe diameter of $7.6 \mathrm{~cm}$ and has an approximate drop of 5 meters, each siphon system will be designed with the same parameters.

For each false bottom, the width and length is chosen to be approximately $81 \mathrm{~cm}$ and 89 $\mathrm{cm}$, respectively. The width and total length of both false bottoms are smaller than the width and length of the quiescent zone to assure that they fit between the walls of the quiescent zone. In order to determine the number of holes to be drilled in the false bottom, the volumetric flow rate that the siphon can provide must be determined before continuing. Before the flow rate is approximated from Equation 63, the vertical drop of siphon tub to the filter system and diameter of the siphon pipe must be chosen. A vertical drop $\left(H_{s p}\right)$ and siphon pipe diameter are chosen to be the same as the standpipe system (vertical drop from quiescent zone A4 to the filtering system) at approximately $5 \mathrm{~m}$, and $7.6 \mathrm{~cm}$ respectively. Equation 65 can now be used to determine $V_{\text {exit }}$. The exit velocity $(9.9 \mathrm{~m} / \mathrm{s})$ is found and used in Equation 66 to approximate the 
volumetric flow rate created by the siphon. The volumetric flow rate created by the siphon is approximated to be $0.045 \mathrm{~m}^{3} / \mathrm{s}$.

Next, the position, number, and size of the holes that distribute the flow throughout the top surface of the false bottom were determined. Knowing that the holes were to be drilled through a plastic material (used for the top surface of the false bottom), small diameter holes (approximately $0.6 \mathrm{~cm}$ in diameter) were chosen to limit cracking during the drilling process. The number of holes and coverage radius was determined using an AutoCAD drawing (Figure 86). The drawing provided visualization of the total coverage area on the top surface of the false bottom. The total coverage area created by the collection holes in Figure 86 does leave areas that will not be cleaned. These small areas of un-cleaned surface are sacrificed in order to limit the overlap of coverage radii. Equation 64 can be used to determine the required flow rate from the siphon system, knowing the number of holes and the individual coverage radii required. The required flow rate is then compared to the approximated flow rate that siphon can provide $(0.045$ $\mathrm{m}^{3} / \mathrm{s}$ ). Forty holes each possess a coverage radius of $8.9 \mathrm{~cm}$ which is determined to clean the majority of the surface of the false bottom. The required flow rate to provide this cleaning radius to each collection hole is approximated to be $0.034 \mathrm{~m}^{3} / \mathrm{s}$ (using Equation 64). Comparing the critical flow rate $\left(.045 \mathrm{~m}^{3} / \mathrm{s}\right)$ to the required $\left(0.034 \mathrm{~m}^{3} / \mathrm{s}\right)$, shows that the siphon should provide the flow rate necessary to clean the majority of the false bottom. 


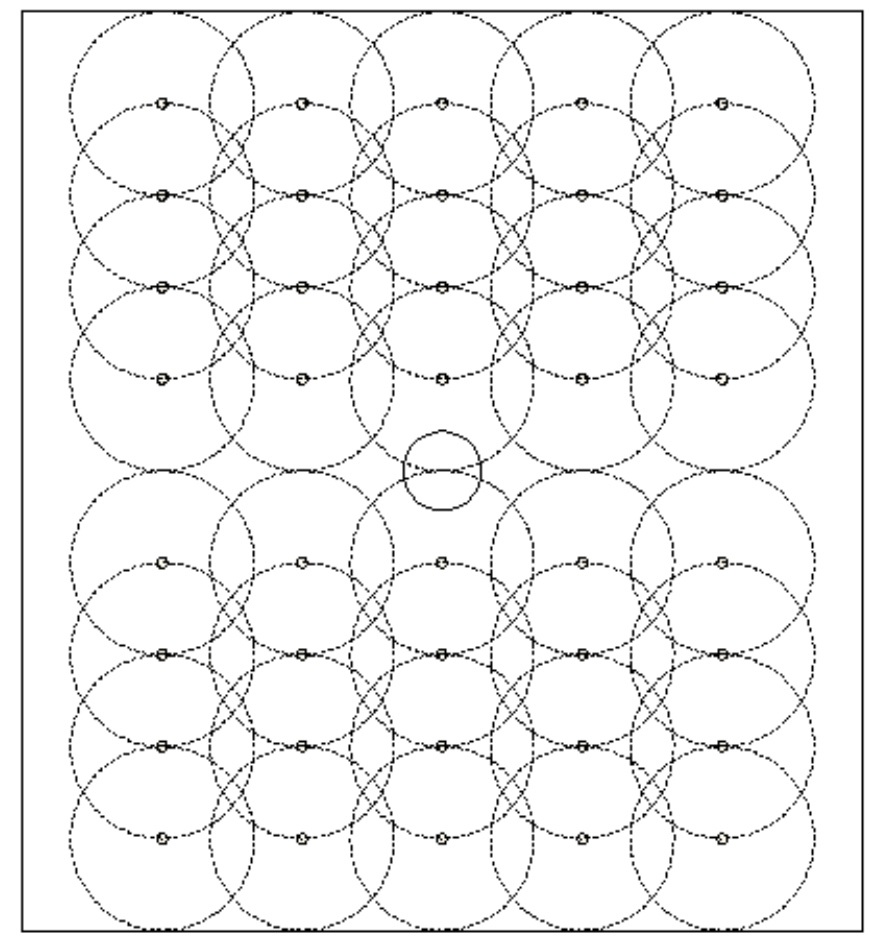

Figure 86: Determining Coverage Radius and Number of Holes Required for False Bottom

\subsubsection{Siphon Tub}

Now that the vertical length of the pipe exiting the siphon tub is approximated to be $5 \mathrm{~m}$ and $7.6 \mathrm{~cm}$ in diameter, the ground slope of the location where siphon test is to be run must be approximated. The slope is approximated to be 1.2 at the location of the siphon test. A slope of 1.2 results in a total siphon pipe length of approximately 5.5 meters. Now that the total siphon pipe length and diameter are known, the volume of the siphon pipe can be determined. The minimum required volume of the siphon tub is equal to the volume of the siphon pipe. A volume of $0.025 \mathrm{~cm}^{3}$ is found as an approximation of this volume. A trip to a local retail store provided a tub with a volume of approximately $0.11 \mathrm{~m}^{3}$.

\subsubsection{Construction of Siphon System}

\subsubsection{False Bottom Construction}

The surface of the false bottom should be smooth and the volume that the false bottom consumes should be minimized. In order to minimize this volume, a series of $2.5 \mathrm{~cm}$ in diameter PVC pipes were constructed. To create a smooth surface, a sheet of Plexiglas is connected to the 
top surfaces of the pipes. At the center of each false bottom a connection for the FB-pipe is placed. Holes are then drilled through the Plexiglas and PVC at the desired location. One section of the finished false bottom can be seen in Figure 87.

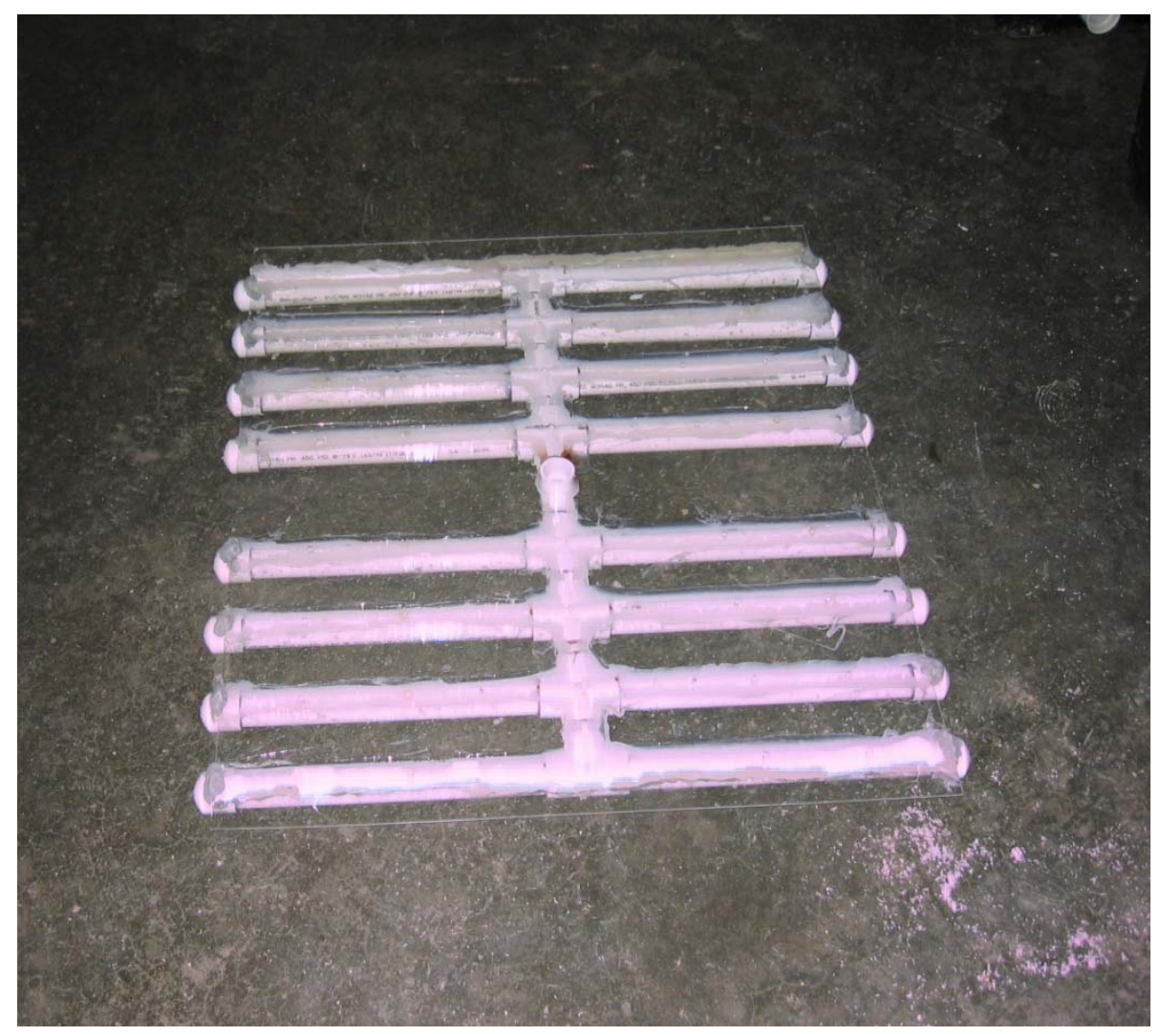

Figure 87: Finished False Bottom

\subsubsection{Siphon Tub Construction}

The siphon tub used is made from plastic. A hole drilled into the side of the tub allows the siphon pipe to connect to the siphon tub. Two PVC couplings are used on either side of the drilled hole to limit the movement of the pipe. A short piece of PVC pipe is connected to the coupling inside the tub. The short piece of PVC allows a PVC tee to connect to the coupling. The vertical section of the PVC tee allows the flap valve system to be connected. Another short piece of PVC pipe is connected to the opposite side of the PVC tee. The PVC is then angled upward $90^{\circ}$ in order to connect to the VB-pipe. A threaded connector is added to the vertical tee section in the siphon tub and the flap valve system (Figure 88) is threaded onto the connector. The inlet of the flap valve system is approximately $5.7 \mathrm{~cm}$ in diameter. It was noticed that a problem may occur since the inlet of the flap valve is smaller than the diameter of the siphon 
pipe. It is necessary that the siphon pipe is completely filled with water when the flap valve is opened. If the inlet diameter is smaller than the pipe diameter, this may not occur. A pipe reducer is used to decrease the diameter of the siphon pipe to approximately $3.8 \mathrm{~cm}$. This results in the piping system remaining constant at $7.6 \mathrm{~cm}$ from the false bottom to the pipe reducer and then reducing the siphon pipe to approximately $3.8 \mathrm{~cm}$. The flow rate should remain approximately the same (ignoring energy losses) since the piping carrying water to the reduced siphon pipe is $7.6 \mathrm{~cm}$ in diameter. The tub volume approximation in Section 13.9.2 becomes more conservative since the volume in piping leading to the filtering system is decreased; however, due to the elevation of the flap valve system, the volume used to fill the siphon pipe is reduced. Since the actual volume of the tub is approximately 4.4 times larger than the minimum volume required, the height of the flap valve does not cause a problem. The reduction in the siphon pipe diameter may cause a problem if the volume of the air in the piping leading to the false bottom is larger than the volume of the siphon pipe. This must be considered when installing the system. Minimizing the lengths of the bridge pipe, VB-pipe, and FB pipe is critical when installing the siphon system.

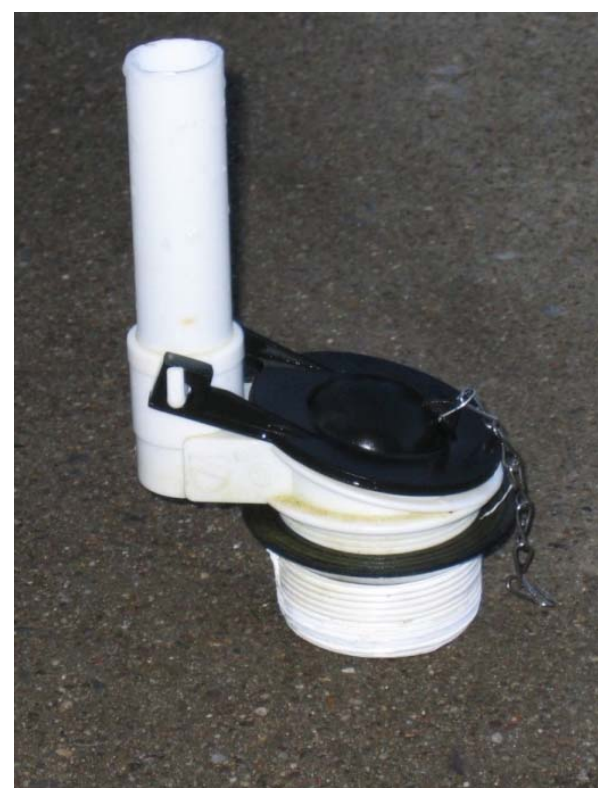

Figure 88: Flap Valve System 


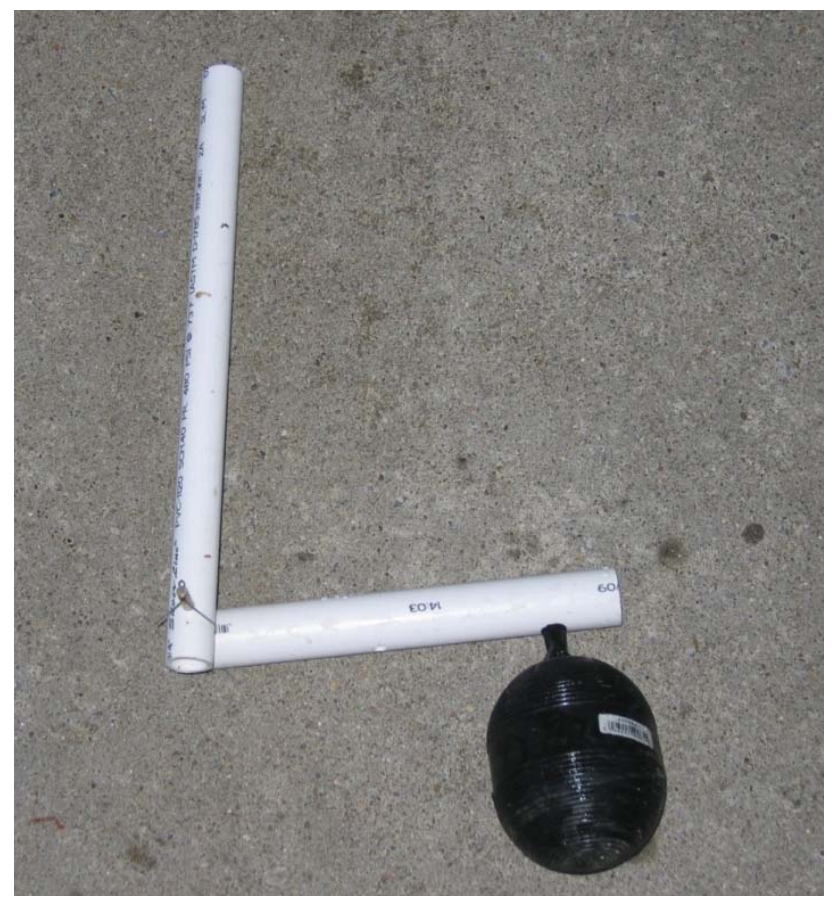

Figure 89: Buoyancy System

A buoyancy system (Figure 89) is connected to the flap valve system. The buoyancy system is made from a toilet float and a short section of PVC. A chain is then connected to the PVC section supporting the float. The location of the chain on the PVC is determined by trial.

\subsubsection{Preliminary Tests on Siphon Tub}

Tests were run on the siphon tub (Figure 90) to determine how well the buoyancy system opens the flap valve when set to open a completely filled siphon tub. It was determined that the float did not create enough buoyancy to open the flap valve. The flap valve opened and closed, but did not remain open during numerous tests. Several toilet floats were then added to the buoyancy system. The additional floats did not cause the flap valve system to completely open. A $90^{\circ}$ angle in the beginning section of the siphon pipe was eventually added to allow water to enter when the flap valve fluctuated from the open and closed position. This caused water to fill the vertical section of the newly added $90^{\circ}$ piping section when the flap valve cycled from the open and closed position. When water filled the $90^{\circ}$ piping section, the flap valve would close and the tub would fill again. When the chain became taut again, the water pressure created in the vertical section of the $90^{\circ}$ angle allowed the flap valve to open. The vertical height of the $90^{\circ}$ angle was calibrated each time the siphon system was tested. 


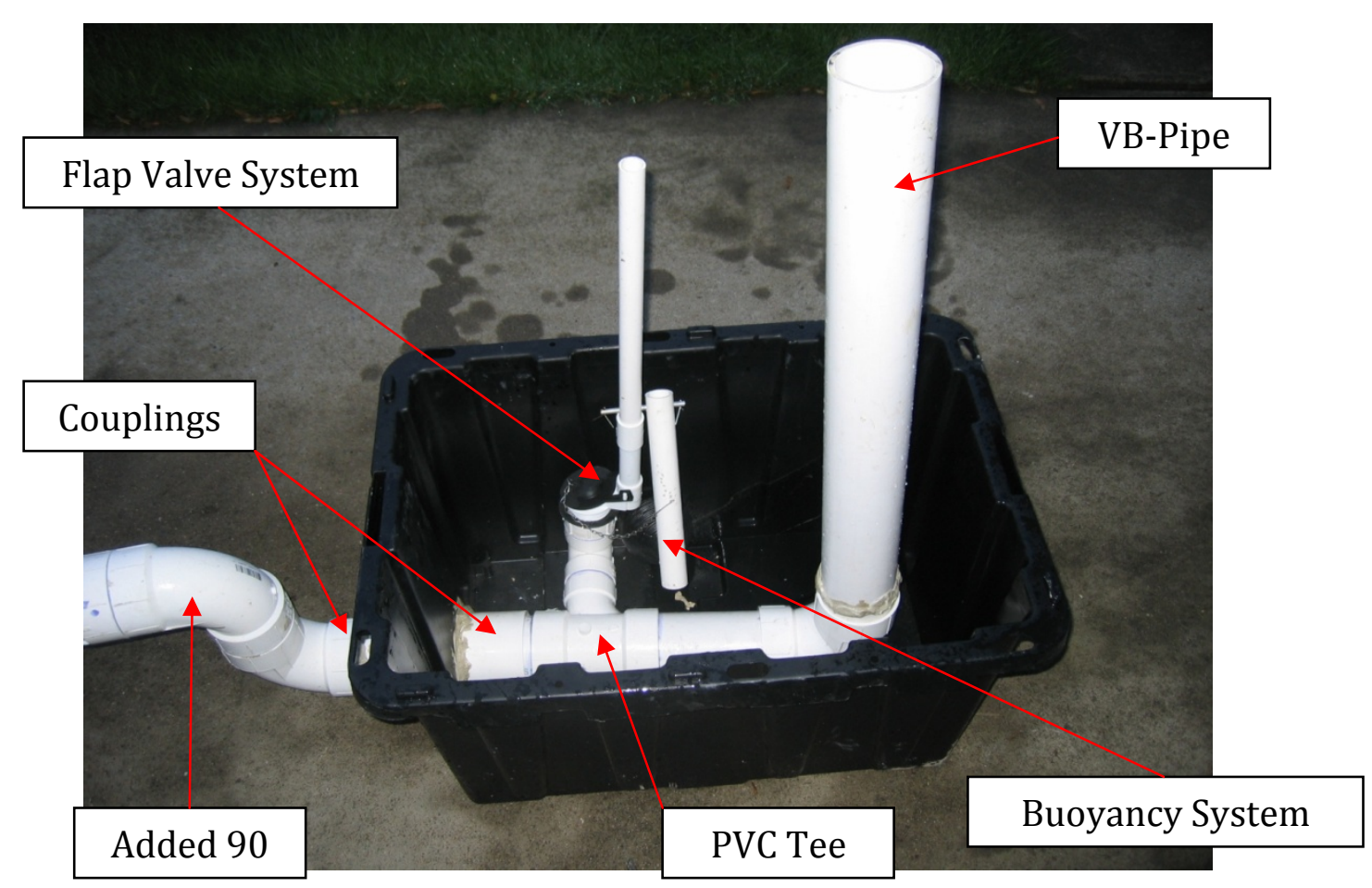

Figure 90: Finished Siphon Tub

\subsubsection{Siphon System Tests}

A test of the system was conducted to determine how well the system can remove solids from the bottom of the quiescent zone. The test is preformed on the most downstream quiescent zone at Dogwood Lake. Placing the false bottom into the quiescent zone one day before the tests were conducted allowed solids to completely cover the top surface of the false bottoms. The setup of Test 2 (Figure 92) is identical to Test 1, other than the difference in false bottoms.

\subsubsection{Siphon System Test 1 Results}

With both siphons fully activated for approximately 10 minutes, small areas around the holes (approximately $0.63 \mathrm{~cm}$ in diameter) were cleaned; however, the rest of the surface remained completely covered. The siphons were manually deactivated by drilling two small holes into the bridge pipe to allow air into the system. Manual deactivation was necessary since the flow rate was not large enough to cause a drop in the water level in the quiescent zone. The turbidity of the water was high and a photo of the false bottoms through the water was not clear.

It was concluded that the loss of energy due to the complicated geometry of the false bottom may be causing the flow rate to diminish. Also, the diameter of each hole in the false bottom is very small and may be causing large losses of energy. 


\subsubsection{New False Bottom}

With the results from Test 1, a new type of false bottom was designed with a simple geometry and larger inlet holes on the top surface (Figure 91). Eight additional holes are added to the new false bottom. The additional holes allowed smaller spacing between holes and the required flow rate of approximately $0.045 \mathrm{~m}^{3} / \mathrm{s}$ to provide the same coverage radius as mentioned in Section 13.9.1. The new false bottom is made using a $3.8 \mathrm{~cm}$ thick wood frame and a sheet of $0.63 \mathrm{~cm}$ thick Plexiglas on both the top and bottom surfaces. Holes are drilled through the Plexiglas and the wood frame. Plumbers putty is placed on the edges of the wood frame where the Plexiglas lays. Hexagonal bolts are placed in the drilled holes to hold the Plexiglas and wood frame in place. Hexagonal nuts are then threaded on the bolts and tightened to create a seal between the Plexiglas and wood frame.

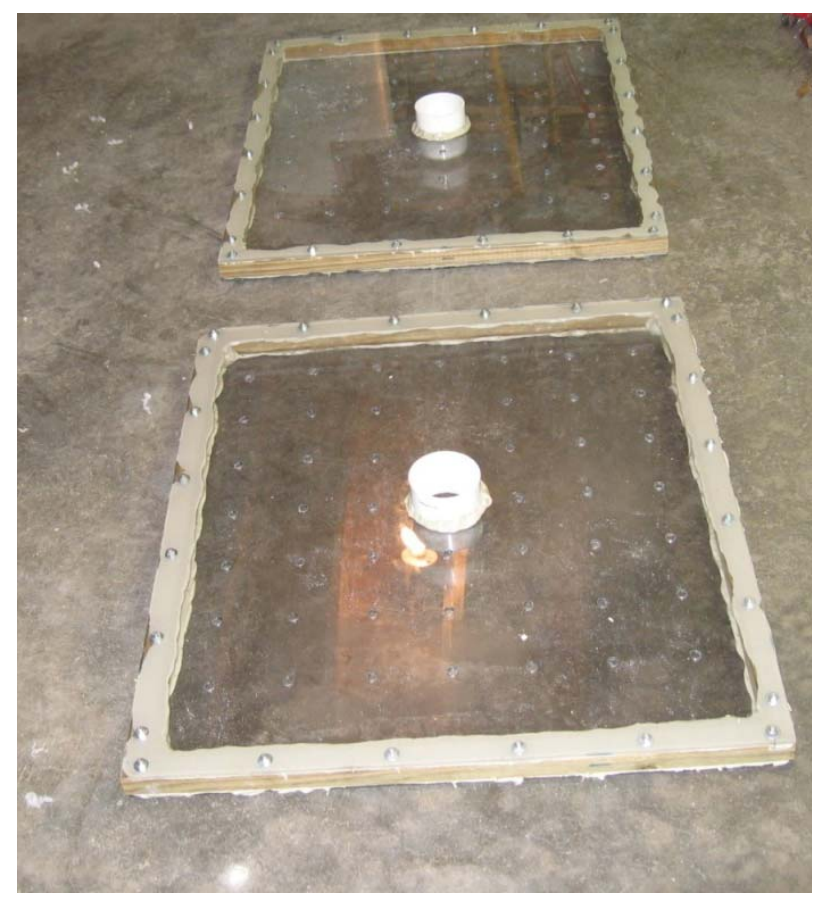

Figure 91: New False Bottom

\subsubsection{Siphon System Test 2 Results}

Test 2 was run for approximately 4 minutes; however, most of the cleaning was accomplished in the first minute. The setup of Test 2 can be seen in Figure 92. Figure 93 shows the outlet of the siphons while both siphons were active. Again, the siphons were manually deactivated via the holes drilled in the bridge pipes. The flow rate was sufficient to drop the 
water level past the weir, but the incoming downstream flow balanced the water level at this height. The results from Test 2 were similar to Test 1 . The cleaning diameter around each hole appeared to increase to approximately $2.5 \mathrm{~cm}$ (Figure 94). An increase in flow rate was noticed by observing the water exiting the system at the bottom of the hill when compared to Test 1 . A flow rate for Test 2 of $0.006 \mathrm{~m}^{3} / \mathrm{s}$ was estimated by the height of the water jet at the exit and the cross sectional area of the siphon pipe.

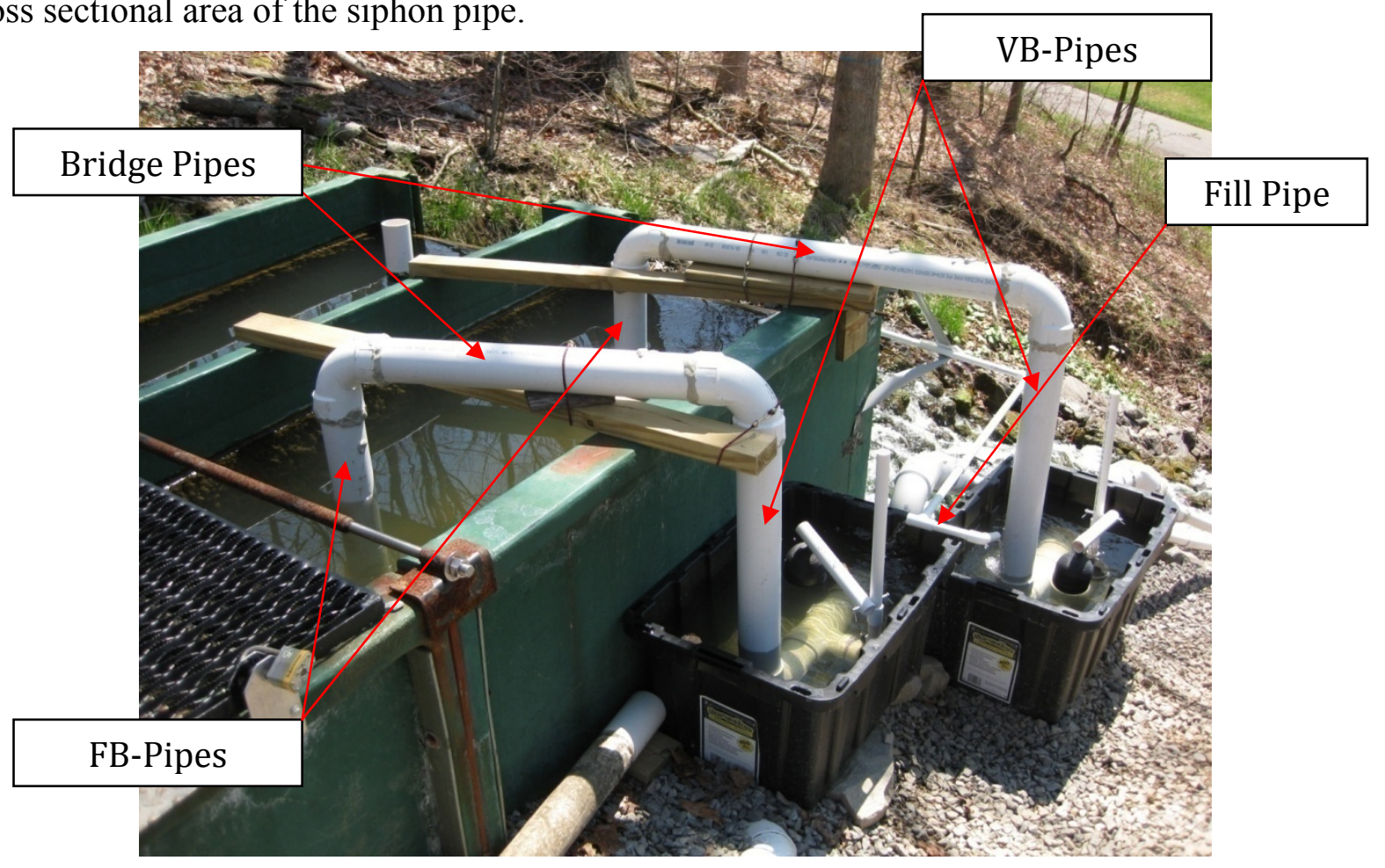

Figure 92: Siphon System Test 2 Setup 


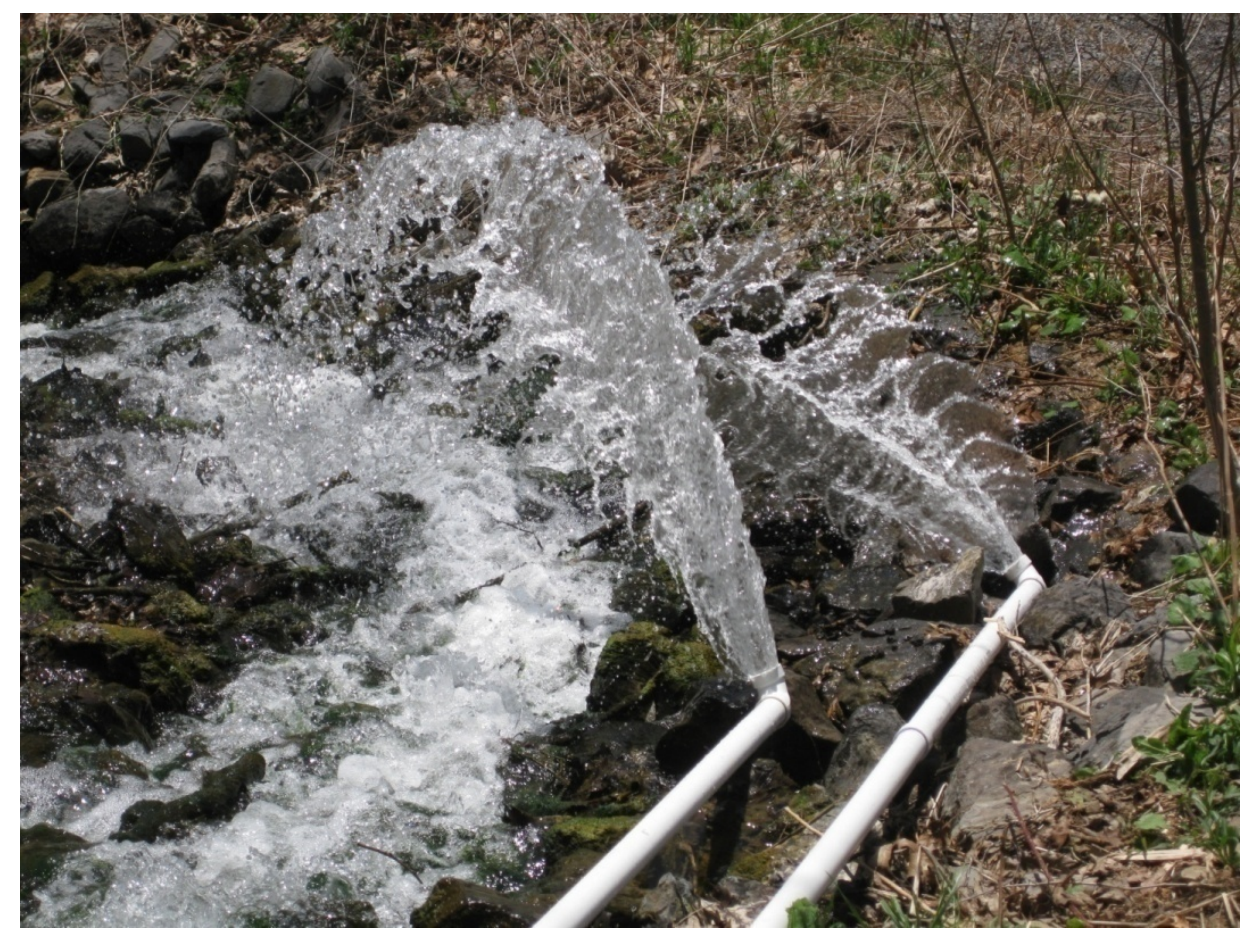

Figure 93: Siphon Outlet

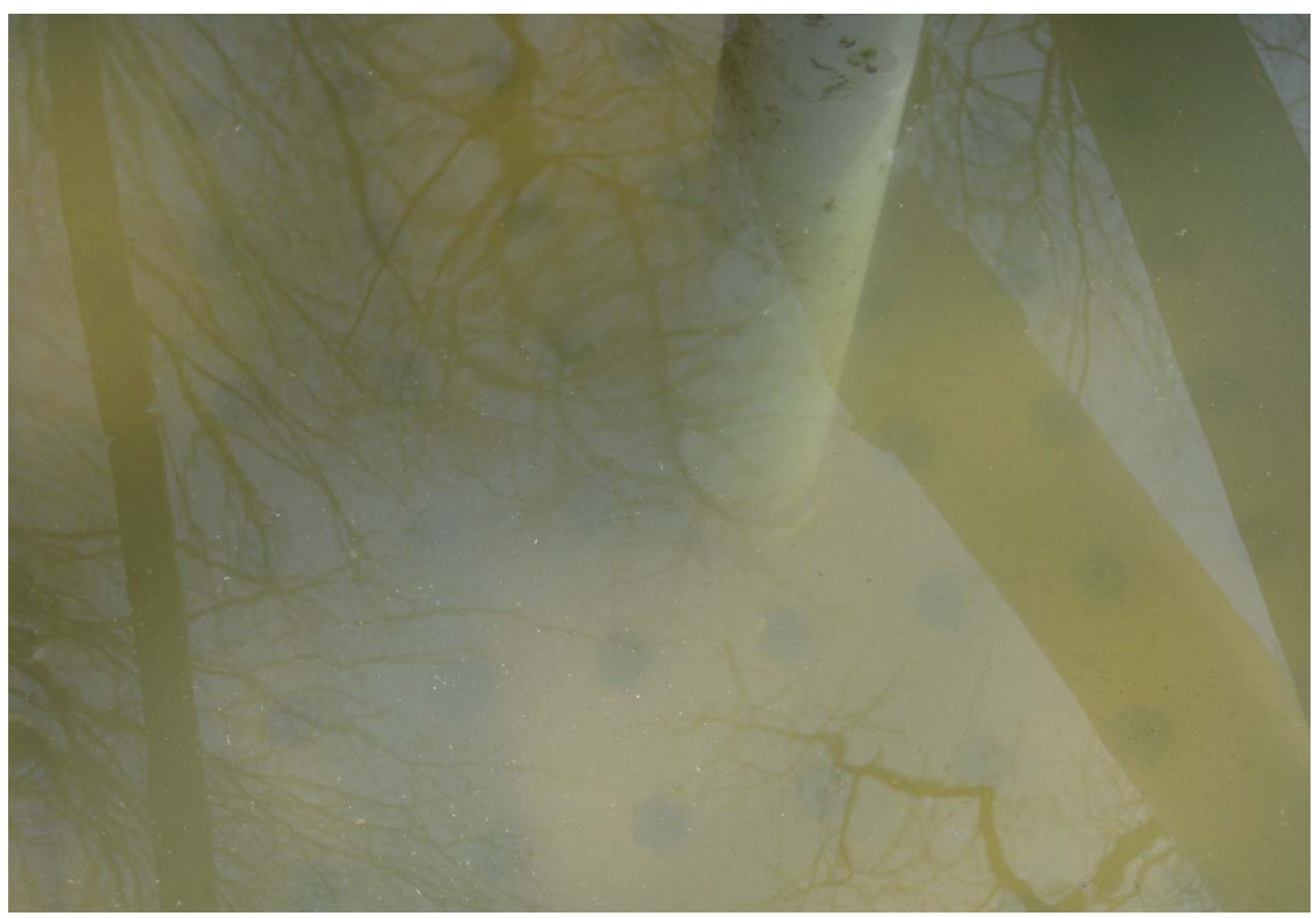

Figure 94: Siphon Test 2 Results 


\subsection{Cleaning Mechanism Conclusions and Suggestions}

From the previous tests of the two cleaning systems, the siphon system was more successful in removing solids from the bottom of the quiescent zone when compared to the sloped false bottom. The siphon system results were not successful enough to replace the manual method that is used at the present time.

Using Shields curve to find the critical shear stress of a non-cohesive solid particle is not a recommended method for measuring solids within an aquaculture raceway system. The solids within the raceway have cohesive properties, which Shields curve does not account for. The cohesive properties of the particles may be causing an undesirably large amount of friction. This cannot be ignored when determining the critical shear stress required to move settled particles on a smooth surface near the bottom of the quiescent zone.

It is suggested that the siphon system be used to drive the moving suction cleaner (13.1.3). Since the hydraulic energy used in the previous designs is not great enough to clean the entire bottom at once, it should instead be used to clean sections of the bottom over a period of time. This is recommended in order to effectively utilize the power of the siphon system. It is also suggested to use electric powered actuators and a timing system to activate and deactivate the siphon system. The previous suggestion will remove the need for the buoyancy and flap valve systems and allow for consistent cycle times. Actuators do not require much electricity therefore the power may be supplied via portable battery. This battery can be charged using a solar cell. 


\section{Acknowledgements}

I dedicate this thesis to the memory of my brother, Jason, a hard working and inspiring engineer. Jason opened my eyes to the engineering world. For this I give many thanks and much love to my brother.

I would like to thank Dr. Donald D. Gray for all of his advice and support throughout my years as a graduate student. I would also like to thank the rest of my committee members, Dr. John M. Kuhlman, Dr. Gary J. Morris, Dr. Wade W. Huebsch, and Dr. Kenneth Semmens for their support. Many thanks to my family, Wanda, David, and Brittany are deserved for their support and encouragement.

This work is supported by the Cooperative State Research Education and Extension Service, U.S. Department of Agriculture. The opinions, findings, conclusions, or recommendations expressed in this presentation are those of the author and do not necessarily reflect the view of the U.S. Department of Agriculture. 


\section{References}

“Automatic Pool Cleaner.” No Date. Lil Shark Pool Cleaner Image. 1800Pools.com. 20 April $2009<\mathrm{http}: / /$ www.1800pools.com/index/page/product/product_id/284/product_name/NE430++ Lil+Shark+Pool+Cleaner+Suction+Side $>$

Boersen, Gary; Westers, Harry, 1986. Waste Solids Control in Hatchery Raceways. The Progressive Fish Culturist, 48: 151-154.

Bradshaw, P., 1971. An Introduction to Turbulence and its Measurement. Pergamon Press, Oxford.

Frisch, Uriel, 1995. Turbulence. Cambridge University Press, New York, NY.

Garcia, Carolos Marcelo; Cantero, Mariano I.; Nino, Yarko; Garcia, Marcelo H., 2004 Acoustic Doppler Velocimeters (ADV) Performance Curves (APCs) sampling the flow turbulence. Ven Te Chow Hydrosystems Laboratory, Dept. Of Civil and Environmental Eng., Univ. of Illinois at Urbana-Champaign, IL, Dept. of Civil Eng., Univ. of Chile, Chile.

Grass, A.J., 1971. Structural features of turbulent flows over smooth and rough boundaries. J. Fluid Mech, 50, Part 2: 233-255.

Gray, Dr. Donald D., 1982. Class Notes. An Introduction to the Fluid Dynamics of Environmental Flows. Ch. 7, Turbulent Flows.

Gray, Dr. Donald D., 2008. Class Lecture. Hydrodynamic Design of Aquaculture Raceways. West Virginia University, Morgantown, WV. 12 February 2008.

Huggins, Dania, 2003. Analysis of sediment transport modeling using computational fluid dynamics (CFD). MS Thesis. Univ. Of Calif., Davis, CA.

Huggins, Dania, 2004. Analysis of sediment transport modeling using computational fluid dynamics (CFD). Aquacultural Eng., Univ. Of Calif., Davis, CA.

Juhani, Vesa N.; Mann, Jason J.; Sneddon, Scott. Method for Improving the Sinkability of Fish Food Pellets. Patent US 6174552. 2001.

Kraus, Nickolas C.; Lohrmann, Atle; Cabreram Ramon,1994. New Acoustic Mete for Measuring 3 D Laboratory Flows. Journal of Hydraulic Engineering, 120, No. 5: 406-412

Lohrmann, Atle; Cabrera, Ramon; Gelfenbaum, Guy; Haines, John, 1995. Direct Measurements of Reynolds Stress with an Acoustic Doppler Velocimeter. IEEE: 205-210.

Miller, Daniel. 2008. Using Aquaculture as a Post-mining Land Use in West Virginia. Mine Water Environment, 27: 122-126 
Nakagawa, H; Nezu, Iehisal; Tominga, A., 1983 Secondary Currents in a Straight Channel Flow and the Relation to its Aspect Ratio. Fourth Symposium on Turbulent Shear Flows, Karlsruhe, F.R. Germany: 3.8-3.13

Nezu, Iehisal; Rodi, Wolfgang, 1986. Open-Channel Flow Measurements With A Laser Doppler Anemometer. Journal of Hydraulic Engineering, 112, No. 5: 335-355.

Nikuradse, J; Reichardt H., 1945. Heat Transfer Through Turbulent Friction Layers. NACA TM 1047

Peterson, Eric L., 1999. Benthic shear stress and sediment condition. School of Eng. and the Cooperative Research Centre for Aquaculture, James Cook University, Townsville, Australia, Aquaculture Engineering, 21: 86-111.

Rumberg, Andrea T., 2004. Enhanced Solids Design Based on Characterization of Quiescent Zones Hydrodynamics in Flow-Through Aquaculture Systems . MS Thesis. West Virginia University., Morgantown, WV.

Schlichting, Herrmann, 1979. Boundary Layer Theory, Seventh ed. McGraw-Hill, New York, NY.

SonTek ADVField Acoustic Doppler Velocimeter Technical Documentation, 2001. San Diego, CA.

Stagle, Eric, 2006. Trout Thrive in Treated Mine Water: Feeding Habits Focus on New WVU Study. The Dominion Post, 13 February

Trowbridge, John H.; Geyer, W. Rockwell; Butman, Cheryl Ann; Chapman, Robert J, 1989. The 17-Meter Flume at the Coastal Research Laboratory. Part II Flow Characteristics. Woods Hole Oceanog. Inst.Tech. Rept.

Viadero, Roger C., Jr.; Rumberg, Andrea; Gray, Donald D.; Tierney, Aislinn E.; Semmens, Kenneth J., 2005. Acoustic Doppler velocimetry in aquaculture research: Raceway and quiescent zone hydrodynamics. Aquaculture Engineering, 34: 16-25.

Voulgaris, G.; Trowbridge, John H., 1997. Evaluation of the Acoustic Doppler Velocimeter (ADV) for Turbulence Measurements. Journal of Atmosphere and Oceanic Technology, 15: 272289

White, Frank M., 2006. Viscous Fluid Flow, Third ed. McGraw-Hill, New York, NY.

Young, Donald F.; Munson, Bruce R; Okiishi, Theodore H, 2004. A Brief Introduction to Fluid Mechanics.

Yuan, S.W., 1967. Foundations of Fluid Mechanics. Prentice-Hall, Inc., Englewood Cliffs, NJ. 


\section{Appendix A: Tables of Measured Values}

This appendix includes values of flow rates, water temperatures, mean velocities, RMS velocity, Reynolds stress, turbulence intensity, mean correlation (average correlation between all velocity components), mean SNR, and standard deviation.

Table 10: Velocity Measurements Taken on August 30, 2008

\begin{tabular}{|c|c|c|c|c|}
\hline Date Measured & $8 / 30 / 2008$ & & Flow Rate(Gallons/s) & Flow Rate $\left(\mathrm{cm}^{\wedge} 3 / \mathrm{s}\right)$ \\
\hline Water Temperature & $25.2^{\circ} \mathrm{C}$ & & \begin{tabular}{|l|}
4.2 \\
\end{tabular} & 15899 \\
\hline Raceway & A4 & & 4.2 & 15717 \\
\hline Sample Time & 6 Minutes & & 4.3 & 16194 \\
\hline Sample Rate & $10 \mathrm{~Hz}$ & Average & 4.2 & 15937 \\
\hline Screen Size & Large & & & \\
\hline QZ Length & $180 \mathrm{~cm}$ & & & \\
\hline QZ Width & $89 \mathrm{~cm}$ & & & \\
\hline QZ Water Depth & $89 \mathrm{~cm}$ & & & \\
\hline Measured Dist. from Wall & $45 \mathrm{~cm}$ & & & \\
\hline Measured Dist. from Start of QZ & $90 \mathrm{~cm}$ & & & \\
\hline Measured with & No Fish & & & \\
\hline
\end{tabular}


Table 11: Mean Velocity Values Measured on August 30, 2008

\begin{tabular}{|c|c|c|c|c|}
\hline Measurement \# & Meas. Distance From Bottom & Mean u & Mean v & Mean w \\
\hline & cm & $\mathrm{cm} / \mathrm{s}$ & $\mathrm{cm} / \mathrm{s}$ & $\mathrm{cm} / \mathrm{s}$ \\
\hline 1 & 5.16 & 1.05 & 0.31 & -0.14 \\
\hline 2 & 7.70 & 1.61 & 0.28 & -0.11 \\
\hline 3 & 10.24 & 1.99 & 0.04 & -0.17 \\
\hline 4 & 12.78 & 2.05 & 0.00 & -0.18 \\
\hline 5 & 15.32 & 2.31 & 0.07 & -0.13 \\
\hline 6 & 17.86 & 2.36 & 0.09 & -0.23 \\
\hline 7 & 20.40 & 2.40 & 0.13 & -0.18 \\
\hline 8 & 22.94 & 2.49 & 0.14 & -0.16 \\
\hline 9 & 25.48 & 2.52 & 0.17 & 0.05 \\
\hline 10 & 28.02 & 2.45 & 0.09 & 0.02 \\
\hline 11 & 30.56 & 2.45 & 0.06 & -0.02 \\
\hline 12 & 33.10 & 2.33 & 0.04 & 0.01 \\
\hline 13 & 35.64 & 2.45 & 0.22 & 0.11 \\
\hline 14 & 38.18 & 2.47 & 0.22 & 0.10 \\
\hline 15 & 40.72 & 2.48 & 0.17 & 0.09 \\
\hline 16 & 43.26 & 2.46 & 0.21 & 0.13 \\
\hline 17 & 45.80 & 2.51 & 0.16 & 0.02 \\
\hline 18 & 48.34 & 2.49 & 0.19 & 0.09 \\
\hline 19 & 50.88 & 2.50 & 0.24 & -0.01 \\
\hline 20 & 53.42 & 2.60 & 0.38 & -0.05 \\
\hline 21 & 55.96 & 2.63 & 0.26 & -0.02 \\
\hline 22 & 58.50 & 2.66 & 0.28 & 0.04 \\
\hline 23 & 61.04 & 2.69 & 0.31 & 0.05 \\
\hline 24 & 63.58 & 2.65 & 0.18 & -0.04 \\
\hline 25 & 66.12 & 2.83 & -0.07 & 0.01 \\
\hline
\end{tabular}


Table 12: Standard Deviation and SNR Measured on August 30, 2008

\begin{tabular}{|c|c|c|c|c|c|c|c|}
\hline Measurement \# & Stnd Dev u & Stnd Dev v & Stnd Dev w & Mean SNR u & Mean SNR v & Mean SNR w & Mean Correlation \\
\hline & $\mathrm{cm} / \mathrm{s}$ & $\mathrm{cm} / \mathrm{s}$ & $\mathrm{cm} / \mathrm{s}$ & & & & $\%$ \\
\hline 1 & 0.41 & 0.32 & 0.15 & 6.39 & 6.25 & 6.73 & 96.83 \\
\hline 2 & 0.49 & 0.38 & 0.30 & 7.43 & 7.32 & 7.79 & 95.62 \\
\hline 3 & 0.45 & 0.35 & 0.27 & 9.49 & 9.36 & 9.84 & 95.26 \\
\hline 4 & 0.52 & 0.37 & 0.34 & 10.33 & 10.15 & 10.75 & 94.97 \\
\hline 5 & 0.39 & 0.37 & 0.32 & 7.06 & 6.93 & 7.40 & 94.91 \\
\hline 6 & 0.39 & 0.38 & 0.29 & 6.00 & 5.79 & 6.34 & 95.21 \\
\hline 7 & 0.40 & 0.37 & 0.31 & 2.58 & 2.43 & 2.92 & 95.01 \\
\hline 8 & 0.35 & 0.29 & 0.26 & 5.53 & 5.30 & 5.86 & 94.52 \\
\hline 9 & 0.34 & 0.32 & 0.24 & -13.60 & 2.96 & -12.86 & 94.25 \\
\hline 10 & 0.32 & 0.29 & 0.24 & 17.03 & 17.08 & 17.37 & 94.83 \\
\hline 11 & 0.34 & 0.30 & 0.25 & 9.14 & 9.95 & 9.00 & 93.49 \\
\hline 12 & 0.29 & 0.28 & 0.24 & -2.69 & 13.57 & -2.38 & 93.36 \\
\hline 13 & 0.29 & 0.28 & 0.22 & 9.14 & 10.02 & 8.47 & 93.39 \\
\hline 14 & 0.29 & 0.25 & 0.20 & 9.58 & 10.51 & 8.97 & 93.68 \\
\hline 15 & 0.29 & 0.29 & 0.22 & 9.30 & 10.28 & 8.73 & 93.53 \\
\hline 16 & 0.30 & 0.24 & 0.21 & 9.42 & 10.37 & 8.74 & 93.49 \\
\hline 17 & 0.29 & 0.29 & 0.24 & 8.93 & 10.17 & 8.69 & 93.25 \\
\hline 18 & 0.29 & 0.28 & 0.24 & 8.82 & 9.97 & 8.58 & 92.67 \\
\hline 19 & 0.32 & 0.35 & 0.26 & 7.96 & 9.16 & 7.83 & 92.08 \\
\hline 20 & 0.30 & 0.29 & 0.24 & 9.13 & 10.44 & 8.89 & 92.73 \\
\hline 21 & 0.30 & 0.33 & 0.23 & 8.77 & 9.95 & 8.52 & 92.31 \\
\hline 22 & 0.34 & 0.32 & 0.27 & 6.94 & 8.01 & 6.72 & 88.48 \\
\hline 23 & 0.34 & 0.31 & 0.26 & 8.02 & 9.04 & 7.76 & 87.90 \\
\hline 24 & 0.36 & 0.35 & 0.30 & 7.18 & 8.22 & 6.90 & 87.53 \\
\hline 25 & 0.36 & 0.36 & 0.27 & 6.89 & 7.96 & 6.49 & 88.50 \\
\hline
\end{tabular}


Table 13: Reynolds Stress, TI, and RMS Measured on August 30, 3009

\begin{tabular}{|c|c|c|c|c|c|c|c|c|c|c|}
\hline Measurement \# & RS_uw & RS_vu & RS_wv & RS_uu & RS_vv & RS_ww & Urms & Vrms & Wrms & TI \\
\hline & $\mathrm{Pa}$ & $\mathrm{Pa}$ & $\mathrm{Pa}$ & $\mathrm{Pa}$ & $\mathrm{Pa}$ & $\mathrm{Pa}$ & $\mathrm{cm} / \mathrm{s}$ & $\mathrm{cm} / \mathrm{s}$ & $\mathrm{cm} / \mathrm{s}$ & \\
\hline 1 & 4.02E-03 & $-2.00 \mathrm{E}-03$ & $-3.31 E-04$ & $-1.71 \mathrm{E}-02$ & $-9.91 \mathrm{E}-03$ & $-2.27 \mathrm{E}-03$ & 4.15E-01 & 3.15E-01 & $1.51 \mathrm{E}-01$ & 2.84E-01 \\
\hline 2 & $7.16 \mathrm{E}-03$ & $-2.23 \mathrm{E}-03$ & $1.16 \mathrm{E}-03$ & $-2.41 \mathrm{E}-02$ & $-1.43 \mathrm{E}-02$ & $-9.20 \mathrm{E}-03$ & $4.92 \mathrm{E}-01$ & $3.79 \mathrm{E}-01$ & $3.04 \mathrm{E}-01$ & 2.43E-01 \\
\hline 3 & 4.24E-03 & $3.28 \mathrm{E}-03$ & $-4.75 E-04$ & $-2.03 E-02$ & $-1.24 \mathrm{E}-02$ & $-7.04 \mathrm{E}-03$ & $4.52 \mathrm{E}-01$ & 3.53E-01 & $2.66 \mathrm{E}-01$ & $1.83 \mathrm{E}-01$ \\
\hline 4 & 8.60E-03 & $3.28 \mathrm{E}-03$ & $-7.25 E-04$ & $-2.66 \mathrm{E}-02$ & $-1.39 \mathrm{E}-02$ & $-1.13 \mathrm{E}-02$ & $5.16 \mathrm{E}-01$ & $3.74 \mathrm{E}-01$ & 3.37E-01 & 2.02E-01 \\
\hline 5 & $3.85 \mathrm{E}-03$ & $1.99 \mathrm{E}-03$ & $6.42 \mathrm{E}-04$ & $-1.50 \mathrm{E}-02$ & $-1.37 \mathrm{E}-02$ & $-1.05 E-02$ & $3.88 \mathrm{E}-01$ & 3.71E-01 & $3.25 \mathrm{E}-01$ & 1.57E-01 \\
\hline 6 & 3.23E-03 & $2.44 \mathrm{E}-03$ & $-3.38 \mathrm{E}-04$ & $-1.53 \mathrm{E}-02$ & $-1.43 \mathrm{E}-02$ & $-8.45 E-03$ & $3.92 \mathrm{E}-01$ & $3.78 \mathrm{E}-01$ & $2.91 \mathrm{E}-01$ & $1.51 \mathrm{E}-01$ \\
\hline 7 & 3.09E-03 & $2.15 \mathrm{E}-03$ & $-8.90 \mathrm{E}-04$ & $-1.60 \mathrm{E}-02$ & $-1.34 \mathrm{E}-02$ & $-9.88 \mathrm{E}-03$ & $4.00 \mathrm{E}-01$ & 3.67E-01 & $3.15 \mathrm{E}-01$ & $1.50 \mathrm{E}-01$ \\
\hline 8 & $7.12 \mathrm{E}-04$ & $-1.88 \mathrm{E}-04$ & $-2.09 E-04$ & $-1.21 \mathrm{E}-02$ & $-8.22 \mathrm{E}-03$ & $-6.78 \mathrm{E}-03$ & 3.49E-01 & 2.87E-01 & $2.61 \mathrm{E}-01$ & $1.21 \mathrm{E}-01$ \\
\hline 9 & $4.30 \mathrm{E}-04$ & $2.07 \mathrm{E}-03$ & $-1.83 \mathrm{E}-04$ & $-1.17 \mathrm{E}-02$ & $-1.05 E-02$ & $-5.69 \mathrm{E}-03$ & 3.43E-01 & $3.24 \mathrm{E}-01$ & $2.39 \mathrm{E}-01$ & $1.21 \mathrm{E}-01$ \\
\hline 10 & $-2.07 \mathrm{E}-03$ & $-4.70 \mathrm{E}-04$ & $5.42 \mathrm{E}-04$ & $-1.04 \mathrm{E}-02$ & $-8.21 \mathrm{E}-03$ & $-5.88 \mathrm{E}-03$ & 3.23E-01 & 2.87E-01 & $2.43 \mathrm{E}-01$ & 1.17E-01 \\
\hline 11 & $-1.50 \mathrm{E}-03$ & $-8.32 E-04$ & $-5.25 E-04$ & $-1.14 \mathrm{E}-02$ & $-8.98 \mathrm{E}-03$ & $-6.21 E-03$ & $3.38 \mathrm{E}-01$ & $3.00 \mathrm{E}-01$ & $2.50 \mathrm{E}-01$ & $1.22 \mathrm{E}-01$ \\
\hline 12 & $-1.02 \mathrm{E}-03$ & 3.29E-04 & $-2.77 \mathrm{E}-05$ & $-8.32 \mathrm{E}-03$ & $-8.01 \mathrm{E}-03$ & $-5.79 E-03$ & $2.89 \mathrm{E}-01$ & $2.84 \mathrm{E}-01$ & $2.41 \mathrm{E}-01$ & 1.17E-01 \\
\hline 13 & $1.53 \mathrm{E}-04$ & $-7.38 \mathrm{E}-04$ & $1.06 \mathrm{E}-03$ & $-8.54 \mathrm{E}-03$ & $-7.93 \mathrm{E}-03$ & $-4.66 \mathrm{E}-03$ & 2.93E-01 & $2.82 \mathrm{E}-01$ & $2.16 \mathrm{E}-01$ & $1.08 \mathrm{E}-01$ \\
\hline 14 & $-9.53 E-04$ & $-2.14 \mathrm{E}-04$ & $6.63 \mathrm{E}-04$ & $-8.55 E-03$ & $-6.22 \mathrm{E}-03$ & $-4.10 \mathrm{E}-03$ & 2.93E-01 & $2.50 \mathrm{E}-01$ & $2.03 \mathrm{E}-01$ & $1.01 \mathrm{E}-01$ \\
\hline 15 & 6.67E-04 & $-1.52 \mathrm{E}-03$ & $-8.53 \mathrm{E}-04$ & $-8.53 E-03$ & $-8.28 \mathrm{E}-03$ & $-4.91 E-03$ & 2.93E-01 & $2.88 \mathrm{E}-01$ & $2.22 \mathrm{E}-01$ & 1.09E-01 \\
\hline 16 & $-1.19 E-04$ & $-1.26 \mathrm{E}-03$ & $-5.93 E-04$ & $-8.80 \mathrm{E}-03$ & $-5.84 \mathrm{E}-03$ & $-4.34 \mathrm{E}-03$ & 2.97E-01 & 2.42E-01 & $2.09 \mathrm{E}-01$ & $1.02 \mathrm{E}-01$ \\
\hline 17 & $-6.91 E-04$ & $-1.38 \mathrm{E}-03$ & $-1.16 \mathrm{E}-03$ & $-8.32 \mathrm{E}-03$ & $-8.28 \mathrm{E}-03$ & $-5.94 \mathrm{E}-03$ & $2.89 \mathrm{E}-01$ & $2.88 \mathrm{E}-01$ & $2.44 \mathrm{E}-01$ & 1.09E-01 \\
\hline 18 & $-2.22 \mathrm{E}-04$ & $-1.10 \mathrm{E}-03$ & $3.82 \mathrm{E}-04$ & $-8.56 \mathrm{E}-03$ & $-8.09 E-03$ & $-5.53 \mathrm{E}-03$ & 2.93E-01 & $2.85 \mathrm{E}-01$ & $2.36 \mathrm{E}-01$ & $1.09 \mathrm{E}-01$ \\
\hline 19 & $1.65 \mathrm{E}-04$ & $-7.04 \mathrm{E}-04$ & $-6.64 \mathrm{E}-04$ & $-9.93 E-03$ & $-1.25 \mathrm{E}-02$ & $-6.54 \mathrm{E}-03$ & $3.16 \mathrm{E}-01$ & 3.54E-01 & $2.56 \mathrm{E}-01$ & 1.24E-01 \\
\hline 20 & $-7.32 E-05$ & $-9.21 \mathrm{E}-04$ & $9.85 \mathrm{E}-04$ & $-9.20 \mathrm{E}-03$ & $-8.53 E-03$ & $-5.80 \mathrm{E}-03$ & $3.04 \mathrm{E}-01$ & $2.93 \mathrm{E}-01$ & $2.41 \mathrm{E}-01$ & 1.07E-01 \\
\hline 21 & $9.88 \mathrm{E}-04$ & $-1.10 \mathrm{E}-03$ & $-3.50 \mathrm{E}-04$ & $-9.20 \mathrm{E}-03$ & $-1.06 \mathrm{E}-02$ & $-5.41 \mathrm{E}-03$ & $3.04 \mathrm{E}-01$ & $3.26 \mathrm{E}-01$ & $2.33 \mathrm{E}-01$ & $1.10 \mathrm{E}-01$ \\
\hline 22 & $5.88 \mathrm{E}-04$ & $-1.31 \mathrm{E}-03$ & $-3.76 E-04$ & $-1.17 \mathrm{E}-02$ & $-1.01 \mathrm{E}-02$ & $-7.27 \mathrm{E}-03$ & $3.42 \mathrm{E}-01$ & $3.19 \mathrm{E}-01$ & $2.70 \mathrm{E}-01$ & 1.17E-01 \\
\hline 23 & $1.02 \mathrm{E}-03$ & $-1.91 \mathrm{E}-03$ & $-2.89 E-04$ & $-1.14 \mathrm{E}-02$ & $-9.80 \mathrm{E}-03$ & $-6.91 E-03$ & 3.38E-01 & $3.14 \mathrm{E}-01$ & 2.63E-01 & 1.13E-01 \\
\hline 24 & $9.76 \mathrm{E}-04$ & $-1.35 E-03$ & $-5.79 E-04$ & $-1.29 \mathrm{E}-02$ & $-1.22 \mathrm{E}-02$ & $-8.74 \mathrm{E}-03$ & 3.59E-01 & $3.49 \mathrm{E}-01$ & $2.96 \mathrm{E}-01$ & 1.26E-01 \\
\hline 25 & $6.15 \mathrm{E}-04$ & $-1.05 E-03$ & $-8.81 \mathrm{E}-04$ & $-1.27 \mathrm{E}-02$ & $-1.27 \mathrm{E}-02$ & $-7.35 E-03$ & 3.57E-01 & 3.57E-01 & $2.72 \mathrm{E}-01$ & 1.17E-01 \\
\hline
\end{tabular}


Table 14: Velocity Measurements Taken on September 4, 2008

\begin{tabular}{|c|c|c|c|c|}
\hline Date Measured & $9 / 4 / 2008$ & & Flow Rate(Gallons/s) & Flow Rate(cm^3/s) \\
\hline Water Temperature & $22.2^{\circ} \mathrm{C}$ & & 4.0 & 15089 \\
\hline Raceway & $\mathrm{A} 4$ & & 4.4 & 16497 \\
\hline Sample Time & $6 \mathrm{Minutes}$ & & 4.2 & 15921 \\
\hline Sample Rate & $10 \mathrm{~Hz}$ & Average & 4.2 & 15836 \\
\hline Screen Size & Large & & & \\
\hline QZ Length & $180 \mathrm{~cm}$ & & & \\
\hline QZ Width & $89 \mathrm{~cm}$ & & & \\
\hline QZ Water Depth & $89 \mathrm{~cm}$ & & & \\
\hline Measured Dist. from Wall & $45 \mathrm{~cm}$ & & & \\
\hline Measured Dist. from Start of QZ & $90 \mathrm{~cm}$ & & & \\
\hline Measured with & No fish & & & \\
\hline
\end{tabular}


Table 15: Mean Velocity Values Measured on September 4, 2008

\begin{tabular}{|c|c|c|c|c|}
\hline Measurement \# & Meas. Distance From Bottom & Mean u & Mean v & Mean w \\
\hline & $\mathrm{cm}$ & $\mathrm{cm} / \mathrm{s}$ & $\mathrm{cm} / \mathrm{s}$ & $\mathrm{cm} / \mathrm{s}$ \\
\hline 26 & 5.16 & 1.12 & 0.06 & 0.00 \\
\hline 27 & 7.70 & 1.34 & 0.16 & -0.03 \\
\hline 28 & 10.24 & 1.57 & -0.02 & -0.01 \\
\hline 29 & 12.78 & 1.87 & -0.01 & -0.11 \\
\hline 30 & 15.32 & 2.12 & -0.03 & -0.09 \\
\hline 31 & 17.86 & 2.05 & -0.04 & 0.01 \\
\hline 32 & 20.40 & 2.33 & 0.09 & -0.04 \\
\hline 33 & 22.94 & 2.41 & 0.05 & -0.04 \\
\hline 34 & 25.48 & 2.43 & 0.00 & 0.11 \\
\hline 35 & 28.02 & 2.43 & 0.13 & 0.01 \\
\hline 36 & 30.56 & 2.43 & 0.02 & 0.03 \\
\hline 37 & 33.10 & 2.41 & 0.13 & 0.03 \\
\hline 38 & 35.64 & 2.46 & 0.09 & 0.06 \\
\hline 39 & 38.18 & 2.48 & 0.02 & 0.06 \\
\hline 40 & 40.72 & 2.44 & 0.02 & -0.02 \\
\hline 41 & 43.26 & 2.42 & 0.10 & 0.07 \\
\hline 42 & 45.80 & 2.49 & 0.04 & 0.06 \\
\hline 43 & 48.34 & 2.48 & 0.02 & 0.01 \\
\hline 44 & 50.88 & 2.56 & -0.01 & 0.02 \\
\hline 45 & 53.42 & 2.49 & -0.02 & -0.02 \\
\hline 46 & 55.96 & 2.65 & 0.01 & -0.04 \\
\hline 47 & 58.50 & 2.69 & -0.01 & -0.02 \\
\hline 48 & 61.04 & 2.69 & 0.08 & -0.03 \\
\hline 49 & 63.58 & 2.75 & 0.05 & -0.03 \\
\hline 50 & 66.12 & 2.72 & -0.22 & 0.11 \\
\hline 51 & 68.66 & 2.76 & -0.10 & -0.04 \\
\hline 52 & 71.20 & 2.74 & -0.13 & 0.07 \\
\hline 53 & 73.74 & 2.70 & -0.11 & 0.05 \\
\hline 54 & 76.28 & 2.71 & -0.18 & 0.04 \\
\hline 55 & 78.82 & 2.70 & -0.12 & 0.06 \\
\hline
\end{tabular}


Table 16: Standard Deviation and SNR Measured on September 4, 2008

\begin{tabular}{|c|c|c|c|c|c|c|c|}
\hline Measurement \# & Stnd Dev u & Stnd Dev v & Stnd Dev w & Mean SNR u & Mean SNR v & Mean SNR w & Mean Correlation \\
\hline & $\mathrm{cm} / \mathrm{s}$ & $\mathrm{cm} / \mathrm{s}$ & $\mathrm{cm} / \mathrm{s}$ & \begin{tabular}{|c|}
$d B$ \\
\end{tabular} & $\mathrm{~dB}$ & $\mathrm{~dB}$ & $\%$ \\
\hline 26 & 0.42 & 0.33 & 0.22 & 11.10 & 11.52 & 10.60 & 95.51 \\
\hline 27 & 0.42 & 0.42 & 0.29 & 10.50 & 10.56 & 9.92 & 94.91 \\
\hline 28 & 0.47 & 0.39 & 0.35 & 10.54 & 10.59 & 9.82 & 94.74 \\
\hline 29 & 0.40 & 0.39 & 0.33 & 10.33 & 10.30 & 9.64 & 94.53 \\
\hline 30 & 0.40 & 0.37 & 0.26 & 10.18 & 10.21 & 9.54 & 94.48 \\
\hline 31 & 0.44 & 0.37 & 0.30 & 9.96 & 10.07 & 9.40 & 94.30 \\
\hline 32 & 0.36 & 0.32 & 0.26 & 9.77 & 9.79 & 9.15 & 94.03 \\
\hline 33 & 0.27 & 0.30 & 0.23 & 10.68 & 10.73 & 9.95 & 94.44 \\
\hline 34 & 0.27 & 0.28 & 0.21 & 10.35 & 10.31 & 9.58 & 94.18 \\
\hline 35 & 0.26 & 0.29 & 0.21 & 10.53 & 10.73 & 9.95 & 94.30 \\
\hline 36 & 0.26 & 0.29 & 0.18 & 10.11 & 10.16 & 9.51 & 94.08 \\
\hline 37 & 0.26 & 0.28 & 0.18 & 10.60 & 10.79 & 10.03 & 94.39 \\
\hline 38 & 0.25 & 0.27 & 0.17 & 10.66 & 10.77 & 10.02 & 94.34 \\
\hline 39 & 0.27 & 0.23 & 0.21 & 10.41 & 10.59 & 9.87 & 94.12 \\
\hline 40 & 0.25 & 0.27 & 0.19 & 10.91 & 11.01 & 10.21 & 94.31 \\
\hline 41 & 0.25 & 0.26 & 0.19 & 11.24 & 11.01 & 10.20 & 93.51 \\
\hline 42 & 0.24 & 0.25 & 0.18 & 9.82 & 10.15 & 9.87 & 93.64 \\
\hline 43 & 0.24 & 0.25 & 0.17 & 10.24 & 10.68 & 10.34 & 94.12 \\
\hline 44 & 0.28 & 0.26 & 0.22 & 10.74 & 11.18 & 10.83 & 94.22 \\
\hline 45 & 0.26 & 0.28 & 0.21 & 10.48 & 11.05 & 10.62 & 94.05 \\
\hline 46 & 0.27 & 0.27 & 0.22 & 10.94 & 11.41 & 11.02 & 94.04 \\
\hline 47 & 0.30 & 0.29 & 0.22 & 10.93 & 11.54 & 11.06 & 94.19 \\
\hline 48 & 0.27 & 0.32 & 0.25 & 10.70 & 11.30 & 10.79 & 94.02 \\
\hline 49 & 0.29 & 0.33 & 0.23 & 10.59 & 11.24 & 10.76 & 94.02 \\
\hline 50 & 0.30 & 0.27 & 0.27 & 11.48 & 11.84 & 10.92 & 93.84 \\
\hline 51 & 0.34 & 0.33 & 0.28 & 12.05 & 12.58 & 11.56 & 94.19 \\
\hline 52 & 0.34 & 0.33 & 0.28 & 11.80 & 12.38 & 11.43 & 94.07 \\
\hline 53 & 0.34 & 0.32 & 0.29 & 10.46 & 11.01 & 10.10 & 93.67 \\
\hline 54 & 0.39 & 0.32 & 0.34 & 11.20 & 11.86 & 11.02 & 93.87 \\
\hline 55 & 0.38 & 0.30 & 0.36 & 11.61 & 12.27 & 11.26 & 93.77 \\
\hline
\end{tabular}


Table 17: Reynolds Stress, TI, and RMS Measured on September 4, 2008

\begin{tabular}{|c|c|c|c|c|c|c|c|c|c|c|}
\hline Measurement \# & RS_uw & RS_vu & RS_wv & RS_uu & RS_vv & RS_ww & Urms & Vrms & Wrms & TI \\
\hline & $\mathrm{Pa}$ & $\mathrm{Pa}$ & $\mathrm{Pa}$ & $\mathrm{Pa}$ & $\mathrm{Pa}$ & $\mathrm{Pa}$ & $\mathrm{cm} / \mathrm{s}$ & $\mathrm{cm} / \mathrm{s}$ & $\mathrm{cm} / \mathrm{s}$ & \\
\hline 26 & 3.97E-03 & $8.54 \mathrm{E}-04$ & $-7.13 E-04$ & $-1.77 \mathrm{E}-02$ & $-1.10 \mathrm{E}-02$ & $-4.98 \mathrm{E}-03$ & $4.22 \mathrm{E}-01$ & 3.32E-01 & $2.24 \mathrm{E}-01$ & $3.00 \mathrm{E}-01$ \\
\hline 27 & $.02 \mathrm{E}-03$ & L.67E-03 & $1.62 \mathrm{E}-03$ & $-1.78 \mathrm{E}-02$ & $-1.77 \mathrm{E}-02$ & $-8.12 \mathrm{E}-03$ & $4.22 \mathrm{E}-01$ & $4.21 \mathrm{E}-01$ & $.86 \mathrm{E}-01$ & 2.82E-01 \\
\hline 28 & $7.72 \mathrm{E}-03$ & 2.30E-03 & 4.73E-04 & $-2.20 \mathrm{E}-02$ & $-1.53 \mathrm{E}-02$ & $-1.24 \mathrm{E}-02$ & 4.70E-01 & $3.92 \mathrm{E}-01$ & 3.52E-01 & $2.59 \mathrm{E}-01$ \\
\hline 29 & $.92 \mathrm{E}-03$ & $1.02 \mathrm{E}-04$ & $1.28 \mathrm{E}-03$ & $-1.61 \mathrm{E}-02$ & $-1.50 \mathrm{E}-02$ & $-1.11 \mathrm{E}-02$ & $4.02 \mathrm{E}-01$ & 3.88E-01 & 3.34E-01 & 2.00E-01 \\
\hline 30 & $3.56 \mathrm{E}-03$ & 1.69E-03 & $1.40 \mathrm{E}-03$ & $-1.62 \mathrm{E}-02$ & $-1.34 \mathrm{E}-02$ & $-6.56 \mathrm{E}-03$ & 4.03E-01 & $3.66 \mathrm{E}-01$ & 2.57E-01 & \\
\hline 31 & $44 \mathrm{E}-03$ & 3.49E-03 & $-6.47 \mathrm{E}-04$ & $-1.97 \mathrm{E}-02$ & $-1.40 \mathrm{E}-02$ & $-8.81 E-03$ & $4.45 \mathrm{E}-01$ & 3.75E-01 & 2.97E-01 & \\
\hline 32 & $.71 \mathrm{E}-03$ & $1.52 \mathrm{E}-03$ & $-6.30 \mathrm{E}-04$ & $-1.31 \mathrm{E}-02$ & $-1.04 \mathrm{E}-02$ & $-6.75 E-03$ & $3.63 \mathrm{E}-01$ & $3.24 \mathrm{E}-01$ & & \\
\hline 33 & 8.59E-04 & $1.03 \mathrm{E}-03$ & $-1.11 \mathrm{E}-03$ & $-7.12 \mathrm{E}-03$ & $-9.10 \mathrm{E}-03$ & $-5.20 \mathrm{E}-03$ & 2.67E-01 & $3.02 \mathrm{E}-01$ & $2.28 \mathrm{E}-01$ & \\
\hline 34 & 7.31E-05 & $-7.67 \mathrm{E}-05$ & $-2.14 \mathrm{E}-04$ & $-7.06 \mathrm{E}-03$ & $-7.73 \mathrm{E}-03$ & $-4.36 \mathrm{E}-03$ & $2.66 \mathrm{E}-01$ & 2.79E- 01 & $2.09 \mathrm{E}-01$ & \\
\hline 35 & $-1.13 E-04$ & $1.91 \mathrm{E}-05$ & $-3.43 E-04$ & $-6.57 \mathrm{E}-03$ & $-8.18 \mathrm{E}-03$ & $-4.56 \mathrm{E}-03$ & $2.57 \mathrm{E}-01$ & 2.87E-01 & $2.14 \mathrm{E}-01$ & \\
\hline 36 & $-1.84 \mathrm{E}-04$ & $-1.05 E-04$ & $6.60 \mathrm{E}-04$ & $-6.90 \mathrm{E}-03$ & $-8.14 \mathrm{E}-03$ & $-3.40 \mathrm{E}-03$ & 2.63E- 01 & $2.86 \mathrm{E}-01$ & $1.85 \mathrm{E}-01$ & \\
\hline 37 & $-6.36 \mathrm{E}-05$ & $1.19 \mathrm{E}-04$ & $6.68 \mathrm{E}-05$ & $-6.86 \mathrm{E}-03$ & $-7.56 \mathrm{E}-03$ & $-3.06 \mathrm{E}-03$ & 2.63E-01 & $2.75 \mathrm{E}-01$ & 1.75E-01 & $1.00 \mathrm{E}-01$ \\
\hline 38 & 1.79E-04 & $1.14 \mathrm{E}-03$ & $7.56 \mathrm{E}-04$ & $-6.37 \mathrm{E}-03$ & $-7.16 \mathrm{E}-03$ & $-2.90 \mathrm{E}-03$ & $2.53 \mathrm{E}-01$ & $2.68 \mathrm{E}-01$ & $1.71 \mathrm{E}-01$ & $9.50 \mathrm{E}-02$ \\
\hline 39 & $-2.14 \mathrm{E}-04$ & $2.36 \mathrm{E}-04$ & $-3.82 E-05$ & $-7.30 \mathrm{E}-03$ & $-5.38 \mathrm{E}-03$ & $-4.46 \mathrm{E}-03$ & 2.71E-01 & 2.32E-01 & $2.12 \mathrm{E}-01$ & $9.65 \mathrm{E}$ \\
\hline 40 & $-5.69 \mathrm{E}-04$ & $-9.18 \mathrm{E}-04$ & $-5.01 E-04$ & $-6.28 \mathrm{E}-03$ & $-7.42 \mathrm{E}-03$ & $-3.45 E-03$ & $2.51 \mathrm{E}-01$ & $2.73 \mathrm{E}-01$ & $1.86 \mathrm{E}-01$ & $9.83 \mathrm{E}-02$ \\
\hline 41 & $-4.01 \mathrm{E}-04$ & 3.80E-04 & $6.32 \mathrm{E}-04$ & $-6.12 \mathrm{E}-03$ & $-6.62 \mathrm{E}-03$ & $-3.72 E-03$ & $2.48 \mathrm{E}-01$ & $2.58 \mathrm{E}-01$ & 1.93E-01 & $9.67 \mathrm{E}-02$ \\
\hline 42 & $-2.28 \mathrm{E}-04$ & $3.58 \mathrm{E}-04$ & $7.75 \mathrm{E}-05$ & $-5.97 \mathrm{E}-03$ & $-5.98 \mathrm{E}-03$ & $-3.40 \mathrm{E}-03$ & $2.45 \mathrm{E}-01$ & $2.45 \mathrm{E}-01$ & $1.85 \mathrm{E}-01$ & $9.08 \mathrm{E}-02$ \\
\hline 43 & $-3.43 E-05$ & $-4.28 \mathrm{E}-04$ & $-1.31 E-04$ & $-5.72 \mathrm{E}-03$ & $-6.27 \mathrm{E}-03$ & $-2.78 \mathrm{E}-03$ & $2.40 \mathrm{E}-01$ & $2.51 \mathrm{E}-01$ & 1.67E-01 & 8.97E-02 \\
\hline 44 & 8.63E-04 & $-3.67 \mathrm{E}-04$ & 3.57E-04 & $-7.65 E-03$ & $-6.89 \mathrm{E}-03$ & $-4.66 \mathrm{E}-03$ & 2.77E- 01 & 2.63E-01 & $2.16 \mathrm{E}-01$ & $9.92 \mathrm{E}-02$ \\
\hline 45 & $-4.61 E-05$ & $2.26 \mathrm{E}-04$ & $-1.13 \mathrm{E}-03$ & $-6.95 E-03$ & $-7.81 \mathrm{E}-03$ & $-4.28 \mathrm{E}-03$ & $2.64 \mathrm{E}-01$ & $2.80 \mathrm{E}-01$ & 2.07E-01 & $1.01 \mathrm{E}-01$ \\
\hline 46 & 7.74E-05 & $-6.35 E-04$ & 4.41E-05 & $-7.29 \mathrm{E}-03$ & $-7.28 \mathrm{E}-03$ & $-4.68 \mathrm{E}-03$ & $2.70 \mathrm{E}-01$ & 2.70E-01 & 2.17E- 01 & $9.58 \mathrm{E}-02$ \\
\hline 47 & $1.00 \mathrm{E}-03$ & $-3.38 \mathrm{E}-04$ & $-5.80 \mathrm{E}-05$ & $-8.71 \mathrm{E}-03$ & $-8.18 \mathrm{E}-03$ & $-4.86 \mathrm{E}-03$ & $2.96 \mathrm{E}-01$ & 2.87E-01 & $2.21 \mathrm{E}-01$ & $1.00 \mathrm{E}-01$ \\
\hline 48 & $1.98 \mathrm{E}-04$ & $2.00 \mathrm{E}-04$ & $9.21 \mathrm{E}-04$ & $-7.53 \mathrm{E}-03$ & $-9.91 \mathrm{E}-03$ & $-6.11 E-03$ & $2.75 \mathrm{E}-01$ & $3.15 \mathrm{E}-01$ & $2.48 \mathrm{E}-01$ & $1.04 \mathrm{E}-01$ \\
\hline 49 & $-6.03 E-04$ & $-1.66 \mathrm{E}-04$ & $1.04 \mathrm{E}-04$ & $-8.45 E-03$ & $-1.06 \mathrm{E}-02$ & $-5.48 \mathrm{E}-03$ & $2.91 \mathrm{E}-01$ & 3.26E-01 & 2.34E-01 & $1.04 \mathrm{E}-01$ \\
\hline 50 & $-1.00 \mathrm{E}-03$ & $-6.46 \mathrm{E}-04$ & $-3.17 E-04$ & $-8.68 \mathrm{E}-03$ & $-7.30 \mathrm{E}-03$ & $-7.32 \mathrm{E}-03$ & $2.95 \mathrm{E}-01$ & 2.71E-01 & $2.71 \mathrm{E}-01$ & $1.02 \mathrm{E}-01$ \\
\hline 51 & $2.18 \mathrm{E}-04$ & $-1.26 \mathrm{E}-03$ & $1.45 \mathrm{E}-03$ & $-1.15 \mathrm{E}-02$ & $-1.11 \mathrm{E}-02$ & $-7.66 \mathrm{E}-03$ & $3.40 \mathrm{E}-01$ & 3.34E-01 & 2.77E-01 & 1.15E-01 \\
\hline 52 & $-4.33 E-05$ & $-1.47 \mathrm{E}-03$ & $6.02 \mathrm{E}-04$ & $-1.18 \mathrm{E}-02$ & $-1.08 \mathrm{E}-02$ & $-7.82 \mathrm{E}-03$ & $3.44 \mathrm{E}-01$ & 3.30E-01 & $2.80 \mathrm{E}-01$ & 1.16E-01 \\
\hline 53 & $-1.17 \mathrm{E}-04$ & $1.06 \mathrm{E}-04$ & $-4.42 E-04$ & $-1.17 \mathrm{E}-02$ & $-1.05 E-02$ & $-8.44 \mathrm{E}-03$ & $3.43 \mathrm{E}-01$ & 3.25E-01 & $2.91 \mathrm{E}-01$ & 1.19E-01 \\
\hline 54 & $-6.41 E-04$ & 1.37E-04 & $4.16 \mathrm{E}-04$ & $-1.50 \mathrm{E}-02$ & $-1.03 \mathrm{E}-02$ & $-1.14 \mathrm{E}-02$ & 3.87E-01 & $3.22 \mathrm{E}-01$ & $3.38 \mathrm{E}-01$ & $1.29 \mathrm{E}-01$ \\
\hline 55 & $3.30 \mathrm{E}-04$ & $-6.39 E-04$ & 7.24E-04 & $-1.41 \mathrm{E}-02$ & $-9.08 \mathrm{E}-03$ & $-1.28 \mathrm{E}-02$ & $3.76 \mathrm{E}-01$ & $3.02 \mathrm{E}-01$ & $3.58 \mathrm{E}-01$ & $1.28 \mathrm{E}-01$ \\
\hline
\end{tabular}


Table 18: Velocity Measurements Taken on September 30, 2008

\begin{tabular}{|c|c|c|c|c|}
\hline Date Measured & $9 / 30 / 2008$ & & Flow Rate(Gallons/s) & Flow Rate(cm^3/s) \\
\hline Water Temperature & $21.8^{\circ} \mathrm{C}$ & & No Data & No Data \\
\hline Raceway & A2 & & No Data & No Data \\
\hline Sample Time & 6 Minutes & & No Data & No Data \\
\hline Sample Rate & $10 \mathrm{~Hz}$ & Average & No Data & No Data \\
\hline Screen Size & Small $($ Dirty) & & & \\
\hline QZ Length & $180 \mathrm{~cm}$ & & & \\
\hline QZ Width & $91 \mathrm{~cm}$ & & & \\
\hline QZ Water Depth & $89 \mathrm{~cm}$ & & & \\
\hline Measured Dist. from Wall & $45 \mathrm{~cm}$ & & & \\
\hline Measured Dist. from Start of QZ & 90 & & & \\
\hline Measured with & $\approx 5000$ Striped Bass & & & \\
\hline
\end{tabular}

Table 19: Mean Velocity Values Measured on September 30, 2008

\begin{tabular}{|c|c|c|c|c|}
\hline Measurement \# & Meas. Distance From Bottom & Mean u & Mean v & Mean w \\
\hline & $\mathbf{c m}$ & $\mathrm{cm} / \mathrm{s}$ & $\mathrm{cm} / \mathrm{s}$ & $\mathrm{cm} / \mathbf{s}$ \\
\hline 56 & 5.16 & 1.94 & -0.03 & 0.06 \\
\hline 57 & 7.70 & 1.93 & 0.02 & 0.01 \\
\hline 58 & 10.24 & 1.85 & 0.07 & 0.04 \\
\hline 59 & 12.78 & 1.75 & 0.05 & 0.04 \\
\hline 60 & 15.32 & 1.88 & 0.01 & 0.08 \\
\hline 61 & 17.86 & 2.00 & 0.06 & 0.15 \\
\hline 62 & 20.40 & 1.95 & 0.06 & 0.20 \\
\hline 63 & 22.94 & 1.83 & 0.00 & 0.22 \\
\hline 64 & 25.48 & 1.53 & -0.08 & 0.19 \\
\hline 65 & 28.02 & 1.64 & -0.13 & 0.29 \\
\hline 66 & 30.56 & 1.57 & -0.14 & 0.33 \\
\hline 67 & 33.10 & 1.46 & -0.09 & 0.32 \\
\hline 68 & 35.64 & 1.46 & -0.05 & 0.34 \\
\hline 69 & 38.18 & 1.31 & 0.01 & 0.27 \\
\hline 70 & 40.72 & 1.25 & 0.08 & 0.22 \\
\hline 71 & 43.26 & 1.36 & 0.07 & 0.09 \\
\hline 72 & 45.80 & 1.63 & -0.01 & 0.10 \\
\hline 73 & 48.34 & 1.84 & 0.01 & 0.08 \\
\hline 74 & 50.88 & 2.22 & 0.01 & 0.09 \\
\hline 75 & 53.42 & 2.32 & 0.03 & 0.17 \\
\hline
\end{tabular}


Table 20: Standard Deviation and SNR Measured on September 30, 2008

\begin{tabular}{|c|c|c|c|c|c|c|c|}
\hline Measurement \# & Stnd Dev u & Stnd Dev v & Stnd Dev w & Mean SNR u & Mean SNR v & Mean SNR w & Mean Correlation \\
\hline & $\mathrm{cm} / \mathrm{s}$ & $\mathrm{cm} / \mathrm{s}$ & $\mathrm{cm} / \mathrm{s}$ & $\mathrm{dB}$ & $\mathrm{dB}$ & $\mathrm{dB}$ & $\%$ \\
\hline 56 & 0.39 & 0.35 & 0.27 & 6.14 & 6.51 & 5.77 & 89.48 \\
\hline 57 & 0.40 & 0.36 & 0.28 & 5.92 & 6.32 & 5.66 & 89.11 \\
\hline 58 & 0.37 & 0.34 & 0.27 & 5.78 & 6.17 & 5.49 & 88.79 \\
\hline 59 & 0.33 & 0.30 & 0.22 & 5.69 & 6.14 & 5.44 & 88.49 \\
\hline 60 & 0.38 & 0.32 & 0.20 & 5.34 & 5.72 & 5.13 & 87.57 \\
\hline 61 & 0.41 & 0.35 & 0.23 & 4.92 & 5.28 & 5.16 & 86.09 \\
\hline 62 & 0.38 & 0.38 & 0.24 & 4.90 & 5.17 & 5.02 & 85.62 \\
\hline 63 & 0.36 & 0.37 & 0.24 & 4.91 & 5.22 & 5.06 & 85.56 \\
\hline 64 & 0.41 & 0.36 & 0.26 & 5.34 & 5.07 & 4.56 & 85.21 \\
\hline 65 & 0.43 & 0.39 & 0.27 & 5.07 & 4.85 & 4.25 & 84.46 \\
\hline 66 & 0.43 & 0.40 & 0.29 & 5.11 & 4.90 & 4.32 & 84.49 \\
\hline 67 & 0.43 & 0.42 & 0.30 & 4.92 & 4.64 & 4.18 & 83.96 \\
\hline 68 & 0.45 & 0.39 & 0.35 & 4.95 & 4.64 & 4.15 & 83.80 \\
\hline 69 & 0.42 & 0.38 & 0.31 & 4.43 & 4.62 & 4.07 & 83.49 \\
\hline 70 & 0.39 & 0.34 & 0.31 & 4.48 & 4.65 & 4.11 & 83.70 \\
\hline 71 & 0.44 & 0.39 & 0.34 & 4.71 & 4.88 & 4.33 & 84.10 \\
\hline 72 & 0.49 & 0.40 & 0.34 & 5.91 & 6.32 & 5.63 & 86.65 \\
\hline 73 & 0.49 & 0.35 & 0.35 & 8.73 & 9.28 & 8.77 & 91.58 \\
\hline 74 & 0.50 & 0.43 & 0.35 & 7.95 & 8.48 & 7.98 & 90.35 \\
\hline 75 & 0.51 & 0.45 & 0.35 & 8.09 & 8.69 & 8.13 & 90.01 \\
\hline
\end{tabular}

Table 21: Reynolds Stress, TI, and RMS Measured on September 30, 2008

\begin{tabular}{|c|c|c|c|c|c|c|c|c|c|c|}
\hline Measurement \# & RS_uw & RS_vu & RS_wv & RS_uu & RS_vv & RS_ww & Urms & Vrms & Wrms & TI \\
\hline & $\mathrm{Pa}$ & $\mathrm{Pa}$ & $\mathrm{Pa}$ & $\mathrm{Pa}$ & $\mathrm{Pa}$ & $\mathrm{Pa}$ & $\mathrm{cm} / \mathrm{s}$ & $\mathrm{cm} / \mathrm{s}$ & $\mathrm{cm} / \mathrm{s}$ & \\
\hline 56 & $-1.58 \mathrm{E}-03$ & $2.85 \mathrm{E}-03$ & $1.29 \mathrm{E}-03$ & $-1.55 \mathrm{E}-02$ & $-1.25 \mathrm{E}-02$ & $-7.42 \mathrm{E}-03$ & 4.22E-01 & $3.32 \mathrm{E}-01$ & $2.24 \mathrm{E}-01$ & 1.77E-01 \\
\hline 57 & 2.47E-05 & $7.16 \mathrm{E}-04$ & $2.54 \mathrm{E}-04$ & $-1.61 \mathrm{E}-02$ & $-1.30 \mathrm{E}-02$ & $-7.60 \mathrm{E}-03$ & 4.22E- 01 & $4.21 \mathrm{E}-01$ & $2.86 \mathrm{E}-01$ & $1.82 \mathrm{E}-01$ \\
\hline 58 & $1.12 \mathrm{E}-03$ & $-7.12 \mathrm{E}-04$ & $3.42 \mathrm{E}-06$ & \begin{tabular}{|l|}
$-39 E-02$ \\
\end{tabular} & -1.17E-02 & -7.17E-03 & 4.70E-01 & $3.92 \mathrm{E}-01$ & $3.52 \mathrm{E}-01$ & 1.79E-01 \\
\hline 59 & $1.03 \mathrm{E}-03$ & $-1.91 \mathrm{E}-03$ & $-4.24 \mathrm{E}-04$ & $-1.09 \mathrm{E}-02$ & $-8.93 E-03$ & $-4.61 \mathrm{E}-03$ & $4.02 \mathrm{E}-01$ & $3.88 \mathrm{E}-01$ & $3.34 \mathrm{E}-01$ & 1.63E-01 \\
\hline 60 & $1.35 \mathrm{E}-03$ & $-2.66 \mathrm{E}-03$ & $8.21 \mathrm{E}-05$ & $-1.42 \mathrm{E}-02$ & $-1.01 E-02$ & $-4.01 E-03$ & 4.03E-01 & $3.66 \mathrm{E}-01$ & 2.57E-01 & 1.64E-01 \\
\hline 61 & $1.05 \mathrm{E}-04$ & $-3.68 \mathrm{E}-03$ & $9.46 \mathrm{E}-04$ & $-1.68 \mathrm{E}-02$ & $-1.21 \mathrm{E}-02$ & $-5.43 E-03$ & 4.45E-01 & 3.75E-01 & 2.97E-01 & 1.69E-01 \\
\hline 62 & $-1.57 \mathrm{E}-03$ & $-3.12 \mathrm{E}-03$ & $-1.31 \mathrm{E}-04$ & $-1.44 \mathrm{E}-02$ & $-1.41 \mathrm{E}-02$ & $-5.58 \mathrm{E}-03$ & 3.63E-01 & $3.24 \mathrm{E}-01$ & 2. $60 \mathrm{E}-01$ & $1.72 \mathrm{E}-01$ \\
\hline 63 & $-2.46 \mathrm{E}-03$ & $-5.14 \mathrm{E}-04$ & 8.47E-04 & $-1.32 \mathrm{E}-02$ & $-1.34 \mathrm{E}-02$ & $-5.91 \mathrm{E}-03$ & 2.67E-01 & $3.02 \mathrm{E}-01$ & $2.28 \mathrm{E}-01$ & 1.79E-01 \\
\hline 64 & $-1.02 \mathrm{E}-03$ & $1.52 \mathrm{E}-03$ & $4.89 \mathrm{E}-04$ & $-1.65 \mathrm{E}-02$ & $-1.31 \mathrm{E}-02$ & $-6.78 \mathrm{E}-03$ & 2.66E-01 & $2.79 \mathrm{E}-01$ & 2.09E-01 & $2.26 \mathrm{E}-01$ \\
\hline 65 & $-1.74 \mathrm{E}-03$ & $3.14 \mathrm{E}-03$ & 4.85E-04 & $-1.82 \mathrm{E}-02$ & $-1.51 \mathrm{E}-02$ & $-7.30 \mathrm{E}-03$ & 2.57E-01 & 2.87E-01 & 2.14E-01 & 2.21E-01 \\
\hline 66 & $-1.67 \mathrm{E}-03$ & 4.34E-03 & $-3.32 \mathrm{E}-04$ & $-1.83 \mathrm{E}-02$ & $-1.63 \mathrm{E}-02$ & $-8.58 \mathrm{E}-03$ & 2.63E-01 & $2.86 \mathrm{E}-01$ & $1.85 \mathrm{E}-01$ & 2.35E-01 \\
\hline 67 & $-3.41 \mathrm{E}-03$ & $3.06 \mathrm{E}-03$ & $9.49 \mathrm{E}-04$ & $-1.84 \mathrm{E}-02$ & $-1.76 \mathrm{E}-02$ & $-9.20 \mathrm{E}-03$ & 2.63E-01 & $2.75 \mathrm{E}-01$ & $1.75 \mathrm{E}-01$ & $2.60 \mathrm{E}-01$ \\
\hline 68 & $-3.82 \mathrm{E}-03$ & $3.32 \mathrm{E}-03$ & 4.31E-04 & $-2.03 E-02$ & $-1.55 \mathrm{E}-02$ & $-1.23 \mathrm{E}-02$ & $2.53 \mathrm{E}-01$ & $2.68 \mathrm{E}-01$ & $1.71 \mathrm{E}-01$ & 2.67E-01 \\
\hline 69 & $-3.31 \mathrm{E}-03$ & $8.11 \mathrm{E}-04$ & $1.19 \mathrm{E}-03$ & $-1.79 \mathrm{E}-02$ & $-1.41 \mathrm{E}-02$ & $-9.40 \mathrm{E}-03$ & 2.71E-01 & $2.32 \mathrm{E}-01$ & $2.12 \mathrm{E}-01$ & $2.78 \mathrm{E}-01$ \\
\hline 70 & $-1.40 \mathrm{E}-03$ & $-5.24 \mathrm{E}-04$ & $1.05 \mathrm{E}-03$ & $-1.50 \mathrm{E}-02$ & $-1.17 \mathrm{E}-02$ & $-9.88 \mathrm{E}-03$ & $2.51 \mathrm{E}-01$ & $2.73 \mathrm{E}-01$ & $1.86 \mathrm{E}-01$ & $2.75 \mathrm{E}-01$ \\
\hline 71 & $3.98 \mathrm{E}-03$ & $-1.02 \mathrm{E}-03$ & 9.71E-05 & $-1.92 \mathrm{E}-02$ & $-1.51 E-02$ & \begin{tabular}{|l|}
$-18 \mathrm{E}-02$ \\
\end{tabular} & 2.48E-01 & $2.58 \mathrm{E}-01$ & $1.93 \mathrm{E}-01$ & $2.88 \mathrm{E}-01$ \\
\hline 72 & $5.80 \mathrm{E}-03$ & $6.78 \mathrm{E}-04$ & $-6.69 \mathrm{E}-04$ & $-2.40 \mathrm{E}-02$ & $-1.60 \mathrm{E}-02$ & $-1.14 \mathrm{E}-02$ & 2.45E-01 & $2.45 \mathrm{E}-01$ & $1.85 \mathrm{E}-01$ & $2.54 \mathrm{E}-01$ \\
\hline 73 & $4.92 \mathrm{E}-03$ & $-9.20 E-04$ & $-2.96 \mathrm{E}-04$ & $-2.34 \mathrm{E}-02$ & $-1.24 \mathrm{E}-02$ & $-1.25 \mathrm{E}-02$ & $2.40 \mathrm{E}-01$ & $2.51 \mathrm{E}-01$ & 1.67E-01 & 2.19E-01 \\
\hline 74 & $6.01 \mathrm{E}-03$ & 7.93E-04 & $9.82 \mathrm{E}-04$ & $-2.51 \mathrm{E}-02$ & $-1.88 \mathrm{E}-02$ & $-1.20 \mathrm{E}-02$ & 2.77E-01 & 2.63E-01 & $2.16 \mathrm{E}-01$ & 1.94E-01 \\
\hline 75 & $4.62 \mathrm{E}-03$ & $4.86 \mathrm{E}-04$ & $-1.22 \mathrm{E}-03$ & $-2.63 \mathrm{E}-02$ & $-1.97 \mathrm{E}-02$ & $\mid-1.25 \mathrm{E}-02$ & 2.64E-01 & $2.80 \mathrm{E}-01$ & 2.07E-01 & $1.90 \mathrm{E}-01$ \\
\hline
\end{tabular}


Table 22: Velocity Measurements Take on October 9, 2008

\begin{tabular}{|c|c|c|c|c|}
\hline Date Measured & $10 / 9 / 2008$ & & Flow Rate(Gallons/s) & Flow Rate(cm^3/s) \\
\hline Water Temperature & $21.2^{\circ} \mathrm{C}$ & & 4.3 & 16236 \\
\hline Raceway & A2 & & 4.7 & 17663 \\
\hline Sample Time & 6 Minutes & & 4.4 & 16663 \\
\hline Sample Rate & $10 \mathrm{~Hz}$ & Average & 4.5 & 16854 \\
\hline Screen Size & Small $(\mathrm{clean})$ & & & \\
\hline QZ Length & $180 \mathrm{~cm}$ & & & \\
\hline QZ Width & $91 \mathrm{~cm}$ & & & \\
\hline QZ Water Depth & $89 \mathrm{~cm}$ & & & \\
\hline Measured Dist. from Wall & $45 \mathrm{~cm}$ & & & \\
\hline Measured Dist. from Start of QZ & $90 \mathrm{~cm}$ & & & \\
\hline Measured with & $\approx 5000$ Striped Bass & & & \\
\hline
\end{tabular}

Table 23: Mean Velocity Values Measured on October 9, 2008

\begin{tabular}{|c|c|c|c|c|}
\hline Measurement \# & Meas. Distance From Bottom & Mean u & Mean v & Mean w \\
\hline & $\mathbf{c m}$ & $\mathbf{c m} / \mathbf{s}$ & $\mathbf{c m} / \mathbf{s}$ & $\mathbf{c m} / \mathbf{s}$ \\
\hline 76 & 5.16 & 1.74 & -0.08 & 0.02 \\
\hline 77 & 7.70 & 1.90 & -0.09 & 0.00 \\
\hline 78 & 10.24 & 2.38 & 0.01 & -0.21 \\
\hline 79 & 12.78 & 2.54 & -0.11 & -0.23 \\
\hline 80 & 15.32 & 2.72 & -0.10 & -0.22 \\
\hline 81 & 17.86 & 2.89 & -0.20 & -0.25 \\
\hline 82 & 20.40 & 2.90 & -0.20 & -0.22 \\
\hline 83 & 22.94 & 2.95 & -0.20 & -0.24 \\
\hline 84 & 25.48 & 2.98 & -0.19 & -0.18 \\
\hline 85 & 28.02 & 2.95 & -0.15 & -0.10 \\
\hline 86 & 30.56 & 2.93 & -0.22 & -0.11 \\
\hline 87 & 33.10 & 2.97 & -0.21 & -0.11 \\
\hline 88 & 35.64 & 2.97 & -0.19 & -0.03 \\
\hline 89 & 38.18 & 3.03 & -0.25 & -0.03 \\
\hline 90 & 40.72 & 2.98 & -0.24 & 0.05 \\
\hline 91 & 43.26 & 2.96 & -0.18 & 0.03 \\
\hline 92 & 45.80 & 2.92 & -0.09 & 0.01 \\
\hline 93 & 48.34 & 2.82 & -0.09 & -0.07 \\
\hline 94 & 50.88 & 2.75 & -0.10 & -0.07 \\
\hline 95 & 53.42 & 2.76 & -0.18 & -0.06 \\
\hline 96 & 55.96 & 2.80 & -0.16 & -0.07 \\
\hline 97 & 58.50 & 2.77 & -0.12 & 0.02 \\
\hline 98 & 61.04 & 2.85 & 0.04 & -0.14 \\
\hline
\end{tabular}


Table 24: Standard Deviation and SNR Measured on October 9, 2008

\begin{tabular}{|c|c|c|c|c|c|c|c|}
\hline Measurement \# & Stnd Dev u & Stnd Dev v & Stnd Dev w & Mean SNR u & Mean SNR v & Mean SNR w & Mean Correlation \\
\hline & $\mathrm{cm} / \mathrm{s}$ & $\mathrm{cm} / \mathrm{s}$ & $\mathrm{cm} / \mathrm{s}$ & $\mathrm{dB}$ & $\mathrm{dB}$ & $\mathrm{dB}$ & $\%$ \\
\hline 76 & 0.31 & 0.31 & 0.15 & 10.16 & 11.24 & 9.94 & 94.68 \\
\hline 77 & 0.30 & 0.23 & 0.18 & 9.38 & 10.27 & 9.00 & 93.93 \\
\hline 78 & 0.32 & 0.27 & 0.23 & 10.29 & 10.84 & 9.96 & 94.16 \\
\hline 79 & 0.30 & 0.28 & 0.19 & 11.40 & 11.69 & 10.83 & 94.57 \\
\hline 80 & 0.29 & 0.24 & 0.18 & 10.28 & 10.35 & 9.52 & 93.64 \\
\hline 81 & 0.25 & 0.24 & 0.17 & 9.83 & 9.88 & 9.09 & 93.12 \\
\hline 82 & 0.25 & 0.21 & 0.14 & 10.19 & 10.10 & 9.30 & 93.39 \\
\hline 83 & 0.25 & 0.20 & 0.14 & 10.77 & 10.79 & 9.99 & 93.58 \\
\hline 84 & 0.27 & 0.20 & 0.13 & 8.82 & 9.65 & 8.46 & 92.63 \\
\hline 85 & 0.25 & 0.21 & 0.15 & 9.08 & 9.01 & 8.32 & 92.26 \\
\hline 86 & 0.26 & 0.22 & 0.14 & 8.82 & 8.82 & 8.17 & 92.00 \\
\hline 87 & 0.25 & 0.23 & 0.12 & 8.58 & 8.61 & 7.93 & 91.70 \\
\hline 88 & 0.26 & 0.23 & 0.13 & 8.22 & 8.20 & 7.53 & 91.43 \\
\hline 89 & 0.26 & 0.23 & 0.13 & 8.00 & 7.96 & 7.39 & 91.07 \\
\hline 90 & 0.27 & 0.24 & 0.14 & 6.51 & 7.82 & 6.60 & 88.92 \\
\hline 91 & 0.29 & 0.26 & 0.16 & 6.53 & 7.75 & 6.59 & 88.77 \\
\hline 92 & 0.30 & 0.23 & 0.16 & 6.66 & 7.88 & 6.69 & 89.00 \\
\hline 93 & 0.33 & 0.24 & 0.16 & 6.61 & 7.31 & 6.10 & 88.18 \\
\hline 94 & 0.29 & 0.23 & 0.16 & 7.29 & 8.23 & 7.31 & 89.78 \\
\hline 95 & 0.31 & 0.24 & 0.22 & 6.56 & 8.24 & 7.10 & 90.54 \\
\hline 96 & 0.31 & 0.25 & 0.21 & 8.48 & 9.06 & 7.86 & 90.94 \\
\hline 97 & 0.37 & 0.28 & 0.21 & 7.52 & 8.03 & 6.90 & 88.91 \\
\hline 98 & 0.32 & 0.27 & 0.21 & 8.02 & 8.55 & 7.38 & 90.23 \\
\hline
\end{tabular}


Table 25: Reynolds Stress, TI, and RMS Measured on October 9, 2008

\begin{tabular}{|c|c|c|c|c|c|c|c|c|c|c|}
\hline Measurement \# & RS_uw & RS_vu & RS_wv & RS_uu & RS_vv & RS_ww & Urms & Vrms & Wrms & TI \\
\hline & $\mathrm{Pa}$ & $\mathrm{Pa}$ & $\mathrm{Pa}$ & $\mathrm{Pa}$ & $\mathrm{Pa}$ & $\mathrm{Pa}$ & $\mathrm{cm} / \mathrm{s}$ & $\mathrm{cm} / \mathrm{s}$ & $\mathrm{cm} / \mathrm{s}$ & \\
\hline 76 & $1.40 \mathrm{E}-03$ & $-7.96 \mathrm{E}-05$ & $2.96 \mathrm{E}-04$ & $-9.81 E-03$ & $-9.37 E-03$ & $-2.32 \mathrm{E}-03$ & 3.14E-01 & 3.07E-01 & $1.52 \mathrm{E}-01$ & $1.54 \mathrm{E}-01$ \\
\hline 77 & $8.82 \mathrm{E}-04$ & $1.04 \mathrm{E}-03$ & $2.48 \mathrm{E}-04$ & $-9.15 E-03$ & $-5.39 E-03$ & $-3.26 \mathrm{E}-03$ & 3.03E-01 & 2.33E-01 & $1.81 \mathrm{E}-01$ & 1.29E-01 \\
\hline 78 & $3.29 \mathrm{E}-03$ & -9.03E-04 & $-7.86 \mathrm{E}-04$ & $-9.89 \mathrm{E}-03$ & $-7.12 \mathrm{E}-03$ & $-5.24 \mathrm{E}-03$ & 3.15E-01 & 2.67E-01 & $2.29 \mathrm{E}-01$ & 1.14E-01 \\
\hline 79 & $2.16 \mathrm{E}-03$ & 7.44E-04 & $-6.16 \mathrm{E}-04$ & $-8.84 \mathrm{E}-03$ & $-7.58 \mathrm{E}-03$ & $-3.52 E-03$ & 2.98E-01 & $2.76 \mathrm{E}-01$ & $1.88 \mathrm{E}-01$ & $1.01 \mathrm{E}-01$ \\
\hline 80 & $1.97 \mathrm{E}-03$ & $3.36 \mathrm{E}-04$ & $-5.71 \mathrm{E}-04$ & $-8.12 \mathrm{E}-03$ & $-5.68 \mathrm{E}-03$ & $-3.31 E-03$ & 2.86E-01 & 2.39E-01 & $1.82 \mathrm{E}-01$ & 8.77E-02 \\
\hline 81 & $2.94 \mathrm{E}-04$ & $-1.82 \mathrm{E}-04$ & $-1.37 \mathrm{E}-04$ & $-6.46 \mathrm{E}-03$ & $-5.79 E-03$ & $-2.77 \mathrm{E}-03$ & $2.55 \mathrm{E}-01$ & $2.41 \mathrm{E}-01$ & $1.67 \mathrm{E}-01$ & 7.70E-02 \\
\hline 82 & $-4.26 \mathrm{E}-04$ & $-7.12 E-04$ & $-2.21 \mathrm{E}-04$ & $-6.06 \mathrm{E}-03$ & $-4.35 E-03$ & $-1.89 \mathrm{E}-03$ & 2.47E-01 & $2.09 \mathrm{E}-01$ & $1.38 \mathrm{E}-01$ & $6.96 \mathrm{E}-02$ \\
\hline 83 & $-5.33 \mathrm{E}-04$ & $-1.07 \mathrm{E}-03$ & $-3.14 \mathrm{E}-05$ & $-6.19 \mathrm{E}-03$ & $-3.82 \mathrm{E}-03$ & $-1.91 \mathrm{E}-03$ & 2.49E-01 & $1.96 \mathrm{E}-01$ & $1.38 \mathrm{E}-01$ & $6.73 \mathrm{E}-02$ \\
\hline 84 & $-2.30 \mathrm{E}-04$ & $-1.27 \mathrm{E}-03$ & $1.87 \mathrm{E}-04$ & $-7.19 E-03$ & $-4.18 E-03$ & $-1.81 \mathrm{E}-03$ & 2.69E-01 & $2.05 \mathrm{E}-01$ & $1.35 \mathrm{E}-01$ & 7.02E-02 \\
\hline 85 & $-4.00 \mathrm{E}-04$ & $-9.40 E-04$ & $-2.70 \mathrm{E}-04$ & $-6.00 \mathrm{E}-03$ & $-4.43 E-03$ & $-2.30 \mathrm{E}-03$ & $2.45 \mathrm{E}-01$ & $2.11 \mathrm{E}-01$ & $1.52 \mathrm{E}-01$ & 7.00E-02 \\
\hline 86 & $-7.60 \mathrm{E}-05$ & $-1.50 \mathrm{E}-03$ & $-2.73 E-04$ & $-6.83 E-03$ & $-5.01 E-03$ & $-1.83 \mathrm{E}-03$ & 2.62E-01 & $2.24 \mathrm{E}-01$ & $1.35 \mathrm{E}-01$ & 7.28E-02 \\
\hline 87 & $2.40 \mathrm{E}-04$ & $-7.91 E-04$ & $1.11 \mathrm{E}-04$ & $-6.24 \mathrm{E}-03$ & $-5.14 \mathrm{E}-03$ & $-1.43 \mathrm{E}-03$ & $2.50 \mathrm{E}-01$ & 2.27E-01 & 1.20E-01 & $6.96 \mathrm{E}-02$ \\
\hline 88 & $-1.63 \mathrm{E}-04$ & $-1.40 \mathrm{E}-03$ & $-2.36 \mathrm{E}-04$ & $-6.52 E-03$ & $-5.25 \mathrm{E}-03$ & $-1.57 \mathrm{E}-03$ & $2.56 \mathrm{E}-01$ & $2.30 \mathrm{E}-01$ & $1.26 \mathrm{E}-01$ & $7.09 \mathrm{E}-02$ \\
\hline 89 & $-3.08 \mathrm{E}-04$ & $-5.23 E-04$ & $8.23 \mathrm{E}-05$ & $-6.67 \mathrm{E}-03$ & $-5.27 \mathrm{E}-03$ & $-1.64 \mathrm{E}-03$ & $2.59 \mathrm{E}-01$ & $2.30 \mathrm{E}-01$ & $1.28 \mathrm{E}-01$ & 7.01E-02 \\
\hline 90 & $-7.45 E-04$ & $-7.31 E-04$ & $1.36 \mathrm{E}-04$ & $-7.34 \mathrm{E}-03$ & $-5.88 \mathrm{E}-03$ & $-2.01 E-03$ & 2.71E-01 & $2.43 \mathrm{E}-01$ & $1.42 \mathrm{E}-01$ & 7.55E-02 \\
\hline 91 & $-5.63 E-04$ & $-7.83 E-04$ & $3.71 \mathrm{E}-04$ & $-8.54 \mathrm{E}-03$ & $-6.76 \mathrm{E}-03$ & $-2.52 E-03$ & 2.93E-01 & $2.61 \mathrm{E}-01$ & $1.59 \mathrm{E}-01$ & $8.24 \mathrm{E}-02$ \\
\hline 92 & $-7.44 \mathrm{E}-04$ & $-1.45 E-03$ & 4.17E-04 & $-8.78 \mathrm{E}-03$ & $-5.40 E-03$ & $-2.41 E-03$ & 2.97E-01 & $2.33 \mathrm{E}-01$ & $1.56 \mathrm{E}-01$ & $8.06 \mathrm{E}-02$ \\
\hline 93 & $-3.51 E-04$ & $-1.67 \mathrm{E}-03$ & $1.27 \mathrm{E}-04$ & $-1.07 \mathrm{E}-02$ & $-5.62 E-03$ & $-2.49 \mathrm{E}-03$ & $3.28 \mathrm{E}-01$ & $2.38 \mathrm{E}-01$ & $1.58 \mathrm{E}-01$ & $8.90 \mathrm{E}-02$ \\
\hline 94 & $3.61 \mathrm{E}-04$ & $-1.38 \mathrm{E}-03$ & $3.76 \mathrm{E}-04$ & $-8.66 \mathrm{E}-03$ & $-5.15 E-03$ & $-2.58 \mathrm{E}-03$ & $2.95 \mathrm{E}-01$ & 2.27E-01 & $1.61 \mathrm{E}-01$ & $8.52 E-02$ \\
\hline 95 & $-2.47 \mathrm{E}-04$ & $-8.98 E-04$ & $1.38 \mathrm{E}-04$ & $-9.43 E-03$ & $-5.58 E-03$ & $-4.68 E-03$ & $3.08 \mathrm{E}-01$ & 2.37E-01 & 2.17E-01 & $9.28 \mathrm{E}-02$ \\
\hline 96 & $-5.36 \mathrm{E}-05$ & $-1.33 \mathrm{E}-03$ & $-7.32 \mathrm{E}-04$ & $-9.60 \mathrm{E}-03$ & $-6.37 \mathrm{E}-03$ & $-4.42 \mathrm{E}-03$ & $3.10 \mathrm{E}-01$ & $2.53 \mathrm{E}-01$ & $2.11 \mathrm{E}-01$ & $9.29 \mathrm{E}-02$ \\
\hline 97 & $-2.92 \mathrm{E}-04$ & $-1.90 \mathrm{E}-03$ & $-1.60 \mathrm{E}-04$ & $-1.36 \mathrm{E}-02$ & $-7.73 E-03$ & $-4.40 \mathrm{E}-03$ & 3.70E-01 & 2.79E-01 & $2.10 \mathrm{E}-01$ & $1.06 \mathrm{E}-01$ \\
\hline 98 & 7.47E-05 & $-2.69 \mathrm{E}-03$ & -3.07E-04 & $-1.03 E-02$ & -7.47E-03 & $-4.30 \mathrm{E}-03$ & $3.21 \mathrm{E}-01$ & $2.74 \mathrm{E}-01$ & $2.08 \mathrm{E}-01$ & $9.51 \mathrm{E}-02$ \\
\hline
\end{tabular}


Table 26: Velocity Measurements Taken on November 4, 2008

\begin{tabular}{|c|c|c|c|c|}
\hline Date Measured & $11 / 4 / 2008$ & & Flow Rate(Gallons/s) & Flow Rate $\left(\mathrm{cm}^{\wedge} 3 / \mathrm{s}\right)$ \\
\hline Water Temperature & $17.2^{\circ} \mathrm{C}$ & & \begin{tabular}{|c|}
3.5 \\
\end{tabular} & \begin{tabular}{|c|}
13287 \\
\end{tabular} \\
\hline Raceway & A2 & & 3.5 & 13090 \\
\hline Sample Time & 6 Minutes & & 3.5 & 13143 \\
\hline Sample Rate & $10 \mathrm{~Hz}$ & Average & 3.5 & 13173 \\
\hline Screen Size & Small & & & \\
\hline QZ Length & $180 \mathrm{~cm}$ & & & \\
\hline QZ Width & $91 \mathrm{~cm}$ & & & \\
\hline QZ Water Depth & $89 \mathrm{~cm}$ & & & \\
\hline Measured Dist. from Bottom & $7.7 \mathrm{~cm}$ & & & \\
\hline Measured Dist. from Start of QZ & $90 \mathrm{~cm}$ & & & \\
\hline Measured with & $\approx 5000$ Striped Bass & & & \\
\hline
\end{tabular}


Table 27: Mean Velocity Values Measured on November 4, 2008

\begin{tabular}{|c|c|c|c|c|}
\hline Measurement \# & Meas. Distance From Side & Mean u & Mean v & Mean w \\
\hline & $\mathrm{cm}$ & $\mathrm{cm} / \mathrm{s}$ & $\mathrm{cm} / \mathrm{s}$ & $\mathrm{cm} / \mathrm{s}$ \\
\hline 99 & 7.62 & 0.79 & 0.06 & -0.39 \\
\hline 100 & 10.16 & 0.89 & -0.03 & -0.37 \\
\hline 101 & 12.70 & 0.82 & -0.10 & -0.06 \\
\hline 102 & 15.24 & 1.22 & -0.17 & -0.08 \\
\hline 103 & 17.78 & 1.24 & -0.25 & -0.07 \\
\hline 104 & 20.32 & 1.07 & -0.22 & 0.02 \\
\hline 105 & 22.86 & 1.27 & -0.12 & -0.12 \\
\hline 106 & 25.40 & 1.59 & -0.28 & -0.22 \\
\hline 107 & 27.94 & 1.34 & -0.21 & -0.03 \\
\hline 108 & 30.48 & 1.56 & -0.30 & -0.13 \\
\hline 109 & 33.02 & 1.65 & -0.25 & -0.12 \\
\hline 110 & 35.56 & 1.54 & -0.23 & -0.13 \\
\hline 111 & 38.10 & 1.87 & -0.25 & -0.25 \\
\hline 112 & 40.64 & 1.66 & -0.23 & -0.26 \\
\hline 113 & 43.18 & 1.76 & -0.30 & -0.29 \\
\hline 114 & 45.72 & 1.70 & -0.25 & -0.25 \\
\hline 115 & 48.26 & 1.71 & -0.17 & -0.19 \\
\hline 116 & 50.80 & 1.65 & -0.14 & -0.20 \\
\hline 117 & 53.34 & 1.47 & -0.15 & -0.11 \\
\hline 118 & 55.88 & 1.44 & -0.06 & -0.07 \\
\hline 119 & 58.42 & 1.40 & -0.13 & -0.08 \\
\hline 120 & 60.96 & 1.35 & -0.08 & -0.11 \\
\hline 121 & 63.50 & 1.18 & -0.11 & -0.04 \\
\hline 122 & 66.04 & 1.25 & -0.10 & -0.10 \\
\hline 123 & 68.58 & 1.21 & -0.03 & 0.17 \\
\hline 124 & 71.12 & 1.14 & 0.02 & 0.08 \\
\hline 125 & 73.66 & 1.10 & 0.01 & 0.05 \\
\hline 126 & 76.20 & 1.20 & -0.03 & 0.17 \\
\hline 127 & 78.74 & 1.28 & 0.01 & 0.17 \\
\hline 128 & 81.28 & 1.24 & -0.02 & 0.12 \\
\hline 129 & 83.82 & 1.28 & 0.01 & 0.21 \\
\hline
\end{tabular}


Table 28: Standard Deviation and SNR Measured on November 4, 2008

\begin{tabular}{|c|c|c|c|c|c|c|c|}
\hline Measurement \# & Stnd Dev u & Stnd Dev v & Stnd Dev w & Mean SNR u & Mean SNR v & Mean SNR w & Mean Correlation \\
\hline & $\mathrm{cm} / \mathrm{s}$ & $\mathrm{cm} / \mathrm{s}$ & $\mathrm{cm} / \mathrm{s}$ & \begin{tabular}{|c|}
$d B$ \\
\end{tabular} & $\mathrm{~dB}$ & $\mathrm{~dB}$ & $\%$ \\
\hline 99 & 0.36 & 0.31 & 0.41 & 9.80 & 9.97 & 8.43 & 94.36 \\
\hline 100 & 0.45 & 0.45 & 0.40 & 8.88 & 9.09 & 7.78 & 93.82 \\
\hline 101 & 0.42 & 0.41 & 0.36 & 9.09 & 9.30 & 8.01 & 93.94 \\
\hline 102 & 0.50 & 0.48 & 0.41 & 8.46 & 8.61 & 7.33 & 93.44 \\
\hline 103 & 0.47 & 0.50 & 0.41 & 8.36 & 8.49 & 7.29 & 93.38 \\
\hline 104 & 0.47 & 0.50 & 0.39 & 8.29 & 8.45 & 7.15 & 93.33 \\
\hline 105 & 0.53 & 0.52 & 0.38 & 7.82 & 8.30 & 6.92 & 93.07 \\
\hline 106 & 0.60 & 0.60 & 0.49 & 8.01 & 8.34 & 6.99 & 92.88 \\
\hline 107 & 0.50 & 0.49 & 0.42 & 7.56 & 8.15 & 6.88 & 92.92 \\
\hline 108 & 0.55 & 0.52 & 0.46 & 7.28 & 8.08 & 6.79 & 92.63 \\
\hline 109 & 0.54 & 0.53 & 0.46 & 7.04 & 7.74 & 6.42 & 92.25 \\
\hline 110 & 0.53 & 0.53 & 0.46 & 6.79 & 7.48 & 6.27 & 91.90 \\
\hline 111 & 0.54 & 0.49 & 0.39 & 7.52 & 7.45 & 6.32 & 90.77 \\
\hline 112 & 0.49 & 0.50 & 0.42 & 7.31 & 7.18 & 6.14 & 90.54 \\
\hline 113 & 0.47 & 0.44 & 0.39 & 7.25 & 7.18 & 6.10 & 90.44 \\
\hline 114 & 0.45 & 0.40 & 0.36 & 7.38 & 7.25 & 6.13 & 90.47 \\
\hline 115 & 0.43 & 0.40 & 0.32 & 7.24 & 7.18 & 6.02 & 90.60 \\
\hline 116 & 0.42 & 0.40 & 0.32 & 7.14 & 7.07 & 5.98 & 90.48 \\
\hline 117 & 0.36 & 0.33 & 0.25 & 6.99 & 6.77 & 5.76 & 90.32 \\
\hline 118 & 0.38 & 0.33 & 0.23 & 6.51 & 6.72 & 5.66 & 90.59 \\
\hline 119 & 0.34 & 0.33 & 0.25 & 6.07 & 6.56 & 5.48 & 90.76 \\
\hline 120 & 0.34 & 0.31 & 0.26 & 6.07 & 6.52 & 5.45 & 90.61 \\
\hline 121 & 0.39 & 0.31 & 0.23 & 6.18 & 6.81 & 5.72 & 90.89 \\
\hline 122 & 0.50 & 0.31 & 0.27 & 6.00 & 6.57 & 5.49 & 90.59 \\
\hline 123 & 0.41 & 0.36 & 0.32 & 5.96 & 6.60 & 5.47 & 90.52 \\
\hline 124 & 0.40 & 0.33 & 0.35 & 6.05 & 6.71 & 5.56 & 90.47 \\
\hline 125 & 0.38 & 0.38 & 0.34 & 5.83 & 6.45 & 5.35 & 90.24 \\
\hline 126 & 0.41 & 0.37 & 0.35 & 5.86 & 6.47 & 5.36 & 90.07 \\
\hline 127 & 0.44 & 0.37 & 0.33 & 5.77 & 6.38 & 5.24 & 89.85 \\
\hline 128 & 0.45 & 0.35 & 0.31 & 5.57 & 6.04 & 5.07 & 89.57 \\
\hline 129 & 0.40 & 0.36 & 0.33 & 5.44 & 6.05 & 5.00 & 89.35 \\
\hline
\end{tabular}


Table 29: Reynolds Stress, TI, and RMS Measured on November 4, 2008

\begin{tabular}{|c|c|c|c|c|c|c|c|c|c|c|}
\hline Measurement \# & RS_uw & RS_vu & RS_wv & RS_uu & RS_vv & RS_ww & Urms & Vrms & Wrms & TI \\
\hline & $\mathrm{Pa}$ & $\mathrm{Pa}$ & $\mathrm{Pa}$ & $\mathrm{Pa}$ & $\mathrm{Pa}$ & $\mathrm{Pa}$ & $\mathrm{cm} / \mathrm{s}$ & $\mathrm{cm} / \mathrm{s}$ & $\mathrm{cm} / \mathrm{s}$ & \\
\hline 99 & $1.07 \mathrm{E}-03$ & $-5.75 E-05$ & $2.84 \mathrm{E}-03$ & $-1.29 \mathrm{E}-02$ & $-9.55 E-03$ & $-1.69 \mathrm{E}-02$ & $3.60 \mathrm{E}-01$ & 3.10E-01 & $4.12 \mathrm{E}-01$ & $4.10 \mathrm{E}-01$ \\
\hline 100 & $2.39 \mathrm{E}-03$ & 4.23E-03 & 4.65E-03 & $-2.01 \mathrm{E}-02$ & $-2.00 \mathrm{E}-02$ & $-1.57 \mathrm{E}-02$ & 4.49E-01 & $4.48 \mathrm{E}-01$ & $3.98 \mathrm{E}-01$ & 4.47E-01 \\
\hline 101 & 4.57E-03 & $1.60 \mathrm{E}-03$ & $1.76 \mathrm{E}-03$ & $-1.78 \mathrm{E}-02$ & $-1.66 \mathrm{E}-02$ & $-1.32 \mathrm{E}-02$ & $23 \mathrm{E}-01$ & $4.08 \mathrm{E}-01$ & $.64 \mathrm{E}-01$ & $4.80 \mathrm{E}-01$ \\
\hline 102 & 7.87E-03 & $3.20 \mathrm{E}-03$ & $1.23 \mathrm{E}-03$ & $-2.53 \mathrm{E}-02$ & $-2.25 \mathrm{E}-02$ & $-1.65 \mathrm{E}-02$ & 5.04E-01 & 4.75E- 01 & 4.07E-01 & 3.75E-01 \\
\hline 103 & $7.76 \mathrm{E}-03$ & $4.31 \mathrm{E}-03$ & 7.85E-04 & $-2.21 \mathrm{E}-02$ & $-2.52 E-02$ & $-1.66 \mathrm{E}-02$ & 4.71E-01 & $5.03 \mathrm{E}-01$ & $4.08 \mathrm{E}-01$ & $3.64 \mathrm{E}$ \\
\hline 104 & $4.28 \mathrm{E}-03$ & 6.47E-03 & $1.28 \mathrm{E}-03$ & $-2.17 \mathrm{E}-02$ & $-2.50 \mathrm{E}-02$ & $-1.51 \mathrm{E}-02$ & $4.66 \mathrm{E}-01$ & $5.01 \mathrm{E}-01$ & $3.90 \mathrm{E}-01$ & \\
\hline 105 & $8.89 \mathrm{E}-03$ & $2.65 \mathrm{E}-03$ & $-1.31 \mathrm{E}-03$ & $-2.78 \mathrm{E}-02$ & $-2.68 \mathrm{E}-02$ & $-1.44 \mathrm{E}-02$ & $5.28 \mathrm{E}-01$ & $5.19 \mathrm{E}-01$ & $3.81 \mathrm{E}-01$ & $3.77 \mathrm{E}$ \\
\hline 106 & & 5.67E-03 & $-6.44 \mathrm{E}-04$ & $-3.61 \mathrm{E}-02$ & $-3.53 \mathrm{E}-02$ & $-2.43 \mathrm{E}-02$ & & 5.95E-01 & & 3.47E-01 \\
\hline 107 & 9.77E-03 & $-7.53 E-04$ & $1.22 \mathrm{E}-03$ & $-2.50 \mathrm{E}-02$ & $-2.43 E-02$ & $-1.73 \mathrm{E}-02$ & 5.01E-01 & & & \\
\hline 108 & 7.59E-03 & $2.27 \mathrm{E}-03$ & $2.70 \mathrm{E}-03$ & $-2.96 \mathrm{E}-02$ & $-2.73 \mathrm{E}-02$ & $-2.13 \mathrm{E}-02$ & 5.45E-01 & & & \\
\hline 109 & $1.34 \mathrm{E}-02$ & $2.78 \mathrm{E}-03$ & $-1.27 \mathrm{E}-03$ & $-2.85 E-02$ & $-2.85 E-02$ & $-2.15 \mathrm{E}-02$ & 5.35E-01 & $5.35 \mathrm{E}-01$ & & \\
\hline 110 & $9.95 \mathrm{E}-03$ & $6.22 \mathrm{E}-03$ & $-1.09 \mathrm{E}-03$ & $-2.84 \mathrm{E}-02$ & $-2.82 E-02$ & $-2.07 \mathrm{E}-02$ & 5.34E- 01 & $5.32 \mathrm{E}-01$ & $4.56 \mathrm{E}-01$ & \\
\hline 111 & $1.12 \mathrm{E}-02$ & $2.76 \mathrm{E}-03$ & $-8.63 \mathrm{E}-04$ & $-2.92 \mathrm{E}-02$ & $-2.44 \mathrm{E}-02$ & $-1.52 \mathrm{E}-02$ & 5.42E-01 & 4.95E-01 & $3.91 \mathrm{E}-01$ & \\
\hline 112 & $7.29 \mathrm{E}-03$ & $6.26 \mathrm{E}-04$ & $-1.38 \mathrm{E}-04$ & $-2.40 \mathrm{E}-02$ & $-2.44 \mathrm{E}-02$ & $-1.76 \mathrm{E}-02$ & 4.91E-01 & 4.95E-01 & $4.21 \mathrm{E}-01$ & 2.77E-01 \\
\hline 113 & $7.34 \mathrm{E}-03$ & $-1.18 \mathrm{E}-03$ & $6.03 \mathrm{E}-05$ & $-2.20 \mathrm{E}-02$ & $-1.96 \mathrm{E}-02$ & $-1.53 \mathrm{E}-02$ & 4.70E-01 & 4.44E-01 & $3.92 \mathrm{E}-01$ & $2.42 \mathrm{E}-01$ \\
\hline 114 & 4.70E-03 & $-1.63 \mathrm{E}-03$ & $3.64 \mathrm{E}-04$ & $-2.03 \mathrm{E}-02$ & $-1.58 \mathrm{E}-02$ & $-1.31 \mathrm{E}-02$ & $4.51 \mathrm{E}-01$ & $3.99 \mathrm{E}-01$ & $3.63 \mathrm{E}-01$ & $2.34 \mathrm{E}$ \\
\hline 115 & $5.23 \mathrm{E}-03$ & $-2.09 \mathrm{E}-04$ & $-4.32 \mathrm{E}-04$ & $-1.88 \mathrm{E}-02$ & $-1.56 \mathrm{E}-02$ & $-1.03 E-02$ & 4.34E- 01 & $3.96 \mathrm{E}-01$ & $3.22 \mathrm{E}-01$ & 2.24 \\
\hline 116 & $5.52 \mathrm{E}-03$ & $-1.50 \mathrm{E}-03$ & $9.44 \mathrm{E}-04$ & $-1.76 \mathrm{E}-02$ & $-1.57 \mathrm{E}-02$ & $-1.00 \mathrm{E}-02$ & $4.21 \mathrm{E}-01$ & 3.97E-01 & 3.17E-01 & $2.28 \mathrm{E}-01$ \\
\hline 117 & $3.03 \mathrm{E}-03$ & $-5.43 E-04$ & $-4.77 \mathrm{E}-06$ & $-1.26 \mathrm{E}-02$ & $-1.10 \mathrm{E}-02$ & $-6.32 \mathrm{E}-03$ & $3.56 \mathrm{E}-01$ & $3.33 \mathrm{E}-01$ & $2.52 \mathrm{E}-01$ & $2.14 \mathrm{E}-01$ \\
\hline 118 & $1.97 \mathrm{E}-03$ & $1.10 \mathrm{E}-03$ & $-1.44 \mathrm{E}-03$ & $-1.42 \mathrm{E}-02$ & -1.07E-02 & $-5.14 \mathrm{E}-03$ & 3.77E-01 & $3.28 \mathrm{E}-01$ & 2.27E-01 & $2.19 \mathrm{E}-01$ \\
\hline 119 & $2.03 \mathrm{E}-03$ & $-1.28 \mathrm{E}-03$ & $1.95 \mathrm{E}-04$ & $-1.14 \mathrm{E}-02$ & $-1.07 \mathrm{E}-02$ & $-6.06 \mathrm{E}-03$ & 3.39E-01 & $3.28 \mathrm{E}-01$ & 2.47E-01 & $2.19 \mathrm{E}-01$ \\
\hline 120 & $6.37 \mathrm{E}-04$ & 5.27E-04 & $2.70 \mathrm{E}-04$ & $-1.16 \mathrm{E}-02$ & $-9.79 E-03$ & $-6.64 \mathrm{E}-03$ & 3.41E-01 & $3.14 \mathrm{E}-01$ & $2.58 \mathrm{E}-01$ & $2.26 \mathrm{E}-01$ \\
\hline 121 & $3.80 \mathrm{E}-03$ & $1.95 \mathrm{E}-04$ & $-8.66 \mathrm{E}-05$ & $-1.49 \mathrm{E}-02$ & $-9.51 E-03$ & $-5.25 \mathrm{E}-03$ & 3.87E-01 & $3.09 \mathrm{E}-01$ & 2.30E-01 & 2.65E-01 \\
\hline 122 & 4.97E-04 & $-1.74 \mathrm{E}-03$ & $8.36 \mathrm{E}-04$ & $-2.46 \mathrm{E}-02$ & $-9.56 \mathrm{E}-03$ & $-7.08 \mathrm{E}-03$ & 4.97E-01 & $3.10 \mathrm{E}-01$ & 2.67E-01 & 2.95E-01 \\
\hline 123 & $-2.54 \mathrm{E}-03$ & $-1.34 \mathrm{E}-03$ & $-6.15 E-04$ & \begin{tabular}{|l|}
$-1.67 \mathrm{E}-02$ \\
\end{tabular} & $-1.27 \mathrm{E}-02$ & $-1.02 \mathrm{E}-02$ & 4.10E-01 & 3.57E-01 & $3.21 \mathrm{E}-01$ & 2.99E-01 \\
\hline 124 & $-3.53 E-03$ & -7.77E-04 & $-7.88 \mathrm{E}-04$ & $-1.57 \mathrm{E}-02$ & -1.07E-02 & $-1.20 \mathrm{E}-02$ & 3.97E-01 & $3.28 \mathrm{E}-01$ & $3.48 \mathrm{E}-01$ & 3.13E-01 \\
\hline 125 & $-3.16 E-03$ & $-9.00 \mathrm{E}-04$ & $-7.52 \mathrm{E}-04$ & $-1.42 \mathrm{E}-02$ & $-1.44 \mathrm{E}-02$ & $-1.17 \mathrm{E}-02$ & 3.77E-01 & $3.80 \mathrm{E}-01$ & $3.43 \mathrm{E}-01$ & 3.34E-01 \\
\hline 126 & $-3.25 \mathrm{E}-03$ & $2.58 \mathrm{E}-04$ & $1.58 \mathrm{E}-03$ & $-1.65 \mathrm{E}-02$ & $-1.39 \mathrm{E}-02$ & $-1.23 \mathrm{E}-02$ & 4.07E-01 & $3.74 \mathrm{E}-01$ & $3.51 \mathrm{E}-01$ & $3.12 \mathrm{E}-01$ \\
\hline 127 & $-5.41 E-03$ & $-1.47 \mathrm{E}-03$ & $-6.70 \mathrm{E}-04$ & $-1.90 \mathrm{E}-02$ & $-1.39 \mathrm{E}-02$ & $-1.10 \mathrm{E}-02$ & 4.37E-01 & $3.73 \mathrm{E}-01$ & 3.33E-01 & $2.96 \mathrm{E}-01$ \\
\hline 128 & $-2.98 \mathrm{E}-03$ & $-2.42 \mathrm{E}-04$ & $-1.03 \mathrm{E}-03$ & $-1.98 \mathrm{E}-02$ & $-1.21 \mathrm{E}-02$ & $-9.39 \mathrm{E}-03$ & 4.46E-01 & $3.49 \mathrm{E}-01$ & 3.07E-01 & $2.99 \mathrm{E}-01$ \\
\hline 129 & $-1.76 \mathrm{E}-03$ & $-2.62 E-03$ & $-2.77 \mathrm{E}-04$ & $-1.59 \mathrm{E}-02$ & $-1.31 \mathrm{E}-02$ & $-1.10 \mathrm{E}-02$ & $4.00 \mathrm{E}-01$ & 3.62E-01 & 3.32E-01 & $2.83 \mathrm{E}-01$ \\
\hline
\end{tabular}


Table 30: Velocity Measurements Taken on November 6, 2008

\begin{tabular}{|c|c|c|c|c|}
\hline Date Measured & $11 / 6 / 2008$ & & Flow Rate(Gallons/s) & Flow Rate $\left(\mathrm{cm}^{\wedge} 3 / \mathrm{s}\right)$ \\
\hline Water Temperature & $17.2^{\circ} \mathrm{C}$ & & \begin{tabular}{|c|}
3.6 \\
\end{tabular} & 13533 \\
\hline Raceway & A4 & & 3.5 & 13313 \\
\hline Sample Time & 6 Minutes & & 3.5 & 13169 \\
\hline Sample Rate & $10 \mathrm{~Hz}$ & Average & 3.5 & 13339 \\
\hline Screen Size & Large & & & \\
\hline QZ Length & $180 \mathrm{~cm}$ & & & \\
\hline QZ Width & $89 \mathrm{~cm}$ & & & \\
\hline QZ Water Depth & $89 \mathrm{~cm}$ & & & \\
\hline Measuring Dist. From Bottom & $7.7 \mathrm{~cm}$ & & & \\
\hline Measuring Dist. from Start of QZ & $90 \mathrm{~cm}$ & & & \\
\hline Measured with & No Fish & & & \\
\hline
\end{tabular}


Table 31: Mean Velocity Values Measured on November 6, 2008

\begin{tabular}{|c|c|c|c|c|}
\hline Measurement \# & Meas. Distance From Side & Mean u & Mean v & Mean w \\
\hline & $\mathrm{cm}$ & $\mathrm{cm} / \mathrm{s}$ & $\mathrm{cm} / \mathrm{s}$ & $\mathrm{cm} / \mathrm{s}$ \\
\hline 130 & 7.62 & 1.31 & 0.08 & -0.39 \\
\hline 131 & 10.16 & 1.32 & 0.11 & -0.37 \\
\hline 132 & 12.70 & 1.37 & 0.09 & -0.06 \\
\hline 133 & 15.24 & 1.51 & 0.04 & -0.08 \\
\hline 134 & 17.78 & 1.61 & -0.02 & -0.07 \\
\hline 135 & 20.32 & 1.44 & -0.07 & 0.02 \\
\hline 136 & 22.86 & 1.43 & -0.02 & -0.12 \\
\hline 137 & 25.40 & 1.54 & 0.06 & -0.22 \\
\hline 138 & 27.94 & 1.40 & 0.09 & -0.03 \\
\hline 139 & 30.48 & 1.71 & 0.22 & -0.13 \\
\hline 140 & 33.02 & 1.54 & 0.04 & -0.12 \\
\hline 141 & 35.56 & 1.50 & 0.04 & -0.13 \\
\hline 142 & 38.10 & 1.31 & 0.08 & -0.25 \\
\hline 143 & 40.64 & 1.56 & 0.15 & -0.26 \\
\hline 144 & 43.18 & 1.31 & 0.09 & -0.29 \\
\hline 145 & 45.72 & 1.49 & 0.27 & -0.25 \\
\hline 146 & 48.26 & 1.45 & 0.18 & -0.19 \\
\hline 147 & 50.80 & 1.39 & 0.13 & -0.20 \\
\hline 148 & 53.34 & 1.28 & 0.12 & -0.11 \\
\hline 149 & 55.88 & 1.31 & 0.08 & -0.07 \\
\hline 150 & 58.42 & 1.34 & 0.01 & -0.08 \\
\hline 151 & 60.96 & 1.11 & 0.04 & -0.11 \\
\hline 152 & 63.50 & 1.41 & 0.02 & -0.04 \\
\hline 153 & 66.04 & 1.48 & -0.10 & -0.10 \\
\hline 154 & 68.58 & 1.27 & 0.06 & 0.17 \\
\hline 155 & 71.12 & 1.38 & -0.11 & 0.08 \\
\hline 156 & 73.66 & 1.25 & 0.02 & 0.05 \\
\hline 157 & 76.20 & 1.24 & -0.06 & 0.17 \\
\hline 158 & 78.74 & 1.11 & 0.04 & 0.17 \\
\hline 159 & 81.28 & 1.15 & -0.03 & 0.12 \\
\hline
\end{tabular}


Table 32: Standard Deviation and SNR Measured on November 6, 2008

\begin{tabular}{|c|c|c|c|c|c|c|c|}
\hline Measurement \# & Stnd Dev u & Stnd Dev v & Stnd Dev w & Mean SNR u & Mean SNR v & Mean SNR w & Mean Correlation \\
\hline & $\mathrm{cm} / \mathrm{s}$ & $\mathrm{cm} / \mathrm{s}$ & $\mathrm{cm} / \mathrm{s}$ & \begin{tabular}{|c|}
$d B$ \\
\end{tabular} & $\mathrm{~dB}$ & $\mathrm{~dB}$ & $\%$ \\
\hline 99 & 0.36 & 0.31 & 0.41 & 9.80 & 9.97 & 8.43 & 94.36 \\
\hline 100 & 0.45 & 0.45 & 0.40 & 8.88 & 9.09 & 7.78 & 93.82 \\
\hline 101 & 0.42 & 0.41 & 0.36 & 9.09 & 9.30 & 8.01 & 93.94 \\
\hline 102 & 0.50 & 0.48 & 0.41 & 8.46 & 8.61 & 7.33 & 93.44 \\
\hline 103 & 0.47 & 0.50 & 0.41 & 8.36 & 8.49 & 7.29 & 93.38 \\
\hline 104 & 0.47 & 0.50 & 0.39 & 8.29 & 8.45 & 7.15 & 93.33 \\
\hline 105 & 0.53 & 0.52 & 0.38 & 7.82 & 8.30 & 6.92 & 93.07 \\
\hline 106 & 0.60 & 0.60 & 0.49 & 8.01 & 8.34 & 6.99 & 92.88 \\
\hline 107 & 0.50 & 0.49 & 0.42 & 7.56 & 8.15 & 6.88 & 92.92 \\
\hline 108 & 0.55 & 0.52 & 0.46 & 7.28 & 8.08 & 6.79 & 92.63 \\
\hline 109 & 0.54 & 0.53 & 0.46 & 7.04 & 7.74 & 6.42 & 92.25 \\
\hline 110 & 0.53 & 0.53 & 0.46 & 6.79 & 7.48 & 6.27 & 91.90 \\
\hline 111 & 0.54 & 0.49 & 0.39 & 7.52 & 7.45 & 6.32 & 90.77 \\
\hline 112 & 0.49 & 0.50 & 0.42 & 7.31 & 7.18 & 6.14 & 90.54 \\
\hline 113 & 0.47 & 0.44 & 0.39 & 7.25 & 7.18 & 6.10 & 90.44 \\
\hline 114 & 0.45 & 0.40 & 0.36 & 7.38 & 7.25 & 6.13 & 90.47 \\
\hline 115 & 0.43 & 0.40 & 0.32 & 7.24 & 7.18 & 6.02 & 90.60 \\
\hline 116 & 0.42 & 0.40 & 0.32 & 7.14 & 7.07 & 5.98 & 90.48 \\
\hline 117 & 0.36 & 0.33 & 0.25 & 6.99 & 6.77 & 5.76 & 90.32 \\
\hline 118 & 0.38 & 0.33 & 0.23 & 6.51 & 6.72 & 5.66 & 90.59 \\
\hline 119 & 0.34 & 0.33 & 0.25 & 6.07 & 6.56 & 5.48 & 90.76 \\
\hline 120 & 0.34 & 0.31 & 0.26 & 6.07 & 6.52 & 5.45 & 90.61 \\
\hline 121 & 0.39 & 0.31 & 0.23 & 6.18 & 6.81 & 5.72 & 90.89 \\
\hline 122 & 0.50 & 0.31 & 0.27 & 6.00 & 6.57 & 5.49 & 90.59 \\
\hline 123 & 0.41 & 0.36 & 0.32 & 5.96 & 6.60 & 5.47 & 90.52 \\
\hline 124 & 0.40 & 0.33 & 0.35 & 6.05 & 6.71 & 5.56 & 90.47 \\
\hline 125 & 0.38 & 0.38 & 0.34 & 5.83 & 6.45 & 5.35 & 90.24 \\
\hline 126 & 0.41 & 0.37 & 0.35 & 5.86 & 6.47 & 5.36 & 90.07 \\
\hline 127 & 0.44 & 0.37 & 0.33 & 5.77 & 6.38 & 5.24 & 89.85 \\
\hline 128 & 0.45 & 0.35 & 0.31 & 5.57 & 6.04 & 5.07 & 89.57 \\
\hline 129 & 0.40 & 0.36 & 0.33 & 5.44 & 6.05 & 5.00 & 89.35 \\
\hline
\end{tabular}


Table 33: Reynolds Stress, TI, and RMS Measured on November 6, 2008

\begin{tabular}{|c|c|c|c|c|c|c|c|c|c|c|}
\hline Measurement \# & RS_uw & RS_vu & RS_wv & RS_uu & RS_vv & RS_ww & Urms & Vrms & Wrms & TI \\
\hline & $\mathrm{Pa}$ & $\mathrm{Pa}$ & $\mathrm{Pa}$ & $\mathrm{Pa}$ & $\mathrm{Pa}$ & $\mathrm{Pa}$ & $\mathrm{cm} / \mathrm{s}$ & $\mathrm{cm} / \mathrm{s}$ & $\mathrm{cm} / \mathrm{s}$ & \\
\hline 130 & $2.68 \mathrm{E}-03$ & $1.69 \mathrm{E}-03$ & $8.02 \mathrm{E}-04$ & $-1.14 \mathrm{E}-02$ & $-6.18 \mathrm{E}-03$ & $-7.01 E-03$ & $3.39 \mathrm{E}-01$ & $2.49 \mathrm{E}-01$ & $2.65 \mathrm{E}-01$ & 2.17E-01 \\
\hline 131 & 1.71E-03 & $2.01 \mathrm{E}-03$ & 8.73E-04 & $-1.22 \mathrm{E}-02$ & $-6.26 \mathrm{E}-03$ & $-5.20 \mathrm{E}-03$ & $3.50 \mathrm{E}-01$ & $2.51 \mathrm{E}-01$ & 2.29E-01 & $2.10 \mathrm{E}-01$ \\
\hline 132 & $2.58 \mathrm{E}-03$ & $3.34 \mathrm{E}-03$ & $5.89 \mathrm{E}-04$ & $-1.25 \mathrm{E}-02$ & $-8.13 E-03$ & $-7.24 \mathrm{E}-03$ & $3.54 \mathrm{E}-01$ & $2.86 \mathrm{E}-01$ & $2.70 \mathrm{E}-01$ & $2.20 \mathrm{E}-01$ \\
\hline 133 & $4.22 \mathrm{E}-03$ & $-4.72 E-04$ & $2.08 \mathrm{E}-03$ & $-1.23 \mathrm{E}-02$ & $-9.01 E-03$ & $-7.78 \mathrm{E}-03$ & $3.51 \mathrm{E}-01$ & $3.01 \mathrm{E}-01$ & $2.80 \mathrm{E}-01$ & 2.05E-01 \\
\hline 134 & $2.11 \mathrm{E}-03$ & 7.45E-04 & $-3.56 \mathrm{E}-04$ & $-8.82 \mathrm{E}-03$ & -7.09E-03 & $-3.84 \mathrm{E}-03$ & $2.98 \mathrm{E}-01$ & 2.67E-01 & $1.96 \mathrm{E}-01$ & 1.60E-01 \\
\hline 135 & 3.75E-03 & $5.70 \mathrm{E}-04$ & $-5.72 E-04$ & $-1.36 \mathrm{E}-02$ & $-1.05 E-02$ & $-5.82 \mathrm{E}-03$ & $3.70 \mathrm{E}-01$ & $3.24 \mathrm{E}-01$ & $2.42 \mathrm{E}-01$ & 2.19E-01 \\
\hline 136 & 3.19E-03 & $-4.89 \mathrm{E}-04$ & $4.08 \mathrm{E}-04$ & $-1.24 \mathrm{E}-02$ & $-1.15 E-02$ & $-7.02 E-03$ & $3.53 \mathrm{E}-01$ & $3.40 \mathrm{E}-01$ & $2.66 \mathrm{E}-01$ & 2. $25 \mathrm{E}-01$ \\
\hline 137 & $3.59 \mathrm{E}-03$ & $1.21 \mathrm{E}-03$ & $6.79 \mathrm{E}-04$ & $-1.61 \mathrm{E}-02$ & $-1.19 E-02$ & $-6.74 \mathrm{E}-03$ & 4.03E-01 & $3.46 \mathrm{E}-01$ & $2.60 \mathrm{E}-01$ & $2.21 \mathrm{E}-01$ \\
\hline 138 & 4.45E-03 & $-1.13 \mathrm{E}-03$ & 7.07E-04 & $-1.47 \mathrm{E}-02$ & $-1.11 E-02$ & $-6.91 \mathrm{E}-03$ & $3.84 \mathrm{E}-01$ & 3.34E-01 & 2.63E-01 & $2.35 \mathrm{E}-01$ \\
\hline 139 & 4.75E-03 & $-4.10 \mathrm{E}-04$ & $-7.34 \mathrm{E}-04$ & $-1.46 \mathrm{E}-02$ & $-8.41 E-03$ & $-7.63 \mathrm{E}-03$ & 3.83E-01 & 2.91E-01 & 2.77E-01 & $1.86 \mathrm{E}-01$ \\
\hline 140 & $2.08 \mathrm{E}-03$ & 5.87E-04 & $-1.67 \mathrm{E}-03$ & $-1.35 \mathrm{E}-02$ & -1.57E-02 & $-7.11 \mathrm{E}-03$ & $3.68 \mathrm{E}-01$ & 3.97E-01 & 2.67E-01 & 2.27E-01 \\
\hline 141 & 2.37E-03 & $-4.15 E-04$ & $-1.59 E-04$ & $-1.35 \mathrm{E}-02$ & $-8.40 \mathrm{E}-03$ & $-6.14 \mathrm{E}-03$ & $3.69 \mathrm{E}-01$ & 2.90E-01 & $2.48 \mathrm{E}-01$ & 2.04E-01 \\
\hline 142 & $5.32 \mathrm{E}-03$ & $-1.38 \mathrm{E}-03$ & $-6.66 \mathrm{E}-04$ & $-1.66 \mathrm{E}-02$ & $-7.36 \mathrm{E}-03$ & $-9.18 \mathrm{E}-03$ & $4.08 \mathrm{E}-01$ & $2.72 \mathrm{E}-01$ & $3.04 \mathrm{E}-01$ & $2.54 \mathrm{E}-01$ \\
\hline 143 & $2.57 \mathrm{E}-03$ & $-5.23 E-04$ & $-6.42 E-04$ & $-1.14 \mathrm{E}-02$ & $-1.31 \mathrm{E}-02$ & $-6.48 \mathrm{E}-03$ & $3.38 \mathrm{E}-01$ & 3.63E-01 & $2.55 \mathrm{E}-01$ & $2.06 \mathrm{E}-01$ \\
\hline 144 & $4.01 \mathrm{E}-03$ & $-4.32 E-03$ & $1.65 \mathrm{E}-03$ & $-1.58 \mathrm{E}-02$ & $-1.66 \mathrm{E}-02$ & $-7.94 \mathrm{E}-03$ & 3.99E-01 & $4.09 \mathrm{E}-01$ & $2.82 \mathrm{E}-01$ & $2.80 \mathrm{E}-01$ \\
\hline 145 & $2.11 \mathrm{E}-03$ & $-2.29 \mathrm{E}-03$ & $-2.16 \mathrm{E}-03$ & $-1.41 \mathrm{E}-02$ & $-1.71 \mathrm{E}-02$ & $-8.91 \mathrm{E}-03$ & $3.76 \mathrm{E}-01$ & 4.15E-01 & 2.99E-01 & $2.43 \mathrm{E}-01$ \\
\hline 146 & 3.73E-03 & $-2.90 \mathrm{E}-03$ & $-9.75 E-04$ & $-1.48 \mathrm{E}-02$ & $-1.36 \mathrm{E}-02$ & $-8.43 E-03$ & 3.85E-01 & $3.70 \mathrm{E}-01$ & 2.91E-01 & $2.41 \mathrm{E}-01$ \\
\hline 147 & $4.64 \mathrm{E}-03$ & $-1.75 E-03$ & $-1.87 E-04$ & $-1.76 \mathrm{E}-02$ & $-1.85 E-02$ & $-7.60 \mathrm{E}-03$ & $4.21 \mathrm{E}-01$ & $4.31 \mathrm{E}-01$ & $2.76 \mathrm{E}-01$ & 2.73E-01 \\
\hline 148 & $3.04 \mathrm{E}-03$ & $-3.10 \mathrm{E}-03$ & $-6.07 E-05$ & $-1.32 \mathrm{E}-02$ & $-1.46 \mathrm{E}-02$ & $-8.00 \mathrm{E}-03$ & 3.64E-01 & $3.83 \mathrm{E}-01$ & 2.83E-01 & 2.69E-01 \\
\hline 149 & 3.47E-03 & $2.05 \mathrm{E}-03$ & $-3.65 E-04$ & $-1.37 \mathrm{E}-02$ & $-2.33 \mathrm{E}-02$ & $-8.78 \mathrm{E}-03$ & 3.71E-01 & 4.84E-01 & 2.97E-01 & 2.97E-01 \\
\hline 150 & 1.49E-03 & $4.31 \mathrm{E}-04$ & $2.73 \mathrm{E}-05$ & $-1.10 \mathrm{E}-02$ & $-1.26 \mathrm{E}-02$ & $-6.13 \mathrm{E}-03$ & 3.32E-01 & $3.56 \mathrm{E}-01$ & $2.48 \mathrm{E}-01$ & $2.36 \mathrm{E}-01$ \\
\hline 151 & 1.19E-03 & $-1.04 \mathrm{E}-03$ & $-1.86 \mathrm{E}-03$ & $-1.06 \mathrm{E}-02$ & $-1.29 \mathrm{E}-02$ & $-7.06 \mathrm{E}-03$ & $3.26 \mathrm{E}-01$ & $3.61 \mathrm{E}-01$ & $2.66 \mathrm{E}-01$ & 2.87E-01 \\
\hline 152 & $5.48 \mathrm{E}-03$ & $1.98 \mathrm{E}-03$ & $-5.92 E-04$ & $-1.66 \mathrm{E}-02$ & $-2.11 E-02$ & $-9.38 \mathrm{E}-03$ & $4.09 \mathrm{E}-01$ & $4.61 \mathrm{E}-01$ & $3.07 \mathrm{E}-01$ & $2.82 \mathrm{E}-01$ \\
\hline 153 & $3.49 \mathrm{E}-03$ & $2.09 \mathrm{E}-03$ & $-1.00 \mathrm{E}-03$ & $-1.42 \mathrm{E}-02$ & $-1.26 \mathrm{E}-02$ & $-7.34 \mathrm{E}-03$ & $3.78 \mathrm{E}-01$ & $3.56 \mathrm{E}-01$ & 2.71E-01 & $2.28 \mathrm{E}-01$ \\
\hline 154 & $-5.62 E-03$ & $2.38 \mathrm{E}-03$ & $7.22 \mathrm{E}-04$ & $-2.64 \mathrm{E}-02$ & $-1.24 \mathrm{E}-02$ & $-7.53 \mathrm{E}-03$ & $5.15 \mathrm{E}-01$ & $3.53 \mathrm{E}-01$ & $2.75 \mathrm{E}-01$ & $3.11 \mathrm{E}-01$ \\
\hline 155 & $-2.45 E-03$ & $-2.34 \mathrm{E}-03$ & $3.84 \mathrm{E}-04$ & $-1.57 \mathrm{E}-02$ & $-1.19 E-02$ & $-7.62 E-03$ & 3.97E-01 & $3.46 \mathrm{E}-01$ & $2.77 \mathrm{E}-01$ & 2.47E-01 \\
\hline 156 & $-4.24 \mathrm{E}-03$ & $4.28 \mathrm{E}-04$ & $1.12 \mathrm{E}-03$ & $-1.89 \mathrm{E}-02$ & $-1.10 \mathrm{E}-02$ & $-8.66 \mathrm{E}-03$ & 4.35E-01 & $3.33 \mathrm{E}-01$ & 2.95E-01 & 2.87E-01 \\
\hline 157 & $-4.19 E-03$ & $9.62 \mathrm{E}-04$ & $2.04 \mathrm{E}-03$ & $-1.67 \mathrm{E}-02$ & $-1.03 E-02$ & $-6.88 \mathrm{E}-03$ & $4.09 \mathrm{E}-01$ & $3.22 \mathrm{E}-01$ & 2.63E-01 & 2.71E-01 \\
\hline 158 & $-9.03 E-04$ & $-2.63 E-03$ & $9.51 E-04$ & $-1.44 \mathrm{E}-02$ & $-1.19 \mathrm{E}-02$ & $-7.48 \mathrm{E}-03$ & $3.80 \mathrm{E}-01$ & $3.46 \mathrm{E}-01$ & $2.74 \mathrm{E}-01$ & $3.00 \mathrm{E}-01$ \\
\hline 159 & -1.83E-03 & -1.87E-03 & $1.05 \mathrm{E}-03$ & $-1.16 \mathrm{E}-02$ & $-1.06 \mathrm{E}-02$ & $-1.04 \mathrm{E}-02$ & $3.41 \mathrm{E}-01$ & 3.27E-01 & 3.23E-01 & $2.83 \mathrm{E}-01$ \\
\hline
\end{tabular}


Table 34: Velocity Measurements Taken on November 26, 2008

\begin{tabular}{|c|c|c|c|c|}
\hline Date Measured & $11 / 26 / 2008$ & & Flow Rate(Gallons/s) & Flow Rate(cm^3/s) \\
\hline Water Temperature & $13.0^{\circ} \mathrm{C}$ & & 2.8 & 10735 \\
\hline Raceway & $\mathrm{A} 2$ & & 3.0 & 11273 \\
\hline Sample Time & $6 \mathrm{Minutes}$ & & 2.8 & 10709 \\
\hline Sample Rate & $10 \mathrm{~Hz}$ & Average & 2.9 & 10906 \\
\hline Screen Size & Small & & & \\
\hline QZ Length & $180 \mathrm{~cm}$ & & & \\
\hline QZ Width & $91 \mathrm{~cm}$ & & & \\
\hline QZ Water Depth & $89 \mathrm{~cm}$ & & & \\
\hline Measuring Dist. from Bottom & $7.7 \mathrm{~cm}$ & & & \\
\hline Measuring Dist. From Side of QZ & $45 \mathrm{~cm}$ & & & \\
\hline Measured with & $\approx 5000$ Striped Bass & & & \\
\hline
\end{tabular}

Table 35: Mean Velocity Values Measured on November 26, 2008

\begin{tabular}{|c|c|c|c|c|}
\hline Measurement \# & Meas. Distance from Start of QZ & Mean u & Mean v & Mean w \\
\hline & cm & $\mathrm{cm} / \mathrm{s}$ & $\mathrm{cm} / \mathrm{s}$ & $\mathrm{cm} / \mathrm{s}$ \\
\hline 160 & 58.42 & 0.47 & 0.04 & -0.38 \\
\hline 161 & 60.96 & 0.36 & 0.06 & -0.33 \\
\hline 162 & 63.50 & 0.37 & 0.24 & -0.25 \\
\hline 163 & 66.04 & 0.48 & 0.18 & -0.24 \\
\hline 164 & 68.58 & 0.65 & 0.14 & -0.45 \\
\hline 165 & 71.12 & 0.48 & 0.25 & -0.33 \\
\hline 166 & 73.66 & 0.60 & 0.27 & -0.41 \\
\hline 167 & 76.20 & 0.58 & 0.09 & -0.12 \\
\hline 168 & 78.74 & 0.63 & 0.22 & -0.19 \\
\hline 169 & 81.28 & 0.57 & 0.19 & -0.15 \\
\hline 170 & 83.82 & 0.57 & 0.15 & -0.12 \\
\hline 171 & 86.36 & 0.59 & 0.26 & -0.24 \\
\hline 172 & 88.90 & 0.76 & 0.09 & -0.19 \\
\hline 173 & 91.44 & 0.83 & 0.14 & -0.23 \\
\hline 174 & 93.98 & 0.72 & 0.14 & -0.14 \\
\hline 175 & 96.52 & 0.80 & 0.16 & -0.08 \\
\hline 176 & 99.06 & 0.75 & 0.18 & -0.17 \\
\hline 177 & 101.60 & 0.62 & 0.05 & -0.12 \\
\hline 178 & 104.14 & 0.76 & 0.12 & -0.18 \\
\hline 179 & 106.68 & 0.68 & 0.09 & -0.13 \\
\hline 180 & 109.22 & 0.52 & 0.07 & 0.00 \\
\hline 181 & 111.76 & 0.49 & -0.01 & -0.01 \\
\hline 182 & 114.30 & 0.67 & 0.01 & -0.06 \\
\hline
\end{tabular}


Table 36: Standard Deviation and SNR Measured on November 26, 2008

\begin{tabular}{|c|c|c|c|c|c|c|c|}
\hline Measurement \# & Stnd Dev u & Stnd Dev v & Stnd Dev w & Mean SNR u & Mean SNR v & Mean SNR w & Mean Correlation \\
\hline & $\mathrm{cm} / \mathrm{s}$ & $\mathrm{cm} / \mathrm{s}$ & $\mathrm{cm} / \mathrm{s}$ & \begin{tabular}{|c|}
$d B$ \\
\end{tabular} & $\mathrm{~dB}$ & $\mathrm{~dB}$ & $\%$ \\
\hline 160 & 0.45 & 0.52 & 0.46 & 6.99 & 7.78 & 6.59 & 92.15 \\
\hline 161 & 0.44 & 0.46 & 0.39 & 6.69 & 7.38 & 6.17 & 91.68 \\
\hline 162 & 0.40 & 0.41 & 0.38 & 6.65 & 7.22 & 6.01 & 91.52 \\
\hline 163 & 0.43 & 0.51 & 0.43 & 6.91 & 7.25 & 6.11 & 91.53 \\
\hline 164 & 0.51 & 0.44 & 0.47 & 6.64 & 7.13 & 5.95 & 91.11 \\
\hline 165 & 0.38 & 0.48 & 0.39 & 6.55 & 7.03 & 5.86 & 91.18 \\
\hline 166 & 0.45 & 0.43 & 0.42 & 6.45 & 6.94 & 5.75 & 90.89 \\
\hline 167 & 0.41 & 0.48 & 0.38 & 6.35 & 6.68 & 5.66 & 90.84 \\
\hline 168 & 0.48 & 0.45 & 0.41 & 6.22 & 6.53 & 5.44 & 90.45 \\
\hline 169 & 0.45 & 0.40 & 0.38 & 6.55 & 6.42 & 5.48 & 89.77 \\
\hline 170 & 0.43 & 0.45 & 0.41 & 7.34 & 6.54 & 5.63 & 88.86 \\
\hline 171 & 0.44 & 0.42 & 0.34 & 7.15 & 6.42 & 5.50 & 88.63 \\
\hline 172 & 0.43 & 0.41 & 0.28 & 7.10 & 6.30 & 5.43 & 88.61 \\
\hline 173 & 0.43 & 0.48 & 0.34 & 6.94 & 6.23 & 5.34 & 88.33 \\
\hline 174 & 0.45 & 0.45 & 0.40 & 6.81 & 6.14 & 5.27 & 88.01 \\
\hline 175 & 0.43 & 0.43 & 0.36 & 6.62 & 5.91 & 5.03 & 87.73 \\
\hline 176 & 0.40 & 0.45 & 0.40 & 3.96 & 5.33 & 4.42 & 88.15 \\
\hline 177 & 0.49 & 0.42 & 0.34 & 3.69 & 5.19 & 4.26 & 88.14 \\
\hline 178 & 0.43 & 0.42 & 0.38 & 3.53 & 5.14 & 4.20 & 87.93 \\
\hline 179 & 0.40 & 0.43 & 0.33 & 3.66 & 5.26 & 4.31 & 88.13 \\
\hline 180 & 0.36 & 0.35 & 0.36 & 3.43 & 5.01 & 4.03 & 87.65 \\
\hline 181 & 0.36 & 0.44 & 0.33 & 5.38 & 5.27 & 4.37 & 87.33 \\
\hline 182 & 0.42 & 0.44 & 0.34 & 5.33 & 5.17 & 4.17 & 86.62 \\
\hline
\end{tabular}


Table 37: Reynolds Stress, TI, and RMS Measured on November 26, 2008

\begin{tabular}{|c|c|c|c|c|c|c|c|c|c|c|}
\hline Measurement \# & RS_uw & RS_vu & RS_wv & RS_uu & RS_vv & RS_ww & Urms & Vrms & Wrms & TI \\
\hline & $\mathrm{Pa}$ & $\mathrm{Pa}$ & $\mathrm{Pa}$ & $\mathrm{Pa}$ & $\mathrm{Pa}$ & $\mathrm{Pa}$ & $\mathrm{cm} / \mathrm{s}$ & $\mathrm{cm} / \mathrm{s}$ & $\mathrm{cm} / \mathrm{s}$ & \\
\hline 160 & 7.15E-03 & $-2.25 \mathrm{E}-03$ & $-9.21 E-05$ & $-2.04 \mathrm{E}-02$ & $-2.72 E-02$ & $-2.14 \mathrm{E}-02$ & $4.52 \mathrm{E}-01$ & $5.22 \mathrm{E}-01$ & 4.63E-01 & 7.89E-01 \\
\hline 161 & $5.84 \mathrm{E}-03$ & $1.89 \mathrm{E}-04$ & $9.00 \mathrm{E}-04$ & $-1.92 \mathrm{E}-02$ & $-2.10 \mathrm{E}-02$ & $-1.55 \mathrm{E}-02$ & 4.39E-01 & 4.59E-01 & $3.94 \mathrm{E}-01$ & $8.71 \mathrm{E}-01$ \\
\hline 162 & $4.86 \mathrm{E}-03$ & $3.00 \mathrm{E}-03$ & $-2.68 \mathrm{E}-03$ & $-1.62 \mathrm{E}-02$ & $-1.70 \mathrm{E}-02$ & $-1.45 \mathrm{E}-02$ & 4.04E-01 & 4.13E-01 & 3.82E-01 & $7.94 \mathrm{E}-01$ \\
\hline 163 & $6.91 \mathrm{E}-03$ & $-1.71 \mathrm{E}-03$ & $-4.94 \mathrm{E}-03$ & $-1.85 \mathrm{E}-02$ & $-2.60 \mathrm{E}-02$ & $-1.82 \mathrm{E}-02$ & 4.30E-01 & 5.11E-01 & $4.28 \mathrm{E}-01$ & 8.19E-01 \\
\hline 164 & $1.30 \mathrm{E}-02$ & $5.43 \mathrm{E}-03$ & $-5.10 \mathrm{E}-03$ & $-2.63 \mathrm{E}-02$ & -1.97E-02 & $-2.17 \mathrm{E}-02$ & 5.14E-01 & 4.45E-01 & 4.67E-01 & 5.93E-01 \\
\hline 165 & $4.58 \mathrm{E}-03$ & $6.00 \mathrm{E}-04$ & $4.95 \mathrm{E}-04$ & $-1.41 \mathrm{E}-02$ & $-2.25 \mathrm{E}-02$ & $-1.55 \mathrm{E}-02$ & 3.76E-01 & 4.75E-01 & 3.95E-01 & $6.58 \mathrm{E}-01$ \\
\hline 166 & $7.06 \mathrm{E}-03$ & $2.68 \mathrm{E}-04$ & $-5.70 \mathrm{E}-03$ & $-2.04 \mathrm{E}-02$ & $-1.85 \mathrm{E}-02$ & $-1.77 \mathrm{E}-02$ & $4.53 \mathrm{E}-01$ & 4.31E-01 & $4.21 \mathrm{E}-01$ & $5.60 \mathrm{E}-01$ \\
\hline 167 & $4.74 \mathrm{E}-03$ & $-1.96 \mathrm{E}-03$ & $-2.13 \mathrm{E}-03$ & $-1.64 \mathrm{E}-02$ & $-2.32 \mathrm{E}-02$ & $-1.40 \mathrm{E}-02$ & 4.06E-01 & 4.82E-01 & $3.75 \mathrm{E}-01$ & 7.09E-01 \\
\hline 168 & $6.53 \mathrm{E}-03$ & $5.89 \mathrm{E}-05$ & $-4.00 \mathrm{E}-03$ & $-2.29 \mathrm{E}-02$ & $-1.99 E-02$ & $-1.67 \mathrm{E}-02$ & $4.80 \mathrm{E}-01$ & 4.47E-01 & 4.09E-01 & $6.43 \mathrm{E}-01$ \\
\hline 169 & $8.20 \mathrm{E}-03$ & $-2.32 E-04$ & $-9.97 \mathrm{E}-04$ & $-1.99 \mathrm{E}-02$ & $-1.62 \mathrm{E}-02$ & $-1.47 \mathrm{E}-02$ & $4.46 \mathrm{E}-01$ & 4.03E-01 & $3.84 \mathrm{E}-01$ & $6.65 \mathrm{E}-01$ \\
\hline 170 & 7.07E-03 & $-3.34 \mathrm{E}-03$ & $4.56 \mathrm{E}-04$ & $-1.83 \mathrm{E}-02$ & $-2.01 E-02$ & $-1.65 \mathrm{E}-02$ & $4.28 \mathrm{E}-01$ & 4.49E-01 & $4.06 \mathrm{E}-01$ & 7.18E-01 \\
\hline 171 & $7.05 \mathrm{E}-03$ & $-5.57 \mathrm{E}-04$ & $-9.67 \mathrm{E}-04$ & $-1.95 \mathrm{E}-02$ & $-1.79 \mathrm{E}-02$ & $-1.18 \mathrm{E}-02$ & 4.43E- 01 & 4.24E- 01 & $3.45 \mathrm{E}-01$ & $5.92 \mathrm{E}-01$ \\
\hline 172 & $4.26 \mathrm{E}-03$ & $7.23 \mathrm{E}-04$ & $-1.97 \mathrm{E}-03$ & $-1.88 \mathrm{E}-02$ & $-1.69 \mathrm{E}-02$ & $-7.70 \mathrm{E}-03$ & 4.34E-01 & 4.12E-01 & $2.78 \mathrm{E}-01$ & $4.85 \mathrm{E}-01$ \\
\hline 173 & $4.22 \mathrm{E}-03$ & $-1.99 E-03$ & $-3.39 E-04$ & $-1.84 \mathrm{E}-02$ & $-2.32 \mathrm{E}-02$ & $-1.15 \mathrm{E}-02$ & 4.30E-01 & 4.82E- 01 & $3.39 \mathrm{E}-01$ & 4.83E-01 \\
\hline 174 & 7.47E-03 & $-1.73 E-03$ & $-1.26 \mathrm{E}-04$ & $-2.00 \mathrm{E}-02$ & $-2.00 \mathrm{E}-02$ & $-1.57 \mathrm{E}-02$ & $4.48 \mathrm{E}-01$ & 4.48E-01 & 3.97E-01 & $5.81 \mathrm{E}-01$ \\
\hline 175 & $4.81 E-03$ & $-3.22 \mathrm{E}-04$ & $-3.38 \mathrm{E}-03$ & $-1.85 \mathrm{E}-02$ & $-1.84 \mathrm{E}-02$ & $-1.30 \mathrm{E}-02$ & 4.31E-01 & 4.30E-01 & $3.61 \mathrm{E}-01$ & 4.99E-01 \\
\hline 176 & $6.52 \mathrm{E}-03$ & 4.99E-03 & $-4.42 \mathrm{E}-03$ & $-1.60 \mathrm{E}-02$ & $-2.02 E-02$ & $-1.62 \mathrm{E}-02$ & 4.00E-01 & 4.50E-01 & 4.03E-01 & $5.29 \mathrm{E}-01$ \\
\hline 177 & $4.78 \mathrm{E}-03$ & 2.67E-03 & $-3.20 \mathrm{E}-03$ & $-2.41 \mathrm{E}-02$ & $-1.74 \mathrm{E}-02$ & $-1.14 \mathrm{E}-02$ & $4.92 \mathrm{E}-01$ & 4.18E-01 & $3.38 \mathrm{E}-01$ & $6.66 \mathrm{E}-01$ \\
\hline 178 & $8.15 E-03$ & $-1.81 \mathrm{E}-03$ & $-2.13 \mathrm{E}-04$ & $-1.81 \mathrm{E}-02$ & $-1.79 \mathrm{E}-02$ & $-1.47 \mathrm{E}-02$ & 4.27E-01 & 4.23E-01 & $3.85 \mathrm{E}-01$ & $5.21 \mathrm{E}-01$ \\
\hline 179 & 4.29E-03 & $-3.68 \mathrm{E}-03$ & $-2.82 E-03$ & $-1.62 \mathrm{E}-02$ & $-1.88 \mathrm{E}-02$ & $-1.05 E-02$ & 4.03E-01 & 4.34E- 01 & $3.25 \mathrm{E}-01$ & 5.59E-01 \\
\hline 180 & $4.26 \mathrm{E}-03$ & 5.47E-04 & -1.77E-03 & $-1.30 \mathrm{E}-02$ & $-1.21 \mathrm{E}-02$ & $-1.30 \mathrm{E}-02$ & $3.62 \mathrm{E}-01$ & $3.49 \mathrm{E}-01$ & $3.62 \mathrm{E}-01$ & $6.79 \mathrm{E}-01$ \\
\hline 181 & $4.43 E-03$ & $-3.46 E-04$ & $-1.32 \mathrm{E}-03$ & $-1.29 \mathrm{E}-02$ & $-1.94 \mathrm{E}-02$ & $-1.07 \mathrm{E}-02$ & 3.59E-01 & $4.41 \mathrm{E}-01$ & $3.28 \mathrm{E}-01$ & 7.76E-01 \\
\hline 182 & 5.97E-03 & $-2.53 E-04$ & $-3.09 E-03$ & $-1.79 \mathrm{E}-02$ & $-1.95 E-02$ & $-1.12 \mathrm{E}-02$ & 4.24E-01 & 4.43E-01 & 3.35E-01 & $6.04 \mathrm{E}-01$ \\
\hline
\end{tabular}


Table 38: Velocity Measurements Taken on November 29, 2008

\begin{tabular}{|c|c|c|c|c|}
\hline Date Measured & $11 / 29 / 2008$ & & Flow Rate(Gallons/s) & Flow Rate(cm^3/s) \\
\hline Water Temperature & $13.0^{\circ} \mathrm{C}$ & & 3.9 & 14687 \\
\hline Raceway & B4 & & 3.8 & 14479 \\
\hline Sample Time & 6 Minutes & Average & 3.9 & 14583 \\
\hline Sample Rate & $10 \mathrm{~Hz}$ & & & \\
\hline Screen Size & Large & & & \\
\hline QZ Length & $180 \mathrm{~cm}$ & & & \\
\hline QZ Width & $89 \mathrm{~cm}$ & & & \\
\hline QZ Water Depth & $89 \mathrm{~cm}$ & & & \\
\hline Measuring Dist. from Bottom & $7.7 \mathrm{~cm}$ & & & \\
\hline Measuring Dist. From Side of QZ & $45 \mathrm{~cm}$ & & & \\
\hline Measured with & No Fish & & & \\
\hline
\end{tabular}

Table 39: Mean Velocity Values Measured on November 29, 2008

\begin{tabular}{|c|c|c|c|c|}
\hline Measurement \# & Meas. Distance from Start of QZ & Mean u & Mean v & Mean w \\
\hline & cm & $\mathrm{cm} / \mathrm{s}$ & $\mathrm{cm} / \mathrm{s}$ & $\mathrm{cm} / \mathrm{s}$ \\
\hline 183 & 58.42 & 2.21 & 0.29 & -0.17 \\
\hline 184 & 60.96 & 2.26 & 0.39 & -0.14 \\
\hline 185 & 63.50 & 2.13 & 0.39 & -0.14 \\
\hline 186 & 66.04 & 2.37 & 0.34 & -0.20 \\
\hline 187 & 68.58 & 2.21 & 0.42 & -0.14 \\
\hline 188 & 71.12 & 2.31 & 0.30 & -0.18 \\
\hline 189 & 73.66 & 2.25 & 0.31 & -0.19 \\
\hline 190 & 76.20 & 2.19 & 0.28 & -0.13 \\
\hline 191 & 78.74 & 2.21 & 0.40 & -0.17 \\
\hline 192 & 81.28 & 2.08 & 0.09 & -0.12 \\
\hline 193 & 83.82 & 2.22 & 0.46 & -0.13 \\
\hline 194 & 86.36 & 2.17 & 0.29 & -0.10 \\
\hline 195 & 88.90 & 2.12 & 0.32 & -0.06 \\
\hline 196 & 91.44 & 2.10 & 0.42 & -0.07 \\
\hline 197 & 93.98 & 2.14 & 0.29 & -0.06 \\
\hline 198 & 96.52 & 2.12 & 0.24 & -0.09 \\
\hline 199 & 99.06 & 2.13 & 0.32 & -0.05 \\
\hline 200 & 101.60 & 2.21 & 0.47 & -0.09 \\
\hline 201 & 104.14 & 1.88 & 0.33 & 0.02 \\
\hline 202 & 106.68 & 1.97 & 0.34 & -0.04 \\
\hline 203 & 109.22 & 1.94 & 0.22 & -0.02 \\
\hline 204 & 111.76 & 2.00 & 0.40 & -0.07 \\
\hline 205 & 114.30 & 1.99 & 0.27 & -0.04 \\
\hline 206 & 116.84 & 1.85 & 0.24 & 0.02 \\
\hline 207 & 119.38 & 1.73 & 0.35 & 0.03 \\
\hline
\end{tabular}


Table 40: Standard Deviation and SNR Measured on November 29, 2008

\begin{tabular}{|c|c|c|c|c|c|c|c|}
\hline Measurement \# & Stnd Dev u & Stnd Dev v & Stnd Dev w & Mean SNR u & Mean SNR v & Mean SNR w & Mean Correlation \\
\hline & $\mathrm{cm} / \mathrm{s}$ & $\mathrm{cm} / \mathrm{s}$ & $\mathrm{cm} / \mathrm{s}$ & $\mathrm{dB}$ & $\mathrm{dB}$ & dB & $\%$ \\
\hline 183 & 0.43 & 0.45 & 0.31 & 7.67 & 8.57 & 7.37 & 92.66 \\
\hline 184 & 0.43 & 0.40 & 0.31 & 7.72 & 8.67 & 7.42 & 92.70 \\
\hline 185 & 0.48 & 0.49 & 0.35 & 7.64 & 8.55 & 7.31 & 92.65 \\
\hline 186 & 0.45 & 0.40 & 0.29 & 7.72 & 8.66 & 7.42 & 92.66 \\
\hline 187 & 0.51 & 0.39 & 0.35 & 7.48 & 8.39 & 7.21 & 92.49 \\
\hline 188 & 0.44 & 0.40 & 0.26 & 7.73 & 8.71 & 7.46 & 92.72 \\
\hline 189 & 0.43 & 0.39 & 0.31 & 7.45 & 8.36 & 7.11 & 92.49 \\
\hline 190 & 0.46 & 0.37 & 0.30 & 7.31 & 8.23 & 7.01 & 92.43 \\
\hline 191 & 0.41 & 0.42 & 0.38 & 8.07 & 9.07 & 7.75 & 92.83 \\
\hline 192 & 0.42 & 0.48 & 0.32 & 7.69 & 8.67 & 7.42 & 92.79 \\
\hline 193 & 0.50 & 0.47 & 0.34 & 7.28 & 8.25 & 6.98 & 92.33 \\
\hline 194 & 0.41 & 0.45 & 0.27 & 7.29 & 8.31 & 7.08 & 92.50 \\
\hline 195 & 0.51 & 0.38 & 0.32 & 8.44 & 8.66 & 7.61 & 91.26 \\
\hline 196 & 0.44 & 0.40 & 0.33 & 8.33 & 8.46 & 7.45 & 91.10 \\
\hline 197 & 0.38 & 0.39 & 0.30 & 6.30 & 7.65 & 6.28 & 90.67 \\
\hline 198 & 0.45 & 0.39 & 0.27 & 6.41 & 7.70 & 6.32 & 90.73 \\
\hline 199 & 0.44 & 0.40 & 0.31 & 6.15 & 7.52 & 6.07 & 90.27 \\
\hline 200 & 0.36 & 0.41 & 0.27 & 4.98 & 6.12 & 4.88 & 87.86 \\
\hline 201 & 0.43 & 0.46 & 0.27 & 4.59 & 5.82 & 4.58 & 87.18 \\
\hline 202 & 0.40 & 0.35 & 0.22 & 4.51 & 5.80 & 4.48 & 87.22 \\
\hline 203 & 0.44 & 0.41 & 0.28 & 3.55 & 5.55 & 4.10 & 88.42 \\
\hline 204 & 0.38 & 0.41 & 0.26 & 3.42 & 5.40 & 3.97 & 88.29 \\
\hline 205 & 0.38 & 0.37 & 0.22 & 3.52 & 5.55 & 4.14 & 88.46 \\
\hline 206 & 0.42 & 0.39 & 0.23 & 3.42 & 5.42 & 4.02 & 88.30 \\
\hline 207 & 0.43 & 0.43 & 0.23 & 3.43 & 5.45 & 4.03 & 88.33 \\
\hline
\end{tabular}


Table 41: Reynolds Stress, TI, and RMS Measured on November 29, 2008

\begin{tabular}{|c|c|c|c|c|c|c|c|c|c|c|}
\hline Measurement \# & RS_uw & RS_vu & RS_wv & RS_uu & RS_vv & RS_ww & Urms & Vrms & Wrms & TI \\
\hline & $\mathrm{Pa}$ & $\mathrm{Pa}$ & $\mathrm{Pa}$ & $\mathrm{Pa}$ & $\mathrm{Pa}$ & $\mathrm{Pa}$ & $\mathrm{cm} / \mathrm{s}$ & $\mathrm{cm} / \mathrm{s}$ & $\mathrm{cm} / \mathrm{s}$ & \\
\hline 183 & 4.57E-03 & $-3.91 \mathrm{E}-03$ & $2.34 \mathrm{E}-03$ & $-1.81 \mathrm{E}-02$ & $-2.00 \mathrm{E}-02$ & $-9.51 \mathrm{E}-03$ & $4.26 \mathrm{E}-01$ & $4.48 \mathrm{E}-01$ & $3.09 \mathrm{E}-01$ & $1.78 \mathrm{E}-01$ \\
\hline 184 & $5.02 \mathrm{E}-03$ & $1.14 \mathrm{E}-03$ & $2.17 \mathrm{E}-03$ & $-1.86 \mathrm{E}-02$ & $-1.61 \mathrm{E}-02$ & $-9.42 \mathrm{E}-03$ & 4.33E-01 & $4.02 \mathrm{E}-01$ & $3.08 \mathrm{E}-01$ & 1.67E-01 \\
\hline 185 & $7.25 \mathrm{E}-03$ & $-6.53 \mathrm{E}-04$ & -2.17E-05 & $-2.26 \mathrm{E}-02$ & $-2.44 \mathrm{E}-02$ & $-1.22 \mathrm{E}-02$ & 4.76E-01 & 4.95E-01 & $3.50 \mathrm{E}-01$ & $2.06 \mathrm{E}-01$ \\
\hline 186 & $4.10 \mathrm{E}-03$ & $-1.72 \mathrm{E}-03$ & $2.68 \mathrm{E}-03$ & $-2.00 \mathrm{E}-02$ & $-1.59 \mathrm{E}-02$ & $-8.42 \mathrm{E}-03$ & $4.48 \mathrm{E}-01$ & $4.00 \mathrm{E}-01$ & $2.91 \mathrm{E}-01$ & $1.60 \mathrm{E}-01$ \\
\hline 187 & $6.06 \mathrm{E}-03$ & $1.24 \mathrm{E}-03$ & $3.32 \mathrm{E}-03$ & $-2.54 \mathrm{E}-02$ & $-1.54 \mathrm{E}-02$ & $-1.23 \mathrm{E}-02$ & 5.05E-01 & 3.93E-01 & $3.51 \mathrm{E}-01$ & 1.87E-01 \\
\hline 188 & $3.62 \mathrm{E}-03$ & $-4.34 \mathrm{E}-03$ & $2.18 \mathrm{E}-03$ & $-1.96 \mathrm{E}-02$ & $-1.63 \mathrm{E}-02$ & $-6.63 E-03$ & 4.44E- 01 & 4.05E-01 & $2.58 \mathrm{E}-01$ & 1. $61 \mathrm{E}-01$ \\
\hline 189 & 4.44E-03 & $6.48 \mathrm{E}-04$ & $6.90 \mathrm{E}-04$ & $-1.88 \mathrm{E}-02$ & $-1.55 \mathrm{E}-02$ & $-9.48 \mathrm{E}-03$ & 4.35E-01 & 3.95E-01 & $3.08 \mathrm{E}-01$ & $1.68 \mathrm{E}-01$ \\
\hline 190 & $4.54 \mathrm{E}-03$ & $-1.66 \mathrm{E}-03$ & $2.08 \mathrm{E}-03$ & $-2.11 \mathrm{E}-02$ & $-1.36 \mathrm{E}-02$ & $-9.23 E-03$ & 4.61E-01 & $3.69 \mathrm{E}-01$ & $3.04 \mathrm{E}-01$ & $1.74 \mathrm{E}-01$ \\
\hline 191 & 4.33E-03 & 1.37E-03 & $1.83 \mathrm{E}-03$ & $-1.67 \mathrm{E}-02$ & $-1.75 E-02$ & $-1.42 \mathrm{E}-02$ & $4.10 \mathrm{E}-01$ & 4.19E-01 & $3.78 \mathrm{E}-01$ & 1.79E-01 \\
\hline 192 & $4.43 \mathrm{E}-03$ & $-2.08 \mathrm{E}-03$ & $-4.06 \mathrm{E}-04$ & $-1.76 \mathrm{E}-02$ & $-2.29 \mathrm{E}-02$ & $-1.04 \mathrm{E}-02$ & $4.21 \mathrm{E}-01$ & $4.80 \mathrm{E}-01$ & $3.23 \mathrm{E}-01$ & \\
\hline 193 & $6.30 \mathrm{E}-03$ & $-4.91 E-03$ & $6.02 \mathrm{E}-04$ & $-2.47 \mathrm{E}-02$ & $-2.24 \mathrm{E}-02$ & $-1.15 \mathrm{E}-02$ & 4.98E-01 & 4.74E-01 & $3.40 \mathrm{E}-01$ & \\
\hline 194 & 3.05E-03 & $-4.41 E-03$ & $9.22 \mathrm{E}-04$ & $-1.64 \mathrm{E}-02$ & $-2.03 E-02$ & $-7.34 \mathrm{E}-03$ & $4.06 \mathrm{E}-01$ & $4.52 \mathrm{E}-01$ & $2.72 \mathrm{E}-01$ & 1.75E-01 \\
\hline & & & & $-2.60 \mathrm{E}-02$ & $-1.45 E-02$ & $-1.02 \mathrm{E}-02$ & 5.11E-01 & $3.81 \mathrm{E}-01$ & $3.20 \mathrm{E}-01$ & \\
\hline 196 & $5.56 \mathrm{E}-03$ & $-3.72 \mathrm{E}-03$ & $1.99 \mathrm{E}-03$ & $-1.96 \mathrm{E}-02$ & $-1.60 \mathrm{E}-02$ & $-1.07 \mathrm{E}-02$ & 4.44E- 01 & 4.01E-01 & 3.29E-01 & \\
\hline 197 & $3.41 \mathrm{E}-03$ & $-1.53 E-03$ & $8.42 \mathrm{E}-04$ & $-1.42 \mathrm{E}-02$ & $-1.49 \mathrm{E}-02$ & $-8.70 \mathrm{E}-03$ & 3.78E-01 & 3.87E-01 & $2.96 \mathrm{E}-01$ & 1.65E-01 \\
\hline 198 & 3.89E-03 & $3.56 \mathrm{E}-05$ & $1.92 \mathrm{E}-03$ & $-1.97 \mathrm{E}-02$ & $-1.55 \mathrm{E}-02$ & $-7.53 \mathrm{E}-03$ & $4.45 \mathrm{E}-01$ & $3.94 \mathrm{E}-01$ & $2.75 \mathrm{E}-01$ & 1.77E-01 \\
\hline 199 & 4.32E-03 & $-4.04 \mathrm{E}-03$ & $2.83 \mathrm{E}-03$ & $-1.89 \mathrm{E}-02$ & $-1.56 \mathrm{E}-02$ & $-9.42 \mathrm{E}-03$ & 4.36E-01 & $3.96 \mathrm{E}-01$ & $3.08 \mathrm{E}-01$ & $1.78 \mathrm{E}-01$ \\
\hline 200 & $2.06 \mathrm{E}-03$ & $-3.88 \mathrm{E}-04$ & $-8.17 \mathrm{E}-04$ & $-1.31 \mathrm{E}-02$ & $-1.67 \mathrm{E}-02$ & $-7.40 \mathrm{E}-03$ & $3.62 \mathrm{E}-01$ & 4.10E-01 & $2.73 \mathrm{E}-01$ & $1.56 \mathrm{E}-01$ \\
\hline 201 & 3.25E-03 & $-3.43 E-03$ & $6.95 \mathrm{E}-04$ & $-1.80 \mathrm{E}-02$ & $-2.11 \mathrm{E}-02$ & $-7.38 \mathrm{E}-03$ & $4.25 \mathrm{E}-01$ & 4.60E-01 & $2.72 \mathrm{E}-01$ & $2.06 \mathrm{E}-01$ \\
\hline 202 & $2.10 \mathrm{E}-03$ & $-3.95 E-03$ & $1.76 \mathrm{E}-03$ & $-1.61 \mathrm{E}-02$ & $-1.23 \mathrm{E}-02$ & $-4.85 E-03$ & $4.03 \mathrm{E}-01$ & $3.52 \mathrm{E}-01$ & $2.21 \mathrm{E}-01$ & 1.67E-01 \\
\hline 203 & $3.74 \mathrm{E}-03$ & $-3.33 E-03$ & $1.18 \mathrm{E}-03$ & $-1.94 \mathrm{E}-02$ & $-1.68 \mathrm{E}-02$ & $-7.64 \mathrm{E}-03$ & 4.41E-01 & 4.11E-01 & 2.77E-01 & $1.96 \mathrm{E}-01$ \\
\hline 204 & $2.10 \mathrm{E}-03$ & $-1.33 E-03$ & $-7.30 \mathrm{E}-04$ & $-1.44 \mathrm{E}-02$ & $-1.65 \mathrm{E}-02$ & $-6.91 \mathrm{E}-03$ & 3.81E-01 & 4.07E-01 & 2.63E-01 & $1.75 \mathrm{E}-01$ \\
\hline 205 & $1.78 \mathrm{E}-03$ & $-2.41 \mathrm{E}-05$ & $9.69 \mathrm{E}-04$ & $-1.42 \mathrm{E}-02$ & $-1.38 \mathrm{E}-02$ & $-4.96 \mathrm{E}-03$ & 3.78E-01 & $3.72 \mathrm{E}-01$ & $2.23 \mathrm{E}-01$ & $1.65 \mathrm{E}-01$ \\
\hline 206 & $3.21 \mathrm{E}-03$ & $-3.93 E-03$ & 8.95E-04 & $-1.77 \mathrm{E}-02$ & $-1.54 \mathrm{E}-02$ & $-5.12 \mathrm{E}-03$ & $4.22 \mathrm{E}-01$ & 3.93E-01 & 2.27E-01 & $1.91 \mathrm{E}-01$ \\
\hline 207 & $2.23 \mathrm{E}-03$ & $-3.12 \mathrm{E}-03$ & $6.58 \mathrm{E}-04$ & $-1.80 \mathrm{E}-02$ & $-1.87 \mathrm{E}-02$ & $-5.39 E-03$ & 4.25E-01 & 4.33E-01 & $2.33 \mathrm{E}-01$ & 2.13E-01 \\
\hline
\end{tabular}




\section{Appendix B: Mean Velocity Profiles with Standard Deviation}

This appendix contains the individual plots and standard deviation from the data gathered on the corresponding days.

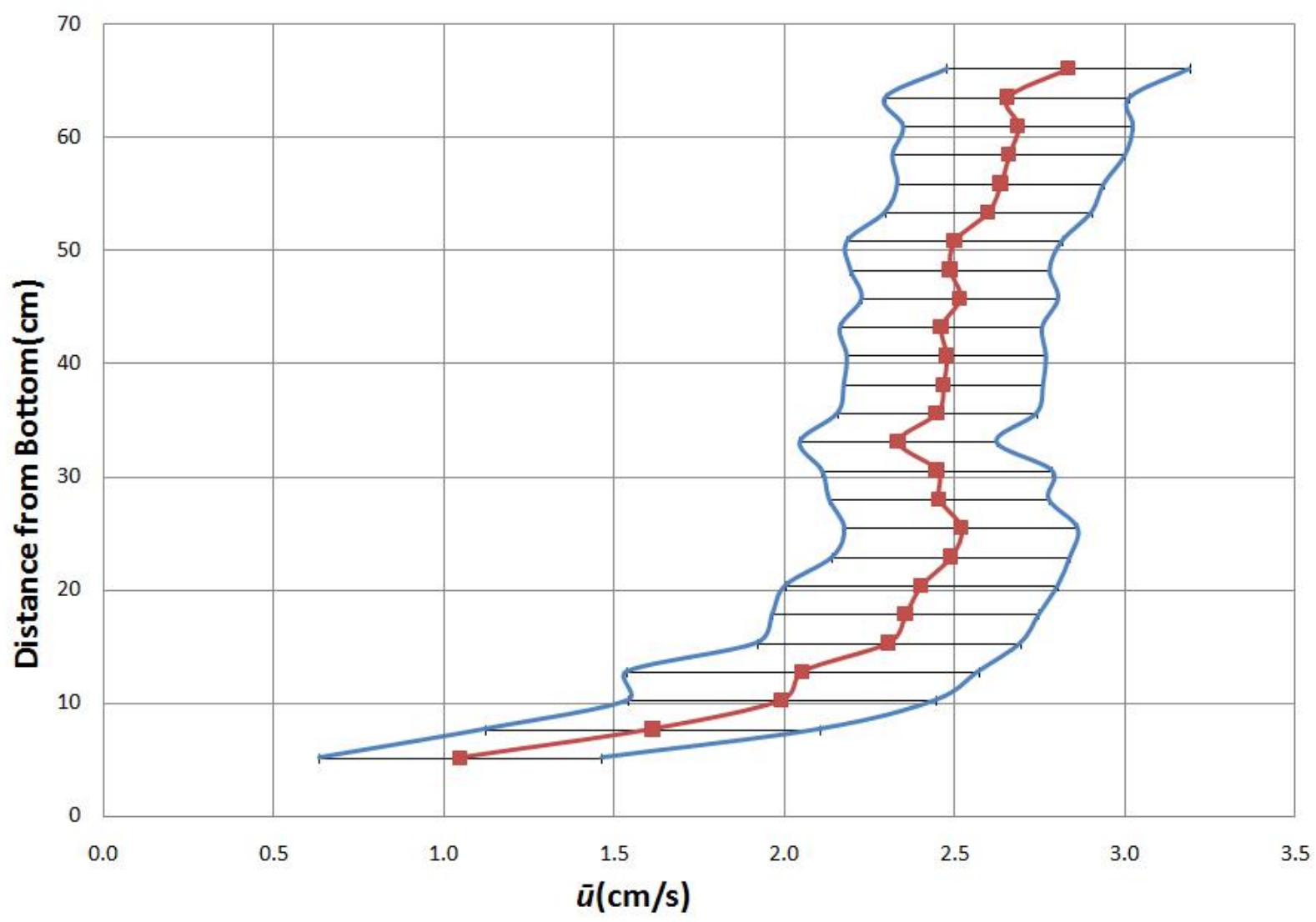

Figure 95: August 30 Vertical Profile of Mean Streamwise Velocity +/- One Standard Deviation 


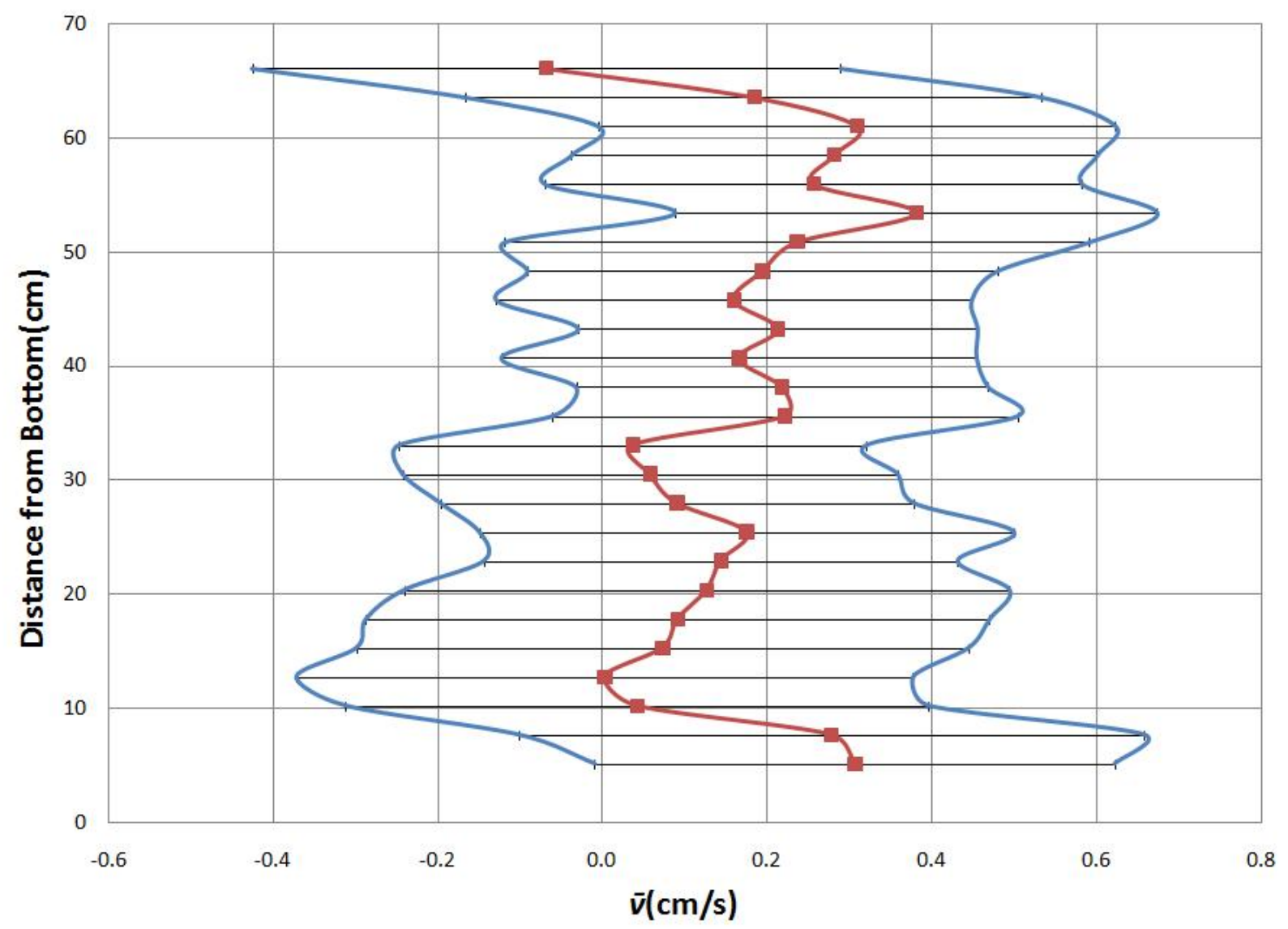

Figure 96: August 30 Vertical Profile of Mean Transverse Velocity +/- One Standard Deviation 


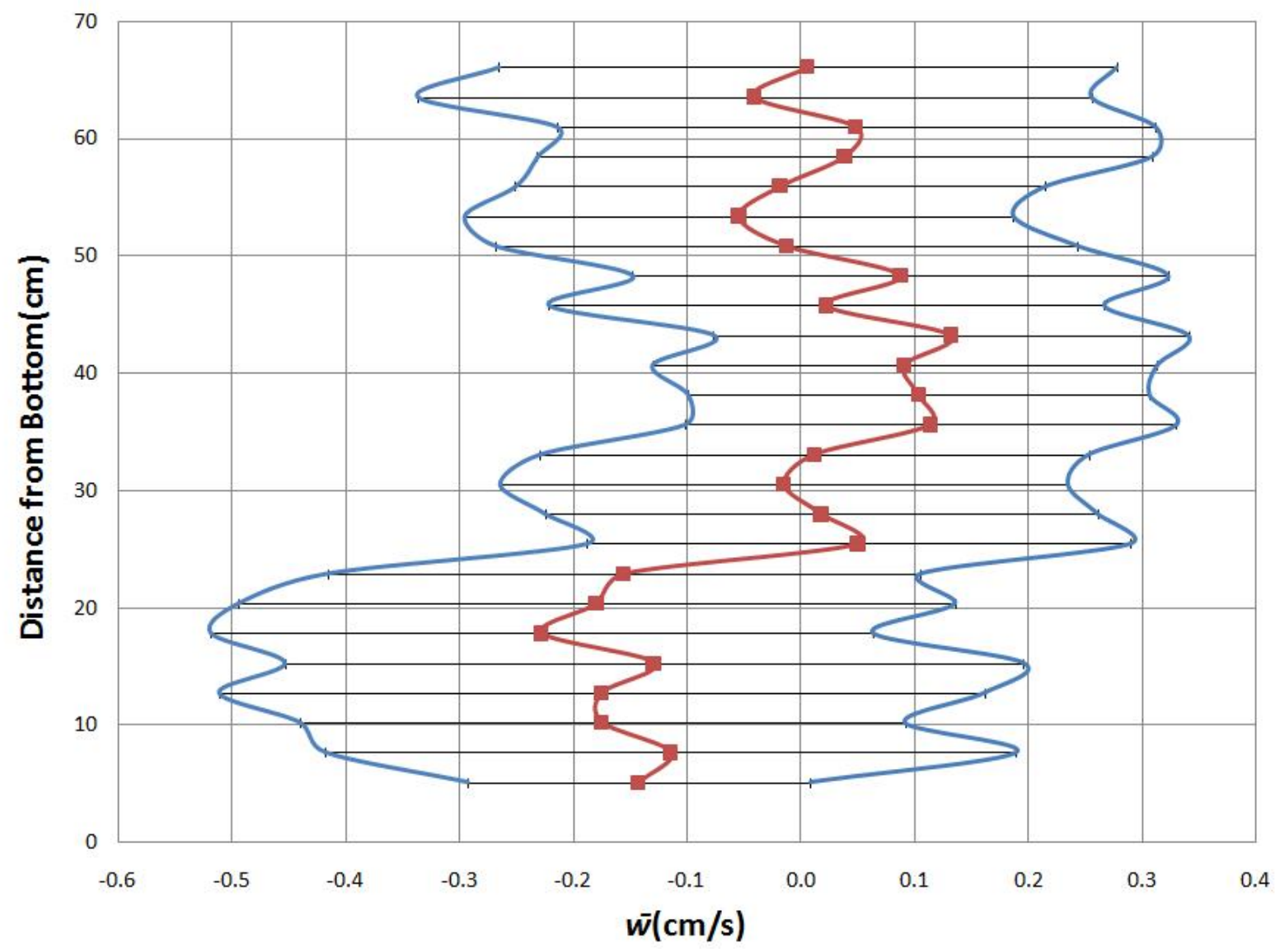

Figure 97: August 30 Vertical Profile of Mean Vertical Velocity +/- One Standard Deviation 


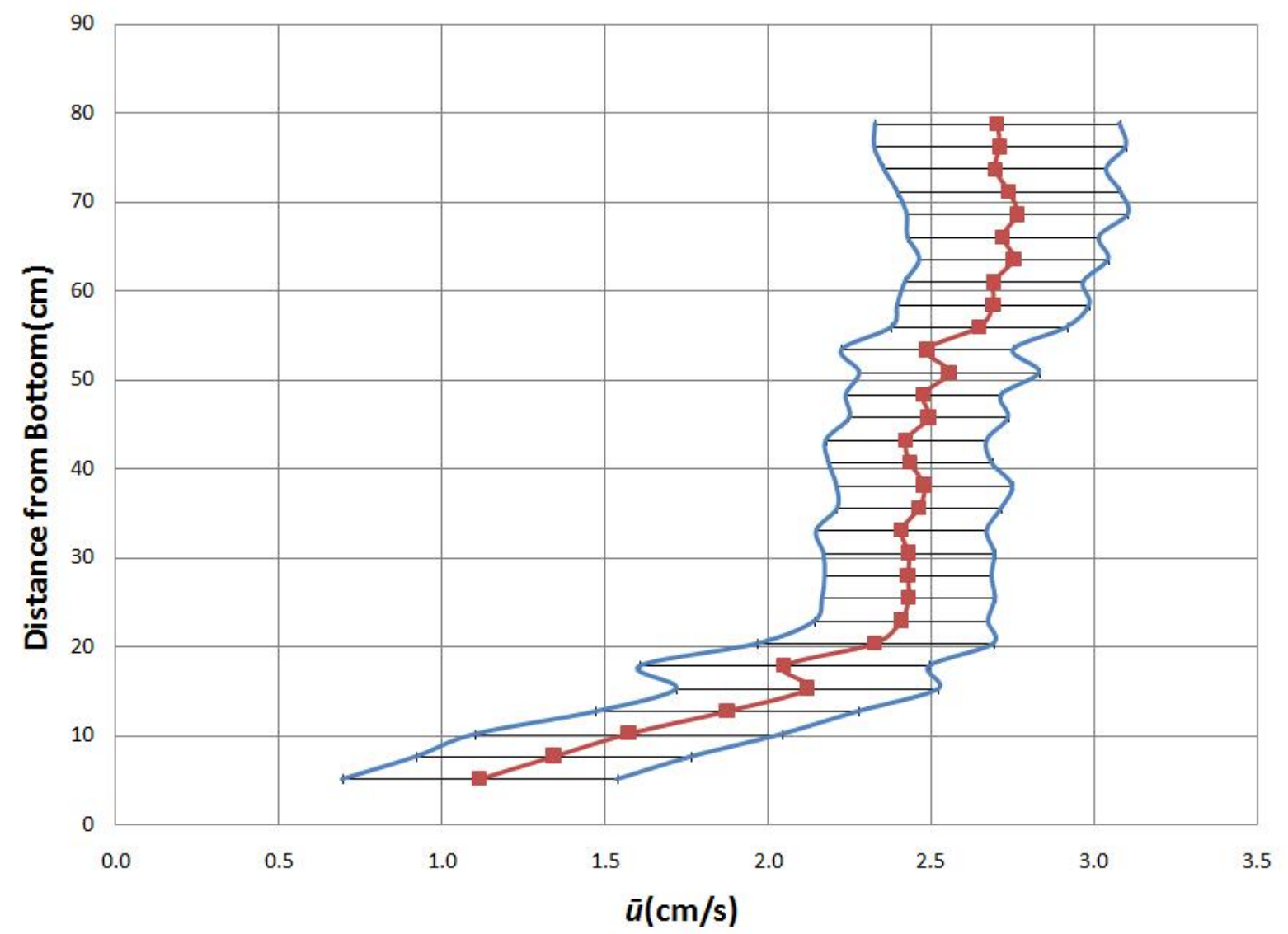

Figure 98: September 4 Vertical Profile of Mean Streamwise Velocity +/- One Standard Deviation 


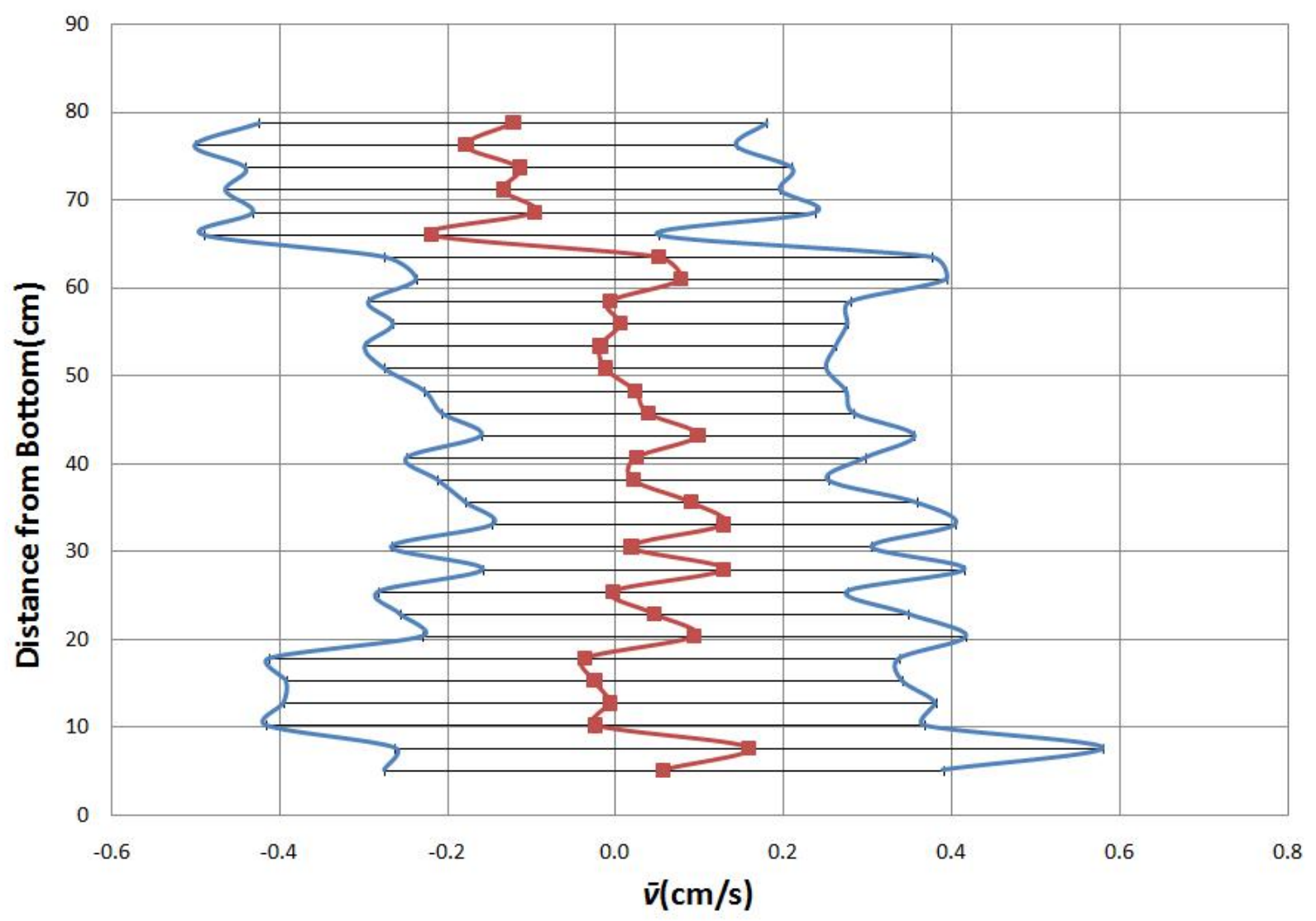

Figure 99: September 4 Vertical Profile of Mean Transverse Velocity +/- One Standard Deviation 


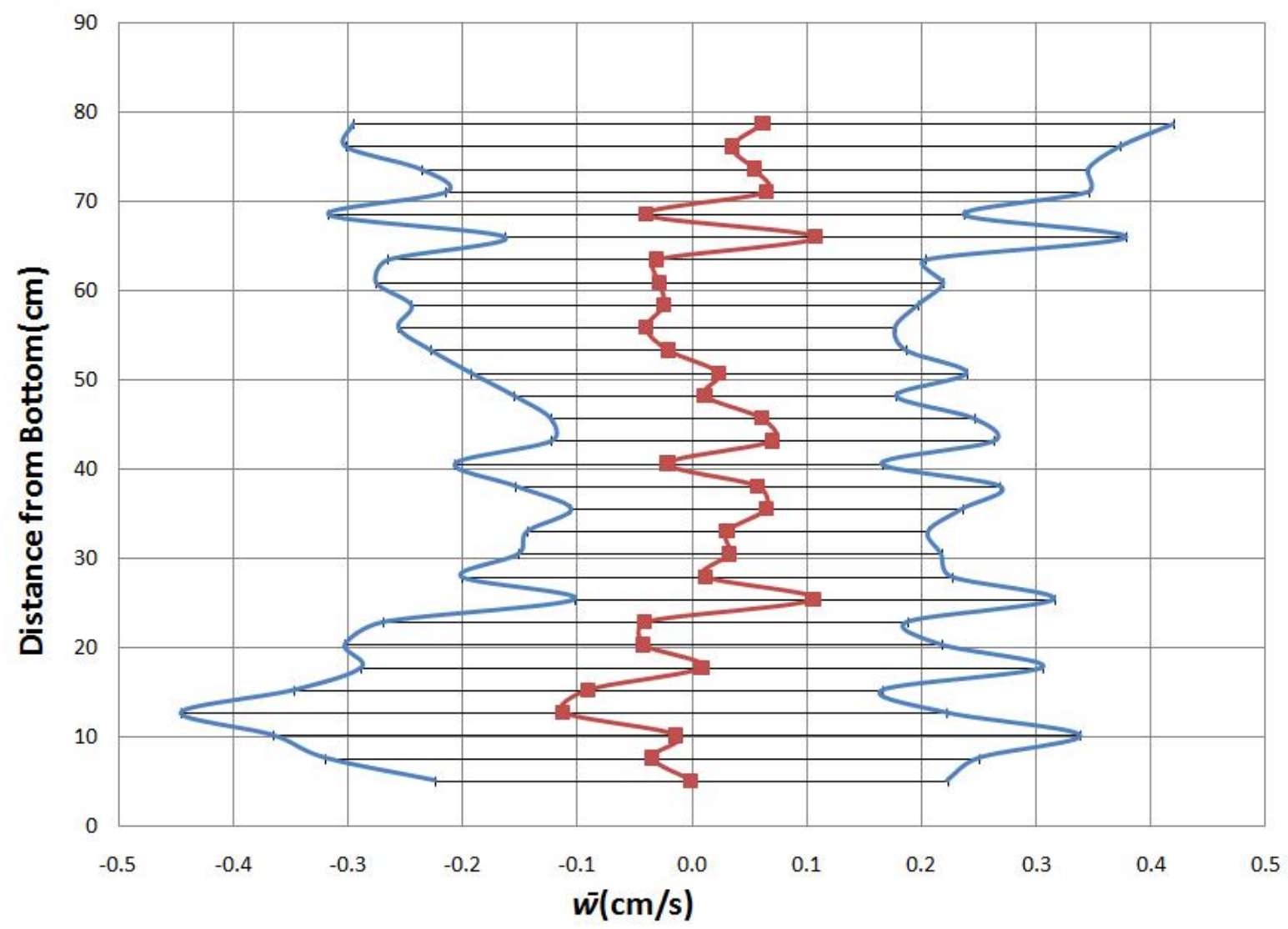

Figure 100: September 4 Vertical Profile of Mean Vertical Velocity +/- One Standard Deviation 


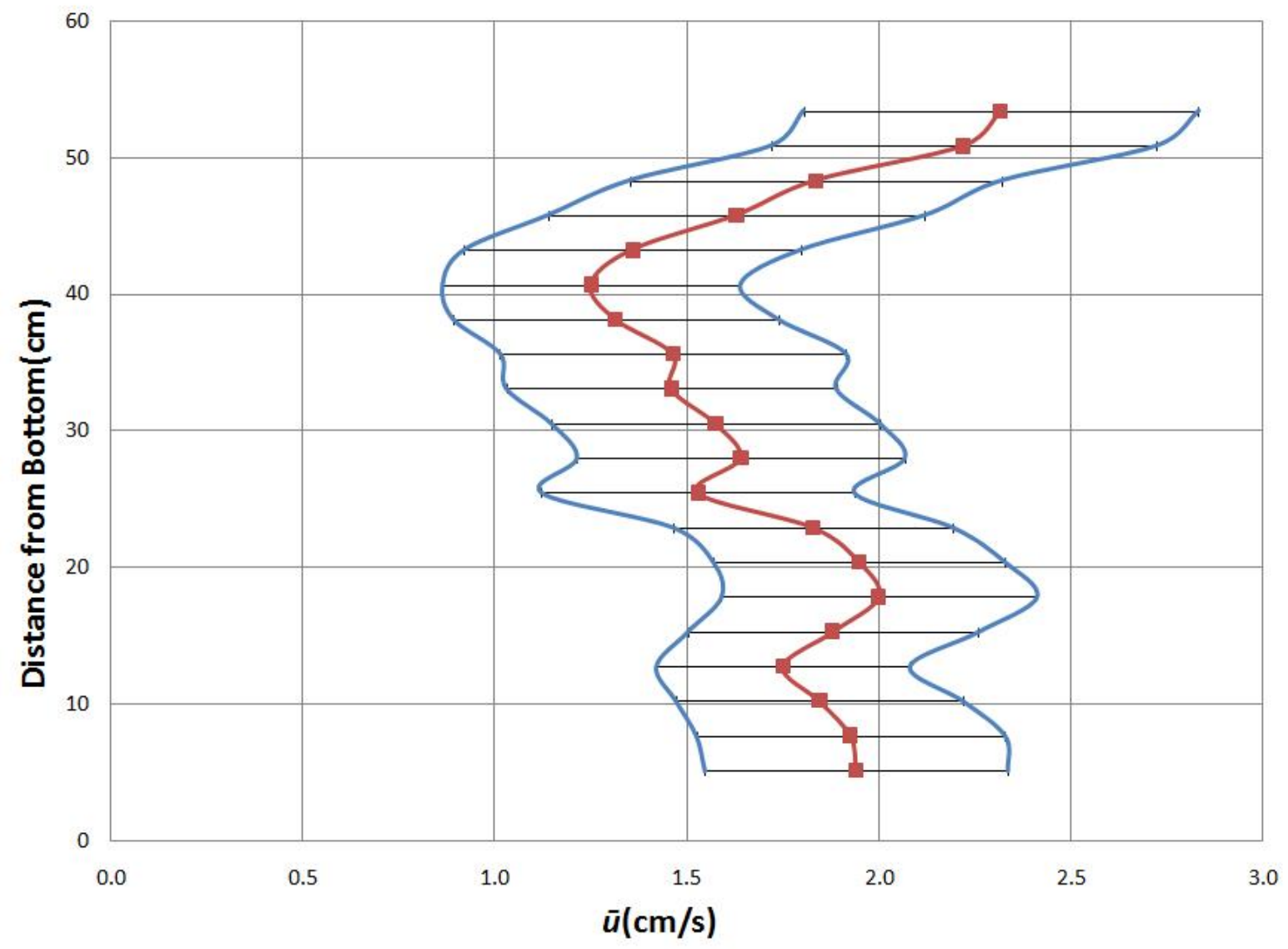

Figure 101: September 30 Vertical Profile of Mean Streamwise Velocity +/- One Standard Deviation 


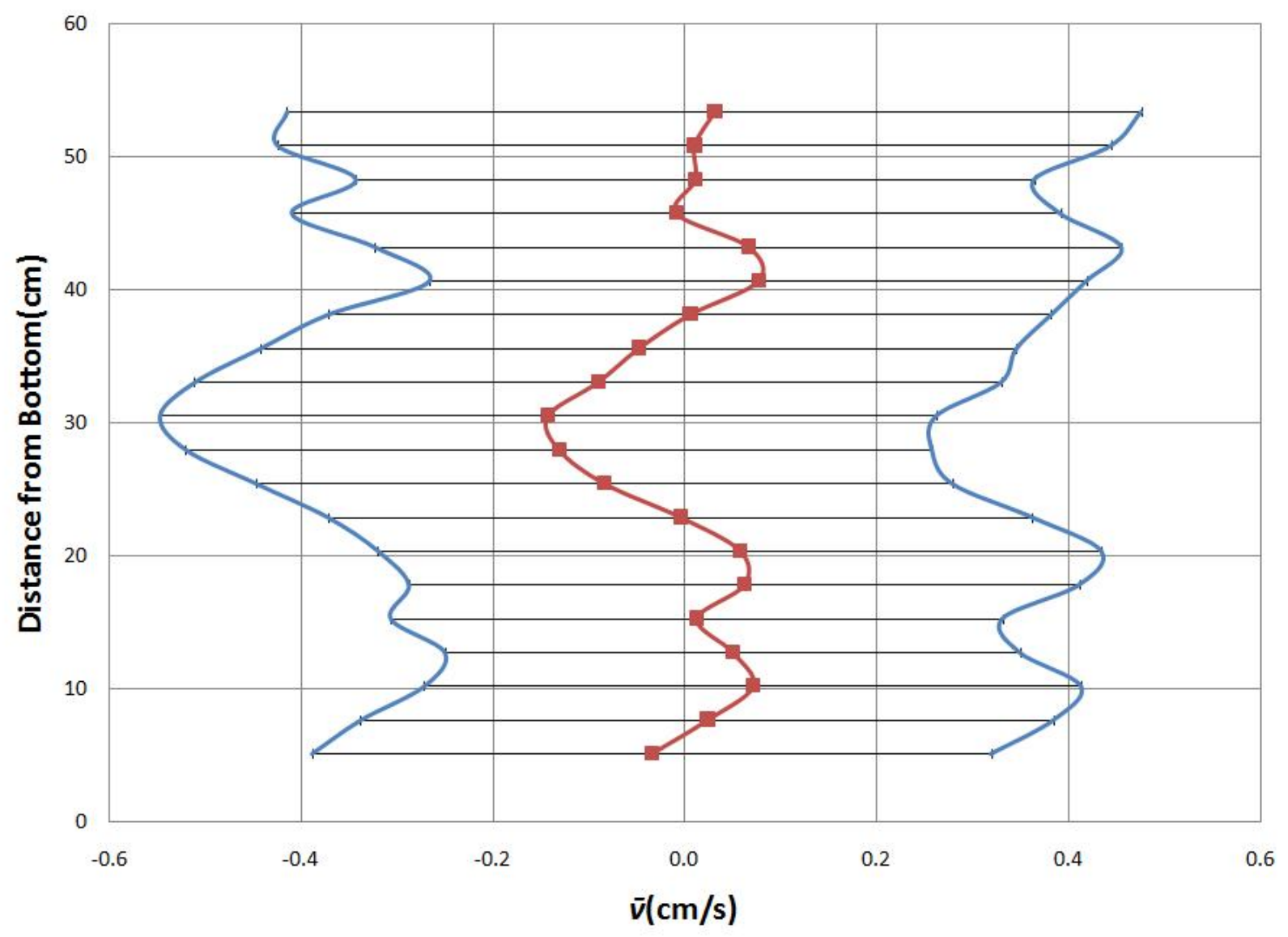

Figure 102: September 30 Vertical Profile of Mean Transverse Velocity +/- One Standard Deviation 


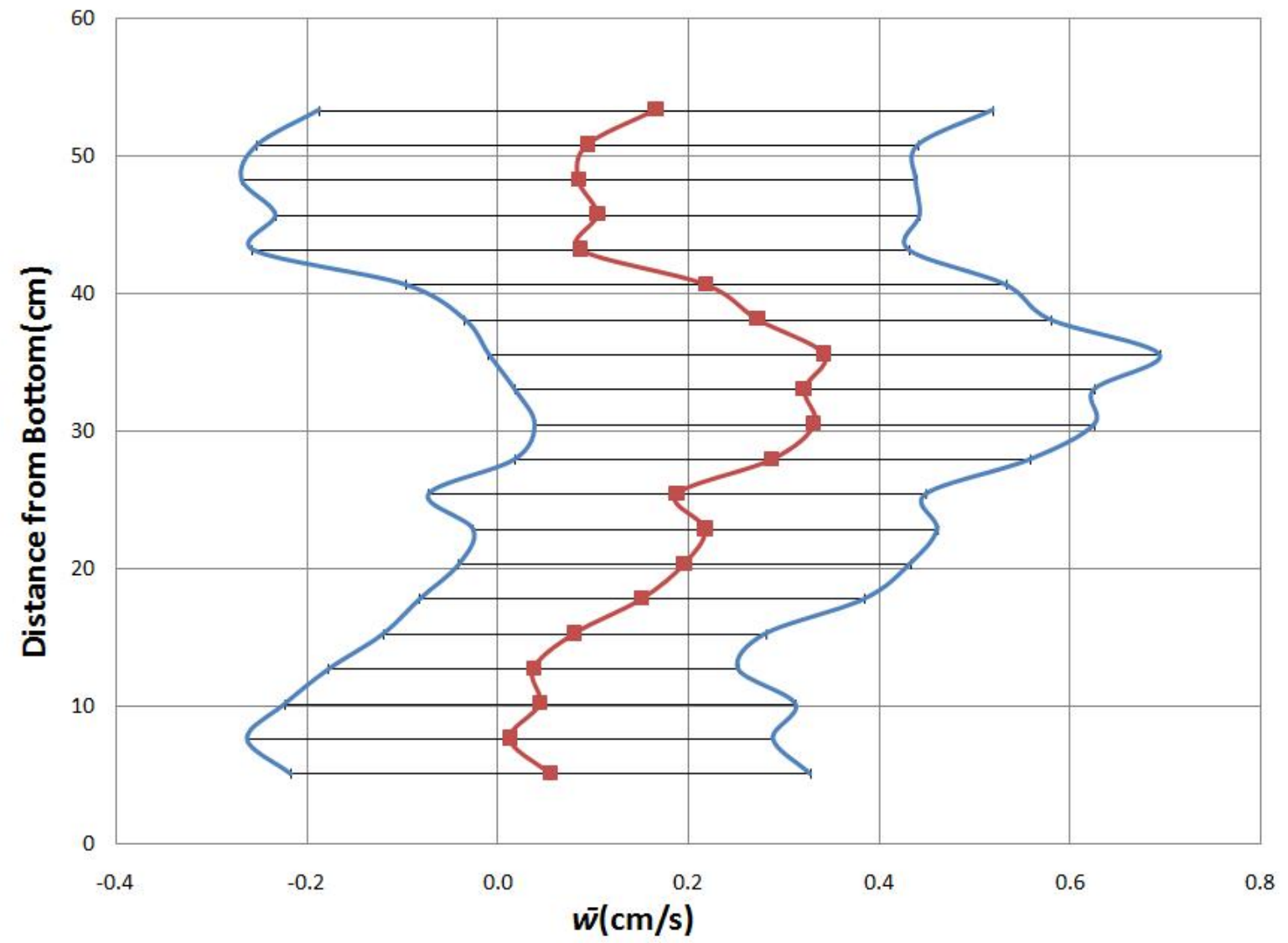

Figure 103: September 30 Vertical Profile of Mean Vertical Velocity +/- One Standard Deviation 


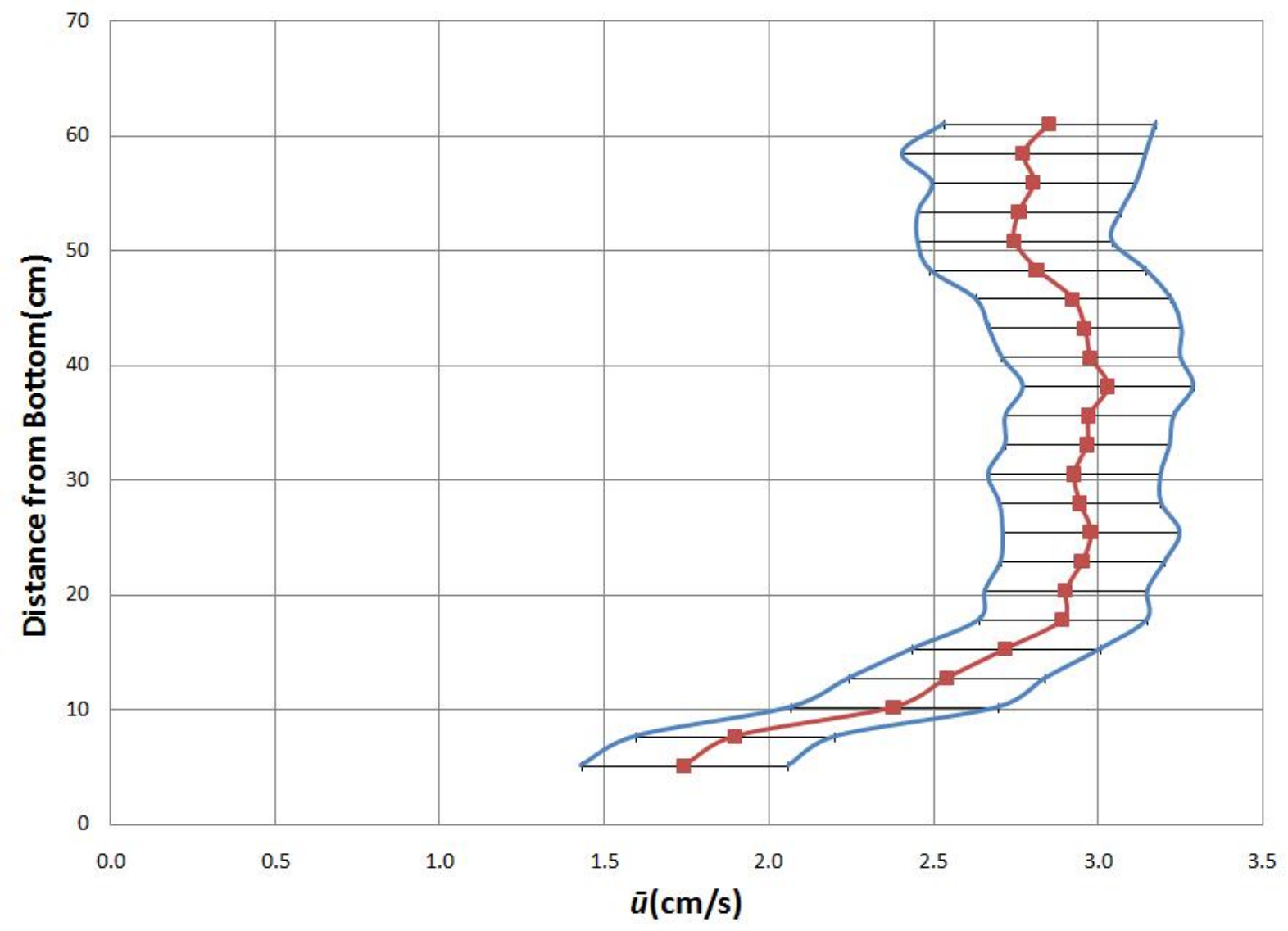

Figure 104: October 9 Vertical Profile of Mean Streamwise Velocity +/- One Standard Deviation 


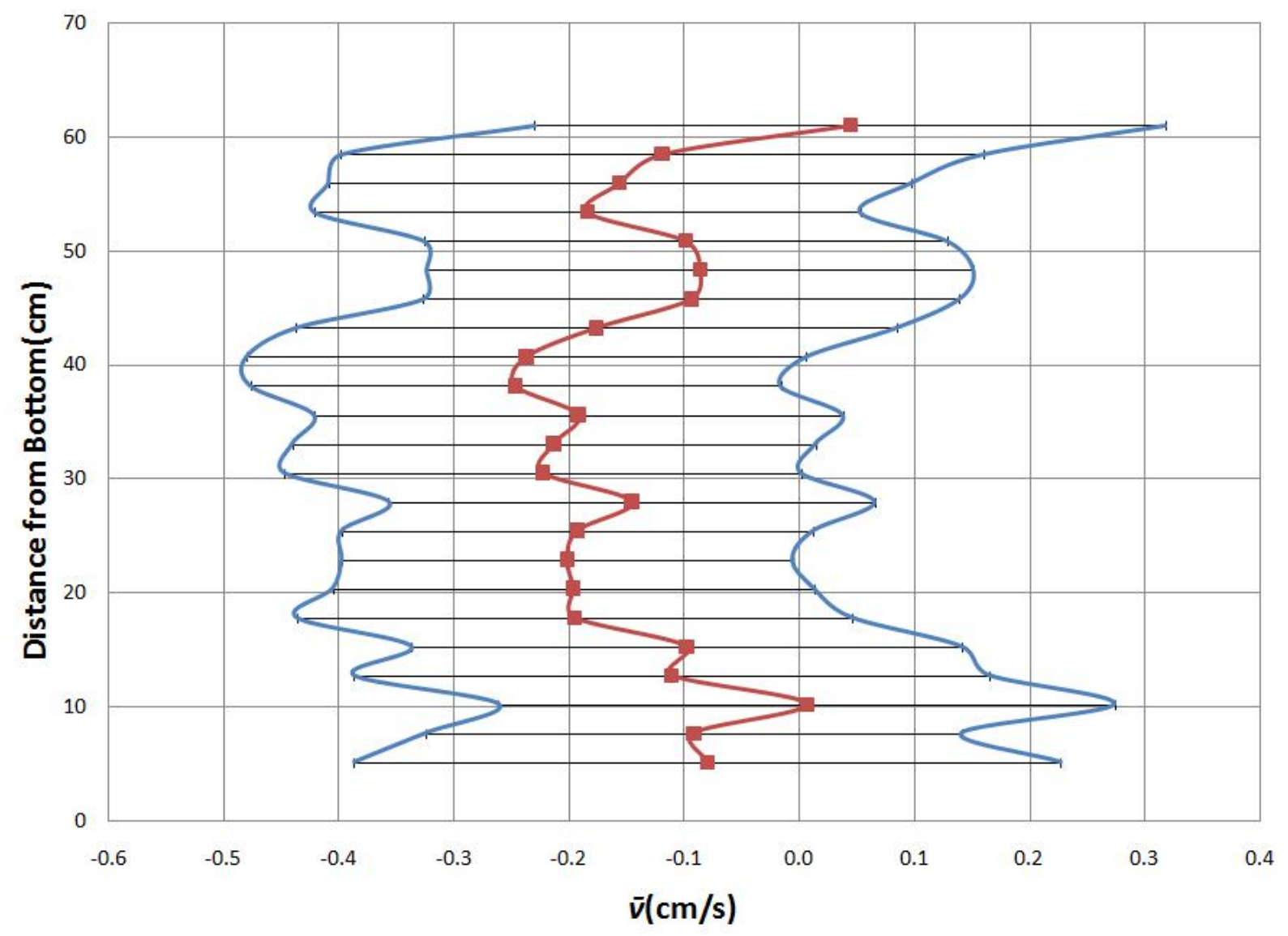

Figure 105: October 9 Vertical Profile of Mean Transverse Velocity +/- One Standard Deviation 


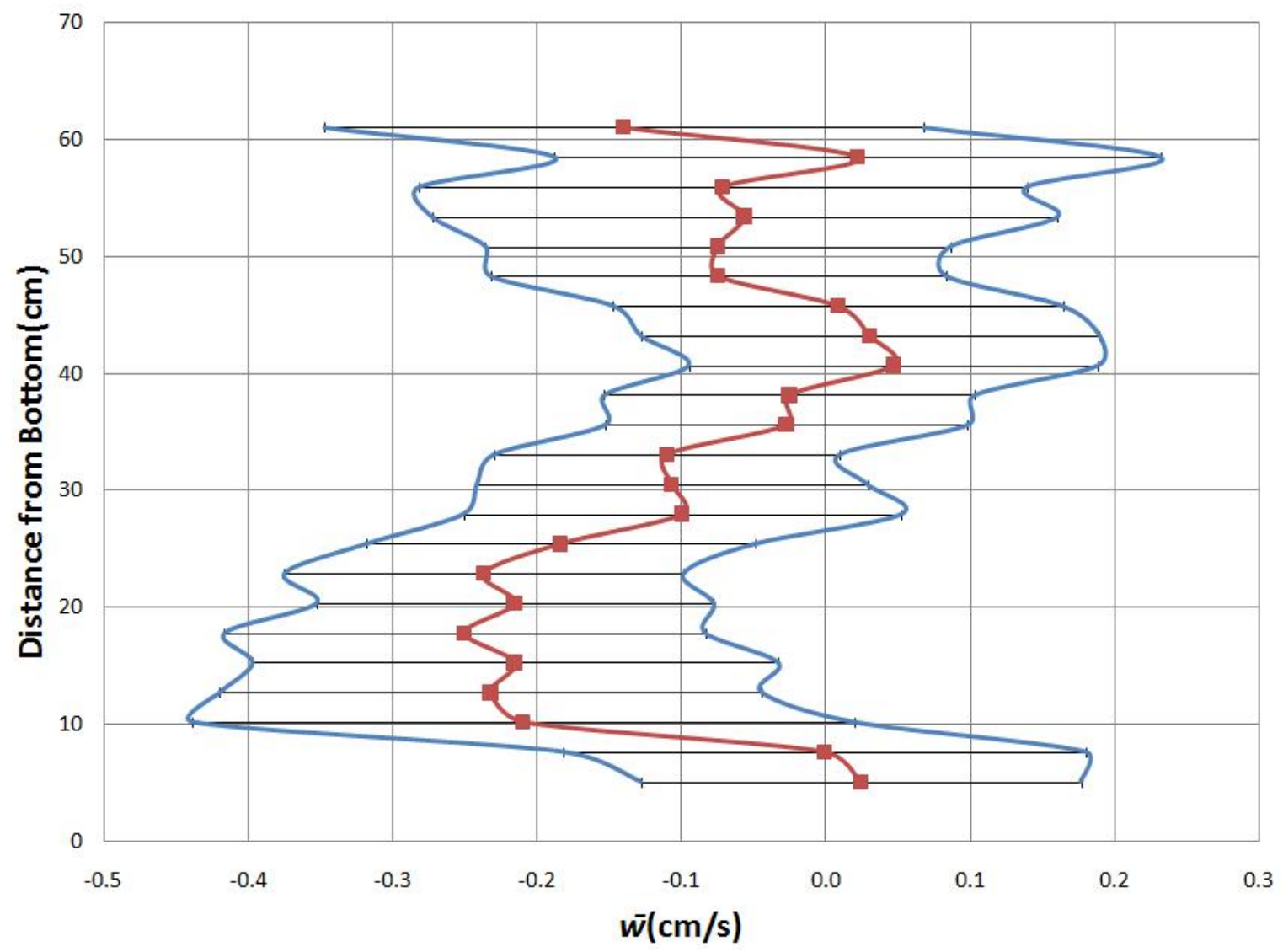

Figure 106: October 9 Vertical Profile of Mean Vertical Velocity +/- One Standard Deviation 


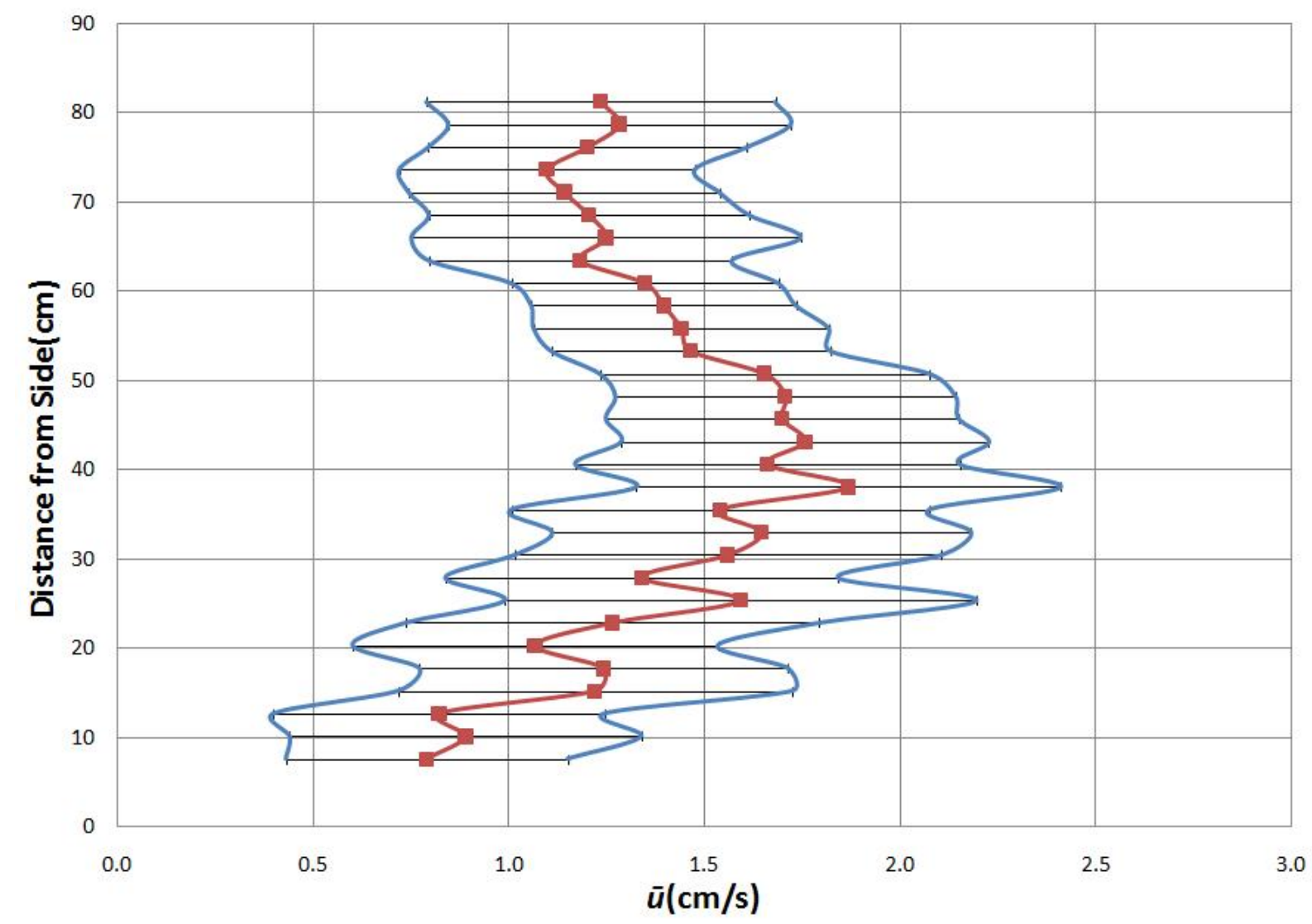

Figure 107: November 4 Transverse Profile of Mean Streamwise Velocity +/- One Standard Deviation 


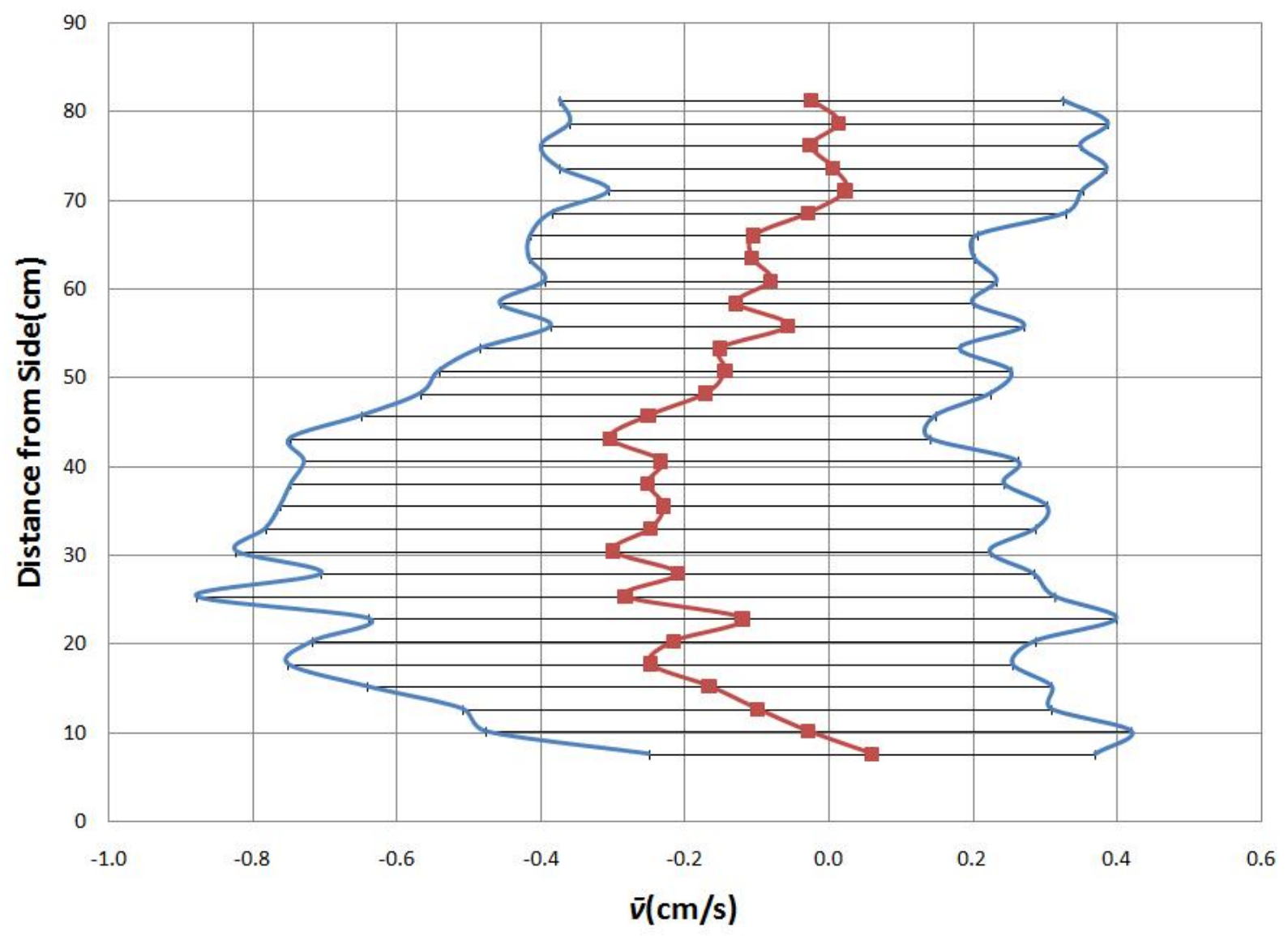

Figure 108: November 4 Transverse Profile of Mean Transverse Velocity +/- One Standard Deviation 


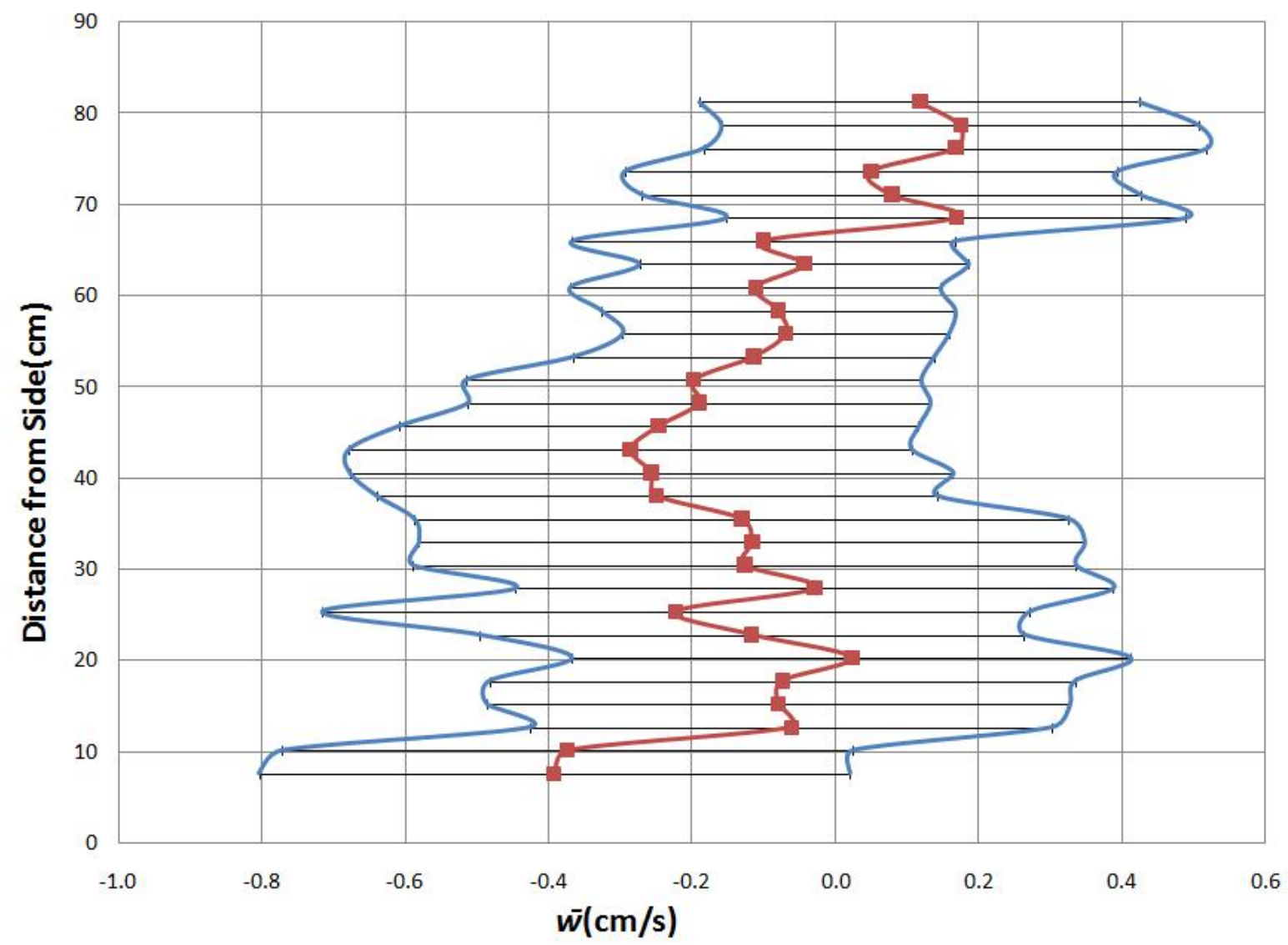

Figure 109: November 4 Transverse Profile of Mean Vertical Velocity +/- One Standard Deviation 


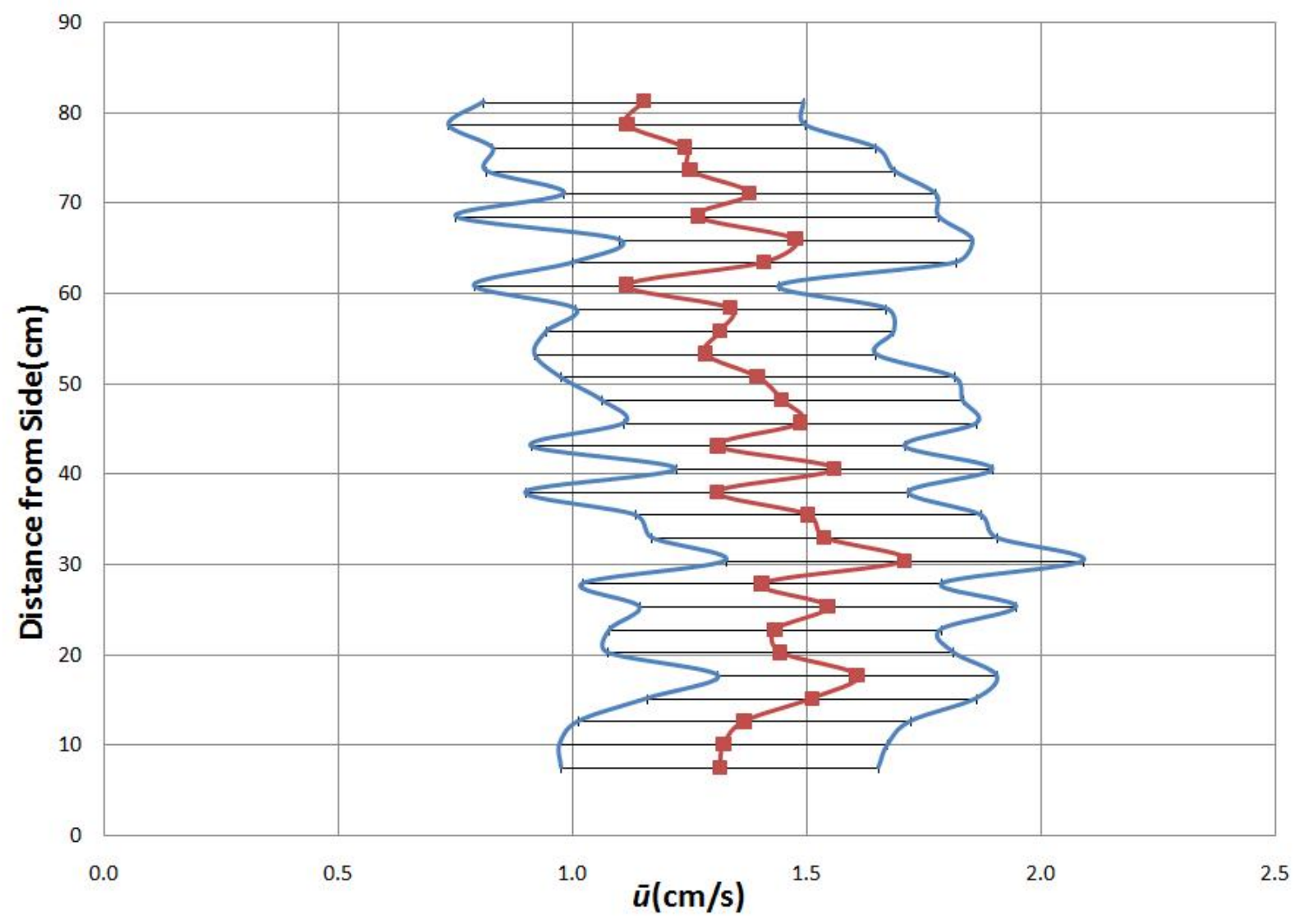

Figure 110: November 6 Transverse Profile of Mean Streamwise Velocity +/- One Standard Deviation 


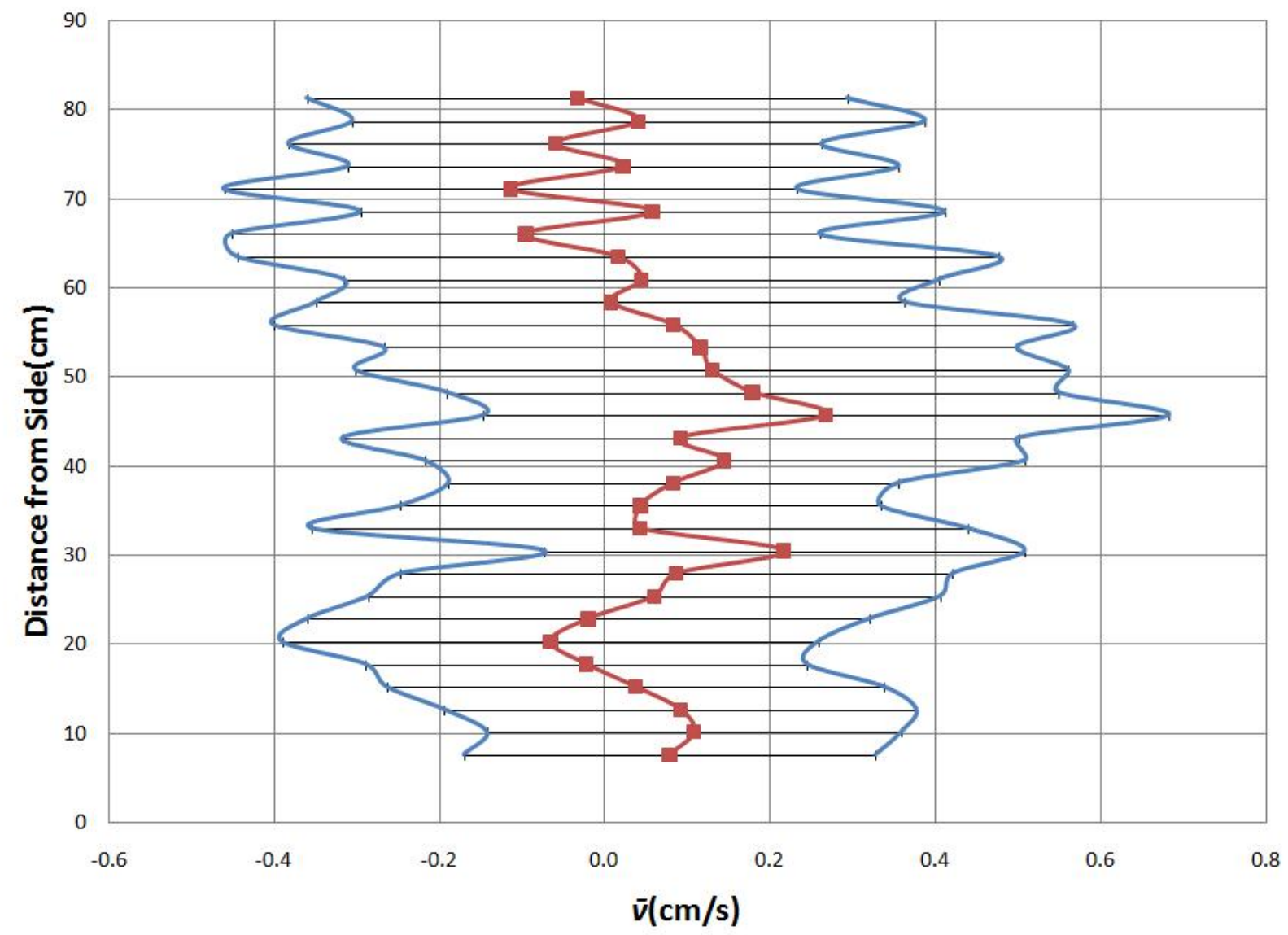

Figure 111: November 6 Transverse Profile of Mean Transverse Velocity +/- One Standard Deviation 


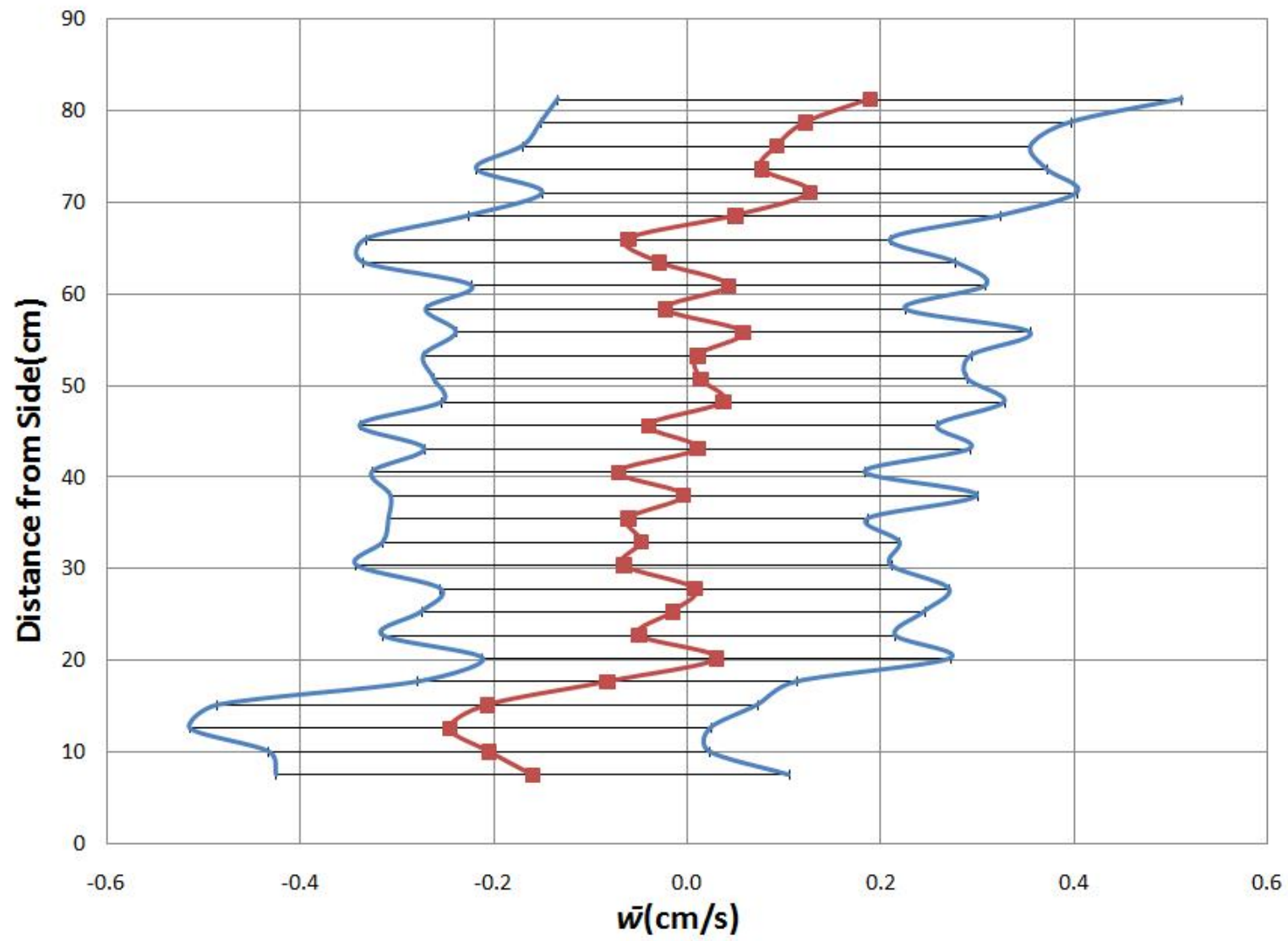

Figure 112: November 6 Transverse Profile of Mean Vertical Velocity +/- One Standard Deviation 


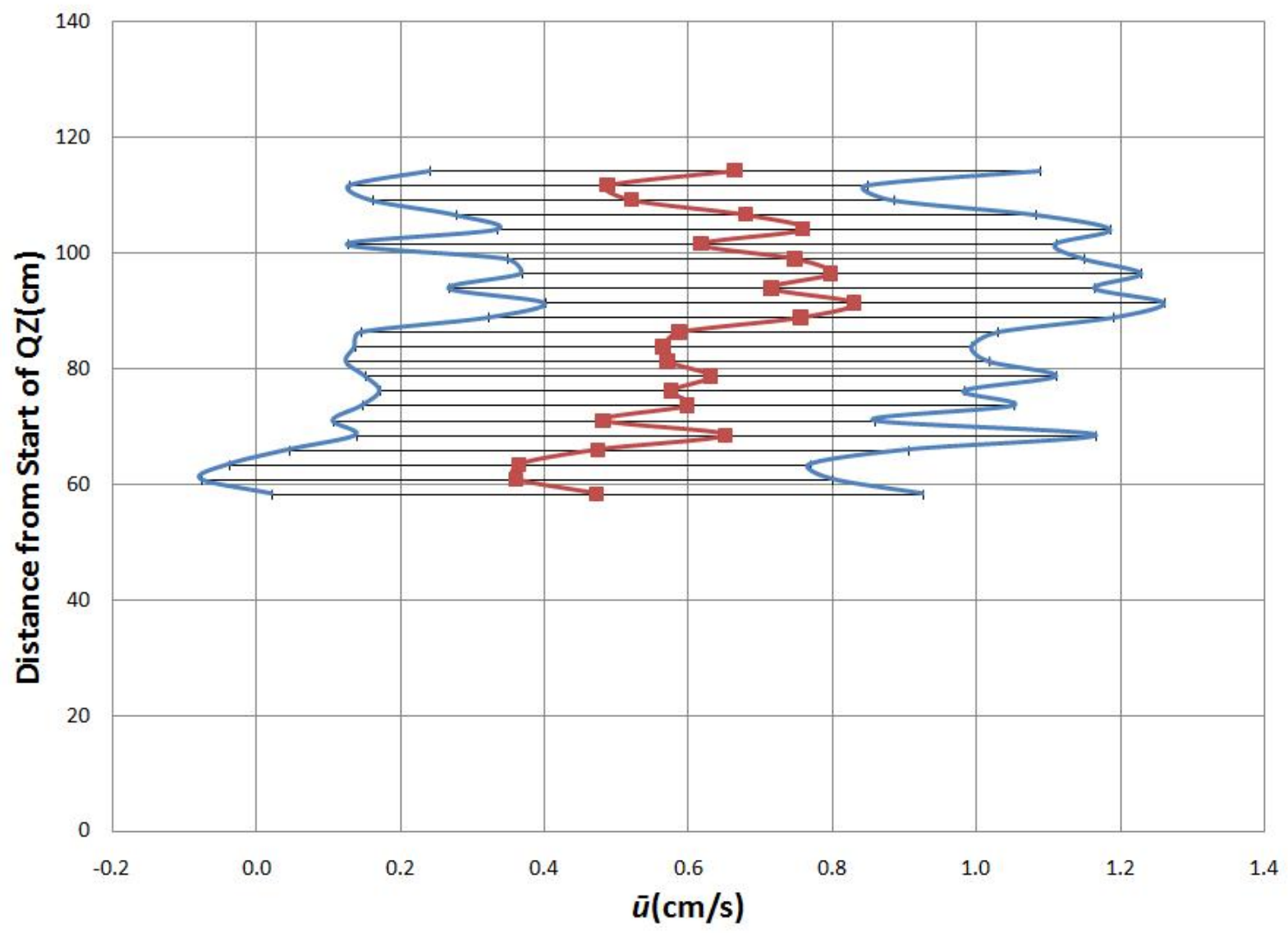

Figure 113: November 26 Streamwise Profile of Mean Streamwise Velocity +/- One Standard Deviation 


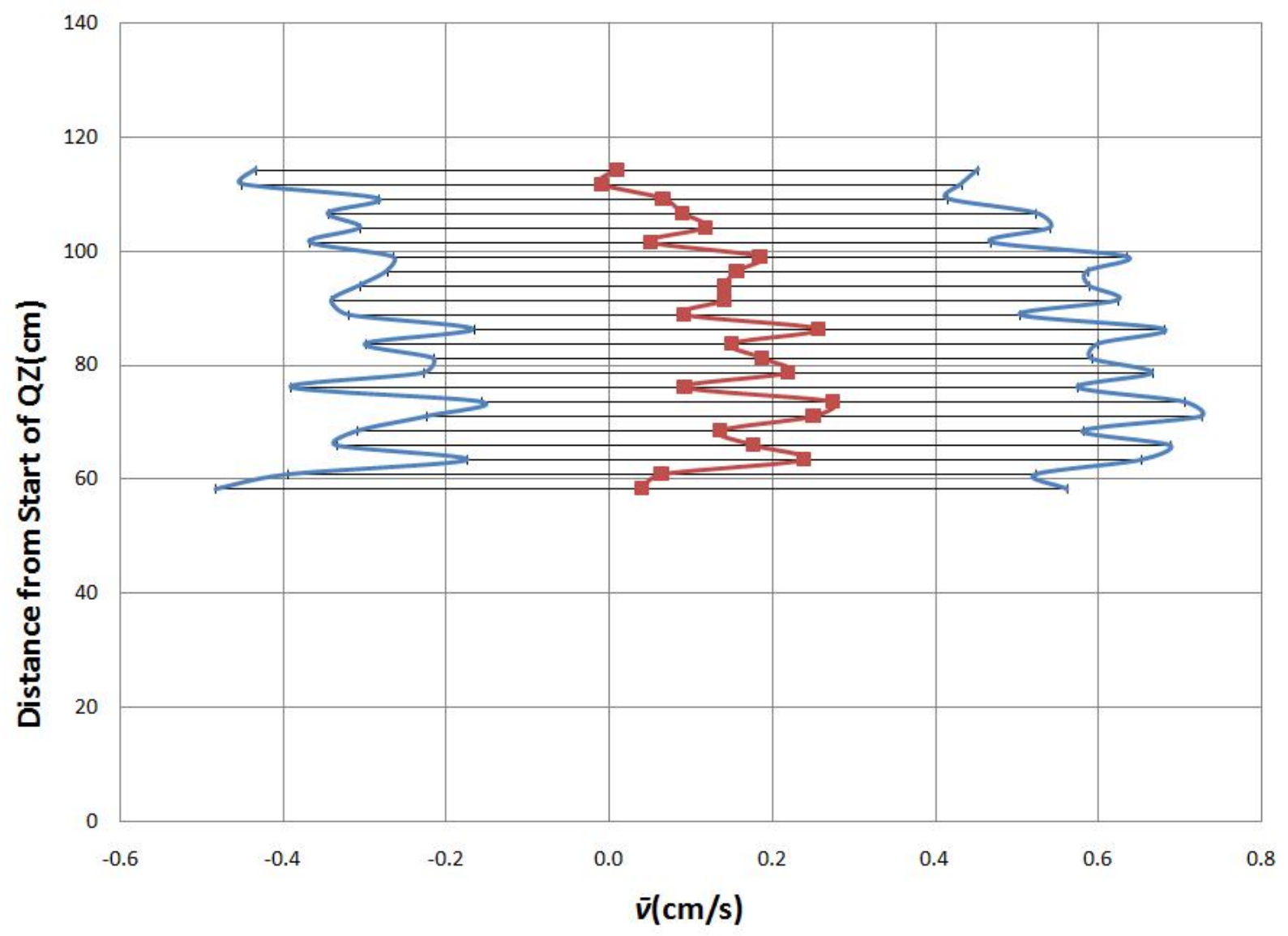

Figure 114: November 26 Streamwise Profile of Mean Transverse Velocity +/- One Standard Deviation 


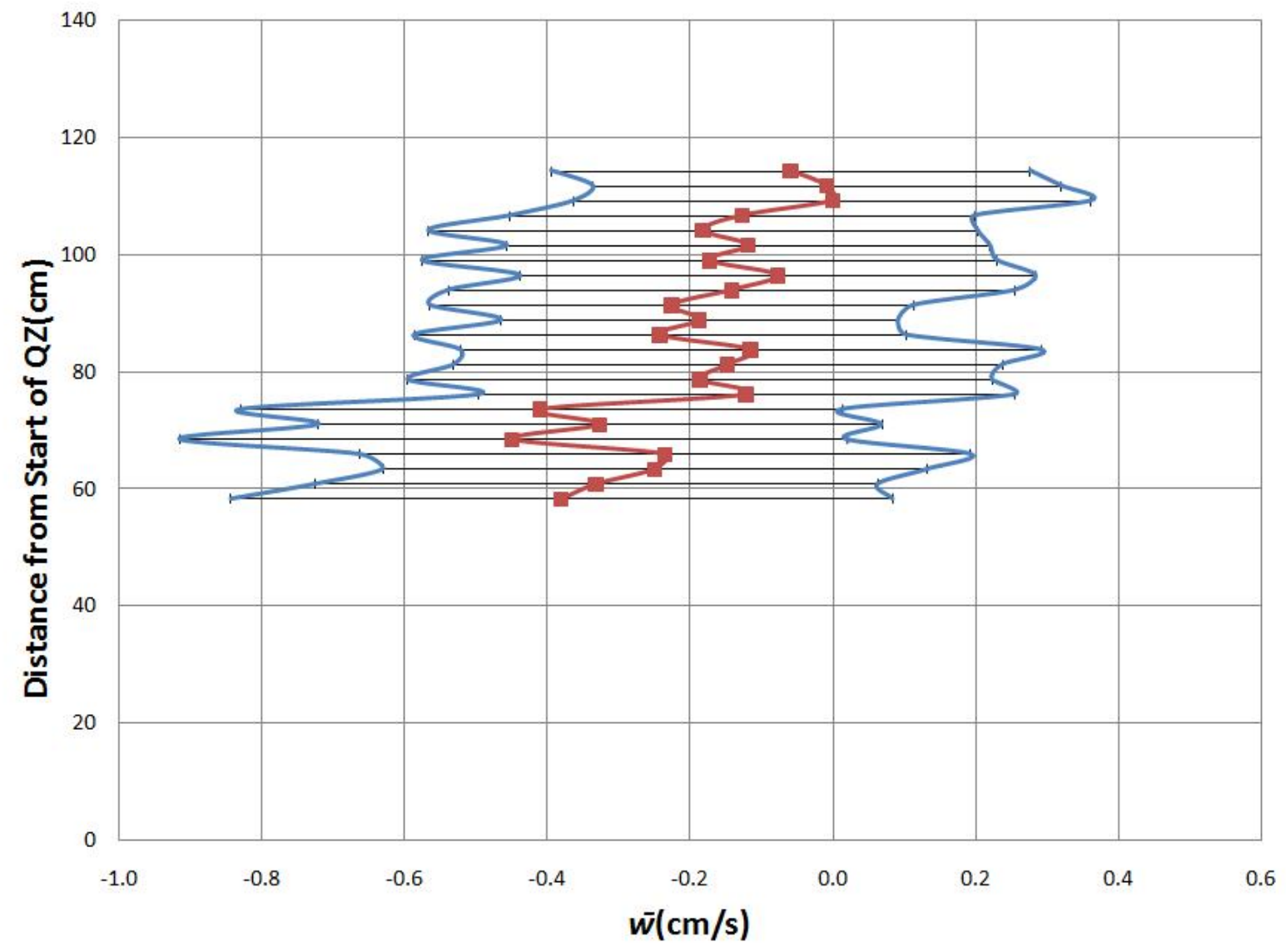

Figure 115: November 26 Streamwise Profile of Mean Vertical Velocity +/- One Standard Deviation 


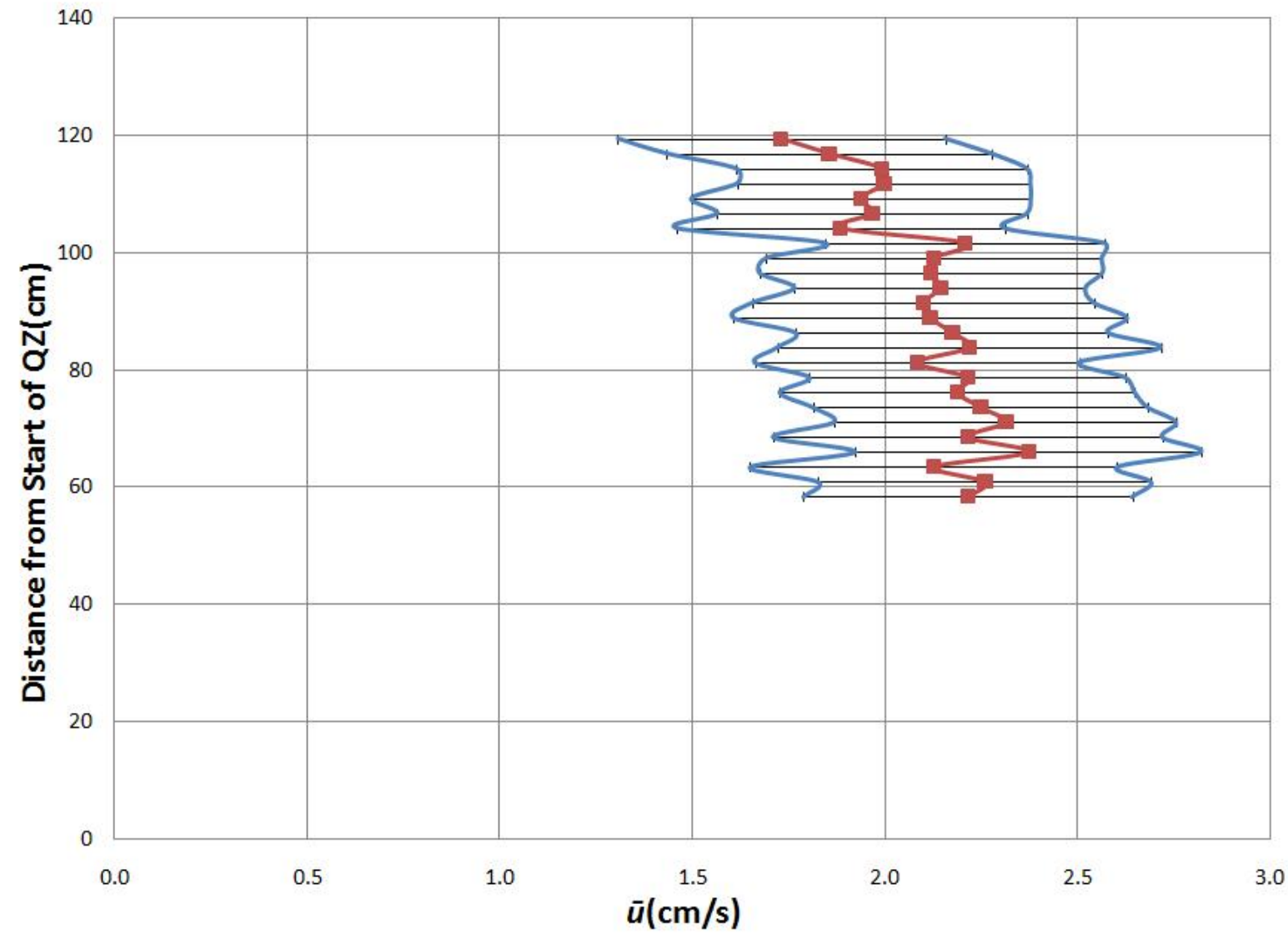

Figure 116: November 29 Streamwise Profile of Mean Streamwise Velocity +/- One Standard Deviation 


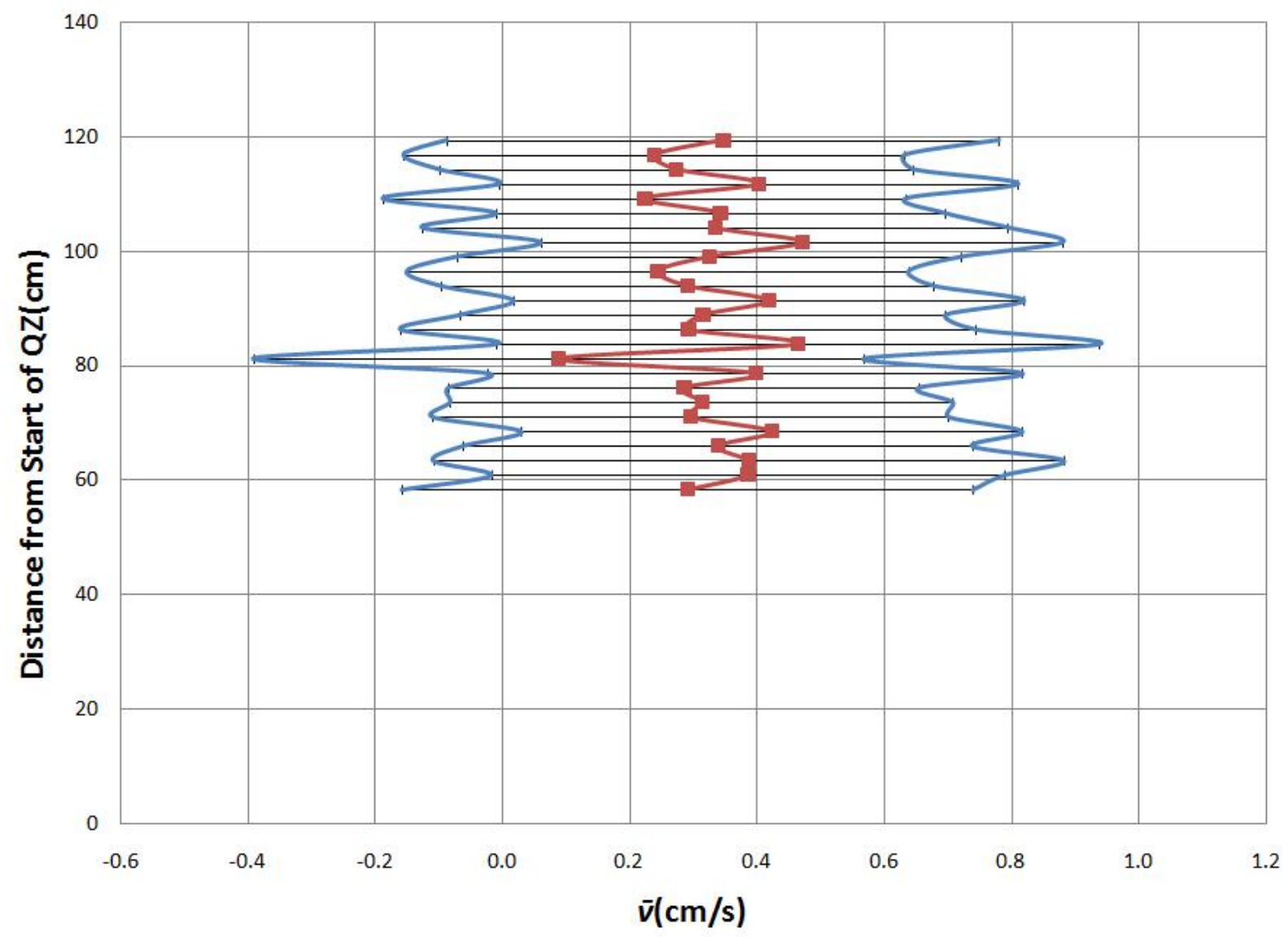

Figure 117: November 29 Streamwise Profile of Mean Transverse Velocity +/- One Standard Deviation 


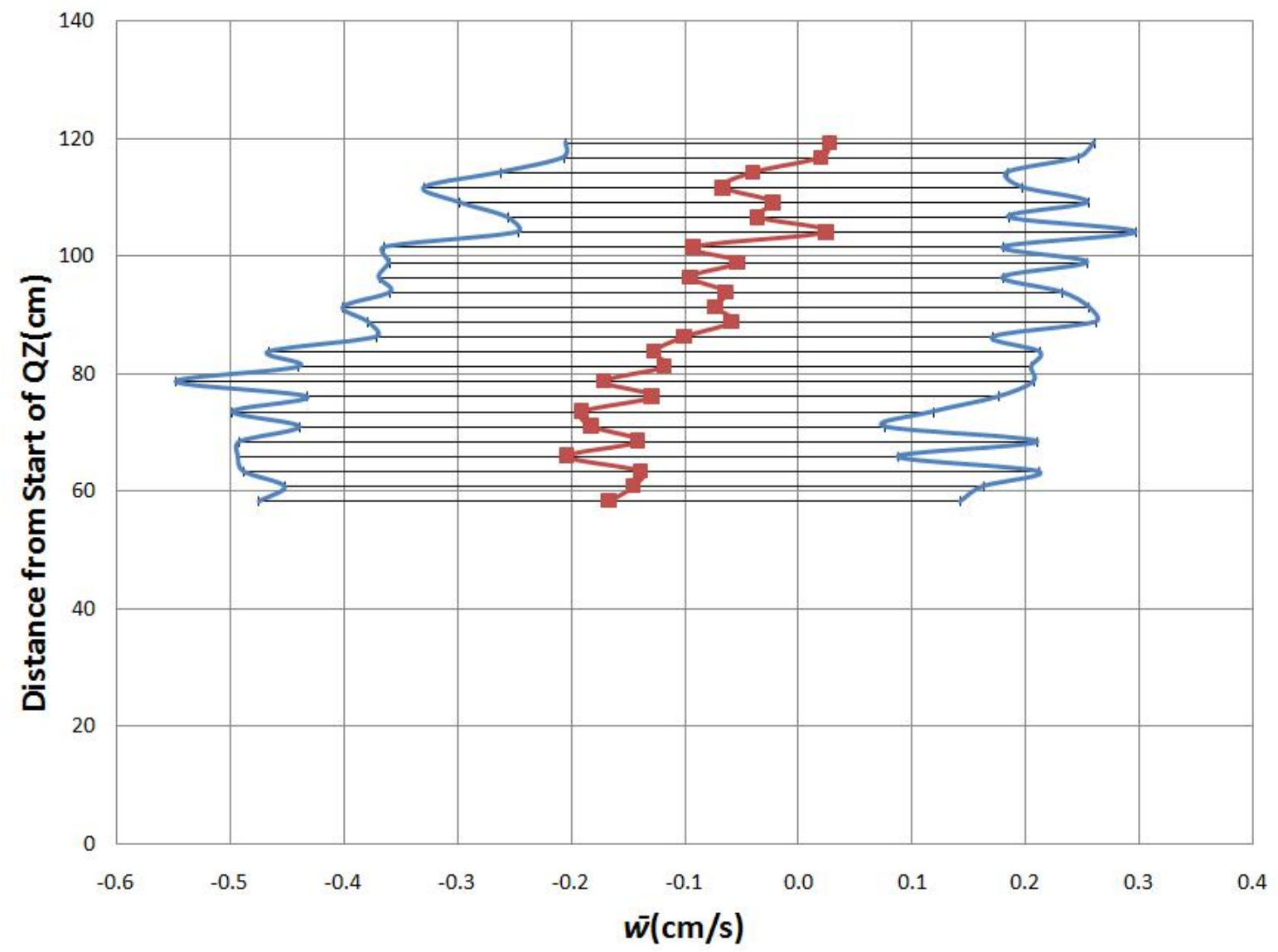

Figure 118: November 29 Streamwise Profile of Mean Vertical Velocity +/- One Standard Deviation 


\section{Appendix C: Additional Profiles}

This appendix contains all mean velocity profiles measured with error bars representing the standard deviation.

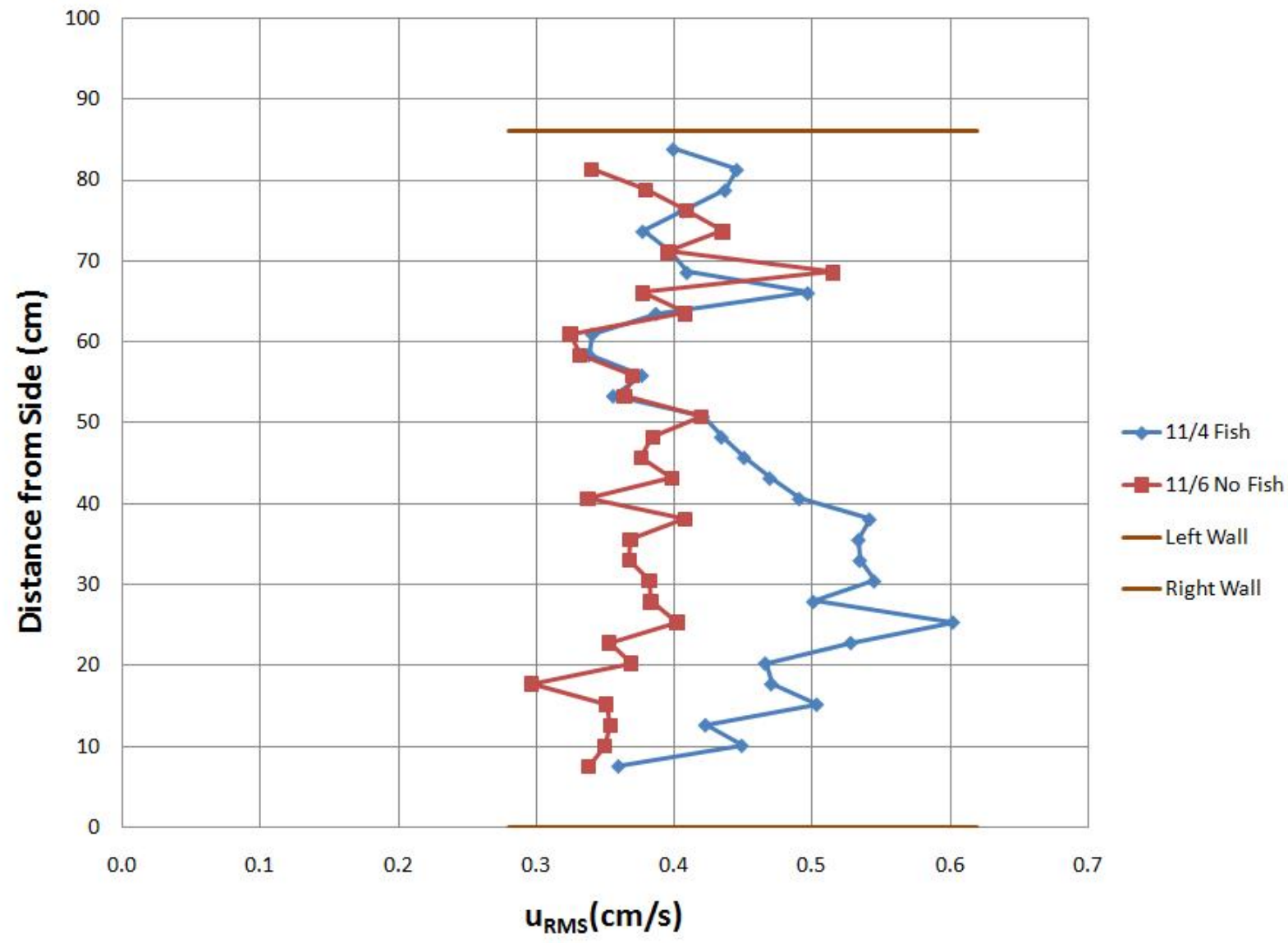

Figure 119: Transverse Profiles of Root Mean Square Streamwise Velocity 


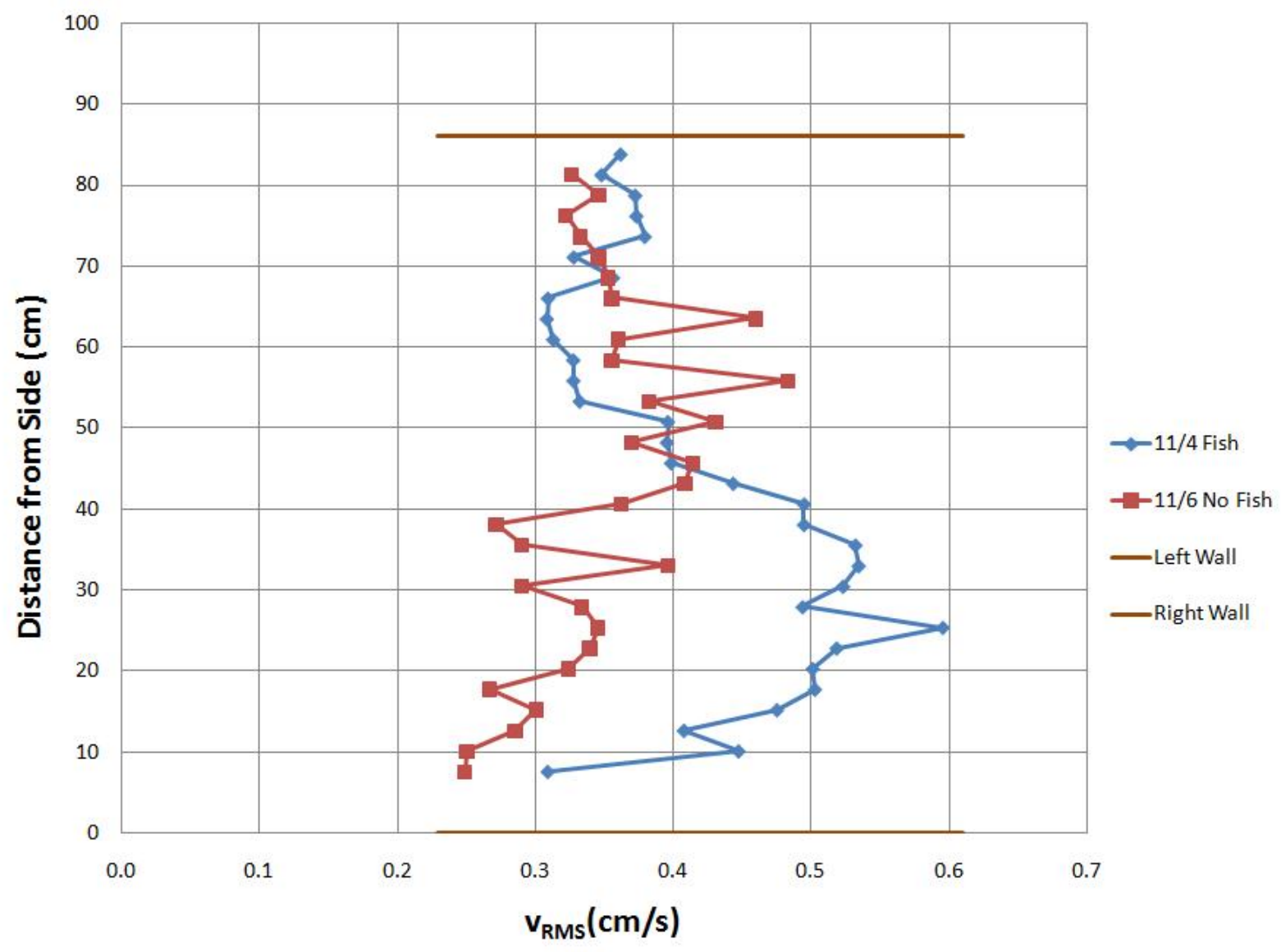

Figure 120: Transverse Profiles of Root Mean Square Transverse Velocity 


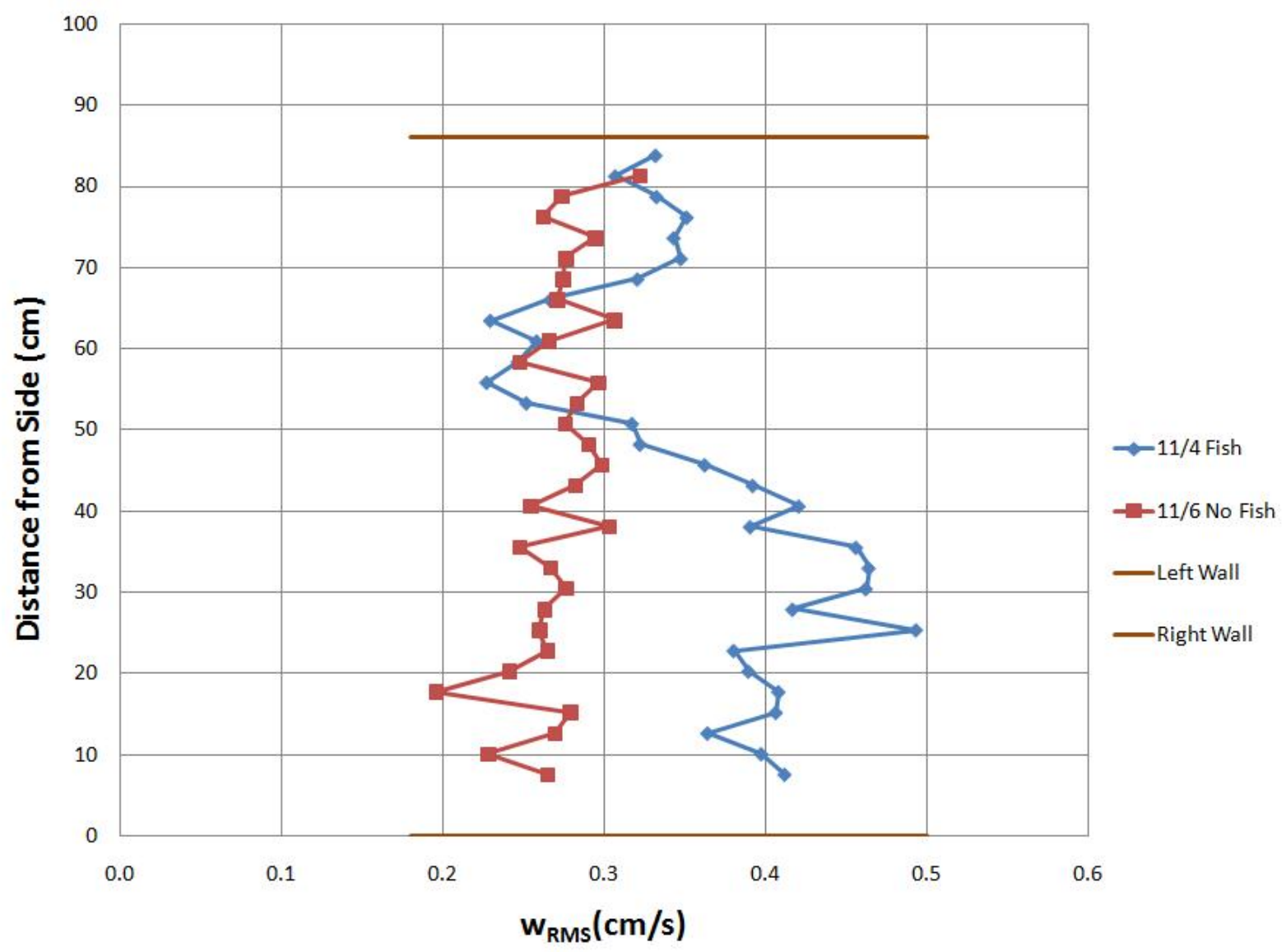

Figure 121: Transverse Profiles of Root Mean Square Vertical Velocity 


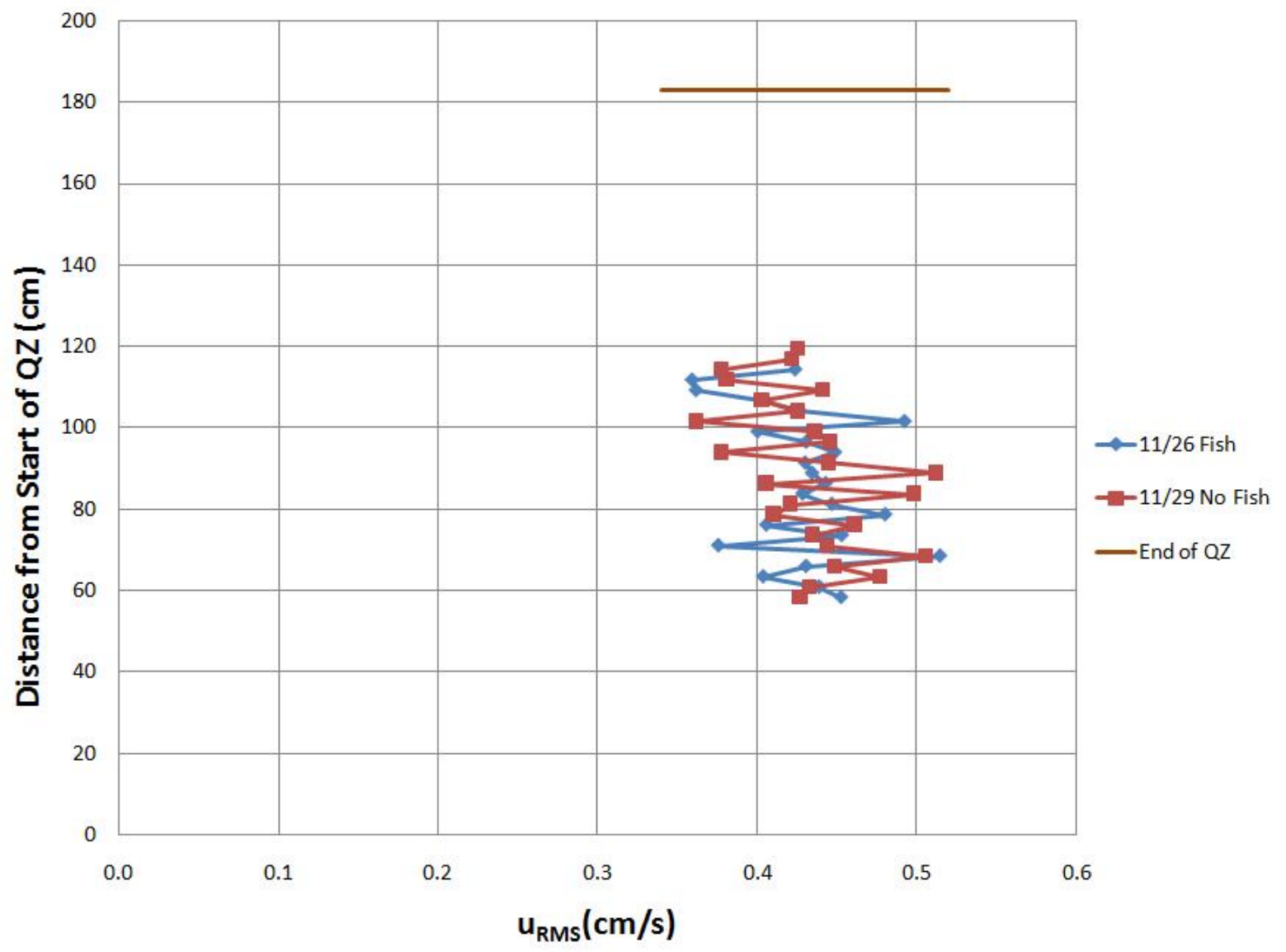

Figure 122: Streamwise Profiles of Root Mean Square Streamwise Velocity 


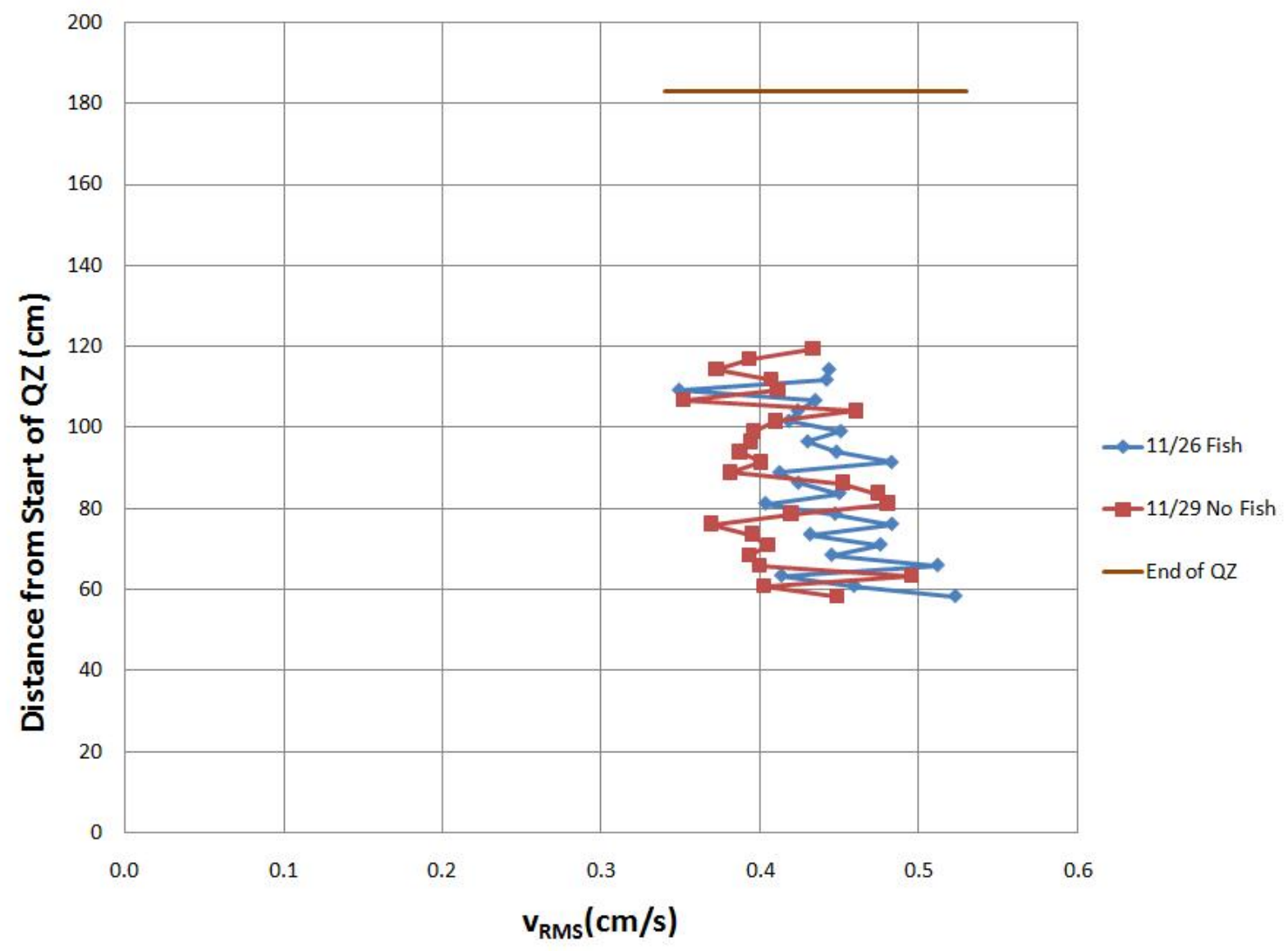

Figure 123: Streamwise Profiles of Root Mean Square Transverse Velocity 


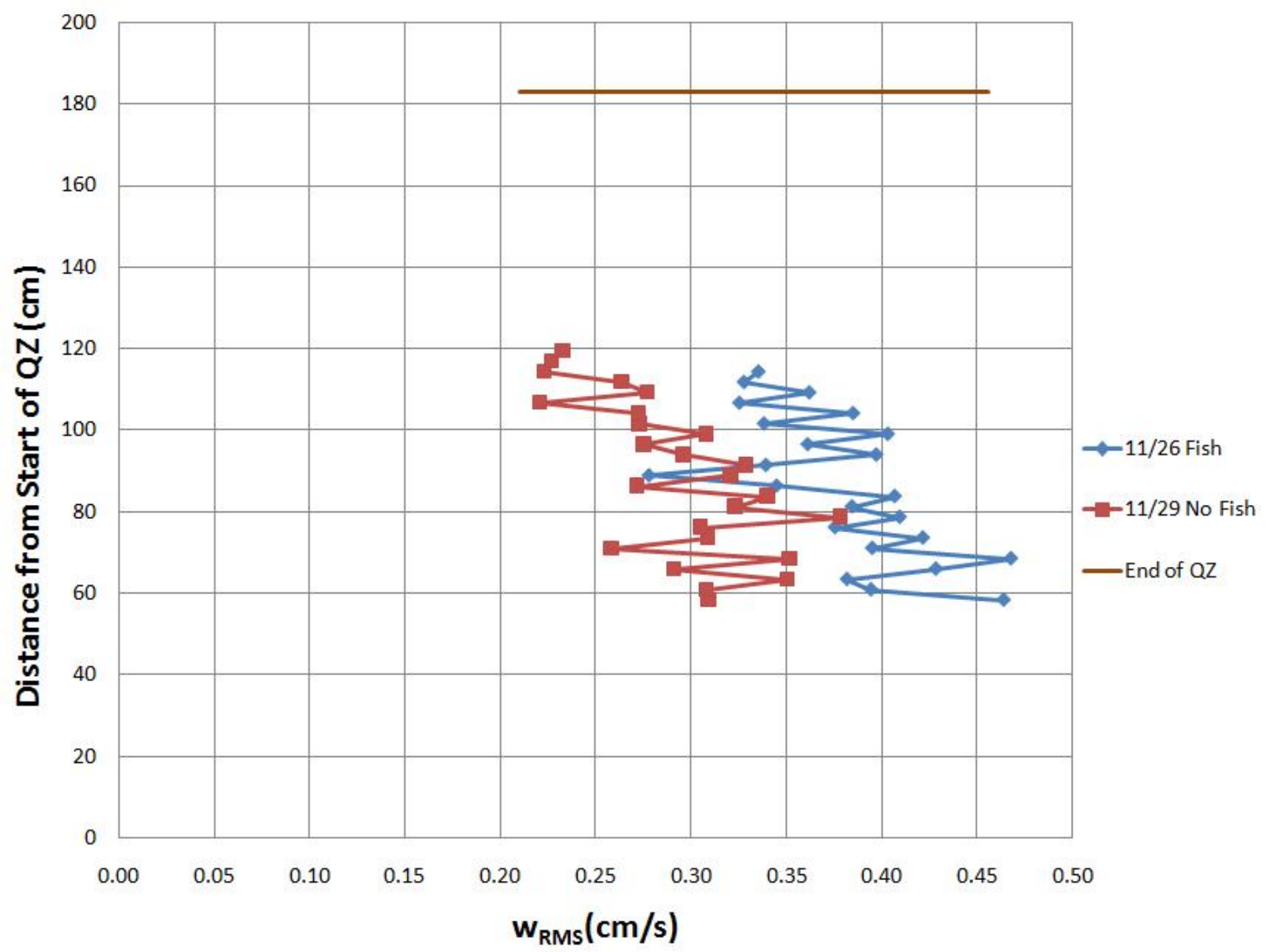

Figure 124: Streamwise Profiles of Root Mean Square Vertical Velocity 


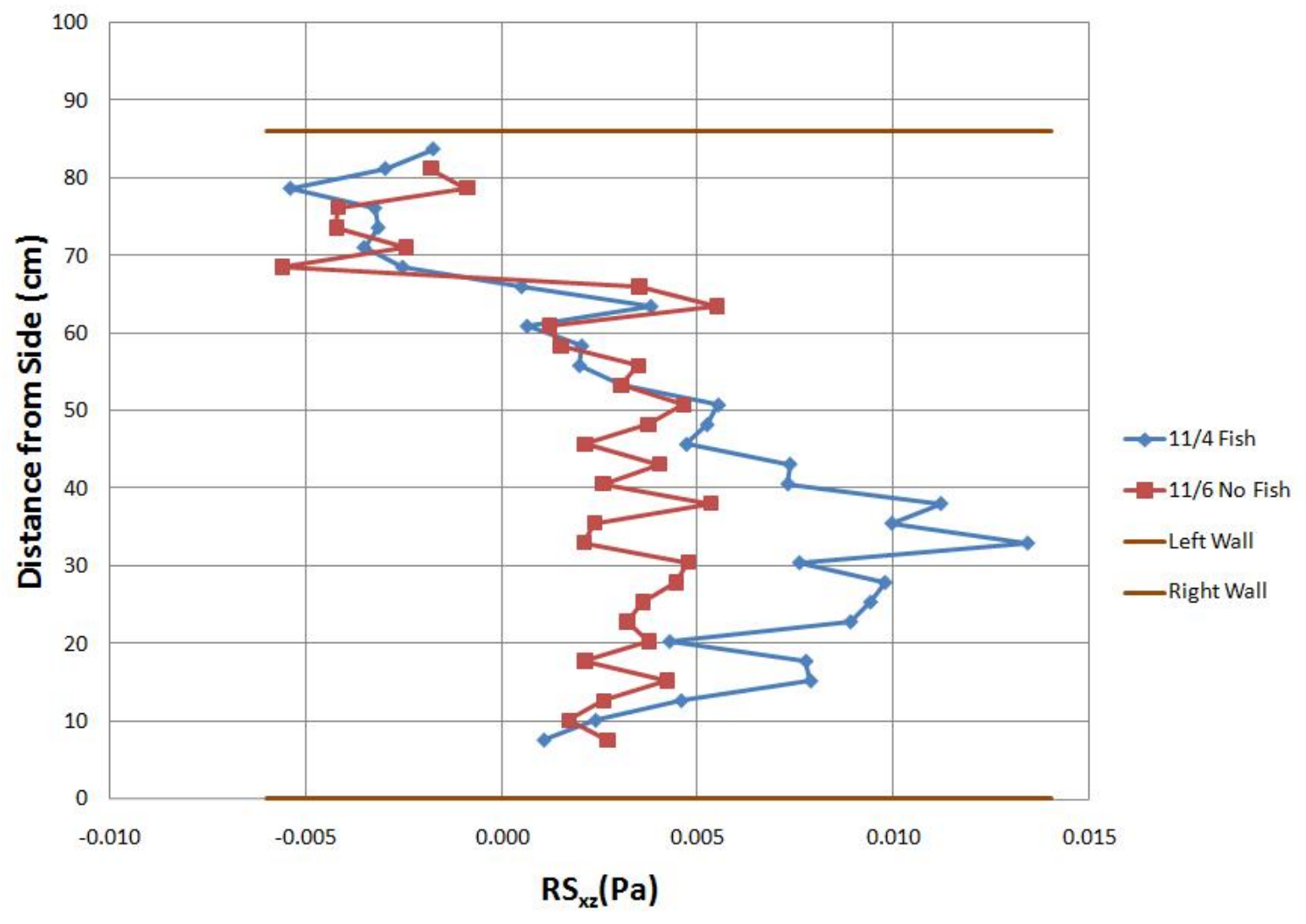

Figure 125: Transverse Profiles of $\mathrm{RS}_{\mathrm{xz}}$ 


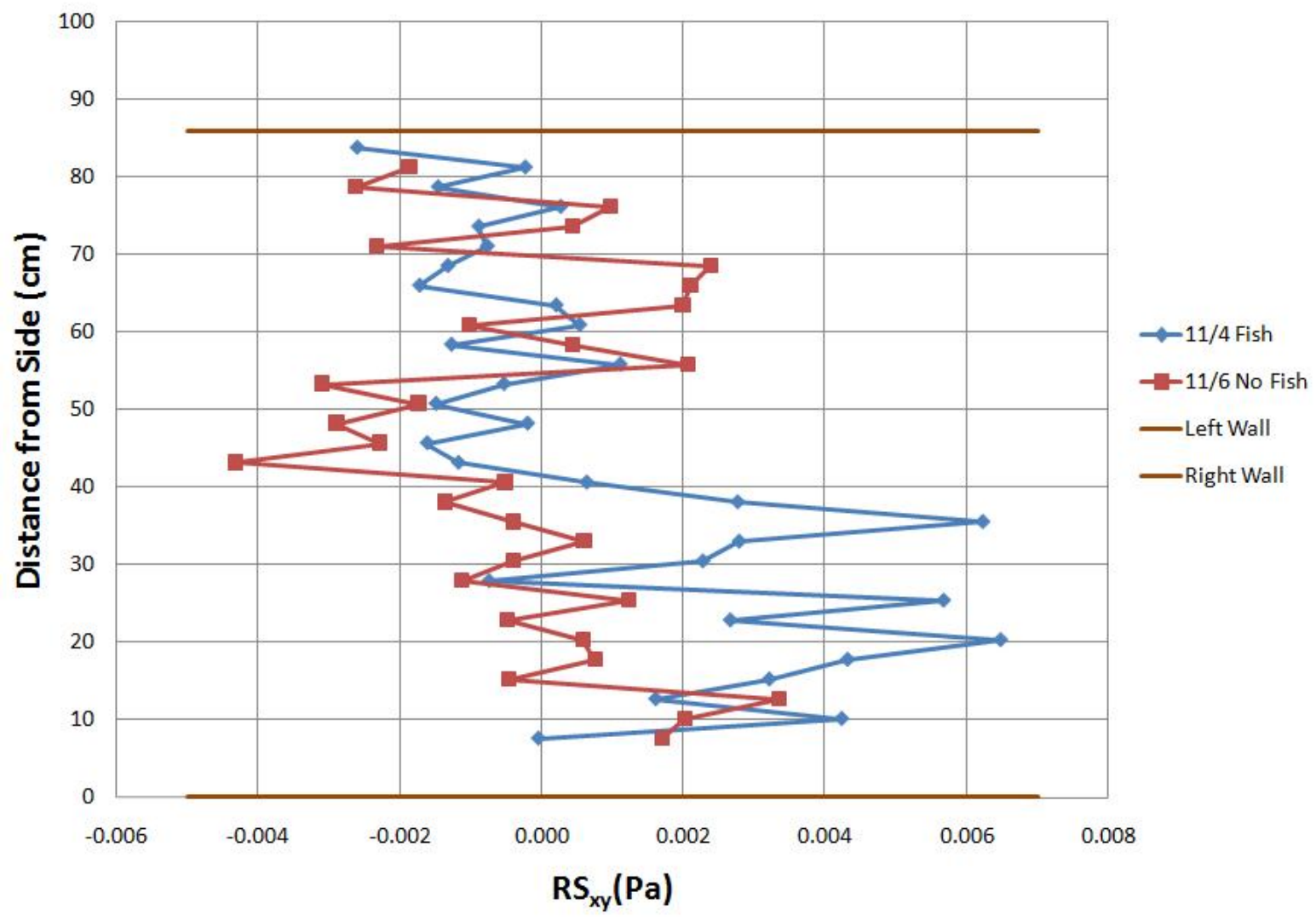

Figure 126: Transverse Profiles of $R_{x y}$ 


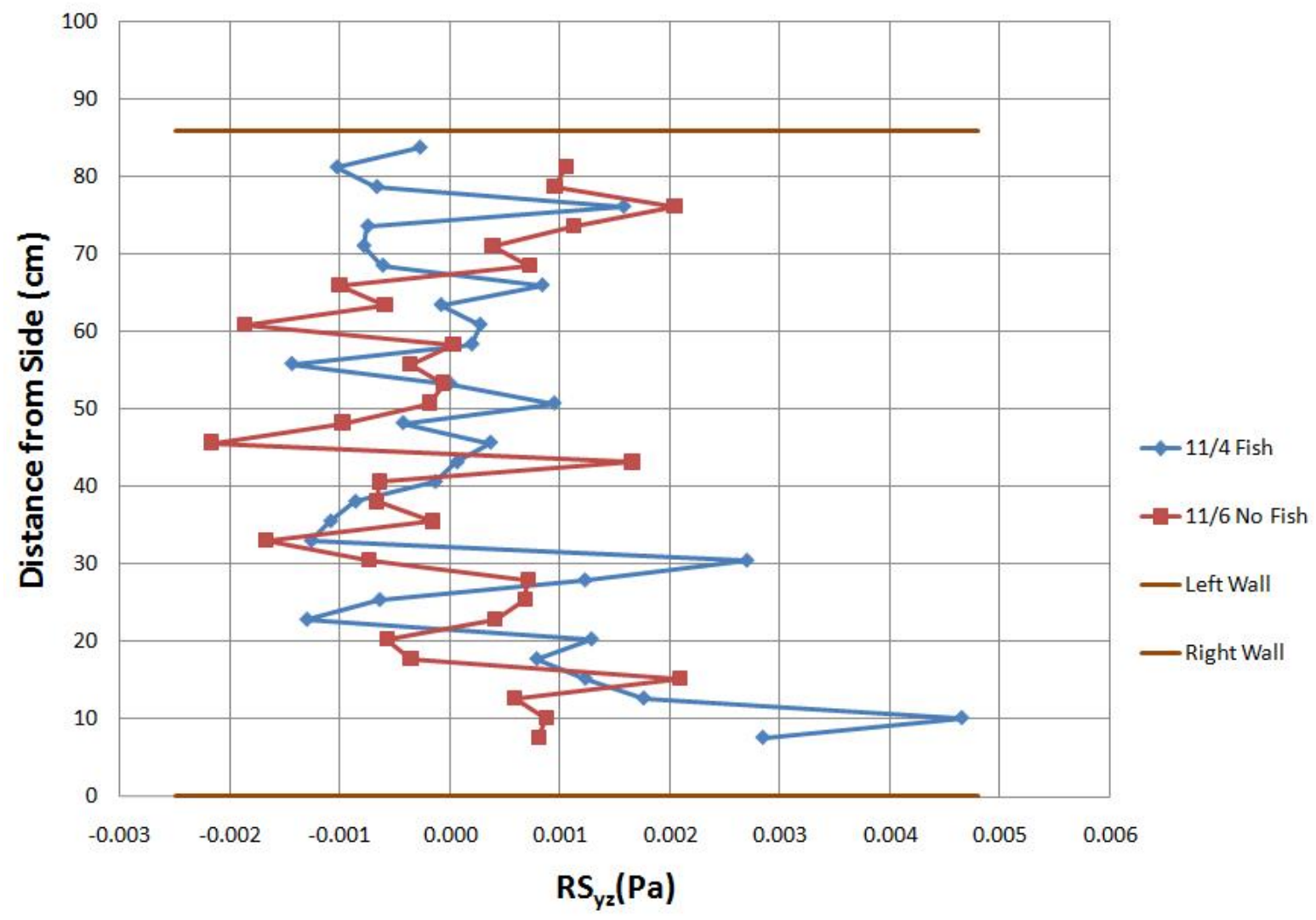

Figure 127: Transverse Profiles of $\mathrm{RS}_{\mathrm{yz}}$ 


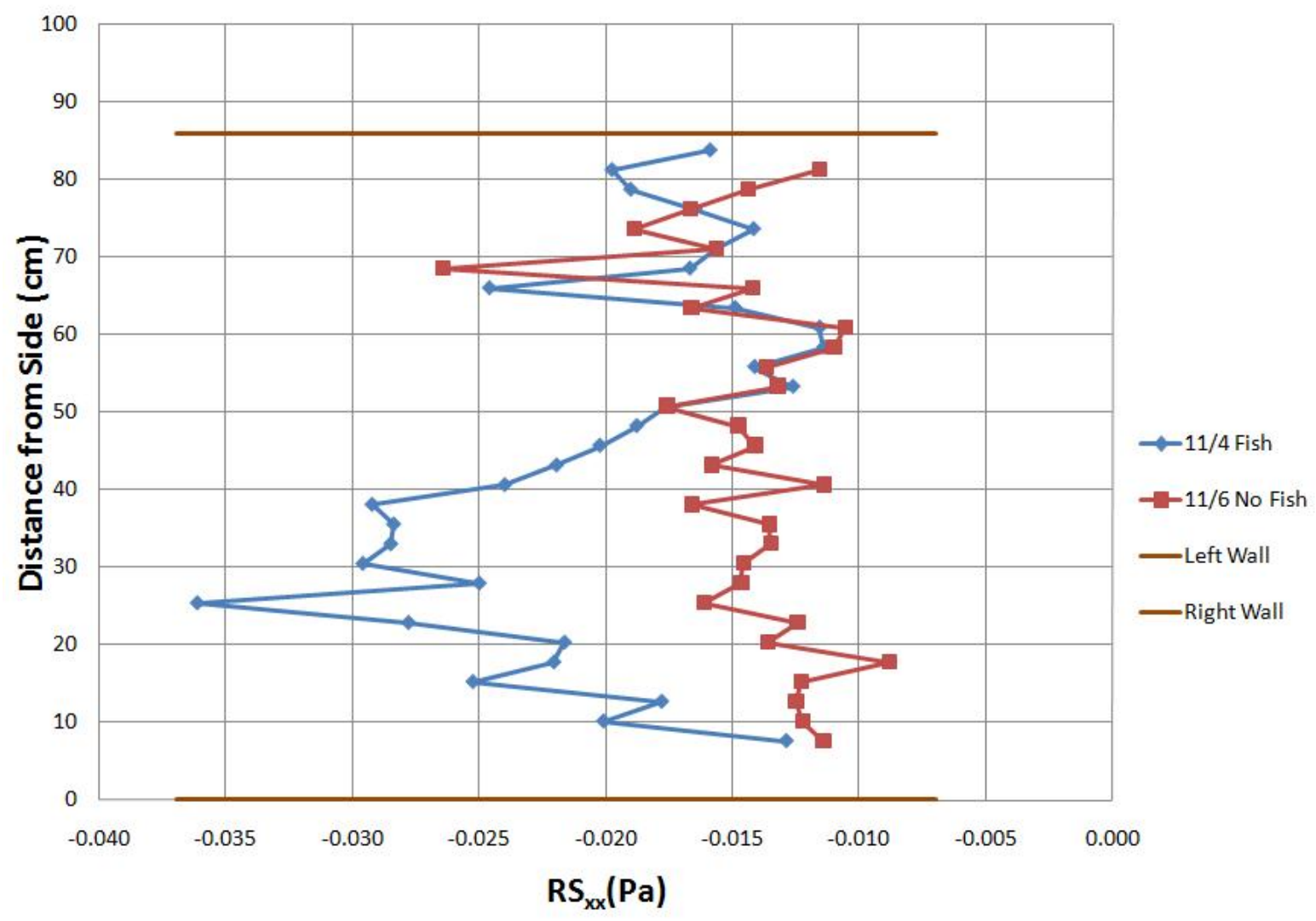

Figure 128: Transverse Profiles of $\mathrm{RS}_{\mathrm{xx}}$ 


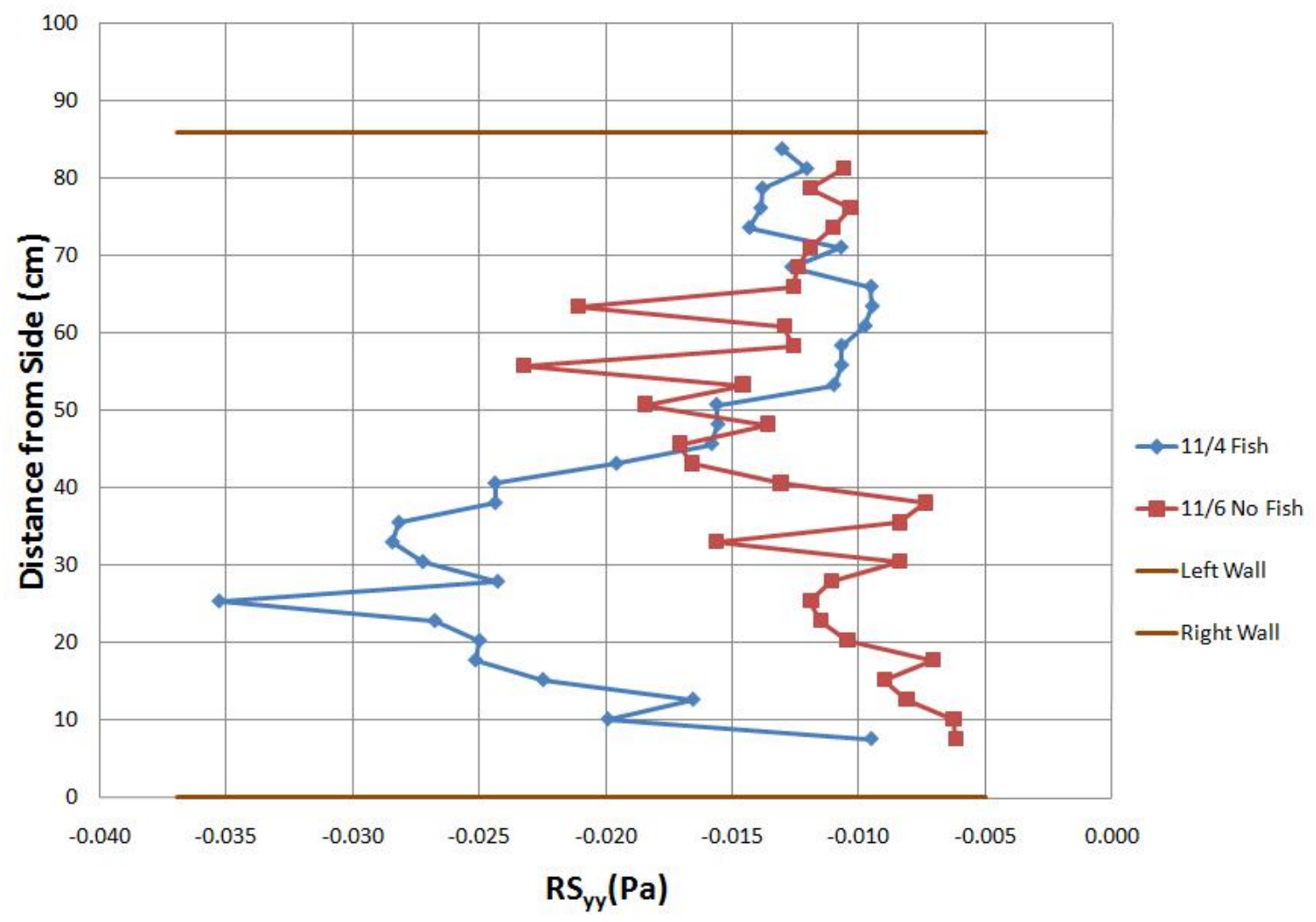

Figure 129: Transverse Profiles of $\mathrm{RS}_{\mathrm{yy}}$ 


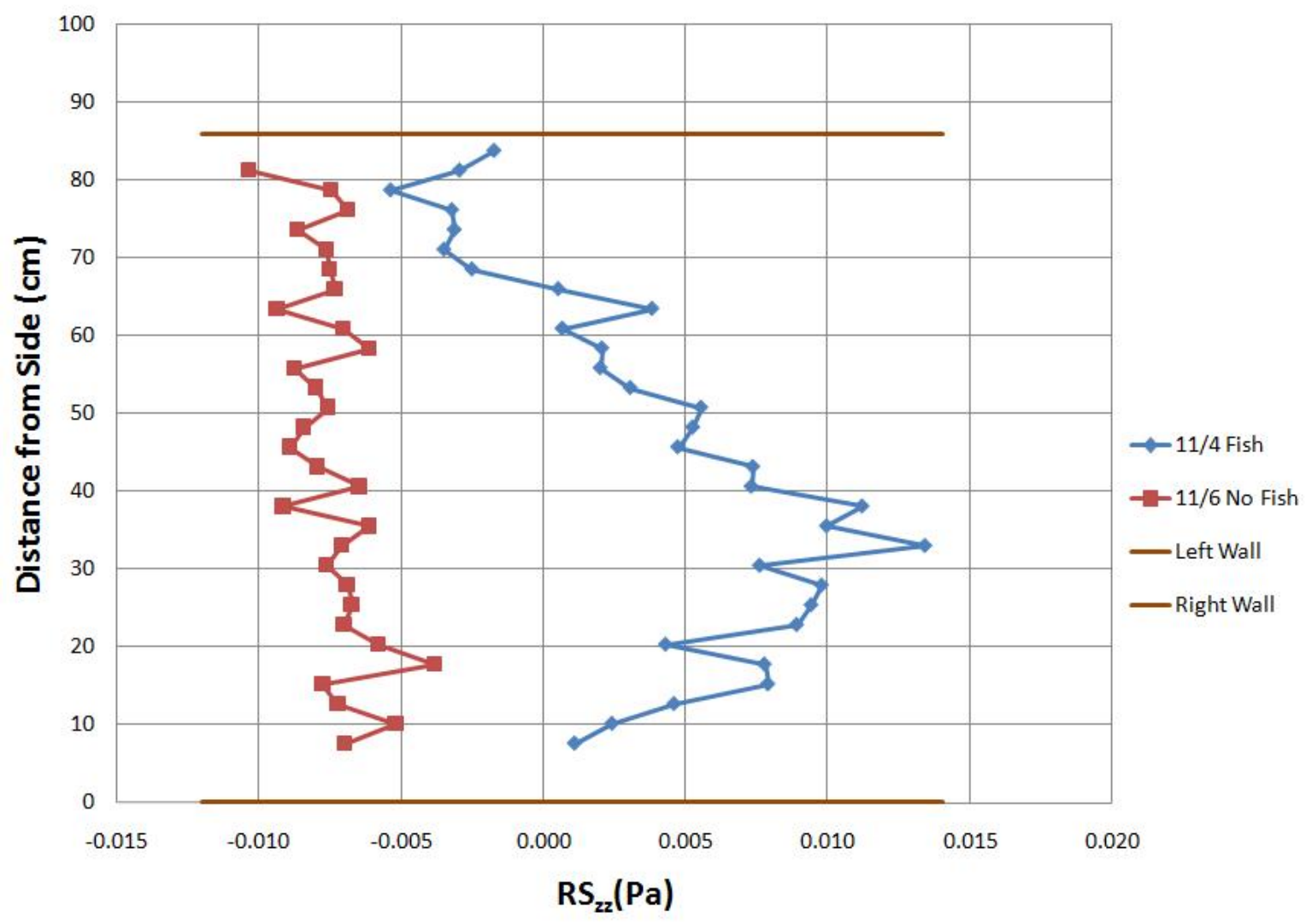

Figure 130: Transverse Profiles of $\mathbf{R S}_{\mathrm{zz}}$ 


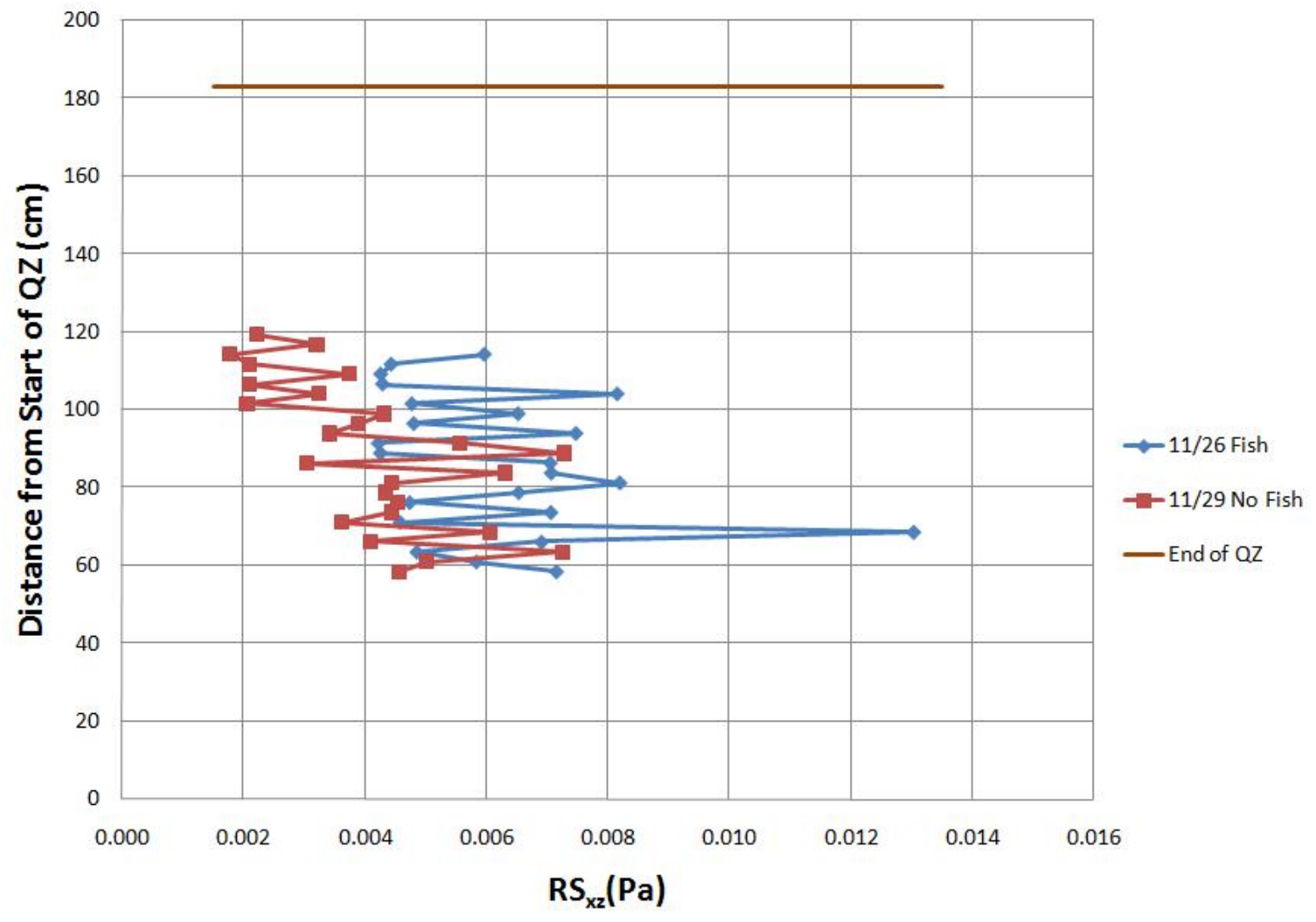

Figure 131: Streamwise Profiles of $\mathrm{RS}_{\mathrm{xz}}$ 


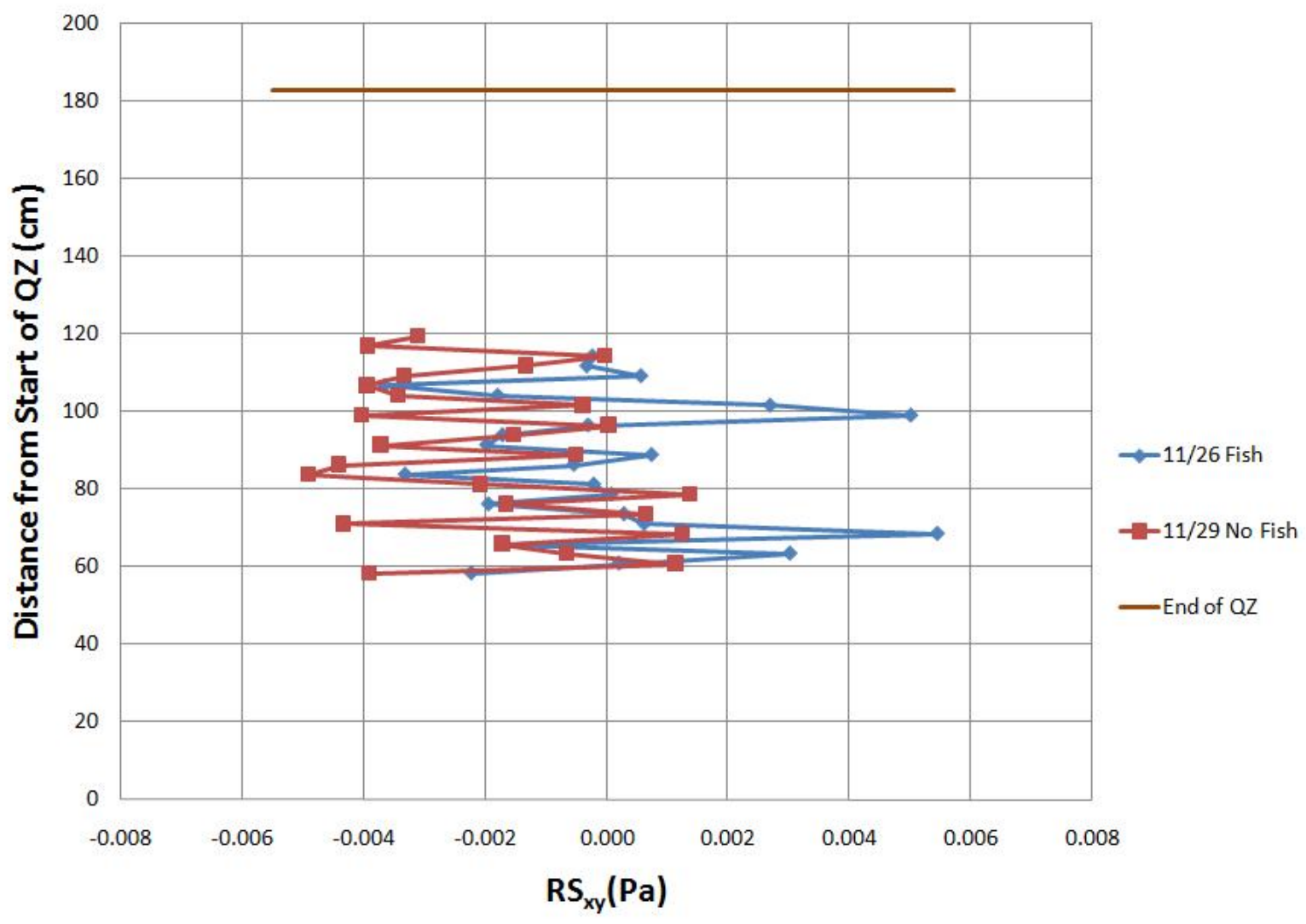

Figure 132: Streamwise Profiles of $\mathrm{RS}_{\mathrm{xy}}$ 


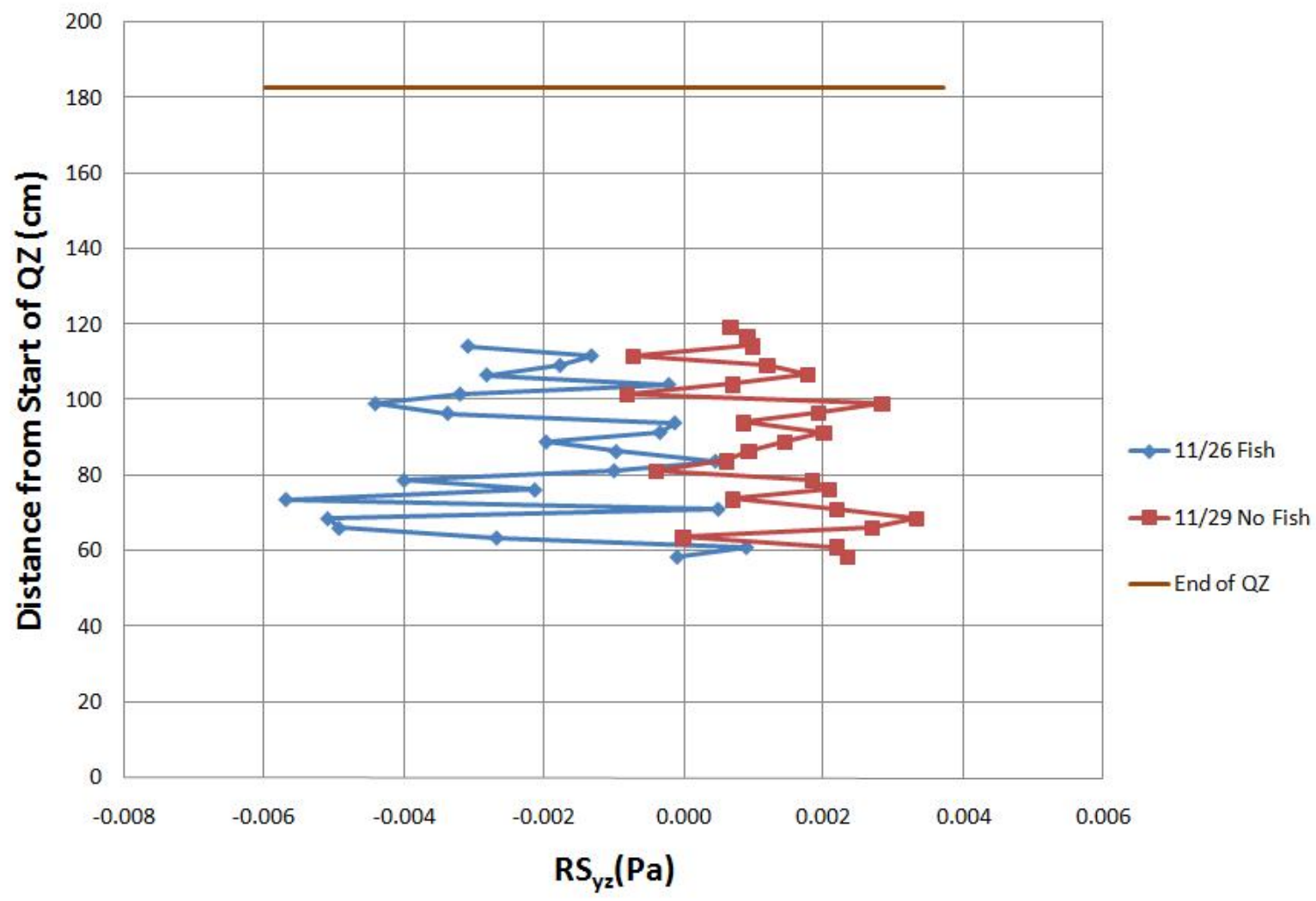

Figure 133: Streamwise Profiles of $\mathrm{RS}_{\mathbf{y z}}$ 


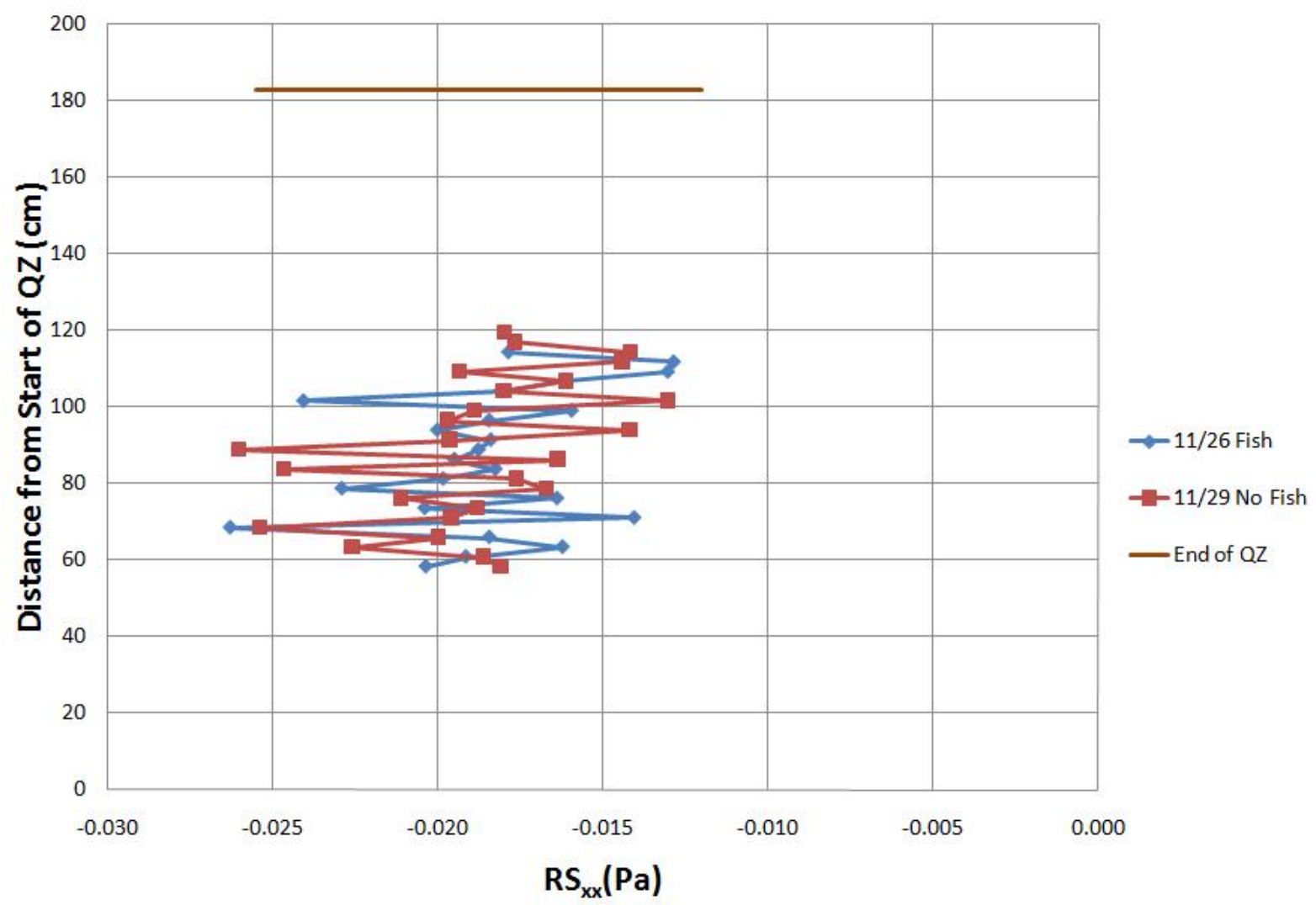

Figure 134: Streamwise Profiles of $R S_{x x}$ 


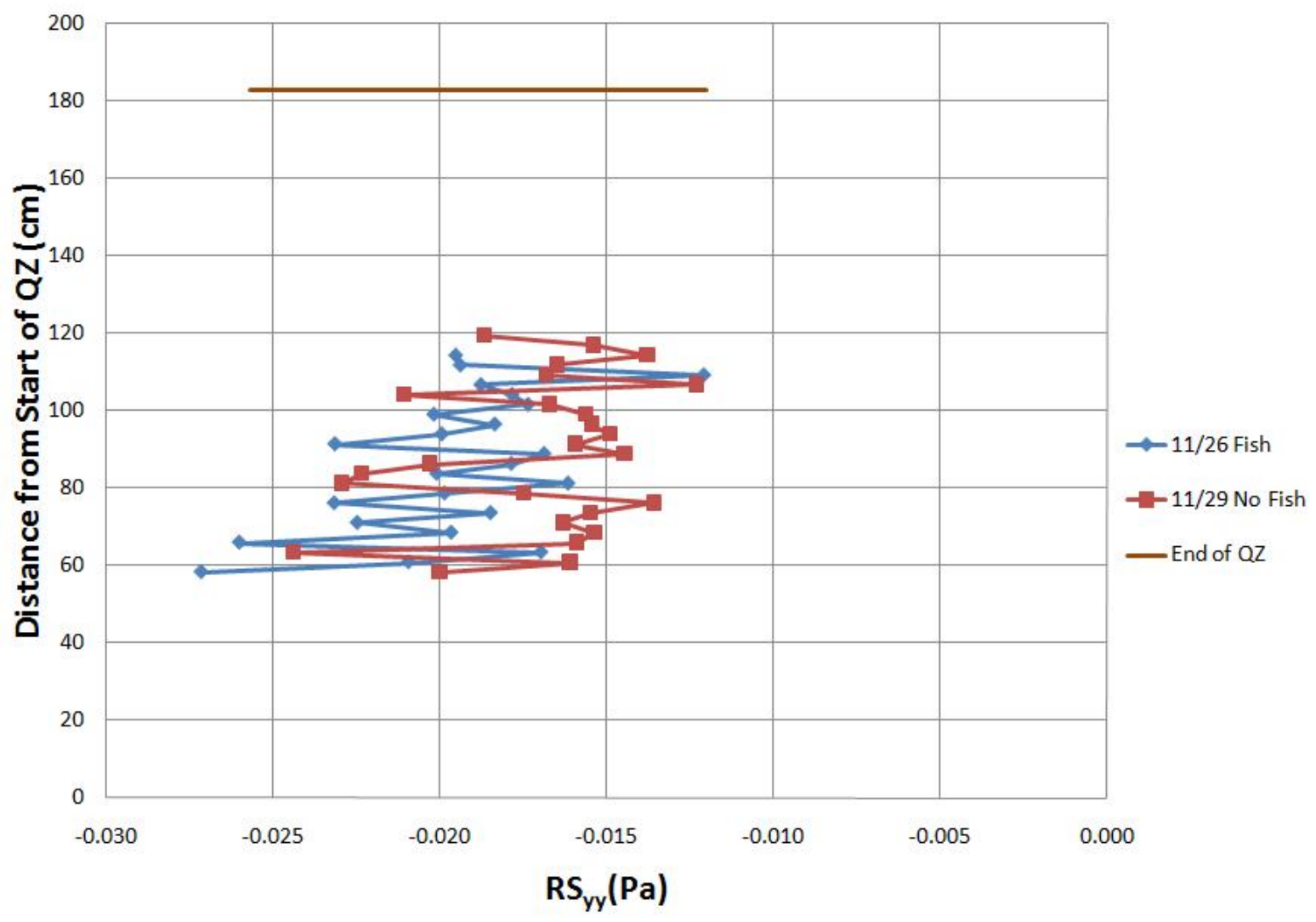

Figure 135: Streamwise Profiles of $\mathrm{RS}_{\mathbf{y y}}$ 


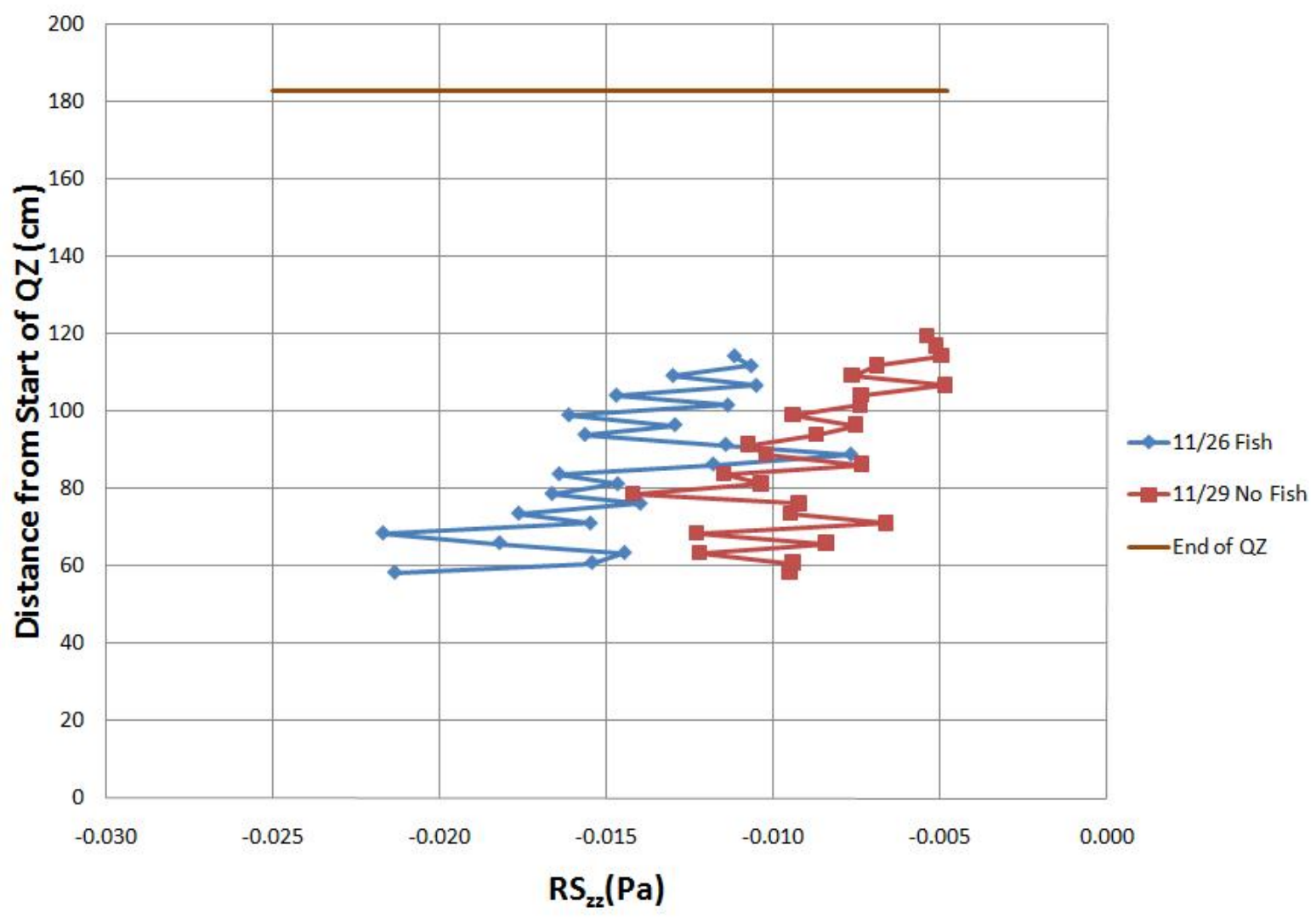

Figure 136: Streamwise Profiles of $\mathbf{R S}_{\mathrm{zz}}$

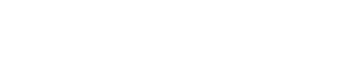

